NISTIR 8385

\title{
Cannabis Quality Assurance Program: Exercise 1 Final Report
}

Maryam Abdur-Rahman

Melissa M. Phillips

Walter B. Wilson

This publication is available free of charge from:

https://doi.org/10.6028/NIST.IR.8385

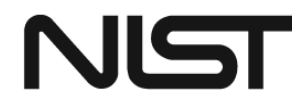

National Institute of Standards and Technology

U.S. Department of Commerce 
NISTIR 8385

\title{
Cannabis Quality Assurance Program: Exercise 1 Final Report
}

\author{
Maryam Abdur-Rahman \\ Melissa M. Phillips \\ Walter B. Wilson \\ Chemical Sciences Division \\ Material Measurement Laboratory
}

This publication is available free of charge from: https://doi.org/10.6028/NIST.IR.8385

July 2021

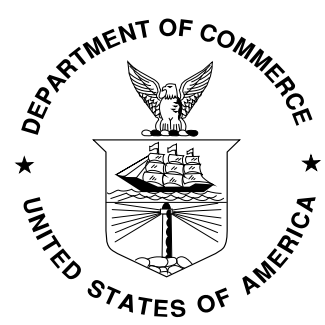

U.S. Department of Commerce Gina M. Raimondo, Secretary

National Institute of Standards and Technology James K. Olthoff, Performing the Non-Exclusive Functions and Duties of the Under Secretary of Commerce for Standards and Technology \& Director, National Institute of Standards and Technology 
Certain commercial entities, equipment, or materials may be identified in this document in order to describe an experimental procedure or concept adequately. Such identification is not intended to imply recommendation or endorsement by the National Institute of Standards and Technology, nor is it intended to imply that the entities, materials, or equipment are necessarily the best available for the purpose.

National Institute of Standards and Technology Interagency or Internal Report 8385

Natl. Inst. Stand. Technol. Interag. Intern. Rep. 8385, 196 pages (July 2021)

This publication is available free of charge from: https://doi.org/10.6028/NIST.IR.8385 


\section{TABLE OF CONTENTS}

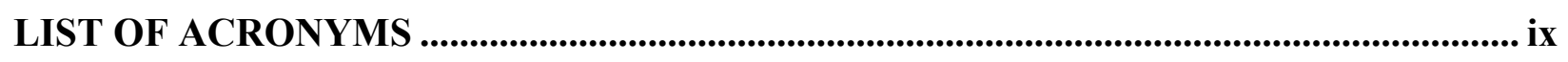

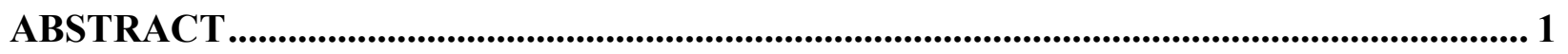

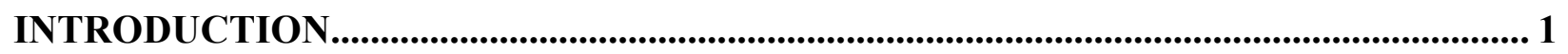

OVERVIEW OF DATA TREATMENT AND REPRESENTATION .................................. 2

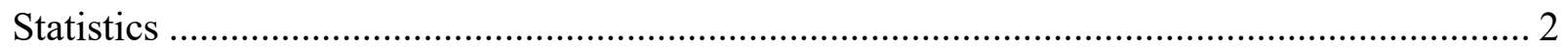

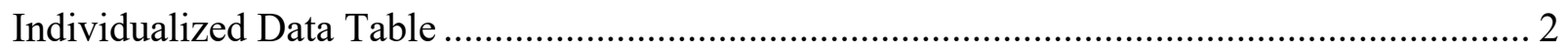

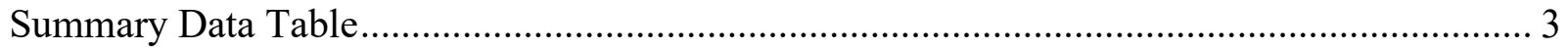

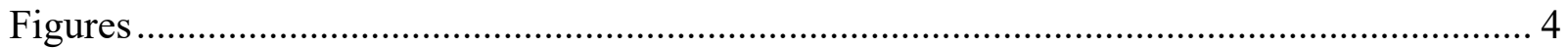

Data Summary View (Method Comparison Data Summary View) …................................... 4

Sample/Sample Comparison View ............................................................................. 4

SECTION 1: STUDY MATERIAL PREPARATION AND CHARACTERIZATION.......... 6

NIST Method for Study Material Characterization................................................................... 6

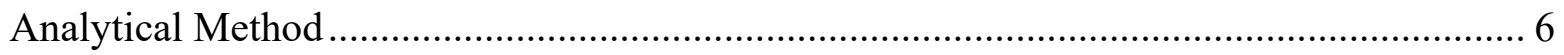

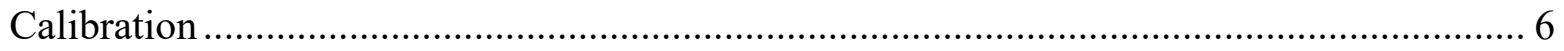

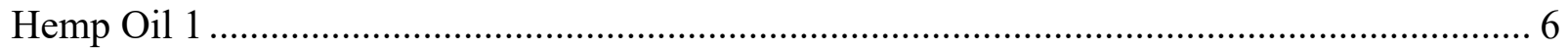

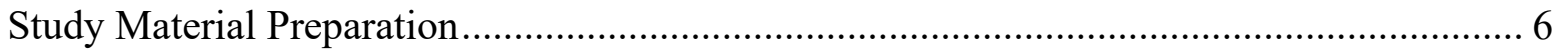

Study Material Characterization.................................................................................... 7

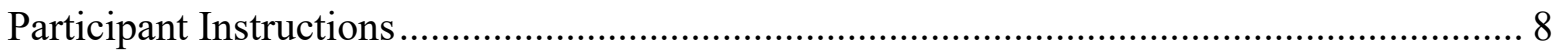

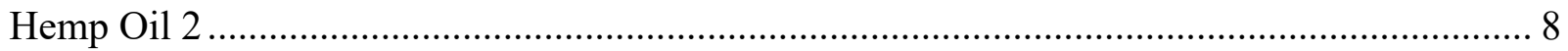

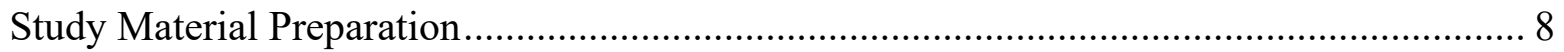

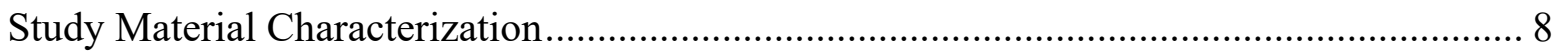

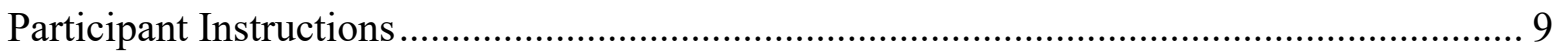

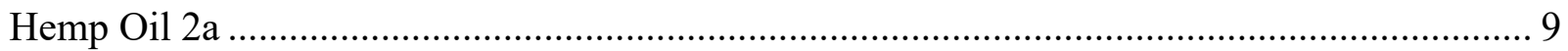

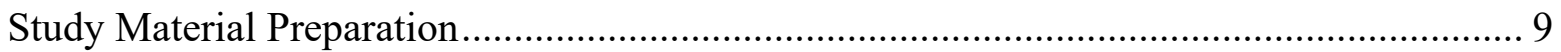

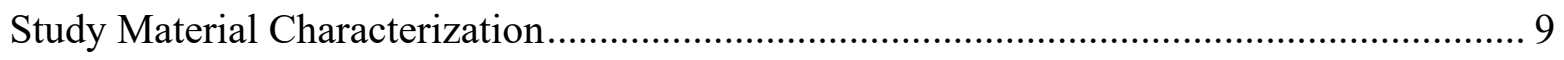

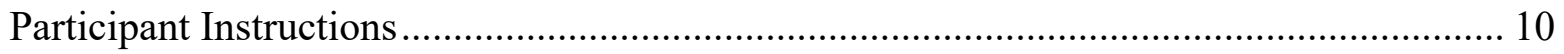

Figure 1-1. Extracted wavelength chromatogram for Hemp Oil 1 at $220 \mathrm{~nm}$......................... 11

Figure 1-2. UV absorbance spectra of the six suspected cannabinoid peaks in Hemp Oil 2 (black) and calibration standard (red).

Figure 1-3. (A) Selection of the extracted wavelength chromatogram at $220 \mathrm{~nm}$ for Hemp Oil 2. (B, C, D) UV absorbance spectra of suspected CBC peak in Hemp Oil 2 (black) at different chromatographic retention times compared to a calibration standard (red). 13

Figure 1-4. Extracted wavelength chromatogram at different wavelengths for the $\mathrm{CBC}$ chromatographic peak in Hemp Oil 2. 
Figure 1-5. Extracted wavelength chromatogram for Hemp Oil 2 at $220 \mathrm{~nm}$...................... 15

Figure 1-6. Extracted wavelength chromatogram for Hemp Oil 2a at $220 \mathrm{~nm}$.................... 16

SECTION 2: $\Delta^{9}$-THC, THCA, $\Delta^{8}$-THC, AND Total THC............................................... 17

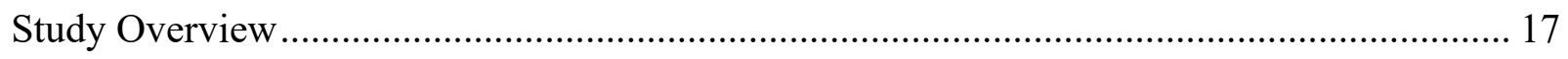

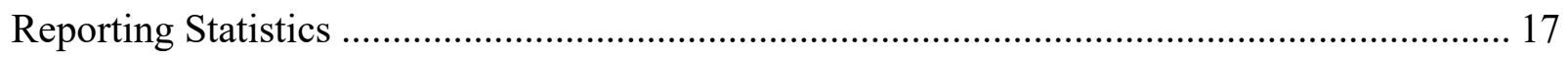

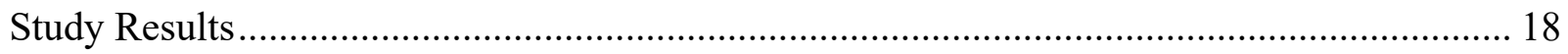

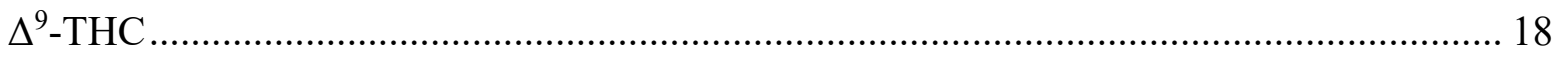

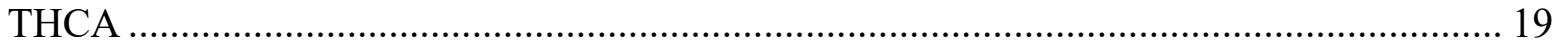

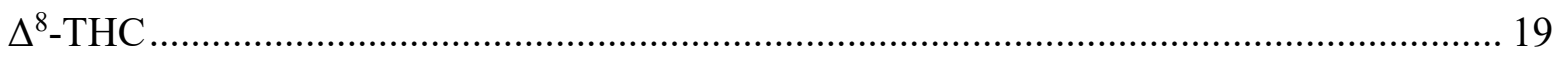

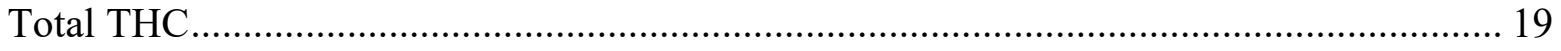

Study Discussion and Technical Recommendations ..................................................... 21

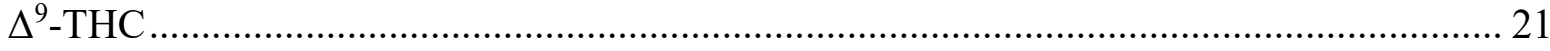

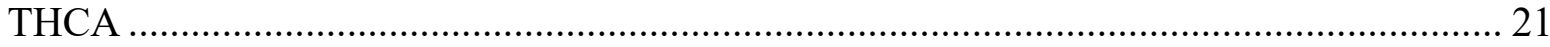

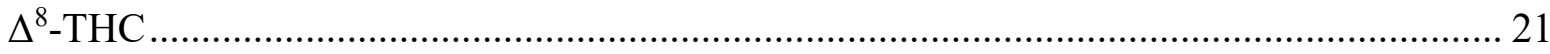

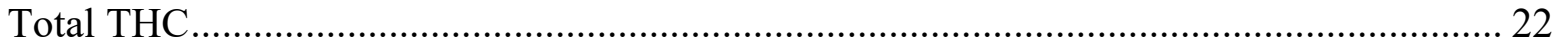

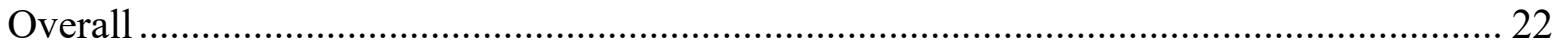

Table 2-1. Individualized data summary table (NIST) for $\Delta^{9}-\mathrm{THC}$, THCA, $\Delta^{8}-\mathrm{THC}$, and total

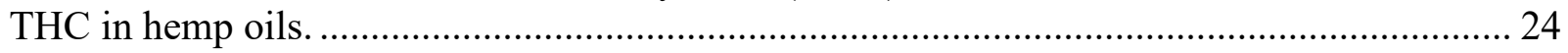

Table 2-2. Data summary table for $\Delta^{9}$-THC in hemp oils............................................. 25

Figure 2-1. $\Delta^{9}$-THC in Hemp Oil 1 (data summary view - analytical method)..................... 27

Figure 2-2. $\Delta^{9}$-THC in Hemp Oil 2 (data summary view - analytical method).................... 28

Figure 2-3. $\Delta^{9}$-THC in Hemp Oil 2a (data summary view - analytical method).................. 29

Figure 2-4. Laboratory means for $\Delta^{9}$-THC in Hemp Oil 1 and Hemp Oil 2 (sample/sample

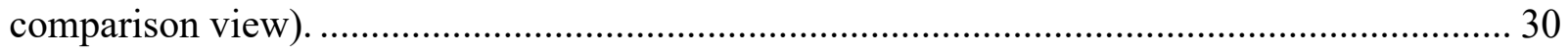

Table 2-3. Data summary table for THCA in hemp oils. .................................................. 31

Figure 2-5. THCA in Hemp Oil 1 (data summary view - analytical method)...................... 33

Figure 2-6. THCA in Hemp Oil 2 (data summary view - analytical method)....................... 34

Figure 2-7. THCA in Hemp Oil 2a (data summary view - analytical method).................... 35

Figure 2-8. Laboratory means for THCA in Hemp Oil 1 and Hemp Oil 2 (sample/sample

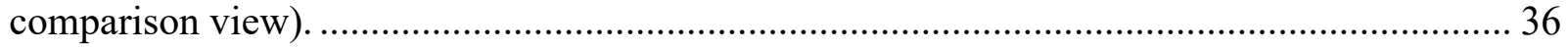

Table 2-4. Data summary table for $\Delta^{8}$-THC in hemp oils............................................. 37

Figure 2-9. $\Delta^{8}$-THC in Hemp Oil 1 (data summary view - analytical method). ................... 39

Figure 2-10. $\Delta^{8}$-THC in Hemp Oil 2 (data summary view - analytical method). ................. 40

Figure 2-11. $\Delta^{8}$-THC in Hemp Oil 2a (data summary view - analytical method)................ 41 
Figure 2-12. Laboratory means for $\Delta^{8}$-THC in Hemp Oil 1 and Hemp Oil 2 (sample/sample comparison view).

Table 2-5. Data summary table for total THC in hemp oils........................................... 43

Figure 2-13. Total THC in Hemp Oil 1 (data summary view - analytical method).............. 45

Figure 2-14. Total THC in Hemp Oil 2 (data summary view - analytical method).............. 46

Figure 2-15. Total THC in Hemp Oil 2a (data summary view - analytical method).............. 47

Figure 2-16. Laboratory means for total THC in Hemp Oil 1 and Hemp Oil 2 (sample/sample

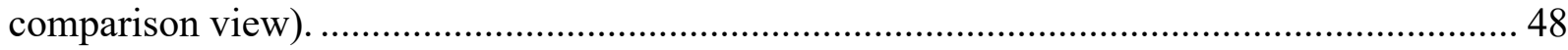

SECTION 3: CBD, CBDA, and Total CBD ............................................................................ 49

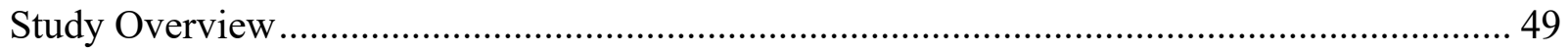

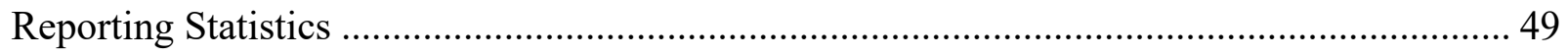

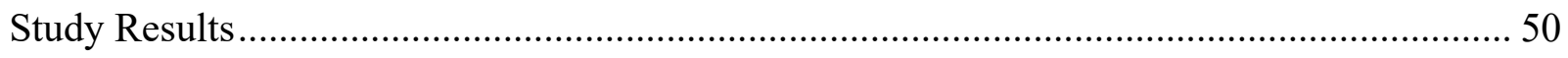

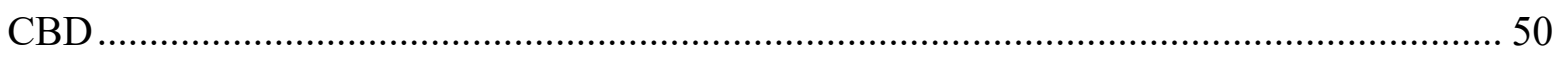

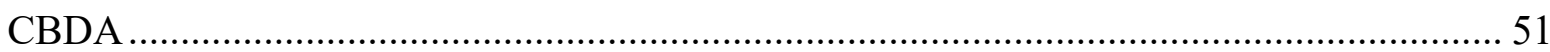

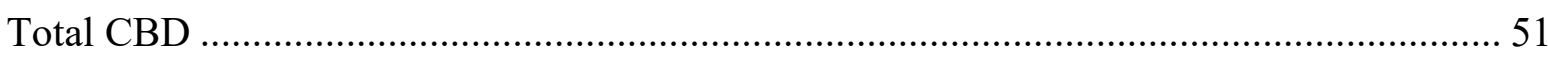

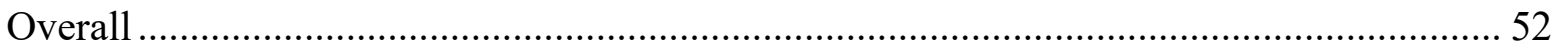

Study Discussion and Technical Recommendations.................................................... 52

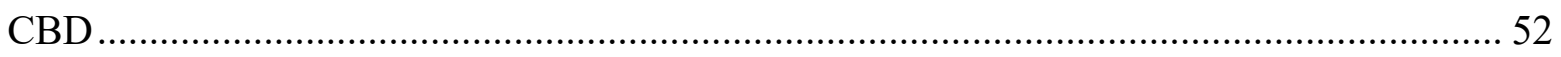

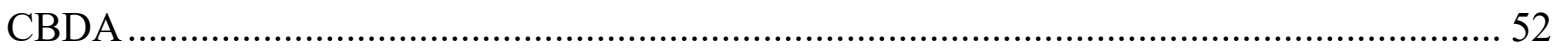

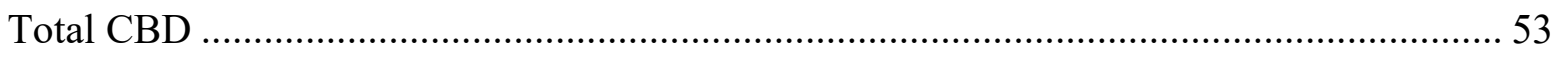

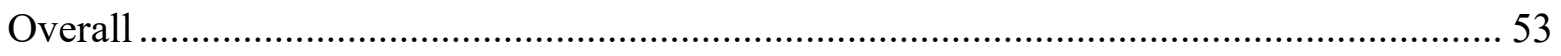

Table 3-1. Individualized data summary table (NIST) for CBD, CBDA, and total CBD in hemp

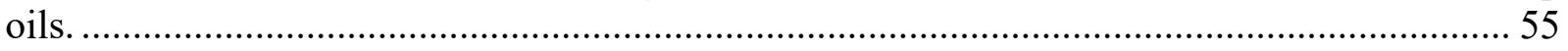

Table 3-2. Data summary table for CBD in hemp oils..................................................... 56

Figure 3-1. CBD in Hemp Oil 1 (data summary view - analytical method). ....................... 58

Figure 3-2. CBD in Hemp Oil 2 (data summary view - analytical method). ....................... 59

Figure 3-3. CBD in Hemp Oil 2a (data summary view - analytical method)....................... 60

Figure 3-4. Laboratory means for CBD in Hemp Oil 1 and Hemp Oil 2 (sample/sample

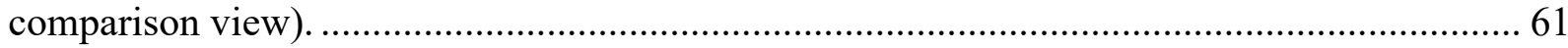

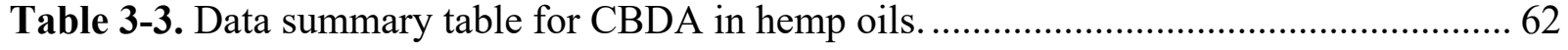

Figure 3-5. CBDA in Hemp Oil 1 (data summary view - analytical method)..................... 64

Figure 3-6. CBDA in Hemp Oil 2 (data summary view - analytical method)...................... 65

Figure 3-7. CBDA in Hemp Oil 2a (data summary view - analytical method).................... 66

Figure 3-8. Laboratory means for CBDA in Hemp Oil 1 and Hemp Oil 2 (sample/sample

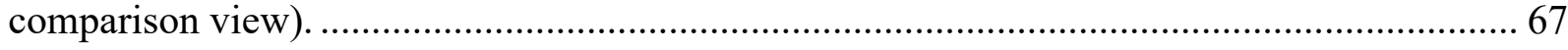

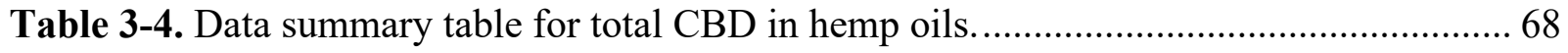


Figure 3-9. Total CBD in Hemp Oil 1 (data summary view - analytical method)................ 70

Figure 3-10. Total CBD in Hemp Oil 2 (data summary view - analytical method).............. 71

Figure 3-11. Total CBD in Hemp Oil 2a (data summary view - analytical method)............ 72

Figure 3-12. Laboratory means for total CBD in Hemp Oil 1 and Hemp Oil 2 (sample/sample

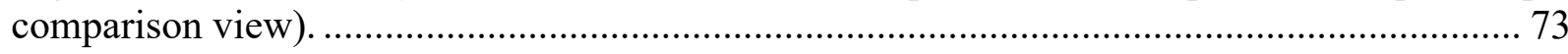

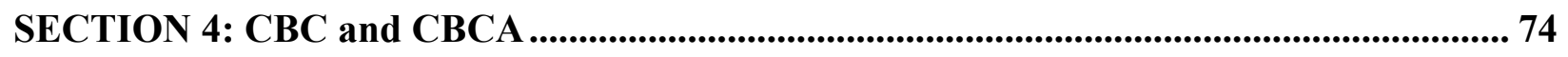

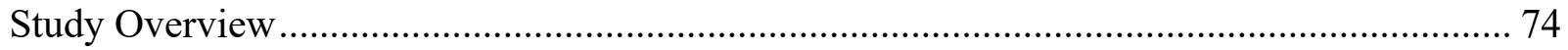

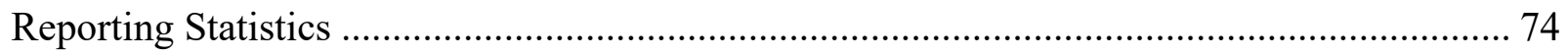

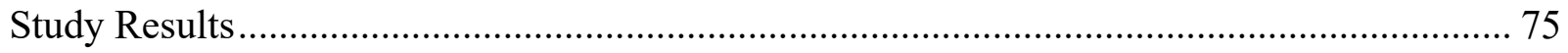

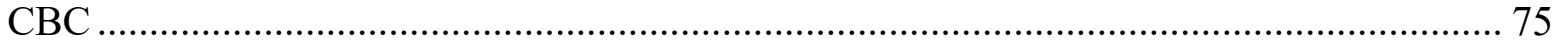

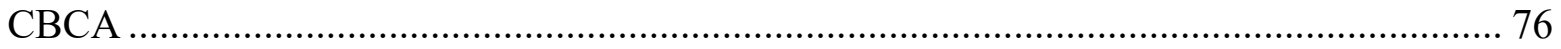

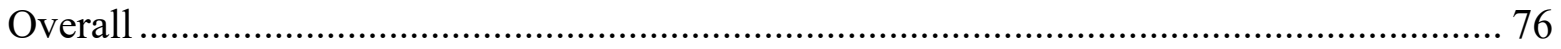

Study Discussion and Technical Recommendations.................................................. 76

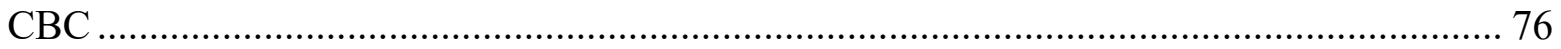

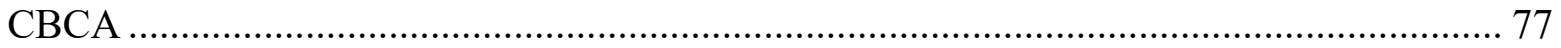

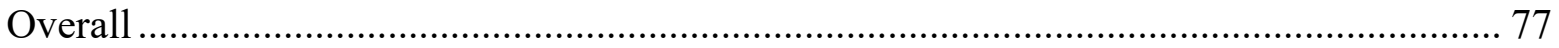

Table 4-1. Individualized data summary table (NIST) for CBC and CBCA in hemp oils...... 79

Table 4-2. Data summary table for CBC in hemp oils.................................................. 80

Figure 4-1. CBC in Hemp Oil 1 (data summary view - analytical method)........................ 82

Figure 4-2. CBC in Hemp Oil 2 (data summary view - analytical method)........................ 83

Figure 4-3. CBC in Hemp Oil 2a (data summary view - analytical method)...................... 84

Figure 4-4. Laboratory means for $\mathrm{CBC}$ in Hemp Oil 1 and Hemp Oil 2 (sample/sample

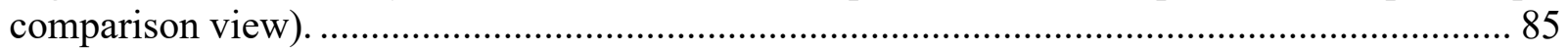

Table 4-3. Data summary table for CBCA in hemp oils. ................................................. 86

Figure 4-5. CBCA in Hemp Oil 1 (data summary view - analytical method)...................... 87

Figure 4-6. CBCA in Hemp Oil 2 (data summary view - analytical method)...................... 88

Figure 4-7. CBCA in Hemp Oil 2a (data summary view - analytical method).................... 89

Figure 4-8. Laboratory means for CBCA in Hemp Oil 1 and Hemp Oil 2 (sample/sample

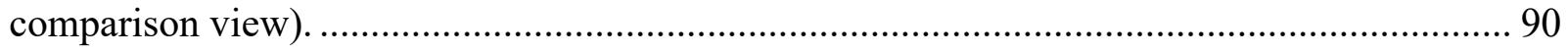

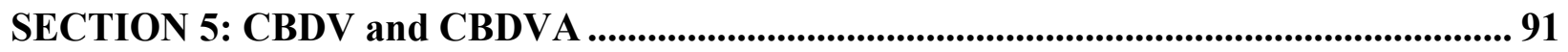

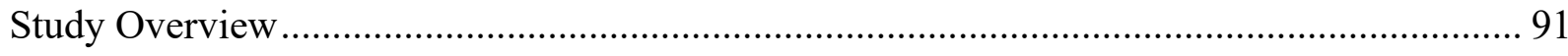

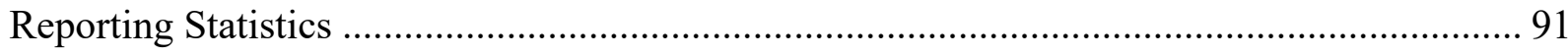

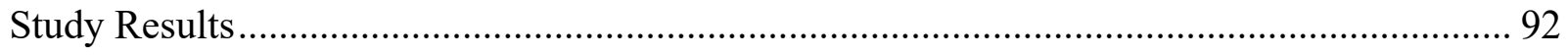

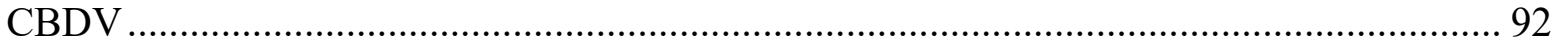

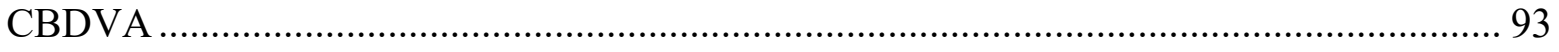




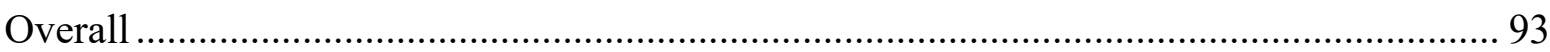

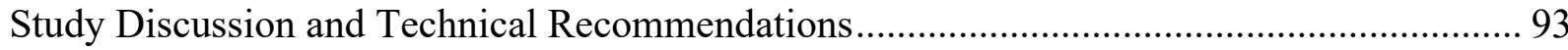

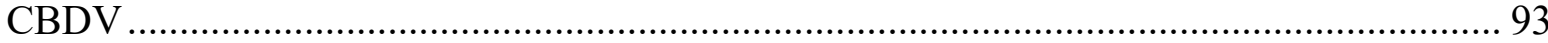

CBDVA …

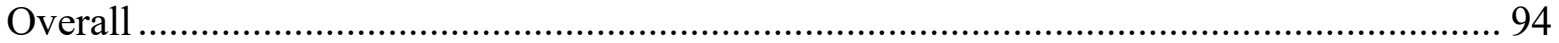

Table 5-1. Individualized data summary table (NIST) for CBDV and CBDVA in hemp oils. 95

Table 5-2. Data summary table for CBDV in hemp oils..................................................... 96

Figure 5-1. CBDV in Hemp Oil 1 (data summary view - analytical method)......................... 98

Figure 5-2. CBDV in Hemp Oil 2 (data summary view - analytical method)........................ 99

Figure 5-3. CBDV in Hemp Oil 2a (data summary view - analytical method).................... 100

Figure 5-4. Laboratory means for CBDV in Hemp Oil 1 and Hemp Oil 2 (sample/sample

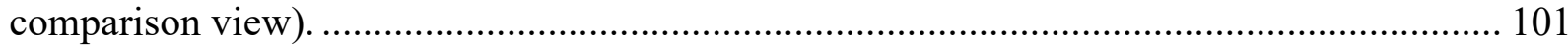

Table 5-3. Data summary table for CBDVA in hemp oils.................................................. 102

Figure 5-5. CBDVA in Hemp Oil 1 (data summary view - analytical method).................... 103

Figure 5-6. CBDVA in Hemp Oil 2 (data summary view - analytical method).................... 104

Figure 5-7. CBDVA in Hemp Oil 2a (data summary view - analytical method)................... 105

Figure 5-8. Laboratory means for CBDVA in Hemp Oil 1 and Hemp Oil 2 (sample/sample

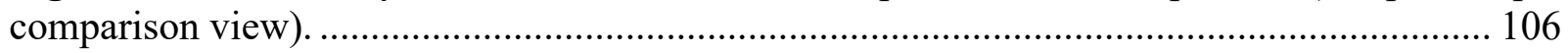

SECTION 6: CBG and CBGA.................................................................................................... 107

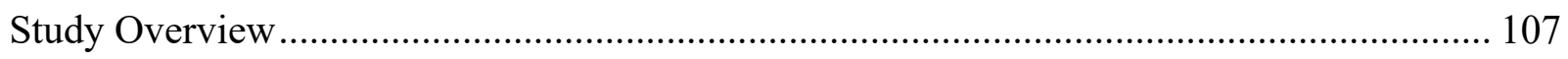

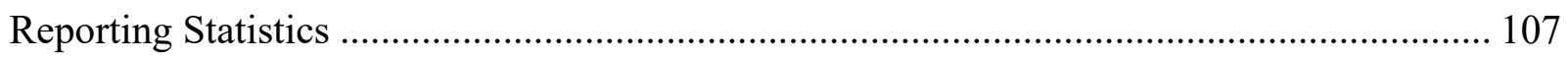

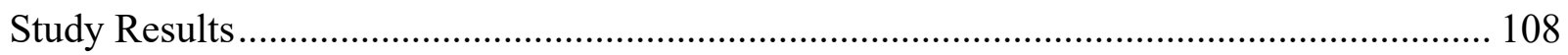

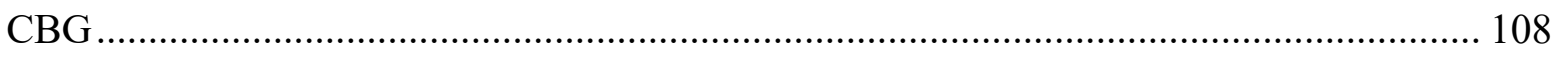

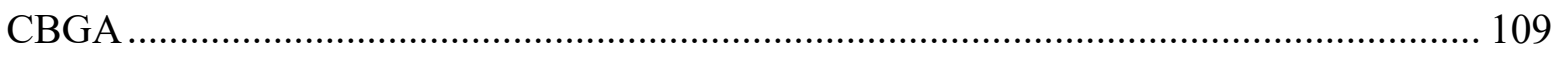

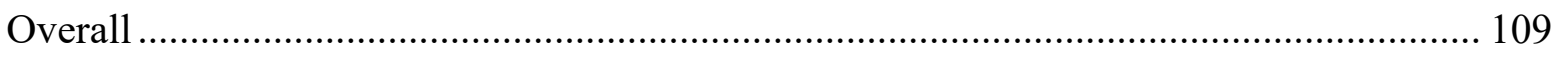

Study Discussion and Technical Recommendations........................................................... 109

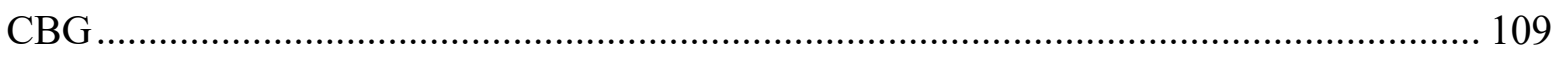

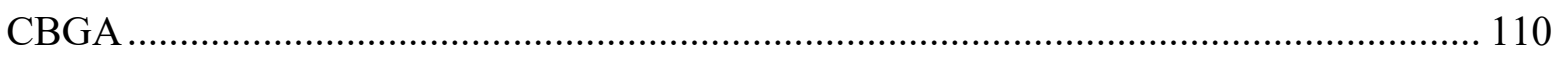

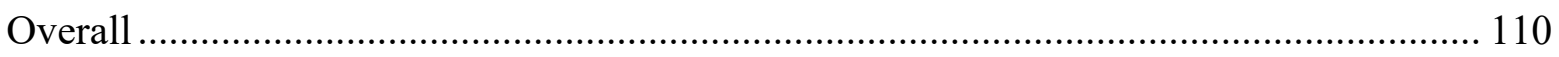

Table 6-1. Individualized data summary table (NIST) for CBG and CBGA in hemp oils. ... 112

Table 6-2. Data summary table for CBG in hemp oils...................................................... 113

Figure 6-1. CBG in Hemp Oil 1 (data summary view - analytical method). ........................ 115

Figure 6-2. CBG in Hemp Oil 2 (data summary view - analytical method). ........................ 116

Figure 6-3. CBG in Hemp Oil 2a (data summary view - analytical method)....................... 117 
Figure 6-4. Laboratory means for $\mathrm{CBG}$ in Hemp Oil 1 and Hemp Oil 2 (sample/sample comparison view).

Table 6-3. Data summary table for CBA in hemp oils. 119

Figure 6-5. CBGA in Hemp Oil 1 (data summary view - analytical method)....... 121

Figure 6-6. CBGA in Hemp Oil 2 (data summary view - analytical method)....... 122

Figure 6-7. CBGA in Hemp Oil 2a (data summary view - analytical method). 123

Figure 6-8. Laboratory means for CBGA in Hemp Oil 1 and Hemp Oil 2 (sample/sample comparison view).

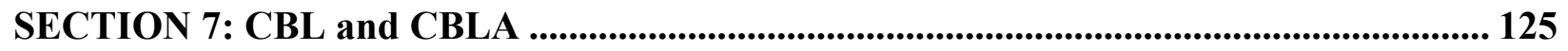

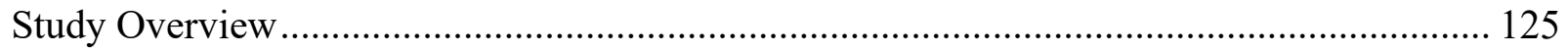

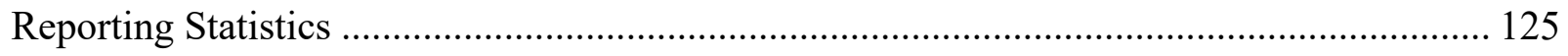

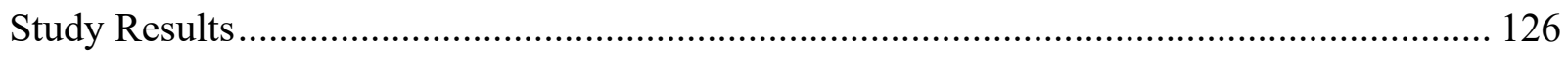

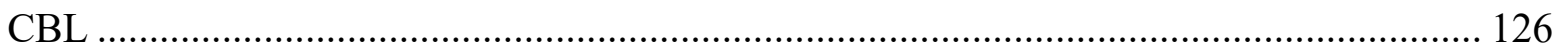

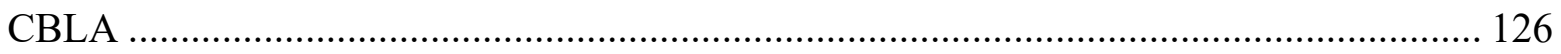

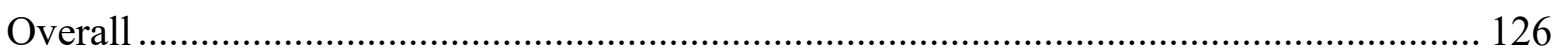

Study Discussion and Technical Recommendations................................................... 127

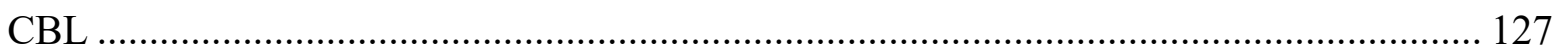

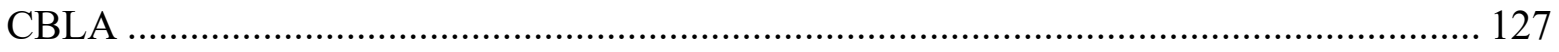

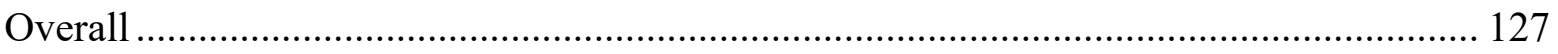

Table 7-1. Individualized data summary table (NIST) for CBL and CBLA in hemp oils. .... 129

Table 7-2. Data summary table for CBL in hemp oils. ................................................. 130

Figure 7-1. CBL in Hemp Oil 1 (data summary view - analytical method)........................ 131

Figure 7-2. CBL in Hemp Oil 2 (data summary view - analytical method)....................... 132

Figure 7-3. CBL in Hemp Oil 2a (data summary view - analytical method)..................... 133

Figure 7-4. Laboratory means for CBL in Hemp Oil 1 and Hemp Oil 2 (sample/sample comparison view)...................................................................................... 134

Table 7-3. Data summary table for CBLA in hemp oils. ........................................... 135

Figure 7-5. CBLA in Hemp Oil 1 (data summary view - analytical method).................... 136

Figure 7-6. CBLA in Hemp Oil 2 (data summary view - analytical method).................... 137

Figure 7-7. CBLA in Hemp Oil 2a (data summary view - analytical method). ................. 138

Figure 7-8. Laboratory means for CBLA in Hemp Oil 1 and Hemp Oil 2 (sample/sample

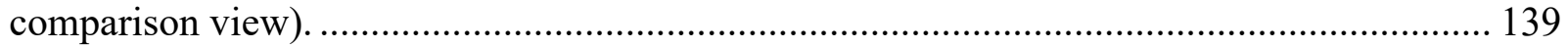

SECTION 8: CBN and CBNA ............................................................................................ 140

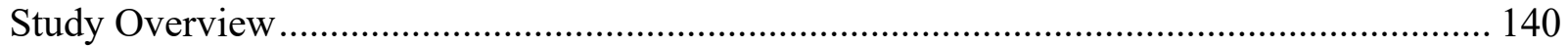

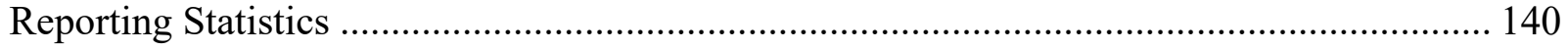




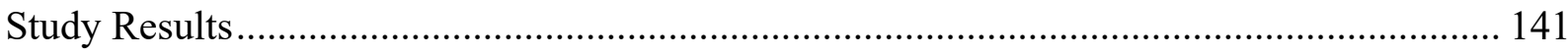

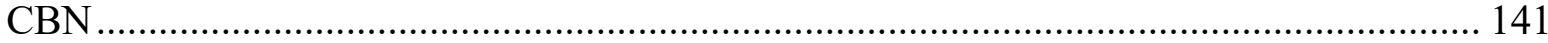

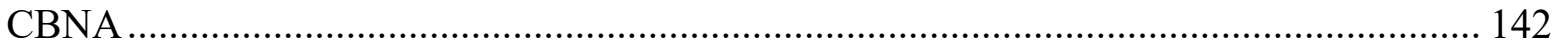

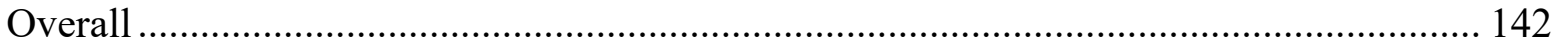

Study Discussion and Technical Recommendations............................................................ 142

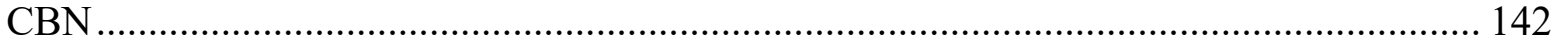

CBNA …

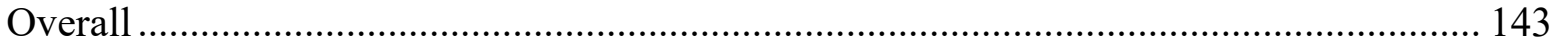

Table 8-1. Individualized data summary table (NIST) for CBN and CBNA in hemp oils... 145

Table 8-2. Data summary table for $\mathrm{CBN}$ in hemp oils.................................................... 146

Figure 8-1. CBN in Hemp Oil 1 (data summary view - analytical method). ........................ 148

Figure 8-2. CBN in Hemp Oil 2 (data summary view - analytical method). ....................... 149

Figure 8-3. CBN in Hemp Oil 2a (data summary view - analytical method)........................ 150

Figure 8-4. Laboratory means for CBN in Hemp Oil 1 and Hemp Oil 2 (sample/sample

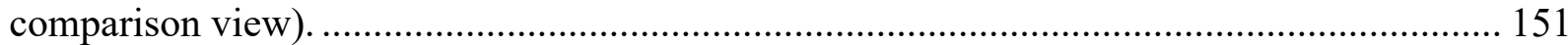

Table 8-3. Data summary table for CBNA in hemp oils.................................................. 152

Figure 8-5. CBNA in Hemp Oil 1 (data summary view - analytical method)....................... 153

Figure 8-6. CBNA in Hemp Oil 2 (data summary view - analytical method)....................... 154

Figure 8-7. CBNA in Hemp Oil 2a (data summary view - analytical method)..................... 155

Figure 8-8. Laboratory means for CBNA in Hemp Oil 1 and Hemp Oil 2 (sample/sample

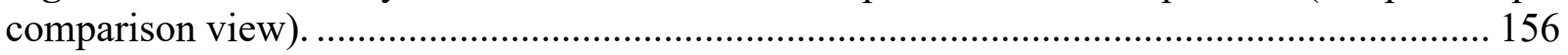

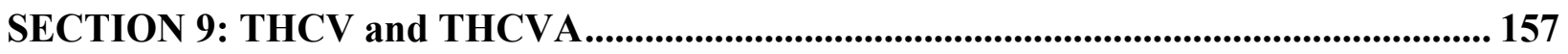

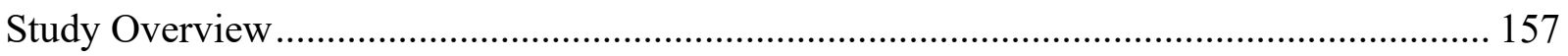

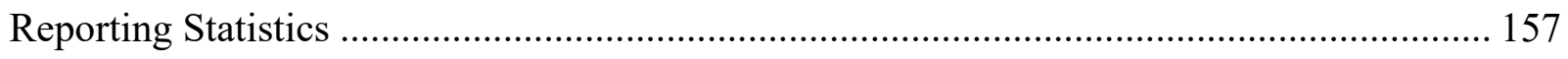

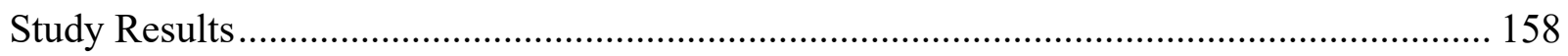

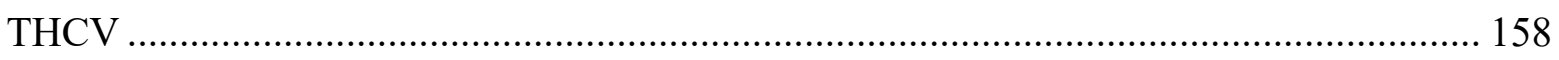

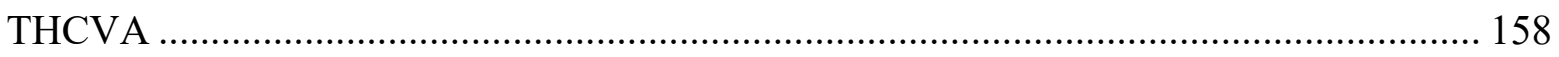

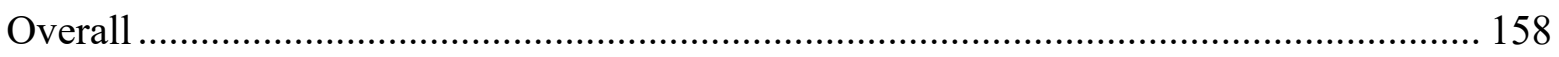

Study Discussion and Technical Recommendations........................................................ 159

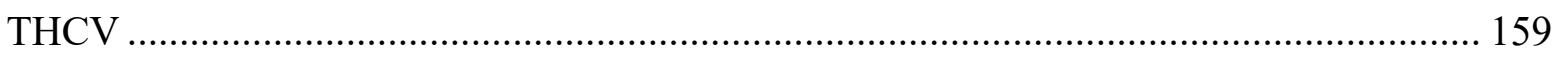

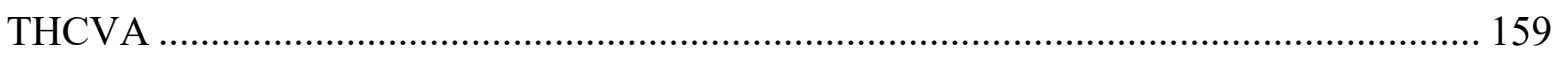

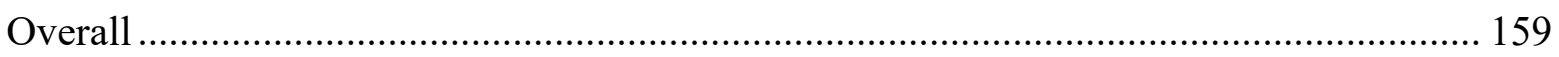

Table 9-1. Individualized data summary table (NIST) for THCV and THCVA in hemp oils. 161

Table 9-2. Data summary table for THCV in hemp oils..................................................... 162 
Figure 9-1. THCV in Hemp Oil 1 (data summary view - analytical method)..................... 164

Figure 9-2. THCV in Hemp Oil 2 (data summary view - analytical method).................... 165

Figure 9-3. THCV in Hemp Oil 2a (data summary view - analytical method)................... 166

Figure 9-4. Laboratory means for THCV in Hemp Oil 1 and Hemp Oil 2 (sample/sample

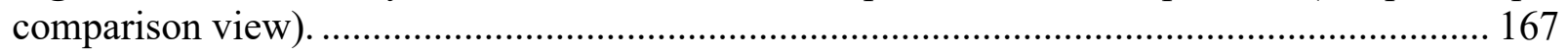

Table 9-3. Data summary table for THCVA in hemp oils. ......................................... 168

Figure 9-5. THCVA in Hemp Oil 1 (data summary view - analytical method).................. 169

Figure 9-6. THCVA in Hemp Oil 2 (data summary view - analytical method).................. 170

Figure 9-7. THCVA in Hemp Oil 2a (data summary view - analytical method)................ 171

Figure 9-8. Laboratory means for THCVA in Hemp Oil 1 and Hemp Oil 2 (sample/sample

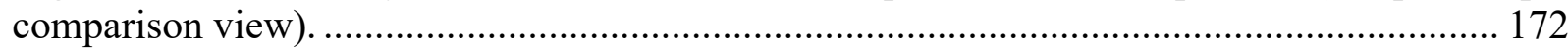




\section{LIST OF ACRONYMS}

\begin{tabular}{|c|c|c|}
\hline & $\mathrm{ACN}$ & Acetonitrile \\
\hline & $\mathrm{CBC}$ & Cannabichromene \\
\hline & $\mathrm{CBCA}$ & Cannabichromenic Acid \\
\hline & CBD & Cannabidiol \\
\hline & CBDA & Cannabidiolic acid \\
\hline & CBDV & Cannabidivarin \\
\hline & CBDVA & Cannabidivarinic Acid \\
\hline & CBG & Cannabigerol \\
\hline & CBGA & Cannabigerolic Acid \\
\hline & CBL & Cannabicyclol \\
\hline & CBLA & Cannabicyclolic Acid \\
\hline & $\mathrm{CBN}$ & Cannabinol \\
\hline & CBNA & Cannabinolic Acid \\
\hline & cGMP & current Good Manufacturing Practice \\
\hline & $\mathrm{CO}_{2}$ & Carbon dioxide \\
\hline P & CRM & Certified Reference Material \\
\hline & GC-FID & Gas Chromatography with Flame Ionization Detection \\
\hline & GC-MS & Gas Chromatography Mass Spectrometry \\
\hline$\exists$ & CannaQAP & Cannabis Quality Assurance Program \\
\hline & DSQAP & Dietary Supplements Laboratory Quality Assurance Program \\
\hline$\dot{5}$ & HAMQAP & Health Assessment Measurements Quality Assurance Program \\
\hline & $\mathrm{H}_{2} \mathrm{O}$ & Water \\
\hline & LC-UV & Liquid Chromatography with UV Absorbance Detection \\
\hline & LC-PDA & Liquid Chromatography with Photodiode Array Detection \\
\hline & LC-MS & Liquid Chromatography with Mass Spectrometry Detection \\
\hline $\bar{b}$ & LC-MS/MS & Liquid Chromatography with Tandem Mass Spectrometry Detection \\
\hline 2 & LOQ & Limit of Quantification \\
\hline$x$ & $\mathrm{MeOH}$ & Methanol \\
\hline$x^{2}$ & NA & Not available \\
\hline & NIST & National Institute of Standards and Technology \\
\hline $\bar{\partial}$ & PA & Phosphoric Acid \\
\hline 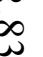 & QAP & Quality Assurance Program \\
\hline & $\mathrm{QL}$ & Quantification Limit \\
\hline & $\mathrm{RM}$ & Reference Material \\
\hline & RSD & Relative Standard Deviation \\
\hline & SD & Standard Deviation \\
\hline & SRM & Standard Reference Material \\
\hline & $\mathrm{THC}$ & Tetrahydrocannabinol \\
\hline & $\Delta^{8}-\mathrm{THC}$ & $\Delta^{8}$-Tetrahydrocannabinol \\
\hline & $\Delta^{9}-\mathrm{THC}$ & $\Delta^{9}$-Tetrahydrocannabinol \\
\hline & THCA & Tetrahydrocannabinolic acid \\
\hline & THCV & Tetrahydrocannabivarin \\
\hline & THCVA & Tetrahydrocannabivarinic Acid \\
\hline
\end{tabular}




\begin{abstract}
NIST launched a CannaQAP in 2020 to improve the comparability of the analytical measurements in forensic and Cannabis (hemp and marijuana) testing laboratories. CannaQAP is an interlaboratory study mechanism that is similar to a proficiency testing scheme; however, the focus is towards education without assigning pass/fail grades to anonymized participants. CannaQAP helps inform NIST about the current measurement capabilities and challenges to assist in the design and characterization of Cannabis RMs. Exercise 1 of CannaQAP focused on the determination of cannabinoids including $\Delta^{9}$-THC, THCA, total THC, CBD, CBDA, total CBD, and up to 13 additional cannabinoids in two hemp oils. This report provides a detailed description of the results of this exercise.
\end{abstract}

\title{
INTRODUCTION
}

CannaQAP offers the opportunity for laboratories to assess their in-house measurements of cannabinoids, other desirable components (e.g., terpenes), and contaminants (e.g., toxic elements, mycotoxins) in samples distributed by NIST. Reports and certificates of participation are provided and may be used as part of their laboratory's validation scheme, demonstrate compliance with cGMPs, and to potentially fulfill proficiency requirements established by related accreditation bodies. In addition, CannaQAP is designed to support the development and dissemination of analytical methods and reference materials. In the future, results from CannaQAP exercises could be used by NIST to identify problematic matrices and analytes for which consensus-based methods of analysis would benefit the stakeholders in numerous Cannabis communities.

NIST has decades of experience in the administration of QAPs, and CannaQAP builds on the approach taken by the former DSQAP and current HAMQAP by emphasizing emerging and challenging measurements in the various Cannabis and Cannabis-derived matrices. QAPs can be viewed as a perpetual interlaboratory study mechanism that is akin to a proficiency testing scheme but without the pass/fail grade with the goal of improving measurement comparability and/or competence for the participant and NIST results. These improvements focus around identifying biases among the different sample extraction, analytical methods and/or calibration approaches. In areas where few standard methods have been recognized, CannaQAP offers a unique tool for assessment of the quality of measurements and provides feedback about performance that can assist participants in improving laboratory operations.

This report summarizes the results from the first exercise of CannaQAP. One hundred sixteen laboratories responded to the call for participants distributed in August 2020. Samples were shipped to participants in October 2020 and results were returned to NIST by November 2020. This report contains the final data and information that was disseminated to the participants in December 2020. The results of the study are summarized below in a series of tables, figures, and text, and reported by section for 17 cannabinoids, total THC, and total CBD. 


\section{OVERVIEW OF DATA TREATMENT AND REPRESENTATION}

Individualized data tables and certificates are provided to the participants that have submitted data, in addition to this report. Examples of the data tables using NIST data are also included in each section of this report. Community tables and figures are provided using randomized laboratory codes, with identities known only to NIST and individual laboratories. The statistical approaches are outlined below for each type of data representation.

\section{$\underline{\text { Statistics }}$}

Data tables and figures throughout this report contain information about the performance of each laboratory relative to that of the other participants in this study and relative to a target around the expected result, if available. All calculations are performed in PROLab Plus (QuoData GmbH, Dresden, Germany). ${ }^{1}$ The consensus means and standard deviations are calculated according to the robust Q/Hampel method outlined in ISO 13528:2015, Annex C. ${ }^{2}$

\section{Individualized Data Table}

The data in this table is individualized to each participating laboratory and is provided to allow participants to directly compare their data to the summary statistics (consensus or community data as well as NIST certified, non-certified, or estimated values, when available). The upper left of the data table includes the randomized laboratory code. Example individualized data tables are included in this report using sample NIST data; participating laboratories received uniquely coded individualized data tables in a separate distribution.

Section 1 of the data table (Your Results) contains the laboratory results as reported, including the mean and standard deviation when multiple values were reported. A blank indicates that NIST does not have data on file for that laboratory for the corresponding analyte or matrix. An empty box for standard deviation indicates that the participant reported a single value or a value below the LOQ and therefore that value was not included in the calculation of the consensus data. ${ }^{2}$ Example individualized data tables are included in this report using NIST data in Section 1 to protect the identity and performance of participants.

Also included in Section 1 are two Z-scores. The first Z-score, $Z^{\prime}$ comm, is calculated with respect to the community consensus value, taking into consideration bias that may result from the uncertainty in the assigned consensus value, using the consensus mean $\left(\mathrm{x}^{*}\right)$, consensus standard deviation $\left(\mathrm{s}^{*}\right)$, and standard deviation for proficiency assessment (SDPA, $\sigma_{P T}^{2}$ ) determined from the $\mathrm{Q} / \mathrm{Hampel}$ estimator:

$$
Z^{\prime}{ }_{\text {comm }}=\frac{x_{i}-x *}{\sqrt{\sigma_{P T^{2}}^{2}+s^{* 2}}}
$$

\footnotetext{
${ }^{1}$ Certain commercial equipment, instruments, or materials are identified in this certificate to adequately specify the experimental procedure. Such identification does not imply recommendation or endorsement by the National Institute of Standards and Technology, nor does it imply that the materials or equipment identified are necessarily the best available for the purpose.

${ }^{2}$ ISO 13528:2015, Statistical methods for use in proficiency testing by interlaboratory comparisons, pp. 53-54.
} 
The second Z-score, $Z_{\text {NIST, }}$ is calculated with respect to the target value (NIST certified, non-certified, or estimated value, when available), using $x_{\mathrm{NIST}}$ and $2 * U_{95}$ (the expanded uncertainty on the certified or reference value, $U_{95}$, or twice the standard deviation of NIST or other measurements):

$$
Z_{\mathrm{NIST}}=\frac{x_{i}-x_{\mathrm{NIST}}}{2 * U_{95}}
$$

or

$$
Z_{\mathrm{NIST}}=\frac{x_{i}-x_{\mathrm{NIST}}}{2 * U_{\mathrm{NIST}}}
$$

The significance of the $Z$-score and $Z^{\prime}$-score is as follows:

- $|Z|<2$ indicates that the laboratory result is considered to be within the community consensus range (for $Z^{\prime}$ comm) or NIST target range (for $Z_{\text {NIST }}$ ).

- $2<|Z|<3$ indicates that the laboratory result is considered to be marginally different from the community consensus value (for $Z^{\prime}$ comm) or NIST target value (for $Z_{\mathrm{NIST}}$ ).

- $|Z|>3$ indicates that the laboratory result is considered to be significantly different from the community consensus value (for $Z^{\prime}$ comm) or NIST target value (for $Z_{\mathrm{NIST}}$ ).

Section 2 of the data table (Community Results) contains the consensus results, including the number of laboratories reporting more than a single quantitative value for each analyte, the mean value determined for each analyte, and a robust estimate of the standard deviation of the reported values. ${ }^{2}$ Consensus means and standard deviations are calculated using the laboratory means; if a laboratory reported a single value, the reported value is not included in determination of the consensus values. ${ }^{3}$ Additional information on calculation of the consensus mean and standard deviation can be found in the previous section.

Section 3 of the data table (Target) contains the target values for each analyte, when available. When possible, the target value is a certified value, a non-certified value, or a value determined at NIST. In this study, target values were determined at NIST through a validated LC-PDA method summarized in the Study Material Preparation and Characterization Section below. The target values for Hemp Oil 1 and Hemp Oil 2 represent the mean of at least three tested samples with triplicate preparations from the sample package. The target values for Hemp Oil 2 represent the mean of at least three tested samples from different sample package. These measurements allowed for the NIST to provide an expanded uncertainty $\left(U_{95}\right)$ to encompass variability due to inhomogeneity within and between packaged units.

\section{Summary Data Table}

This data table includes a summary of all reported data for a particular analyte in a particular study. Participants can compare the raw data for their laboratory to data reported by the other participating laboratories and to the consensus data. A blank indicates that the laboratory signed up and received samples for that analyte and matrix, but NIST does not have data on file for that laboratory. Data highlighted in red have been flagged as a data entry of zero or results that include text (e.g., "<LOQ" or "present"). Data highlighted in blue have been identified as outside the consensus tolerance limits and would be estimated to yield $\mid Z^{\prime}$ comm $\mid>2$. 


\section{Figures}

\section{Data Summary View (Method Comparison Data Summary View)}

In this view, individual laboratory data (circles) are plotted with the individual laboratory standard deviation (rectangle). Laboratories reporting values below the LOQ are shown in this view as downward triangles beginning at the LOQ, reported as QL on the figures. Laboratories reporting values as "below LOQ" can still be successful in the study if the target value is also below the laboratory LOQ. The blue solid line represents the consensus mean, and the green shaded area represents the $95 \%$ confidence interval for the consensus mean, based on the standard error of the consensus mean. The uncertainty in the consensus mean is calculated using the equation below, based on the repeatability standard deviation $\left(s_{r}\right)$, the reproducibility standard deviation $\left(s_{R}\right)$, the number of participants reporting data, and the average number of replicates reported by each participant. The uncertainty about the consensus mean is independent of the range of tolerance. Where appropriate, two consensus means may be calculated for the same sample if bimodality is identified in the data. In this case, two consensus means and ranges will be displayed in the data summary view.

$$
u_{\text {mean }}=\sqrt{\frac{s_{R}^{2}-s_{r}^{2}}{n_{\text {particpants }}}+\frac{s_{R}^{2}}{n_{\text {participants }} \times n_{\text {Average Number of Replicates per Participant }}}}
$$

The red shaded region represents the target zone for "acceptable" performance, which encompasses the NIST target value bounded by twice its uncertainty ( $U_{95}$ or $U_{\text {NIST }}$ ). The solid red lines represent the range of tolerance (values that result in an acceptable $Z^{\prime}$ score, $\left|Z^{\prime}\right| \leq 2$ ). If the lower limit is below zero, the lower limit has been set to zero. In this view, the relative locations of individual laboratory data and consensus zones with respect to the target zone can be compared easily. In most cases, the target zone and the consensus zone overlap, which is the expected result. Major program goals include both reducing the size of the consensus zone and centering the consensus zone about the target value. Analysis of an appropriate reference material as part of a quality control scheme can help to identify sources of bias for laboratories reporting results that are significantly different from the target zone. In the case in which a method comparison is relevant, different colored data points may be used to identify laboratories that used a specific approach to sample preparation, analysis, or quantitation.

\section{Sample/Sample Comparison View}

In this view, the individual laboratory results for one sample (e.g., NIST SRM with a certified, NIST RM with non-certified, or NIST-determined value; a less challenging matrix) are compared to the results for another sample (e.g., NIST RM with a more challenging matrix; a commercial sample). The solid red box represents the target zone for the first sample (x-axis) and the second sample (y-axis), if available. The dotted blue box represents the consensus zone for the first sample (x-axis) and the second sample (y-axis). The axes of this graph are centered about the consensus mean values for each sample or control, to a limit of twice the range of tolerance (values that result in an acceptable $Z^{\prime}$ score, $\left|Z^{\prime}\right| \leq 2$ ). Depending on the variability in the data, the axes may be scaled proportionally to better display the individual data points for each laboratory. In some cases, when the consensus and target ranges have limited overlap, the solid red box may only appear partially on the graph. If the variability in the data is high (greater than $100 \%$ RSD), the dotted blue box may also only appear partially on the graph. These views emphasize trends in the data 
that may indicate potential calibration issues or method biases. One program goal is to identify such calibration or method biases and assist participants in improving analytical measurement capabilities. In some cases, when two equally challenging materials are provided, the same view (sample/sample comparison) can be helpful in identifying commonalities or differences in the analysis of the two materials. 


\section{SECTION 1: STUDY MATERIAL PREPARATION AND CHARACTERIZATION}

NIST Method for Study Material Characterization

\section{Analytical Method}

All study materials were characterized at NIST using an LC-PDA (Cannabis Analyzer, Shimadzu Scientific Instruments, Columbia, MD, USA) equipped with a binary pump, degasser, autosampler, column compartment, and a photodiode array detector, controlled using commercial Lab Solutions software (Shimadzu Scientific Instruments). Cannabinoid separations were carried out on a NexLeaf CBX for Potency C18 column (Shimadzu Scientific Instruments) with $15.0 \mathrm{~cm}$ length, $4.6 \mathrm{~mm}$ inner diameter, and $2.7 \mu \mathrm{m}$ average particle diameter, protected by installation of a NexLeaf CBX guard column (Shimadzu Scientific Instruments). Premixed mobile phase solvents $\left[\mathrm{H}_{2} \mathrm{O}\right.$ and $\mathrm{ACN}$ containing $0.085 \% \mathrm{PA}$ (volume ratios)] were obtained from Shimadzu Scientific Instruments. The separation and detection conditions are summarized below.

$\underline{\text { Parameters }}$

Injection Volume

Column Temperature

Flow rate

Mobile Phase Program

$$
\begin{gathered}
\frac{\text { Settings }}{5 \mu \mathrm{L}} \\
40^{\circ} \mathrm{C} \\
1.6 \mathrm{~mL} / \mathrm{min}
\end{gathered}
$$

\begin{tabular}{c} 
Time $(\min )$ \\
\hline 0.0 \\
3.0 \\
7.0 \\
7.1 \\
8.0 \\
8.1 \\
10.0
\end{tabular}

Absorbance Wavelength Range

$0.085 \%$ PA in $\mathrm{H}_{2} \mathrm{O}$
30
30
15
5
5
30
30

$\frac{0.085 \% \mathrm{PA} \text { in } \mathrm{ACN}}{70}$

70

85

95

95

70

70

\section{Calibration}

A CRM solution of 11 cannabinoids in ACN was obtained from Shimadzu Scientific Instruments. The mass concentration of each cannabinoid in the solution was $250 \mathrm{mg} / \mathrm{L}$. Three independent working calibration solutions were gravimetrically prepared to have final mass concentrations of $2.5 \mathrm{mg} / \mathrm{L}, 10 \mathrm{mg} / \mathrm{L}$, and $25 \mathrm{mg} / \mathrm{L}$ of each cannabinoid. The working solutions were analyzed by the LC-PDA method summarized above using a common wavelength of $220 \mathrm{~nm}$. Peak areas were plotted for each compound with its corresponding mass concentration to construct an external calibration curve. Triplicate injection of the calibration standards demonstrated adequate reproducibility of the chromatographic method with RSDs below $3 \%$ for all cannabinoids.

\section{Hemp Oil 1}

\section{Study Material Preparation}

Hemp Oil 1 was prepared by CV Sciences (San Diego, CA, USA) for use in CannaQAP through a $\mathrm{CO}_{2}$ extraction from certified food-fiber hemp seed with decarboxylation to convert CBDA to $\mathrm{CBD}$. The material was packaged into $10 \mathrm{~mL}$ amber vials and stored under controlled refrigeration $\left(\approx 4{ }^{\circ} \mathrm{C}\right)$ until shipment to NIST. Samples of the material were sent to Alkemist Labs (Garden Grove, CA, USA) for cannabinoid testing prior to shipment to NIST. Upon arrival at NIST, 
materials were stored under controlled refrigeration $\left(\approx 4{ }^{\circ} \mathrm{C}\right)$ until shipment to participating laboratories.

\section{Study Material Characterization}

Samples were prepared following the approach of Vaclavik et al., ${ }^{3}$ modified to use $\mathrm{MeOH}$ instead of ethanol. Three $0.5 \mathrm{~g}$ samples of three individual Hemp Oil 1 sample bottles $(\mathrm{N}=9)$ were accurately weighed into $50 \mathrm{~mL}$ centrifuge tubes and diluted with $\approx 20.0 \mathrm{~g}$ of $\mathrm{MeOH}$. Samples were vortexed for $10 \mathrm{~s}$ to ensure initial mixing and shaken for $15 \mathrm{~min}$ using a large benchtop capacity mixer from Glas-Col (Terre Haute, IN, USA). Small quantities of Hemp Oil 1 samples were further diluted with $\mathrm{MeOH}$ resulting in a 10-fold and 100-fold dilutions. The original and diluted samples were filtered using a $0.45 \mu \mathrm{m}$ PFTE syringe filter into autosampler vials for analysis by LC-PDA.

The LC-PDA chromatograms at $220 \mathrm{~nm}$ for samples of Hemp Oil 1 with no additional dilution, 10-fold dilution, and 100-fold dilution are shown in Figure 1-1. CBDV, CBG, CBD, CBN, $\Delta^{9}$-THC, and CBC were tentatively identified in Hemp Oil 1 based on matching retention times to reference standards. The presence of CBDV, CBG, CBD, CBN, $\Delta^{9}-\mathrm{THC}$, and CBC was confirmed by comparison of the absorbance spectra collected at the maximum of the chromatographic peaks in Hemp Oil 1 to those collected from reference standards (Figure 1-2). With the exception of $\mathrm{CBC}$, the absorbance spectra correlated extremely well between the samples and the standards. The absorbance spectrum of $\mathrm{CBC}$ in the calibrant is more distinct than the other five cannabinoids, with clear spectral features at $193 \mathrm{~nm}, 230 \mathrm{~nm}$, and $284 \mathrm{~nm}$. The absorbance spectrum of the chromatographic peak at 7.1 min contains features at similar absorbance wavelengths, but with drastically different peak heights indicating the coelution of interfering species in the chromatographic peak.

To investigate the potential interference in the CBC chromatographic peak for Hemp Oil 1, the extracted chromatogram at $220 \mathrm{~nm}$ was enlarged in Figure 1-3A. Absorbance spectra collected at $7.059 \mathrm{~min}, 7.113 \mathrm{~min}$, and $7.200 \mathrm{~min}$ are compared to the reference standard in Figure 1-3B, Figure 1-3C, and Figure 1-3D, respectively. The absorbance spectrum obtained from Hemp Oil 1 at $7.059 \mathrm{~min}$ is virtually identical to $\mathrm{CBC}$, with some variability in signal intensity. The absorbance spectrum at the chromatographic peak maximum in Hemp Oil 1 (7.113 min) has some representation of $\mathrm{CBC}$ with a 5-fold higher signal intensity but also includes a more intense spectral peak at $195 \mathrm{~nm}$. The absorbance spectrum at $7.200 \mathrm{~min}$ from the Hemp Oil 1 sample shows contribution from the $195 \mathrm{~nm}$ peak only. Similar observations were noted for Hemp Oil 2 and 2a samples (data not shown). These results clearly indicate that $\mathrm{CBC}$ is present in the three samples, but that CBC cannot be quantitatively measured by LC-PDA using the $220 \mathrm{~nm}$ wavelength. Impact of the wavelength selected for quantitation of $\mathrm{CBC}$ is demonstrated in Figure $\mathbf{1 - 4}$, and as a result, $230 \mathrm{~nm}$ was used as the detection wavelength for CBC for the three hemp oil samples.

A summary of the determined mass fraction (\%) values for CBDV, CBG, CBD, CBN, $\Delta^{9}-\mathrm{THC}$, and CBC in Hemp Oil 1 are summarized below. The good precision of the LC-PDA measurements with RSDs at or below $\approx 6 \%$ between bottles indicating that Hemp Oil 1 samples were sufficiently

\footnotetext{
${ }^{3}$ L Vaclavik, F Benes, M Fenclova, J Hricko, A Krmela, V Svobodova, J Hajslova, K Mastovska. J AOAC Int 102(6):
} 1822-1833 (2019) https://doi.org/10.1093/jaoac/102.6.1822. 
homogeneous for use as study samples in CannaQAP Exercise 1. These values will be used as target values to estimate accuracy of participant results ( $\left.Z_{\mathrm{NIST}}\right)$.

\begin{tabular}{c} 
Cannabinoids \\
\hline CBDV \\
CBG \\
CBD \\
CBN \\
$\Delta^{9}-$ THC \\
CBC
\end{tabular}

Mean $\pm \mathrm{SD}$
$0.0391 \pm 0.0024$
$0.0636 \pm 0.0025$
$4.310 \pm 0.058$
$0.01582 \pm 0.00048$
$0.1315 \pm 0.0026$
$0.1975 \pm 0.0023$

\begin{tabular}{c} 
RSD (\%) \\
\hline 6.17 \\
3.91 \\
1.34 \\
3.04 \\
1.98 \\
1.30
\end{tabular}

\section{Participant Instructions}

Participants were provided with one bottle containing approximately $5 \mathrm{~mL}$ of hemp oil and were asked to store the sample under controlled refrigeration $\left(\approx 4^{\circ} \mathrm{C}\right)$. Before use, participants were instructed to allow the contents of the bottle to equilibrate at room temperature for $24 \mathrm{~h}$ before mixing thoroughly. A sample size of $0.5 \mathrm{~g}$ was recommend based on homogeneity measurements at NIST to help minimize variability caused by sampling in the end results. Participants were asked to prepare three samples and report three mass fraction (\%) values from the single bottle provided on an as-received basis.

\section{Hemp Oil 2}

Study Material Preparation

Hemp Oil 2 was prepared by CV Sciences (San Diego, CA, USA) for use in CannaQAP by distillation of Hemp Oil 1 and dilution with additional hemp oil fractions to reduce the mass fraction of total THC to below $0.3 \%$. The material was packaged into $10 \mathrm{~mL}$ amber vials and stored under controlled refrigeration $\left(\approx 4^{\circ} \mathrm{C}\right)$ until shipment to NIST. Samples of the material were sent to Alkemist Labs (Garden Grove, CA, USA) for cannabinoid testing prior to shipment to NIST. Upon arrival at NIST, materials were stored under controlled refrigeration $\left(\approx 4{ }^{\circ} \mathrm{C}\right)$ until shipment to participating laboratories.

\section{Study Material Characterization}

Samples were prepared following the approach of Vaclavik et al., ${ }^{3}$ modified to use $\mathrm{MeOH}$ instead of ethanol. Three $0.5 \mathrm{~g}$ samples of three individual Hemp Oil 2 sample bottles $(\mathrm{N}=9)$ were accurately weighed into $50 \mathrm{~mL}$ centrifuge tubes and diluted with $\approx 20.0 \mathrm{~g}$ of $\mathrm{MeOH}$. Samples were vortexed for $10 \mathrm{~s}$ to ensure initial mixing and shaken for $15 \mathrm{~min}$ using a large benchtop capacity mixer from Glas-Col (Terre Haute, IN, USA). Small quantities of Hemp Oil 2 samples were further diluted with $\mathrm{MeOH}$ resulting in a 10-fold and 100-fold dilutions. The original and diluted samples were filtered using a $0.45 \mu \mathrm{m}$ PFTE syringe filter into autosampler vials for analysis by LC-PDA.

The LC-PDA chromatograms at $220 \mathrm{~nm}$ for samples of Hemp Oil 2 with no additional dilution, 10-fold dilution, and 100-fold dilution are shown in Figure 1-5. CBDV, CBG, CBD, CBN, $\Delta^{9}$-THC, and $\mathrm{CBC}$ were identified and determined in Hemp Oil 2 using similar procedures as described for Hemp Oil 1.

A summary of the determined mass fraction (\%) values for CBDV, CBG, CBD, CBN, $\Delta^{9}-\mathrm{THC}$, and $\mathrm{CBC}$ in Hemp Oil 2 are summarized below. The good precision of the LC-PDA measurements 
with RSDs at or below $5 \%$ between bottles indicated that Hemp Oil 2 samples were sufficiently homogeneous for use as study samples in CannaQAP Exercise 1. These values will be used as target values to estimate accuracy of participant results ( $\left.Z_{\mathrm{NIST}}\right)$.

\begin{tabular}{c} 
Cannabinoids \\
\hline CBDV \\
CBG \\
CBD \\
CBN \\
$\Delta^{9}-\mathrm{THC}$ \\
CBC
\end{tabular}

$$
\begin{gathered}
\text { Mean } \pm \text { SD } \\
0.1422 \pm 0.0038 \\
0.0879 \pm 0.0041 \\
9.21 \pm 0.27 \\
0.0240 \pm 0.0011 \\
0.1604 \pm 0.0046 \\
0.4122 \pm 0.0056
\end{gathered}
$$

\begin{tabular}{c} 
RSD (\%) \\
\hline 2.64 \\
4.71 \\
2.90 \\
4.78 \\
2.84 \\
1.37
\end{tabular}

\section{Participant Instructions}

Participants were provided with one bottle containing approximately $5 \mathrm{~mL}$ of hemp oil and were asked to store the sample under controlled refrigeration $\left(\approx 4^{\circ} \mathrm{C}\right)$. Before use, participants should mix the sample thoroughly after allowing the contents of the bottle to equilibrate at room temperature for $3 \mathrm{~h}$, which was shorter than Hemp Oil 1 because the sample was a finished product sample matrix. A sample size of $0.5 \mathrm{~g}$ was recommend based on homogeneity measurements at NIST to help minimize variability caused by sampling in the end results. Participants were asked to prepare three samples and report three mass fraction (\%) values from the single bottle provided on an as-received basis.

\section{Hemp Oil 2a}

\section{Study Material Preparation}

Hemp Oil 2a was prepared through the dilution of Hemp Oil $2(1.60508 \mathrm{~g})$ with methanol $(62.41220 \mathrm{~g})$ and ethanol $(10.49705 \mathrm{~g})$ with shaking for $30 \mathrm{~min}$ using a large benchtop capacity mixer from Glas-Col (Terre Haute, IN, USA) and filtration to remove any undissolved precipitates. The material was packaged into $1.8 \mathrm{~mL}$ amber autosampler vials and stored at $\approx 4{ }^{\circ} \mathrm{C}$ until shipment to participating laboratories.

\section{Study Material Characterization}

Small quantities of three Hemp Oil 2a samples were further diluted with $\mathrm{MeOH}$ resulting in a 10fold and 100-fold dilutions. The original and diluted samples were filtered using a $0.45 \mu \mathrm{m}$ PFTE syringe filter into autosampler vials for analysis by LC-PDA.

The LC-PDA chromatograms at $220 \mathrm{~nm}$ for samples of Hemp Oil 2a with no additional dilution, 10-fold dilution, and 100-fold dilution are shown in Figure 1-6. CBDV, CBG, CBD, CBN, $\Delta^{9}$-THC, and CBC were identified and determined in Hemp Oil 2a using similar procedures as described for Hemp Oil 1 and Hemp Oil 2.

A summary of the determined mass fraction (\%) values for CBDV, CBG, CBD, CBN, $\Delta^{9}-\mathrm{THC}$, and $\mathrm{CBC}$ in Hemp Oil $2 \mathrm{a}$ are summarized below. The good precision of the LC-PDA measurements with RSDs at or below $\approx 6.5 \%$ between bottles indicated that Hemp Oil 2a samples were sufficiently homogeneous for use as study samples in CannaQAP Exercise 1. These values will be used as target values to estimate accuracy of participant results ( $\left.Z_{\mathrm{NIST}}\right)$. 


\begin{tabular}{c} 
Cannabinoids \\
\hline $\mathrm{CBDV}$ \\
$\mathrm{CBG}$ \\
$\mathrm{CBD}$ \\
$\mathrm{CBN}$ \\
$\Delta^{9}-\mathrm{THC}$ \\
$\mathrm{CBC}$
\end{tabular}

$$
\begin{gathered}
\underline{\text { Mean } \pm \mathrm{SD}} \\
0.1273 \pm 0.0041 \\
0.0904 \pm 0.0022 \\
9.457 \pm 0.038 \\
0.01725 \pm 0.00077 \\
0.1543 \pm 0.0060
\end{gathered}
$$

$\underline{\mathrm{RSD}(\%)}$

3.22

2.45

0.40

4.44

3.90

6.34

$\frac{N}{6}$
3
3
3
6
3

\section{Participant Instructions}

Participants were provided with three sample vials, each containing approximately $1 \mathrm{~mL}$ of hemp oil. Participants were asked to store the samples under controlled refrigeration $\left(\approx 4{ }^{\circ} \mathrm{C}\right)$. Before use, participants were instructed to allow the contents of the bottle to equilibrate at room temperature for $3 \mathrm{~h}$ before mixing thoroughly and removing a sample size appropriate to their method of analysis. Participants were asked to prepare one sample and report one mass fraction (\%) value from each vial provided on an as-received basis and adjusting for the dilution information provided. 


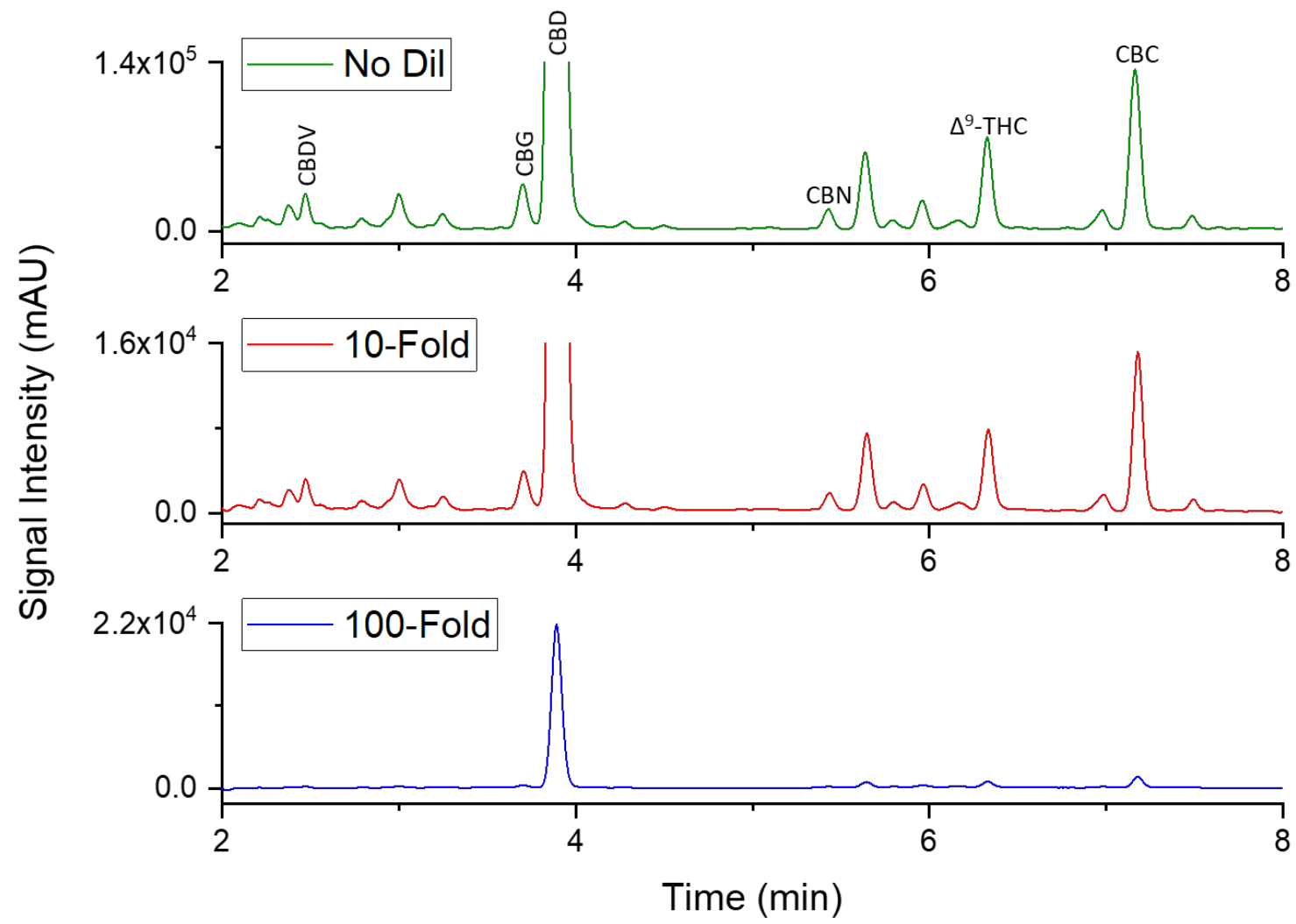

Figure 1-1. Extracted wavelength chromatogram for Hemp Oil 1 at $220 \mathrm{~nm}$. 

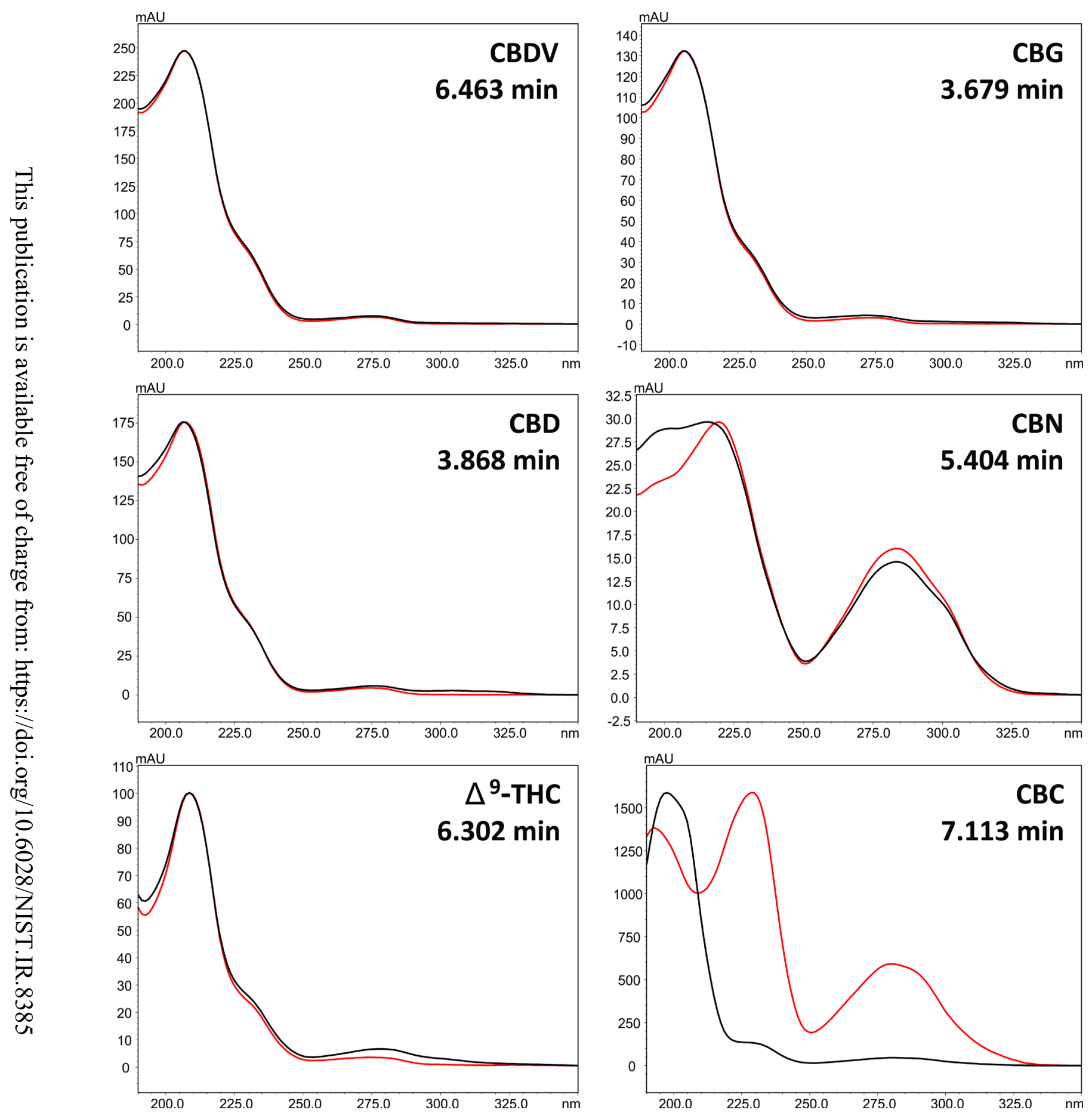

Figure 1-2. UV absorbance spectra of the six suspected cannabinoid peaks in Hemp Oil 2 (black) and calibration standard (red). 

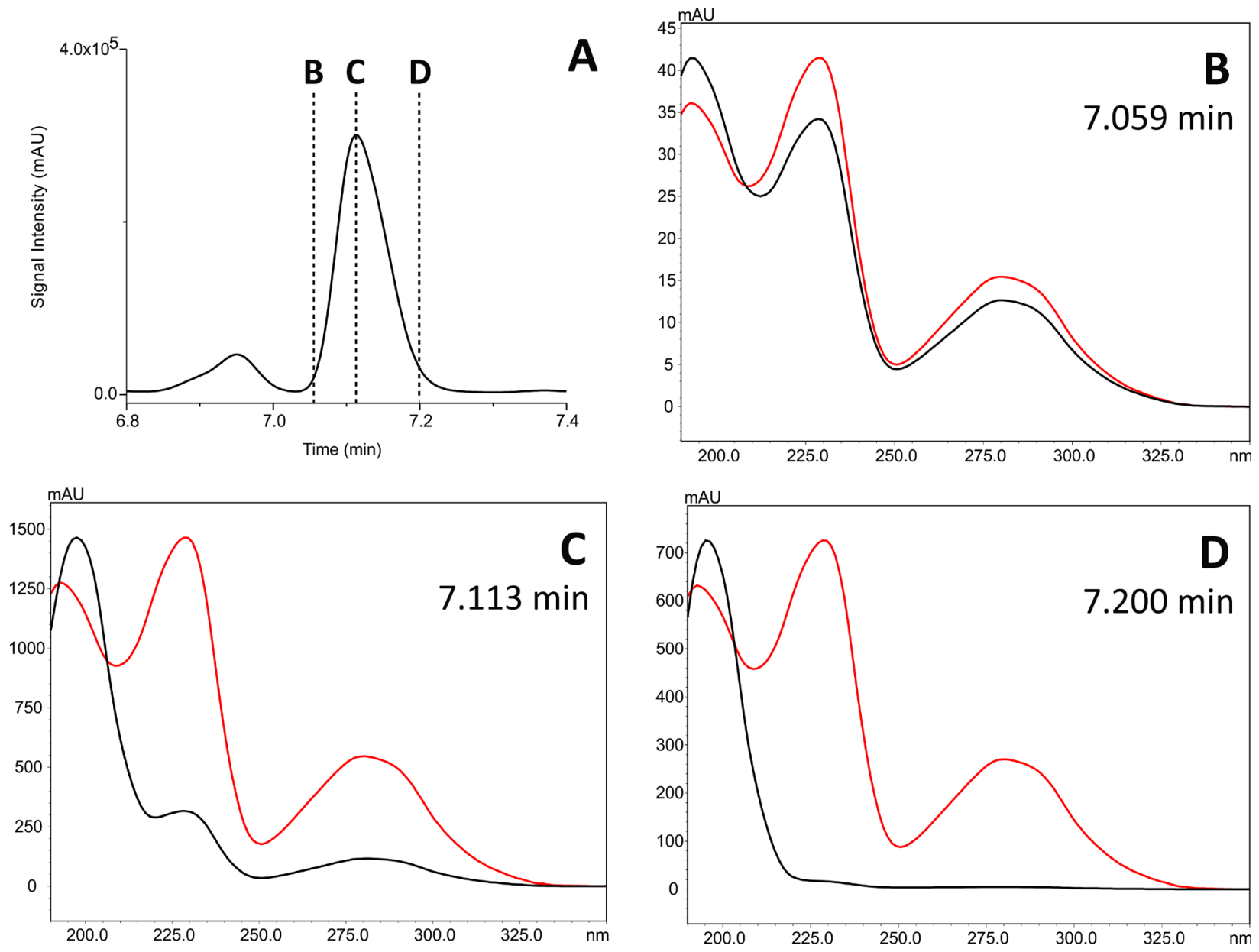

Figure 1-3. (A) Selection of the extracted wavelength chromatogram at $220 \mathrm{~nm}$ for Hemp Oil 2. (B, C, D) UV absorbance spectra of suspected CBC peak in Hemp Oil 2 (black) at different chromatographic retention times compared to a calibration standard (red). 


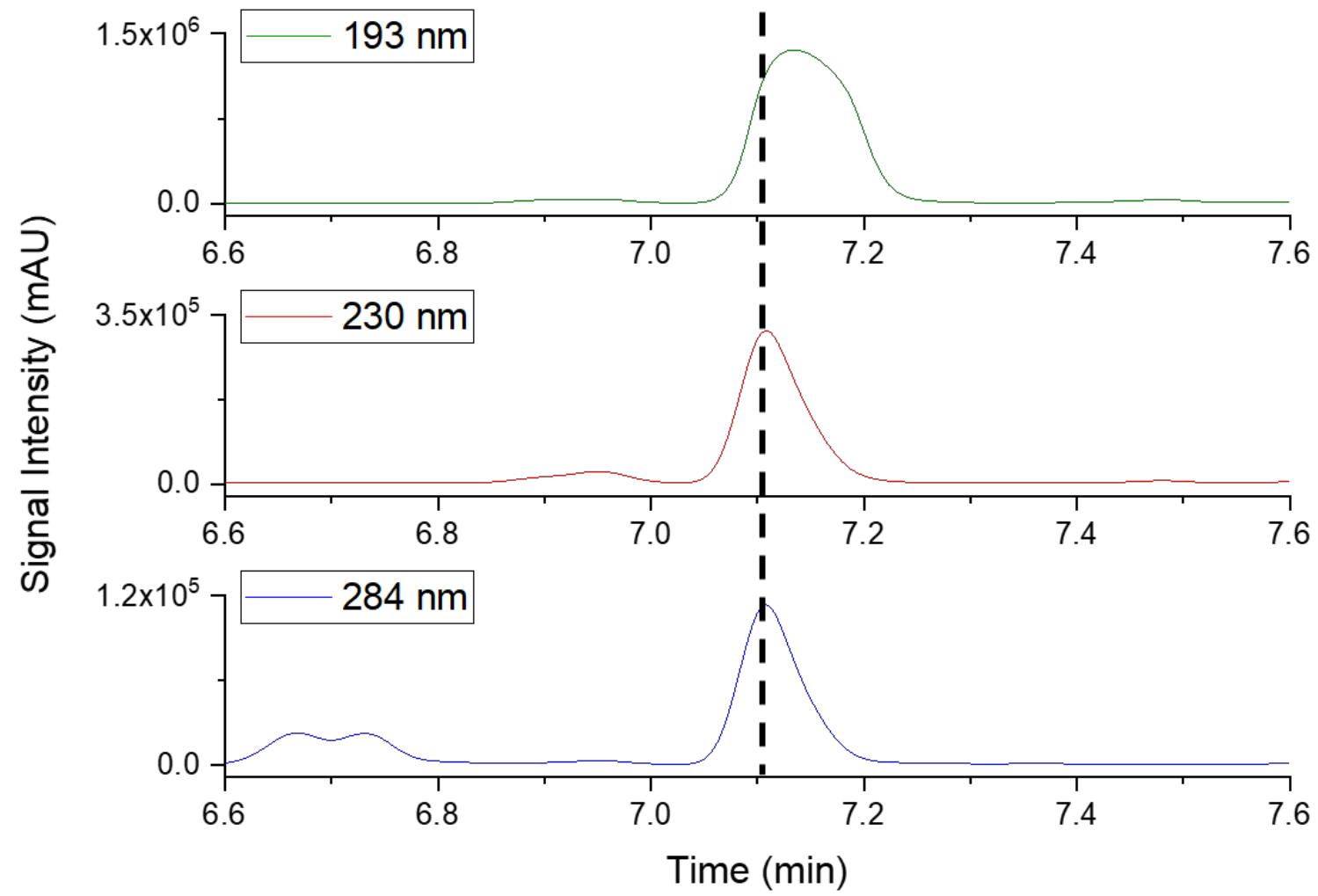

Figure 1-4. Extracted wavelength chromatogram at different wavelengths for the CBC chromatographic peak in Hemp Oil 2. 


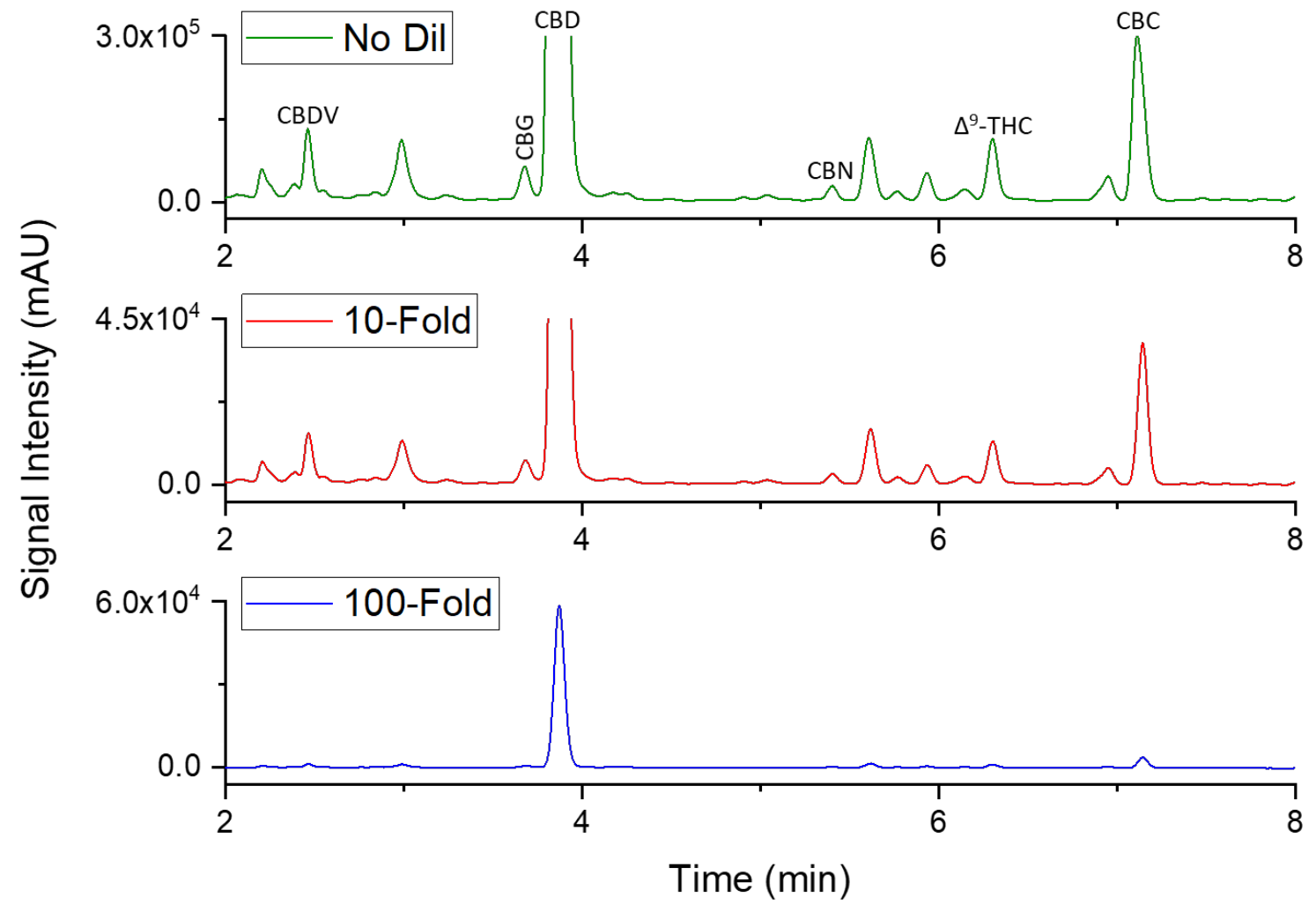

Figure 1-5. Extracted wavelength chromatogram for Hemp Oil 2 at $220 \mathrm{~nm}$. 


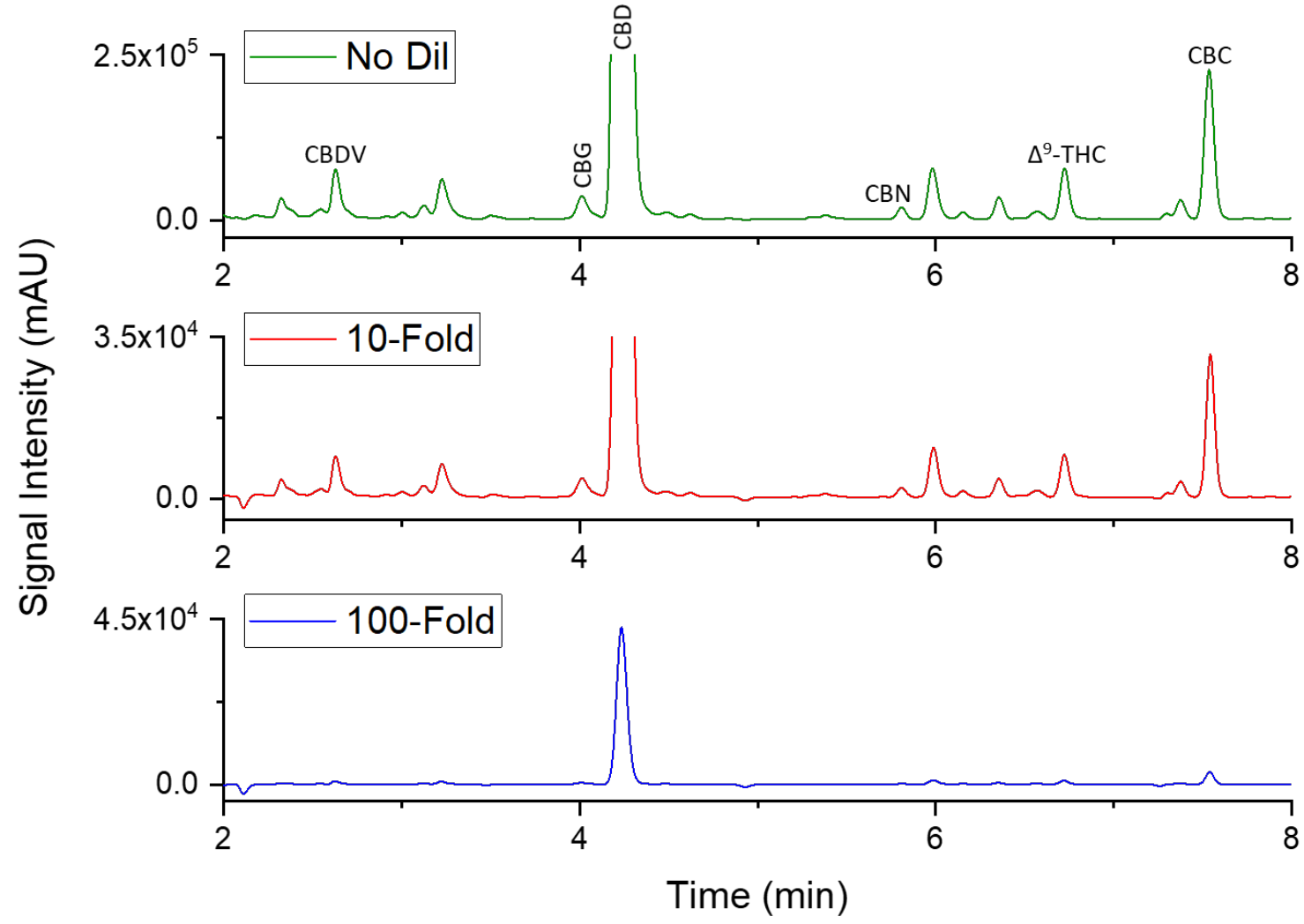

Figure 1-6. Extracted wavelength chromatogram for Hemp Oil 2a at $220 \mathrm{~nm}$. 


\section{SECTION 2: $\Delta^{9}$-THC, THCA, $\Delta^{8}$-THC, AND TOTAL THC}

Study Overview

The medicinal and recreational use of Cannabis (hemp and marijuana) and Cannabis-derived products continues to increase across the United States. As the industry grows, so does the need for reliable differentiation between legal and illegal Cannabis-derived products, which is highly variable depending on local regulations. This need for distinction has motivated a new interest in the analysis of $\Delta^{9}$-THC, THCA, and total THC mass fractions (\%). THCA, the acidic precursor of $\Delta^{9}$-THC, is synthesized in the glandular trichomes of the Cannabis plant and forms $\Delta^{9}$-THC after the parent compound is decarboxylated by UV exposure, prolonged storage, or heat. ${ }^{4}$ Additionally, $\Delta^{8}$-THC, a non-psychoactive stereoisomer of $\Delta^{9}$-THC that may be present in Cannabis and derived products, has similar chromatographic behavior and mass spectral fingerprint to $\Delta^{9}$-THC. Many laboratories are interested in quantitation of $\Delta^{8}-\mathrm{THC}$ to ensure that their analytical methods can appropriately distinguish between the two isomers. Participants in this study were asked to use in-house analytical methods to determine the mass fraction (\%) of $\Delta^{9}-\mathrm{THC}$, THCA, $\Delta^{8}-\mathrm{THC}$, and total THC in three hemp oils. The preparation of these hemp oils included a decarboxylation step resulting in extremely low levels of THCA and levels of $\Delta^{9}$-THC in normal commercial hemp products.

\section{Reporting Statistics}

- The enrollment and reporting statistics for $\Delta^{9}$-THC, THCA, $\Delta^{8}-\mathrm{THC}$, and total THC are described in the table below for each analyte. Reported values may include non-quantitative results (zero or below LOQ).

\begin{tabular}{ccccccc} 
& \multicolumn{2}{c}{ Hemp Oil 1 } & \multicolumn{2}{c}{ Hemp Oil 2 } & & \multicolumn{2}{c}{ Hemp Oil 2a } \\
& $\underline{\text { Number of }}$ & $\underline{\underline{\text { Reporting }}}$ & $\underline{\underline{\text { Number of }}}$ & $\underline{\underline{\text { Reporting }}}$ & $\underline{\underline{\text { Number of }}}$ & $\underline{\underline{\text { Reporting }}}$ \\
$\underline{\text { Analyte }}$ & $\underline{\text { Participants }}$ & $\underline{\text { Results }}$ & $\frac{\text { Participants }}{59}$ & $\underline{\text { Results }}$ & $\underline{\underline{\text { Participants }}}$ & $\underline{\underline{\text { Results }}}$ \\
$\Delta^{9}$-THC & 79 & $86 \%$ & 92 & $85 \%$ & 19 & $63 \%$ \\
THCA & 78 & $69 \%$ & 90 & $72 \%$ & 19 & $47 \%$ \\
$\Delta^{8}$-THC & 56 & $66 \%$ & 66 & $64 \%$ & 19 & $42 \%$ \\
Total THC & 74 & $72 \%$ & 81 & $74 \%$ & 19 & $63 \%$
\end{tabular}

- Most laboratories reported using solvent extraction or sample dilution for determination of $\Delta^{9}$-THC, THCA, $\Delta^{8}$-THC, and total THC in the three hemp oil samples (see table below). Additional sample preparation details are summarized at the end of the report in Appendix I.

\footnotetext{
${ }^{4}$ G Moreno-Sanz. Cannabis and Cannabinoid Research 1(1): 124-130 (2016) http://doi.org/10.1089/can.2016.0008.
} 
Reported

Preparation Method

Solvent Extraction

Dilution

Other

None

No Response

\section{$\underline{\Delta^{9}-\mathrm{THC}}$ \\ 67.3}

24.7

1.9

1.2

4.9
Percent Reporting

\begin{tabular}{|c|c|c|}
\hline$\underline{\mathrm{THCA}}$ & $\Delta^{8}-\mathrm{THC}$ & $\underline{\text { Total THC }}$ \\
\hline 69.1 & 70.4 & 68.6 \\
\hline 22.4 & 22.2 & 21.4 \\
\hline 1.3 & 1.9 & 2.9 \\
\hline 1.3 & 1.9 & 1.4 \\
\hline 5.1 & 3.7 & 5.7 \\
\hline
\end{tabular}

- Most laboratories reported using LC-PDA or LC-UV for the determination of $\Delta^{9}$-THC, THCA, $\Delta^{8}$-THC, and total THC (see table below). Additional method details are summarized at the end of the report in Appendix I.

$\underline{\text { Reported }}$

Analytical Method

LC-PDA

LC-UV

LC-MS

LC-MS/MS

GC-FID

GC-MS

Other

$\frac{\Delta^{9}-\mathrm{THC}}{63.6}$

25.3

1.9

3.7

1.2

3.1

0.0
Percent Reporting

\begin{tabular}{|c|c|c|}
\hline THCA & $\Delta^{8}-\mathrm{THC}$ & Total THC \\
\hline 65.8 & 66.7 & 59.3 \\
\hline 27.0 & 24.1 & 23.6 \\
\hline 2.0 & 1.9 & 1.4 \\
\hline 3.3 & 3.7 & 4.3 \\
\hline 0.0 & 0.0 & 5.7 \\
\hline 2.0 & 1.9 & 5.0 \\
\hline 0.0 & 1.9 & 0.7 \\
\hline
\end{tabular}

Study Results

$\Delta^{9}-T H C$

- The mass fractions (\%) for $\Delta^{9}-$ THC in the hemp oil samples were determined by NIST using LC-PDA as described in Section 1 and are summarized in Table 2-1. These NIST values are used as the target means and ranges summarized in Table 2-2 for comparison to the participant results.

- The target and consensus means and ranges are summarized for $\Delta^{9}$-THC via different analytical methods in Figure 2-1, Figure 2-2, and Figure 2-3, which include data from laboratories submitting two or three results for $\Delta^{9}$-THC. Data from participants submitting only one measurement were included in Table 2-2 but were not included in the calculation of consensus statistics. $^{2}$

- For $\Delta^{9}$-THC in Hemp Oil 1, the consensus range was based on quantitative results from 65 laboratories and completely overlaps the target range (Figure 2-1).

- The individual laboratory means from 37 laboratories (57\% of those reporting results) were outside the NIST range of tolerance for $\Delta^{9}$-THC in Hemp Oil 1.

- The individual laboratory means from 10 laboratories (15\% of those reporting results) were outside the acceptable $Z_{\text {comm }}^{\prime}$ score for $\Delta^{9}$-THC in Hemp Oil 1.

- The threshold or LOQ for 1 of 1 laboratory reporting a qualitative result was below the target mean for $\Delta^{9}$-THC in Hemp Oil 1. 
- For $\Delta^{9}$-THC in Hemp Oil 2, the consensus range was based on quantitative results from 72 laboratories and completely overlaps the target range (Figure 2-2).

- The individual laboratory means from 39 laboratories (54\% of those reporting results) were outside the NIST range of tolerance for $\Delta^{9}$-THC in Hemp Oil 2.

- The individual laboratory means from 8 laboratories (11\% of those reporting results) were outside the acceptable $Z_{\text {comm }}^{\prime}$ score for $\Delta^{9}$-THC in Hemp Oil 2.

- The threshold or LOQ for 1 of 3 laboratories reporting a qualitative result were below the target mean for $\Delta^{9}$-THC in Hemp Oil 2.

- For $\Delta^{9}$-THC in Hemp Oil $2 \mathrm{a}$, the consensus range was based on quantitative results from 11 laboratories and overlaps approximately $85 \%$ of the target range (Figure 2-3).

- The individual laboratory means or thresholds from 6 laboratories $(55 \%$ of those reporting results) were outside the NIST range of tolerance for $\Delta^{9}$-THC in Hemp Oil 2a.

- The individual laboratory mean from 1 laboratory ( $9 \%$ of those reporting results) was outside the acceptable $Z_{\text {comm }}^{\prime}$ score for $\Delta^{9}$-THC in Hemp Oil 2 a.

- No results were reported using thresholds or LOQs for $\Delta^{9}$-THC in Hemp Oil $2 \mathrm{a}$.

- A comparison of individual laboratory means for $\Delta^{9}$-THC in Hemp Oil 1 and Hemp Oil 2 is summarized in Figure 2-4 for laboratories who reported results for both samples.

THCA

- No target means or ranges were provided for THCA in the three hemp oils (Table 2-1).

- The consensus means and ranges for THCA are based on quantitative data from 27 laboratories (Figure 2-5), 34 laboratories (Figure 2-6), and 3 laboratories (Figure 2-7) for Hemp Oil 1, Hemp Oil 2, and Hemp Oil 2a, respectively. Data from participants submitting only one measurement were included in Table 2-3 but were not included in the calculation of consensus statistics. $^{2}$

- A comparison of individual laboratory means for THCA in Hemp Oil 1 and Hemp Oil 2 is summarized in Figure 2-8 for laboratories who reported results for both samples.

$\Delta^{8}-T H C$

- No target means or ranges were provided for $\Delta^{8}$-THC in the three hemp oils (Table 2-1).

- The consensus means and ranges for $\Delta^{8}$-THC are based on quantitative data from 18 laboratories (Figure 2-9), 23 laboratories (Figure 2-10), and 3 laboratories (Figure 2-11) for Hemp Oil 1, Hemp Oil 2, and Hemp Oil 2a, respectively. Data from participants submitting only one measurement were included in Table 2-4 but were not included in the calculation of consensus statistics. ${ }^{2}$

- A comparison of individual laboratory means for $\Delta^{8}$-THC in Hemp Oil 1 and Hemp Oil 2 is summarized in Figure 2-12 for laboratories who reported results for both samples

\section{Total THC}

- The mass fractions (\%) for total THC in the hemp oil samples were determined by NIST using LC-PDA as described in Section 1 and are summarized in Table 2-1. These NIST values are used as the target means and ranges summarized in Table 2-5 for comparison to the participant results.

- The target and consensus means and ranges are summarized for total THC via different analytical methods in Figure 2-13, Figure 2-14, and Figure 2-15, which include data from 
laboratories submitting two or three measurements for total THC. Data from participants submitting only one measurement were included in Table 2-5 but were not included in the calculation of consensus statistics. ${ }^{2}$

- For total THC in Hemp Oil 1, the consensus range was based on quantitative results from 52 laboratories and overlaps approximately $70 \%$ of the target range (Figure 2-13).

- The individual laboratory means or thresholds from 32 laboratories $(62 \%$ of those reporting results) were outside the NIST range of tolerance for total THC in Hemp Oil 1.

- The individual laboratory means from 7 laboratories (13\% of those reporting results) were outside the acceptable $Z_{\text {comm }}^{\prime}$ score for total THC in Hemp Oil 1.

- The threshold or LOQ for 1 of 1 laboratory reporting a qualitative result was below the target mean for total THC in Hemp Oil 1.

- For total THC in Hemp Oil 2, the consensus range was based on quantitative results from 57 laboratories and overlaps approximately $50 \%$ of the target range (Figure 2-14).

- The individual laboratory means or thresholds from 34 laboratories $(60 \%$ of those reporting results) were outside the NIST range of tolerance for total THC in Hemp Oil 2.

- The individual laboratory means from 6 laboratories (11\% of those reporting results) were outside the acceptable $Z_{\text {comm }}^{\prime}$ score for total THC in Hemp Oil 2.

- The thresholds or LOQs for 2 of 2 laboratories reporting a qualitative result were below the target mean for total THC in Hemp Oil 2.

- For total THC in Hemp Oil 2a, the consensus range was based on quantitative results from 11 laboratories and overlaps approximately $75 \%$ of the target range (Figure 2-15).

- The individual laboratory means or thresholds from 8 laboratories $(73 \%$ of those reporting results) were outside the NIST range of tolerance for total THC in Hemp Oil 2a.

- No individual laboratory means were outside the acceptable $Z_{\text {comm }}^{\prime}$ score for total THC in Hemp Oil 2a.

- No results were reported using thresholds or LOQs for total THC in Hemp Oil 2a.

- A comparison of individual laboratory means for total THC in Hemp Oil 1 and Hemp Oil 2 is summarized in Figure 2-16 for laboratories who reported results for both samples.

Overall

- The between-laboratory variabilities for determination of $\Delta^{9}$-THC, THCA, $\Delta^{8}$-THC, and total THC in the hemp oil samples are shown in the table below.

Between-Laboratory Variability (\% RSD)

\begin{tabular}{|c|c|c|c|}
\hline Analyte & Hemp Oil 1 & Hemp Oil 2 & Hemp Oil 2a \\
\hline$\Delta^{9}$-THC & 2.2 & 2.7 & 6.4 \\
\hline THCA & 28.8 & 27.9 & 85.1 \\
\hline$\Delta^{8}-\mathrm{THC}$ & 28.4 & 29.2 & 57.2 \\
\hline Total THC & 2.8 & 3.4 & 11.9 \\
\hline
\end{tabular}


Study Discussion and Technical Recommendations

The following recommendations are based on results obtained from the participants in this study.

$\Delta^{9}-T H C$

- Approximately $17 \%$ of the laboratories reporting results for $\Delta^{9}-\mathrm{THC}$ provided values outside the consensus ranges for both Hemp Oil 1 and Hemp Oil 2 (Figure 2-4).

- Laboratories reporting results below the consensus mean in Hemp Oil 1 also reported results below the consensus mean for Hemp Oil 2. The reverse is also observed for laboratories reporting values above the consensus mean in both samples. Trends of this type often indicate a calibration bias.

- The between-laboratory variability for $\Delta^{9}$-THC was higher in Hemp Oil 2a (6.4\%) than Hemp Oil $1(2.2 \%)$ or Hemp Oil $2(2.7 \%)$. The variability of individual laboratory means was lower for $\Delta^{9}$-THC in Hemp Oil 1 (4.6\%) and Hemp Oil 2 (4.1\%) in comparison to Hemp Oil 2a $(8.3 \%)$.

- Hemp Oil 2a was prepared through a methanol/ethanol extraction of Hemp Oil 2 at NIST. The additional processing of Hemp Oil $2 \mathrm{a}$ was expected to result in minimal variability both within and among participating laboratories.

- The elevated level of variability may be an artifact of significantly fewer laboratories reporting results for Hemp Oil 2a (11) compared to Hemp Oil 1 (65) and Hemp Oil 2 (72).

- No additional trends were observed for the sample preparation, analytical methods, and/or analytical parameters reported for $\Delta^{9}$-THC in the three hemp oil samples.

THCA

- Approximately $27 \%$ of the laboratories reporting results for THCA provided values outside the consensus ranges for both Hemp Oil 1 and Hemp Oil 2 (Figure 2-8).

- Laboratories reporting results above the consensus mean in Hemp Oil 1 did not necessarily report results above the consensus mean for Hemp Oil 2. Trends of this type often represent potential sample interferences and miss identifications due to levels of THCA being at or below participants LOQs.

- Most laboratories reported that THCA was present in the samples at or below their LOQ (nonzero values). The low levels resulted in large consensus ranges and between-laboratory variabilities $(28 \%$ to $85 \%)$.

- Approximately $5 \%$ of the laboratories reporting results used LC-MS or LC-MS/MS methods with most having adequate LOQs to determine THCA at the consensus levels in Hemp Oil 1, Hemp Oil 2, and Hemp Oil 2a.

- Approximately $93 \%$ of the laboratories reporting results used LC-UV or LC-PDA methods with only $17 \%, 22 \%$, and $12 \%$ of these laboratories with sufficient LOQs to determine THCA at the consensus levels in Hemp Oil 1, Hemp Oil 2, and Hemp Oil 2a, respectively.

- No additional trends were observed for the sample preparation, analytical methods, and/or analytical parameters reported for THCA in the three hemp oil samples.

$\Delta^{8}-T H C$

- Approximately $18 \%$ of the laboratories reporting results for $\Delta^{8}$-THC provided values outside the consensus range for both Hemp Oil 1 and Hemp Oil 2 (Figure 2-12). 
- Laboratories reporting results above the consensus mean in Hemp Oil 1 also reported results above the consensus mean for Hemp Oil 2. Trends of this type often indicate a calibration bias.

- Most laboratories reported that $\Delta^{8}$-THC was present in the samples at or below their LOQ (non-zero values), resulting in large consensus ranges and between-laboratory variabilities (28 $\%$ to $57 \%$ ).

- Approximately $6 \%$ of the laboratories reporting results used LC-MS or LC-MS/MS methods with only 1 laboratory having sufficient LOQs to determine $\Delta^{8}$-THC at the consensus levels in Hemp Oil 1, Hemp Oil 2, and Hemp Oil 2a.

- Approximately $91 \%$ of the laboratories reporting results used LC-UV or LC-PDA methods with only $29 \%, 30 \%$, and $33 \%$ of these laboratories with adequate LOQs to determine $\Delta^{8}$-THC at the consensus levels in Hemp Oil 1, Hemp Oil 2, and Hemp Oil 2a, respectively.

- No additional trends were observed for the sample preparation, analytical methods, and/or analytical parameters reported for $\Delta^{8}$-THC in the three hemp oil samples.

\section{Total THC}

- Approximately $14 \%$ of the laboratories reporting results for total THC provided values outside the consensus range for both Hemp Oil 1 and Hemp Oil 2 (Figure 2-16).

- Laboratories reporting results below the consensus mean in Hemp Oil 1 also reported results below the consensus mean for Hemp Oil 2. The reverse is also observed for laboratories reporting values above the consensus mean in both samples. Trends of this type often indicate a calibration bias.

- The between-laboratory variability for total THC was higher in Hemp Oil 2a (11.9\%) than Hemp Oil 1 (2.8 \%) or Hemp Oil 2 (3.4\%). The variability of individual laboratory means was lower for total THC in Hemp Oil 1 (4.8 \%) and Hemp Oil 2 (4.0 \%) in comparison to Hemp Oil 2a $(8.1 \%)$.

- Hemp Oil 2a was prepared through a methanol/ethanol extraction of Hemp Oil 2 at NIST. The additional processing of Hemp Oil 2a was expected to result in minimal variability both within and among participating laboratories.

- The elevated level of variability may be an artifact of significantly fewer laboratories reporting results for Hemp Oil 2a (11) compared to Hemp Oil 1 (52) and Hemp Oil 2 (57).

- No additional trends were observed for the sample preparation, analytical methods, and/or analytical parameters reported for total THC in the three hemp oil samples.

Overall

- Proper storage conditions are important for hemp oils because THCA can readily convert to $\Delta^{9}$-THC when store at elevated or room temperatures. ${ }^{4}$

- Participants were asked to store the samples under controlled refrigeration $\left(\approx 4{ }^{\circ} \mathrm{C}\right)$.

- Laboratories should perform in-house evaluations of their storage conditions through stability test of their hemp oils at various environmental factors such as temperature, humidity, and light exposure. ${ }^{5}$

\footnotetext{
${ }^{5}$ ASTM INTERNATIONAL, ASTM D8309; Standard Guide for Stability Testing of Cannabis-Based Products, 2021.
} 
- Laboratories should make total THC determinations via experimental conversion of THCA to $\Delta^{9}$-THC (using elevated temperature or specific chemical reagents) or via calculation of total THC from the sum of measured $\Delta^{9}$-THC and THCA in the sample using the equation below.

$$
\text { Total THC }=\text { mass } \% \Delta^{9}-T H C+(0.877 \times \text { mass } \% \text { THCA })
$$

- Over 100 cannabinoids have been identified in Cannabis plant samples with similarities in structure and molecular mass to $\Delta^{9}$-THC. As a result, chromatographic peak identity should always be confirmed using appropriate reference spectra of pure standards for cannabinoids and is particularly important for cannabinoids that are present at levels close to the method LOQ. Analytical methods should be able to clearly distinguish between cannabinoids.

- The use of appropriate calibration materials and quality assurance samples to establish that a method is in control and being performed correctly may reduce the likelihood of outlying data. Quality assurance samples can be commercially available reference materials (CRMs, SRMs, or RMs) or materials prepared in-house.

- Measurement results should be reported accurately.

- Reported values should be the mass fraction (\%) of the $\Delta^{9}$-THC, THCA, and total THC in the three hemp oil samples. Participants who reported values based on a volume fraction (\%) for Hemp Oil 2 or Hemp Oil 2a should use the density of the sample for the conversion to mass fraction. Hemp Oil 1 was too viscous to prepare dilutions by volume.

- Zero is not a quantity that can be measured. If values are below LOQ, results should be reported as such. A more appropriate result would be to report that a value is below the LOQ (e.g., " $<0.02 \%$ ").

- Laboratories reporting results based on a certain threshold should enter the numerical threshold (e.g., " $<1$ ").

- Laboratories reporting results flagged as outliers should check for calculation errors when preliminary data tables are sent for inspection. One example is to confirm that factors for all dilutions have been properly tabulated or that results are reported in the requested units. 
Table 2-1. Individualized data summary table (NIST) for $\Delta^{9}$-THC, THCA, $\Delta^{8}$-THC, and total THC in hemp oils.

\section{National Institute of Standards and Technology}

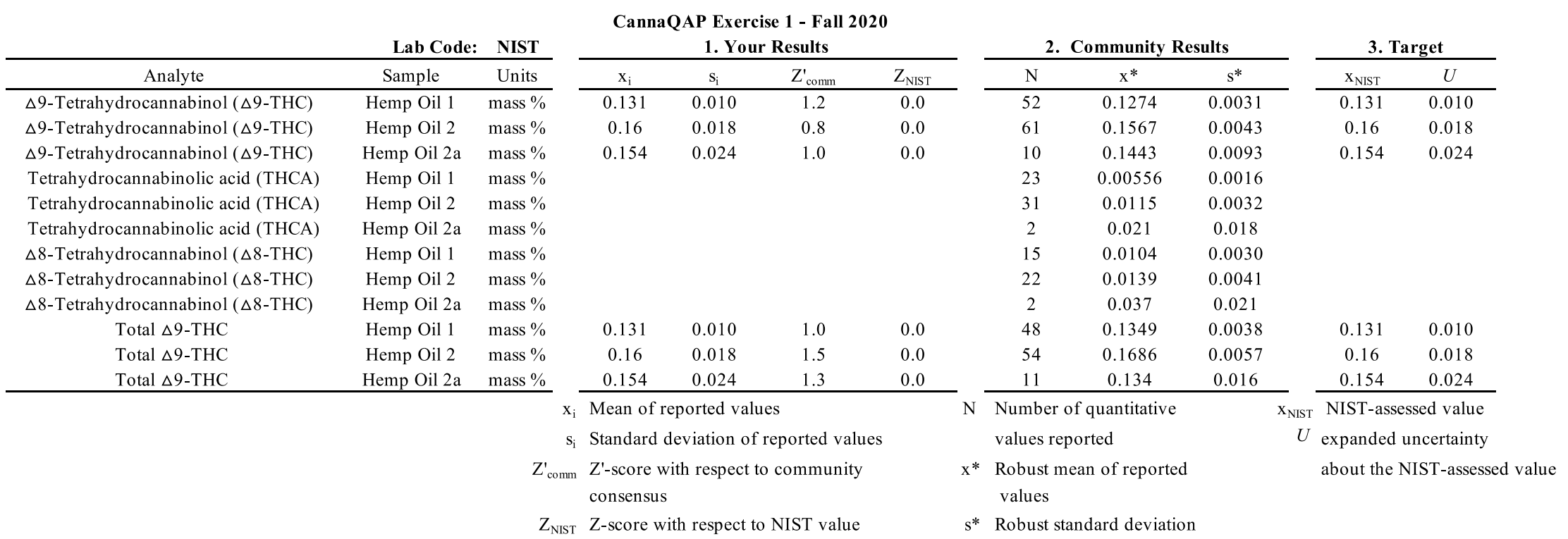


Table 2-2. Data summary table for $\Delta^{9}$-THC in hemp oils. Data highlighted in red have been flagged as a data entry of zero or results that include text (e.g., "< LOQ" or "present"). Data highlighted in blue have been identified as outside the consensus tolerance limits and would be estimated to result in an unacceptable $Z_{\text {comm }}^{\prime}$ score, $\left|Z_{\text {comm }}^{\prime}\right| \geq 2$. Note: This table spans two pages; the NIST values and consensus values are included on both pages for convenience.

\begin{tabular}{|c|c|c|c|c|c|c|c|c|c|c|c|c|c|c|c|c|}
\hline & \multirow[b]{3}{*}{ Lab } & \multicolumn{15}{|c|}{$\Delta 9$-Tetrahydrocannabinol $(\triangle 9-\mathrm{THC})$} \\
\hline & & \multicolumn{5}{|c|}{ Hemp Oil 1 (mass \%) } & \multicolumn{5}{|c|}{ Hemp Oil 2 (mass \%) } & \multicolumn{5}{|c|}{ Hemp Oil 2a (mass \%) } \\
\hline & & $\mathbf{A}$ & $\mathbf{B}$ & $\mathbf{C}$ & Avg & SD & $\mathbf{A}$ & $\mathbf{B}$ & $\mathbf{C}$ & Avg & SD & $\mathbf{A}$ & $\mathbf{B}$ & $\mathbf{C}$ & Avg & SD \\
\hline \multirow{55}{*}{ 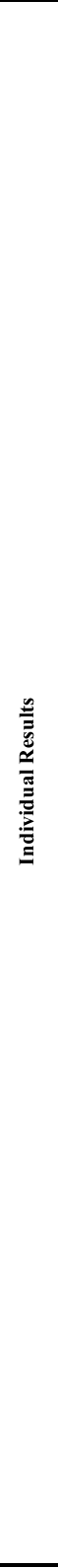 } & NIST & & & & 0.131 & 0.010 & & & & 0.160 & 0.018 & & & & 0.154 & 0.024 \\
\hline & A001 & 0.14 & 0.13 & 0.12 & 0.1300 & 0.0100 & 0.17 & 0.18 & 0.17 & 0.1733 & 0.0058 & & & & & \\
\hline & $\mathrm{A} 002$ & 0.13898 & 0.1326 & 0.12578 & 0.1325 & 0.0066 & 0.170171 & 0.171068 & 0.156471 & 0.1659 & 0.0082 & & & & & \\
\hline & A003 & & & & & & 0.174 & 0.163 & 0.157 & 0.1647 & 0.0086 & & & & & \\
\hline & $\mathrm{A} 004$ & 0.11 & 0.11 & 0.11 & 0.1100 & 0.0000 & 0.15 & 0.13 & 0.13 & 0.1367 & 0.0115 & & & & & \\
\hline & A005 & 0.14 & 0.125 & 0.135 & 0.1333 & 0.0076 & 0.17 & 0.169 & 0.172 & 0.1703 & 0.0015 & & & & & \\
\hline & A006 & 0.15 & & & 0.1500 & & 0.17 & & & 0.1700 & & & & & & \\
\hline & A007 & & & & & & 0.03 & 0.05 & 0.02 & 0.0333 & 0.0153 & & & & & \\
\hline & A008 & 0.069 & & & 0.0690 & & & & & & & & & & & \\
\hline & A009 & & & & & & & & & & & 0.164 & 0.164 & 0.176 & 0.1680 & 0.0069 \\
\hline & A010 & & & & & & & & & & & present & present & present & & \\
\hline & A011 & & & & & & & & & & & & & & & \\
\hline & A012 & & & & & & 0.14262 & 0.14309 & 0.14348 & 0.1431 & 0.0004 & & & & & \\
\hline & A013 & 0.134 & 0.141 & 0.144 & 0.1397 & 0.0051 & 0.151 & 0.147 & 0.145 & 0.1477 & 0.0031 & & & & & \\
\hline & A014 & 0.13 & & & 0.1300 & & 0.15 & & & 0.1500 & & & & & & \\
\hline & A015 & & & & & & & & & & & 0.18 & 0.12 & 0.28 & 0.1933 & 0.0808 \\
\hline & A016 & & & & & & & & & & & & & & & \\
\hline & A017 & 0.15 & & & 0.1500 & & 0.21 & & & 0.2100 & & & & & & \\
\hline & A018 & & & & & & & & & & & & & & & \\
\hline & A019 & 0.13 & & & 0.1300 & & 0.17 & & & 0.1700 & & & & & & \\
\hline & A 020 & 0.12328 & 0.124478 & 0.123915 & 0.1239 & 0.0006 & 0.144388 & 0.146124 & 0.160594 & 0.1504 & 0.0089 & & & & & \\
\hline & $\mathrm{A} 021$ & & & & & & & & & & & 0.05 & 0.08 & 0.07 & 0.0667 & 0.0153 \\
\hline & $\mathrm{A} 022$ & & & & & & & & & & & 0.1199 & 0.1218 & 0.1226 & 0.1214 & 0.0014 \\
\hline & A 023 & & & & & & 0.1835 & 0.1795 & 0.1822 & 0.1817 & 0.0020 & & & & & \\
\hline & A024 & & & & & & & & & & & 0.17 & 0.181 & 0.176 & 0.1757 & 0.0055 \\
\hline & A025 & & & & & & 0.15 & 0.11 & 0.16 & 0.1400 & 0.0265 & & & & & \\
\hline & A026 & $<0.15$ & $<0.15$ & $<0.15$ & $<0.15$ & & $<0.15$ & $<0.15$ & $<0.15$ & $<0.15$ & & & & & & \\
\hline & A027 & & & & & & & & & & & 0.156 & 0.158 & 0.158 & 0.1573 & 0.0012 \\
\hline & A 028 & 0.1538 & 0.1718 & 0.1537 & 0.1598 & 0.0104 & 0.2198 & 0.1871 & 0.2153 & 0.2074 & 0.0177 & & & & & \\
\hline & A029 & & & & & & $<2$ & $<2$ & $<2$ & $<2$ & & & & & & \\
\hline & A 030 & 0.186 & & & 0.1860 & & 0.182 & & & 0.1820 & & & & & & \\
\hline & A031 & 0.12 & 0.12 & 0.12 & 0.1200 & 0.0000 & 0.13 & 0.14 & 0.14 & 0.1367 & 0.0058 & & & & & \\
\hline & $\mathrm{A} 032$ & & & & & & & & & & & & & & & \\
\hline & A033 & 0.12 & 0.11 & 0.12 & 0.1167 & 0.0058 & 0.14 & 0.15 & 0.13 & 0.1400 & 0.0100 & & & & & \\
\hline & A034 & & & & & & 0.136 & 0.135 & 0.135 & 0.1353 & 0.0006 & & & & & \\
\hline & A 035 & 0.129 & & & 0.1290 & & 0.171 & 0.174 & 0.173 & 0.1727 & 0.0015 & & & & & \\
\hline & A036 & 0.135 & 0.137 & 0.14 & 0.1373 & 0.0025 & 0.181 & 0.182 & 0.187 & 0.1833 & 0.0032 & & & & & \\
\hline & A037 & 0.13 & 0.131 & 0.128 & 0.1297 & 0.0015 & 0.162 & 0.162 & 0.159 & 0.1610 & 0.0017 & & & & & \\
\hline & A 038 & 0.161 & 0.16 & 0.158 & 0.1597 & 0.0015 & 0.207 & 0.192 & 0.208 & 0.2023 & 0.0090 & & & & & \\
\hline & A039 & 0.09 & 0.07 & 0.08 & 0.0800 & 0.0100 & 0.1 & 0.1 & 0.09 & 0.0967 & 0.0058 & & & & & \\
\hline & A 040 & & & & & & & & & & & 0.12641 & 0.12562 & 0.12734 & 0.1265 & 0.0009 \\
\hline & A041 & 0.1 & 0.1 & 0.1 & 0.1000 & 0.0000 & 0.1 & 0.1 & 0.1 & 0.1000 & 0.0000 & & & & & \\
\hline & A042 & BLQ & BLQ & BLQ & & & BLQ & BLQ & BLQ & & & & & & & \\
\hline & A043 & 0.2 & 0.19 & 0.199 & 0.1963 & 0.0055 & 0.306 & 0.309 & 0.31 & 0.3083 & 0.0021 & & & & & \\
\hline & A044 & & & & & & & & & & & & & & & \\
\hline & A045 & & & & & & & & & & & & & & & \\
\hline & A046 & 0 & 0.1 & 0 & 0.0333 & 0.0577 & 0 & 0 & 0 & 0.0000 & 0.0000 & & & & & \\
\hline & A047 & 0.01 & 0.01 & 0.01 & 0.0100 & 0.0000 & 0.02 & 0.02 & 0.02 & 0.0200 & 0.0000 & & & & & \\
\hline & A048 & 0.067 & 0.068 & 0.088 & 0.0743 & 0.0118 & 0.082 & 0.08 & 0.08 & 0.0807 & 0.0012 & & & & & \\
\hline & A049 & 0.104 & & & 0.1040 & & 0.13 & & & 0.1300 & & & & & & \\
\hline & $\mathrm{A} 050$ & 0.134 & 0.135 & 0.134 & 0.1343 & 0.0006 & 0.096 & 0.093 & 0.0946 & 0.0945 & 0.0015 & & & & & \\
\hline & A051 & & & & & & & & & & & & & & & \\
\hline & A052 & & & & & & & & & & & & & & & \\
\hline & A053 & & & & & & & & & & & & & & & \\
\hline & $\mathrm{A} 055$ & 0.1315 & 0.1315 & 0.1348 & 0.1326 & 0.0019 & 0.1662 & 0.1602 & 0.161 & 0.1625 & 0.0033 & & & & & \\
\hline 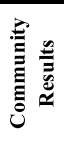 & & $\begin{array}{l}\text { Consensu } \\
\text { Consensu } \\
\text { Maximum } \\
\text { Minimum } \\
\mathrm{N}\end{array}$ & $\begin{array}{l}\text { Mean } \\
\text { Standard }\end{array}$ & Deviation & $\begin{array}{c}0.1276 \\
0.0029 \\
0.6600 \\
0.0000 \\
52\end{array}$ & & $\begin{array}{l}\text { Consensu } \\
\text { Consensu } \\
\text { Maximum } \\
\text { Minimum } \\
\mathrm{N}\end{array}$ & $\begin{array}{l}\text { s Mean } \\
\text { s Standard I }\end{array}$ & Deviation & $\begin{array}{c}0.1567 \\
0.0043 \\
0.7900 \\
0.0000 \\
61\end{array}$ & & $\begin{array}{l}\text { Consensu } \\
\text { Consensu } \\
\text { Maximum } \\
\text { Minimum } \\
\text { N }\end{array}$ & $\begin{array}{l}\text { Mean } \\
\text { Standard }\end{array}$ & Deviation & $\begin{array}{c}0.1443 \\
0.0093 \\
0.1933 \\
0.0667 \\
10\end{array}$ & \\
\hline
\end{tabular}




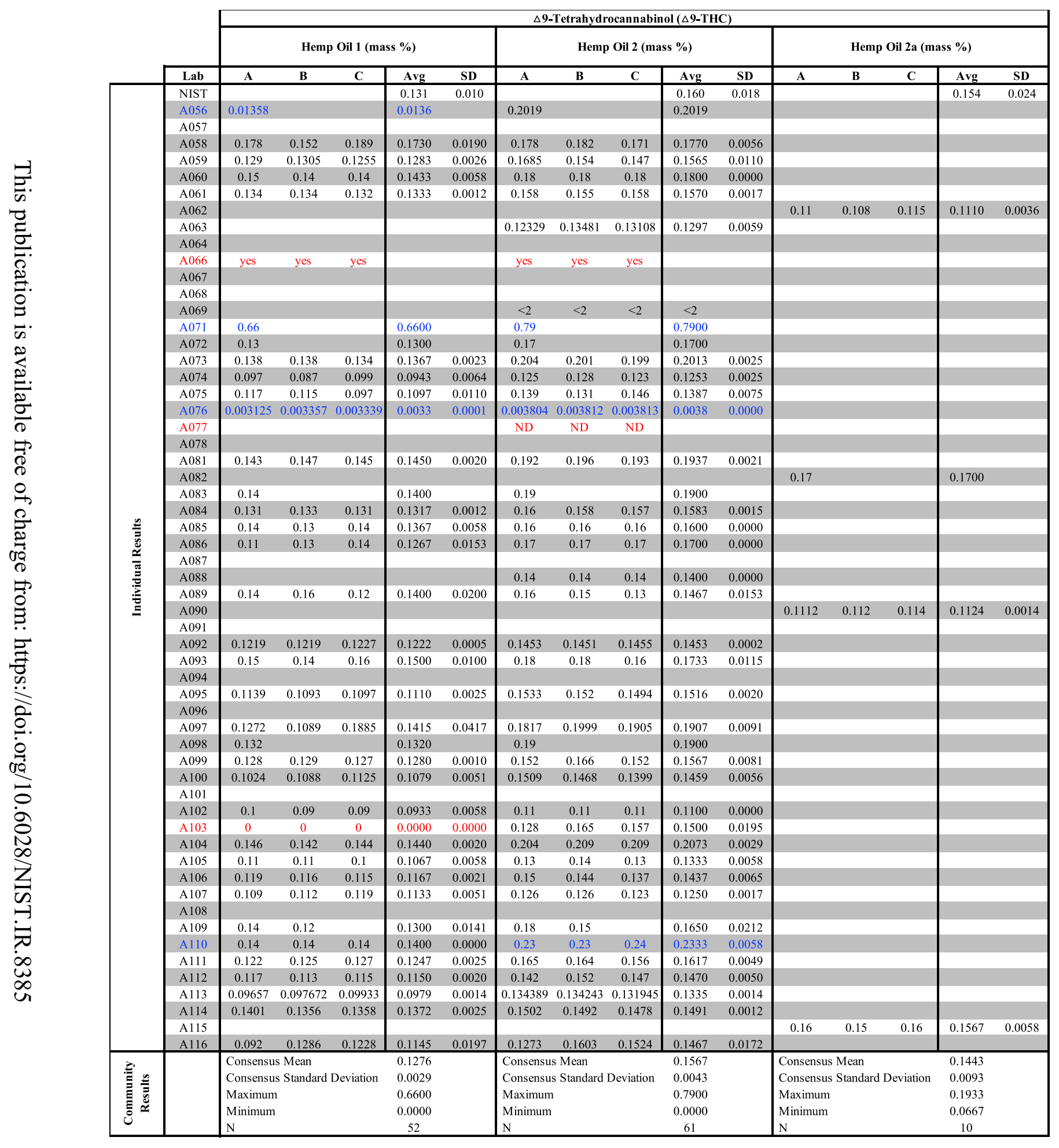




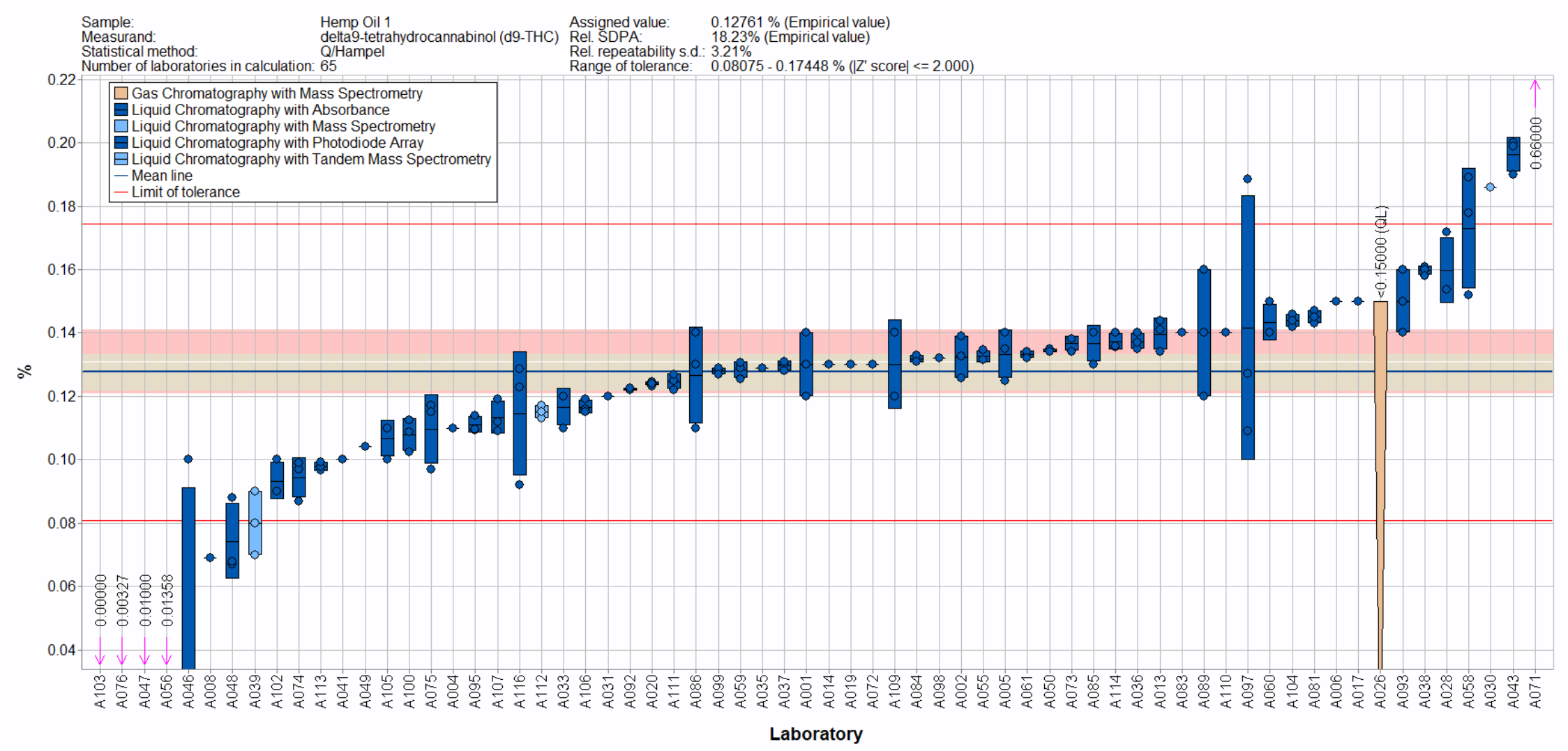

Figure 2-1. $\Delta^{9}$-THC in Hemp Oil 1 (data summary view - analytical method). In this view, individual laboratory data are plotted (circles) with the individual laboratory standard deviation (rectangle). The color of the data point represents the analytical method employed. The solid blue line represents the consensus mean, and the green shaded region represents the $95 \%$ confidence interval for the consensus mean. The solid red lines represent the consensus range of tolerance, calculated as the values above and below the consensus mean that result in an acceptable $Z_{\text {comm }}^{\prime}$ score, $\left|Z_{\text {comm }}^{\prime}\right| \leq 2$. The red shaded region represents the NIST range of tolerance, which encompasses the target value bounded by twice its uncertainty ( $U_{\text {NIST }}$ ) and represents the range that results in an acceptable $Z_{\text {NIST }}$ score, $\left|Z_{\text {NIST }}\right| \leq 2$. The shaded beige region represents the overlapping of the $95 \%$ confidence interval for the consensus mean (green region) and the NIST range of tolerance (red region). The downward triangle represents data reported as a threshold or LOQ value. 


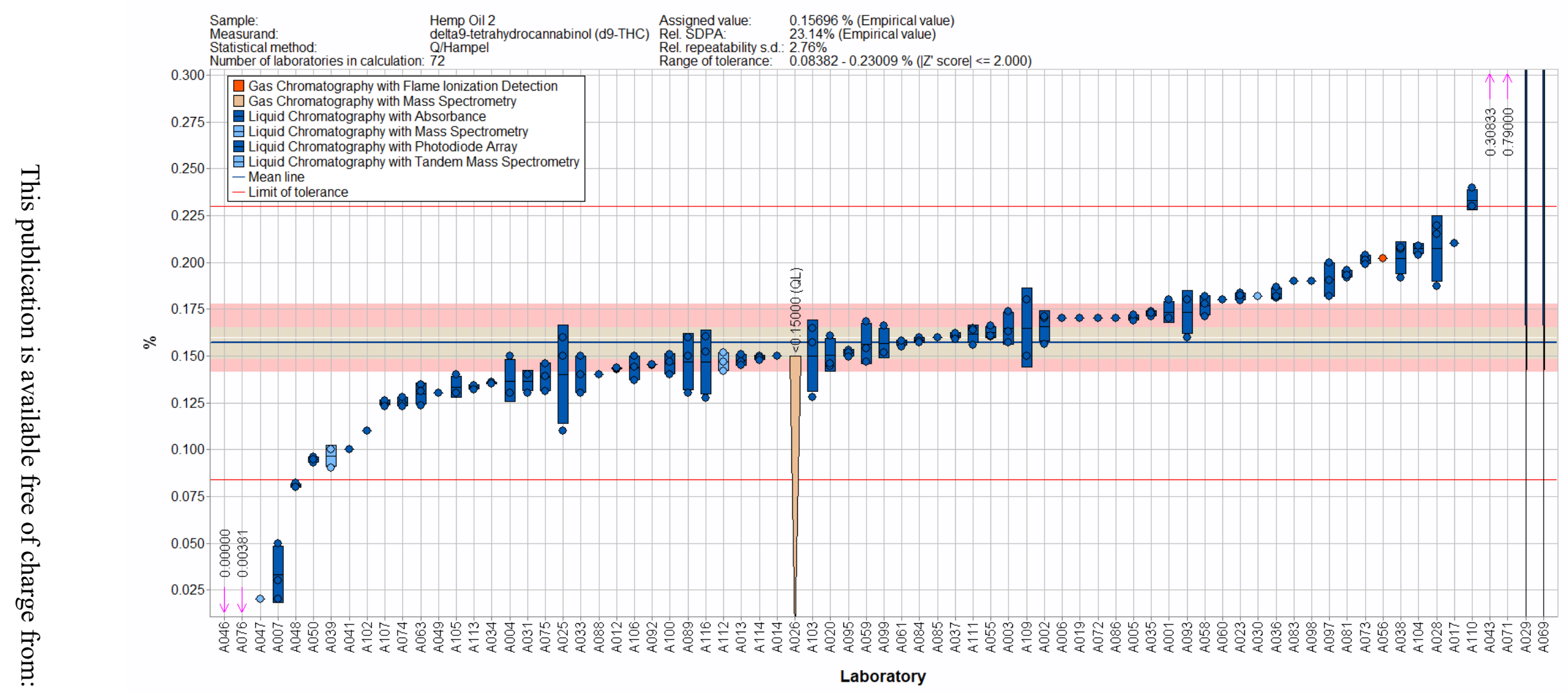

Figure 2-2. $\Delta^{9}$-THC in Hemp Oil 2 (data summary view - analytical method). In this view, individual laboratory data are plotted (circles) with the individual laboratory standard deviation (rectangle). The color of the data point represents the analytical method employed. The solid blue line represents the consensus mean, and the green shaded region represents the $95 \%$ confidence interval for the consensus mean. The solid red lines represent the consensus range of tolerance, calculated as the values above and below the consensus mean that result in an acceptable $Z_{\text {comm }}^{\prime}$ score, $\left|Z_{\text {comm }}^{\prime}\right| \leq 2$. The red shaded region represents the NIST range of tolerance, which encompasses the target value bounded by twice its uncertainty ( $U_{\text {NIST }}$ ) and represents the range that results in an acceptable $Z_{\text {NIST }}$ score, $\left|Z_{\text {NIST }}\right| \leq 2$. The shaded beige region represents the overlapping of the $95 \%$ confidence interval for the consensus mean (green region) and the NIST range of tolerance (red region). The downward triangle represents data reported as a threshold or LOQ value. 


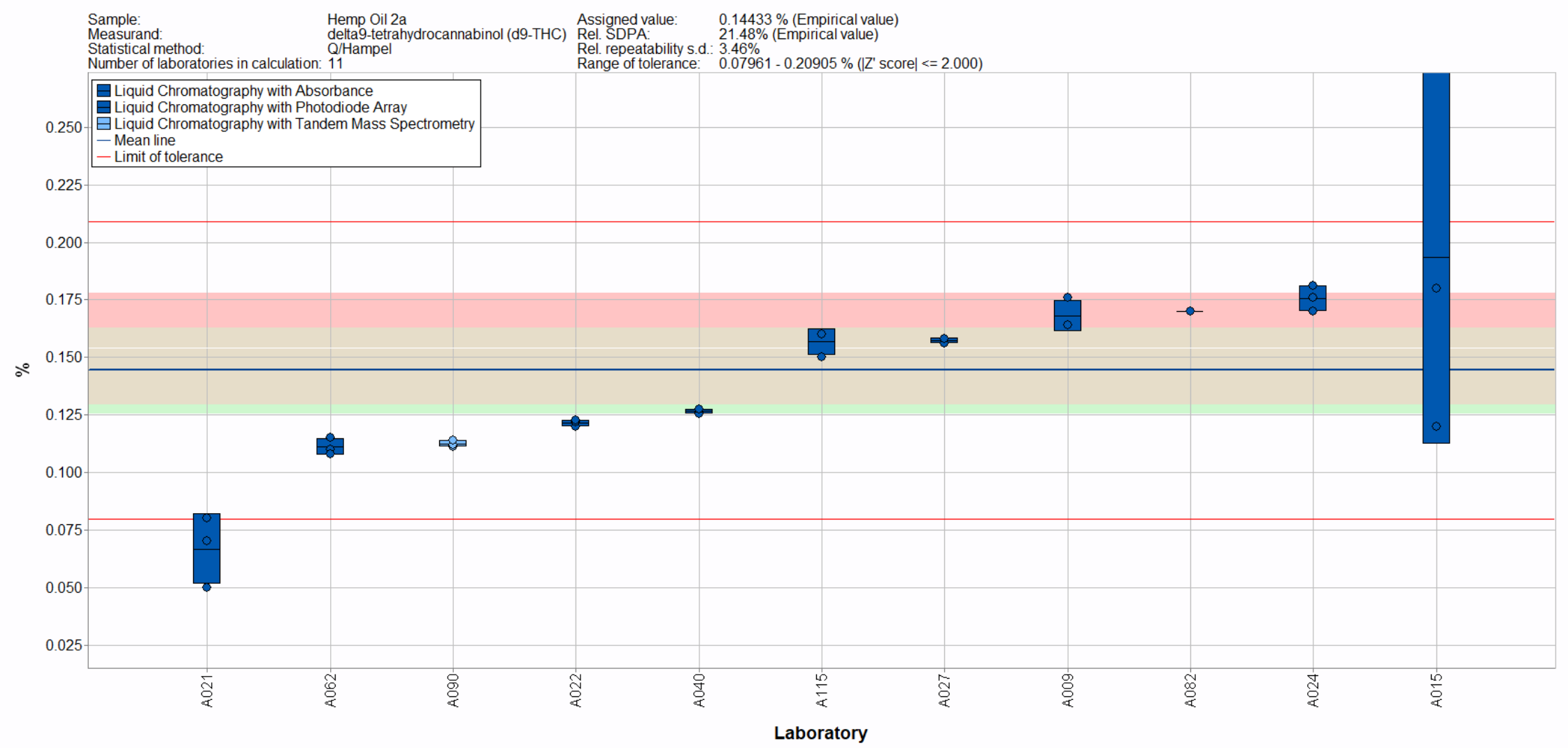

Figure 2-3. $\Delta^{9}$-THC in Hemp Oil 2a (data summary view - analytical method). In this view, individual laboratory data are plotted (circles) with the individual laboratory standard deviation (rectangle). The color of the data point represents the analytical method employed. The solid blue line represents the consensus mean, and the green shaded region represents the $95 \%$ confidence interval for the consensus mean. The solid red lines represent the consensus range of tolerance, calculated as the values above and below the consensus mean that result in an acceptable $Z_{\text {comm }}^{\prime}$ score, $\left|Z_{\text {comm }}^{\prime}\right| \leq 2$. The red shaded region represents the NIST range of tolerance, which encompasses the target value bounded by twice its uncertainty $\left(U_{\mathrm{NIST}}\right)$ and represents the range that results in an acceptable $Z_{\mathrm{NIST}}$ score, $\left|Z_{\mathrm{NIST}}\right| \leq 2$. The shaded beige region represents the overlapping of the $95 \%$ confidence interval for the consensus mean (green region) and the NIST range of tolerance (red region). 


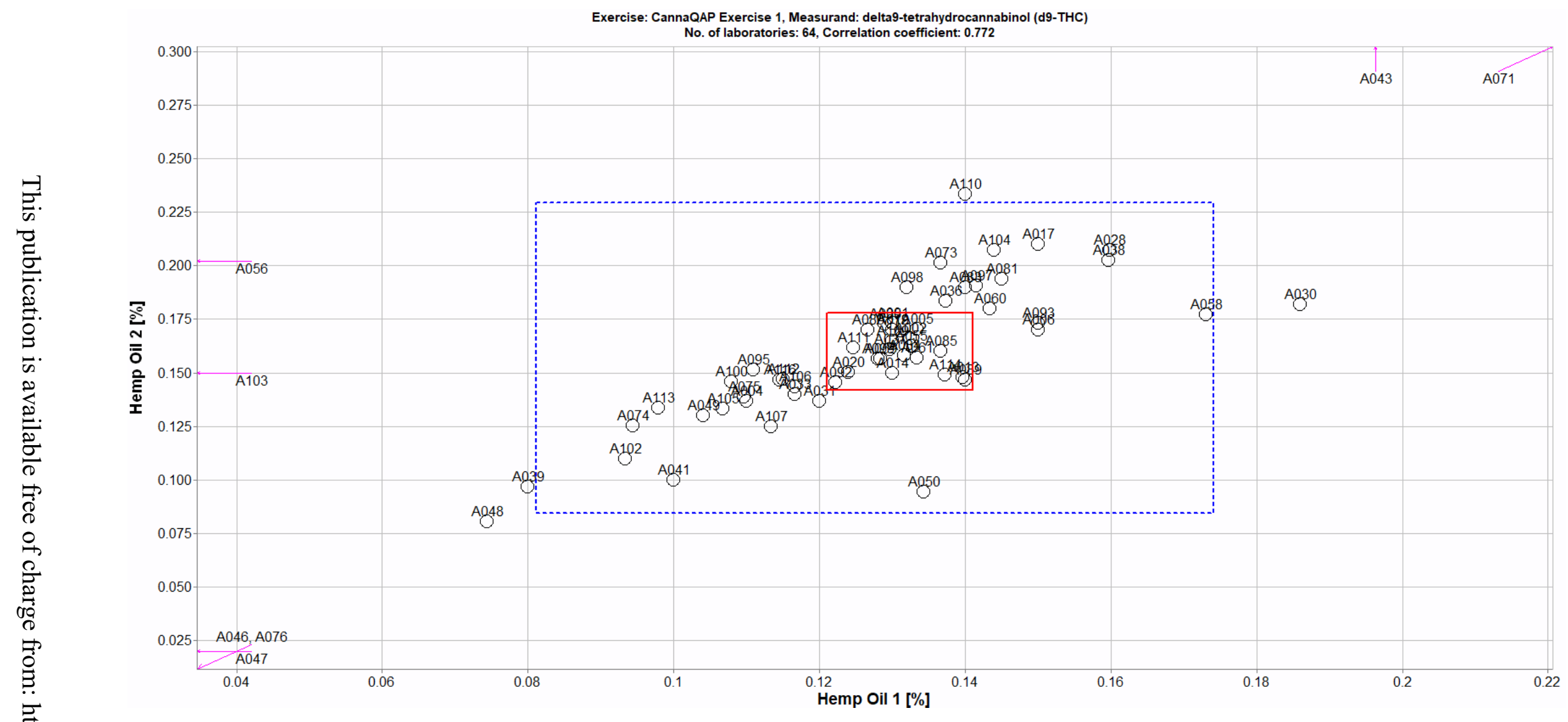

Figure 2-4. Laboratory means for $\Delta^{9}$-THC in Hemp Oil 1 and Hemp Oil 2 (sample/sample comparison view). In this view, the individual laboratory mean for one sample (Hemp Oil 1) is compared to the individual laboratory mean for a second sample (Hemp Oil 2). The solid red box represents the NIST range of tolerance for the two samples, Hemp Oil 1 (x-axis) and Hemp Oil 2 (y-axis), which encompasses the target values bounded by their uncertainties $\left(U_{\mathrm{NIST}}\right)$ and represents the range that results in an acceptable $Z_{\mathrm{NIST}}$ score, $\left|Z_{\mathrm{NIST}}\right| \leq 2$. The dotted blue box represents the consensus range of tolerance for Hemp Oil 1 (x-axis) and Hemp Oil 2 (y-axis), calculated as the values above and below the consensus means that result in an acceptable $Z_{\text {comm }}^{\prime}$ score, $\left|Z_{\text {comm }}^{\prime}\right| \leq 2$. 
Table 2-3. Data summary table for THCA in hemp oils. Data highlighted in red have been flagged as a data entry of zero or results that include text (e.g., "<LOQ" or "present"). Data highlighted in blue have been identified as outside the consensus tolerance limits and would be estimated to result in an unacceptable $Z_{\text {comm }}^{\prime}$ score, $\left|Z_{\text {comm }}^{\prime}\right| \geq 2$. Note: This table spans two pages; the NIST values and consensus values are included on both pages for convenience.

\begin{tabular}{|c|c|c|c|c|c|c|c|c|c|c|c|c|c|c|c|c|}
\hline & \multirow[b]{3}{*}{ Lab } & \multicolumn{15}{|c|}{ Tetrahydrocannabinolic acid (THCA) } \\
\hline & & \multicolumn{5}{|c|}{ Heap Oill (mass \%) } & \multicolumn{5}{|c|}{ Heap OAl 2 (mass \%) } & \multicolumn{5}{|c|}{ Henp OAl 2a (-2ass \%) } \\
\hline & & $\mathbf{A}$ & $\mathbf{B}$ & $\mathbf{C}$ & Avg & SD & $\mathbf{A}$ & $\mathbf{B}$ & $\mathbf{C}$ & Avg & SD & $\mathbf{A}$ & $\mathbf{B}$ & $\mathbf{C}$ & Avg & SD \\
\hline \multirow{55}{*}{ 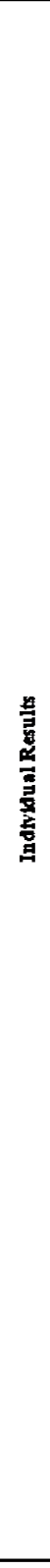 } & NIST & & & & & & & & & & & & & & & \\
\hline & A 001 & $<0.15$ & $<0.15$ & $<0.15$ & $<0.15$ & & $<0.15$ & $<0.15$ & $<0.15$ & $<0.15$ & & & & & & \\
\hline & A002 & $<0.0084$ & $<0.0084$ & $<0.0084$ & $<0.0084$ & & $<0.0084$ & $<0.0084$ & $<0.0084$ & $<0.0084$ & & & & & & \\
\hline & A 003 & & & & & & $<0.01$ & $<0.01$ & $<0.01$ & $<0.01$ & & & & & & \\
\hline & A004 & 0.11 & 008 & 0.07 & 00867 & 0.0208 & 0.19 & 0.19 & 0.14 & 0.1733 & 0.0289 & & & & & \\
\hline & A 005 & 0 & 0 & 0 & 0.0000 & 0.0000 & 0 & 0 & 0 & 0.0000 & 0.0000 & & & & & \\
\hline & A006 & 0.01 & & & 00100 & & 0.03 & & & 00300 & & & & & & \\
\hline & A 007 & & & & & & 0.12 & 0.13 & 0.13 & 0.1267 & 0.0058 & & & & & \\
\hline & A008 & & & & & & & & & & & & & & & \\
\hline & A 009 & & & & & & & & & & & $<0.00001$ & $<0.00001$ & $<0.00001$ & $<0.00001$ & \\
\hline & A010 & & & & & & & & & & & & & & & \\
\hline & A 011 & & & & & & & & & & & & & & & \\
\hline & A012 & & & & & & $\mathbf{0}$ & 0 & 0 & 00000 & 0.0000 & & & & & \\
\hline & A 013 & & & & & & & & & & & & & & & \\
\hline & A014 & $<0.09$ & & & $<0.09$ & & $<0.09$ & & & $<0.09$ & & & & & & \\
\hline & A 015 & & & & & & & & & & & & & & & \\
\hline & A016 & & & & & & & & & & & & & & & \\
\hline & A 017 & $<0.02$ & & & $<0.02$ & & $<0.02$ & & & $<0.02$ & & & & & & \\
\hline & A018 & & & & & & & & & & & & & & & \\
\hline & A 019 & $<0.09$ & & & $<0.09$ & & $<0.09$ & & & $<0.09$ & & & & & & \\
\hline & A020 & & & & & & 0.002196 & 0.002276 & 0.002097 & 0.0022 & 0.0001 & & & & & \\
\hline & A 021 & & & & & & & & & & & $<0.05$ & $<0.05$ & $<0.05$ & $<0.05$ & \\
\hline & A022 & & & & & & & & & & & & & & & \\
\hline & A 023 & & & & & & 0 & 0 & 0 & 0.0000 & 0.0000 & & & & & \\
\hline & A024 & & & & & & & & & & & $<0.010$ & 0.014 & 0.01 & 0.012 & 0.003 \\
\hline & A 025 & & & & & & & & & & & & & & & \\
\hline & A026 & & & & & & & & & & & & & & & \\
\hline & A 027 & & & & & & & & & & & $<0.0057$ & $<0.0057$ & $<0.0057$ & $\mid<0.0057$ & \\
\hline & A028 & $<0.0470$ & $<0.0470$ & $<0.0470$ & $<0.0470$ & & 0.0502 & 0.05 & 0.0491 & 0.0498 & 0.0006 & & & & & \\
\hline & A 029 & & & & & & $<2$ & $<2$ & $<2$ & $<2$ & & & & & & \\
\hline & A030 & & & & & & & & & & & & & & & \\
\hline & A 031 & $<0.05$ & $<0.05$ & $<0.05$ & $<0.05$ & & $<0.15$ & $<0.15$ & $<0.15$ & $<0.15$ & & & & & & \\
\hline & A032 & & & & & & & & & & & & & & & \\
\hline & A 033 & 0 & 0 & 0 & 0.0000 & 0.0000 & 0 & 0 & 0 & 0.0000 & 0.0000 & & & & & \\
\hline & A034 & & & & & & $<0.01$ & $<0.01$ & $<0.01$ & $<0.01$ & & & & & & \\
\hline & A 035 & $<0.00250$ & & & $<0.00250$ & & $<0.00250$ & $<0.00250$ & $<0.00250$ & $<0.00250$ & & & & & & \\
\hline & A036 & $<0206$ & $<0.206$ & $<0206$ & $<0206$ & & $<0247$ & $<0.247$ & $<0247$ & $<0247$ & & & & & & \\
\hline & A 037 & $<0.05$ & $<0.05$ & $<0.05$ & $<0.05$ & & $<0.05$ & $<0.05$ & $<0.05$ & $<0.05$ & & & & & & \\
\hline & A038 & $<0.025$ & $<0.025$ & $<0.025$ & $<0.025$ & & $<0.025$ & $<0.025$ & $<0.025$ & $<0.025$ & & & & & & \\
\hline & A 039 & 0 & 0 & 0 & 0.0000 & 0.0000 & 0 & 0 & 0 & 0.0000 & 0.0000 & & & & & \\
\hline & A040 & & & & & & & & & & & ND & ND & ND & & \\
\hline & A 041 & 0.1 & 0 & 0 & 0.0333 & 0.0577 & 0 & 0 & 0 & 0.0000 & 0.0000 & & & & & \\
\hline & A043 & 0.004 & 0.004 & 0.004 & 0.0040 & 0.0000 & 0.01 & 0.011 & 0.011 & 0.0107 & 0.0006 & & & & & \\
\hline & A 044 & & & & & & & & & & & & & & & \\
\hline & A045 & & & & & & & & & & & & & & & \\
\hline & A 046 & 0 & 0 & 0 & 0.0000 & 0.0000 & 0 & 0 & 0 & 0.0000 & 0.0000 & & & & & \\
\hline & A047 & 0 & 0 & 0 & 0.0000 & 0.0000 & 0 & 0 & 0 & 0.0000 & 0.0000 & & & & & \\
\hline & A 048 & 0.009 & 0.009 & 0.009 & 0.0090 & 0.0000 & 0.039 & 0.039 & 0.039 & 0.0390 & 0.0000 & & & & & \\
\hline & A049 & 0 & & & 0.0000 & & 0 & & & 00000 & & & & & & \\
\hline & A 050 & $<0.01$ & $<0.01$ & $<0.01$ & $<0.01$ & & $<0.01$ & $<0.01$ & $<0.01$ & $<0.01$ & & & & & & \\
\hline & A051 & & & & & & & & & & & & & & & \\
\hline & A 052 & & & & & & & & & & & & & & & \\
\hline & A053 & & & & & & & & & & & & & & & \\
\hline & A 054 & $<0.06$ & $<0.06$ & $<0.06$ & $<0.06$ & & $<0.06$ & $<0.06$ & $<0.06$ & $<0.06$ & & & & & & \\
\hline & A055 & 0 & 0 & 0 & 0.0000 & 0.0000 & 0 & 0 & 0 & 0.0000 & 0.0000 & & & & & \\
\hline 戛着 & & \begin{tabular}{|l} 
Consensus \\
Consensus \\
Maximum \\
Minimum \\
$\mathbf{N}$
\end{tabular} & $\begin{array}{l}\text { s Mean } \\
\text { s Standard } \\
\end{array}$ & Deviation & $\begin{array}{c}0.0056 \\
0.0016 \\
14767 \\
0.0000 \\
23\end{array}$ & & \begin{tabular}{|l} 
Consensu \\
Consensu \\
Maximum \\
Minimum \\
N
\end{tabular} & $\begin{array}{l}\text { s Mean } \\
s \text { Standard I } \\
\end{array}$ & Deviation & $\begin{array}{c}0.0115 \\
0.0032 \\
24367 \\
0.0000 \\
31\end{array}$ & & \begin{tabular}{|l} 
Consensus \\
Consensus \\
Maximum \\
Minimum \\
N
\end{tabular} & $\begin{array}{l}\text { s Mean } \\
\text { s Standand D } \\
\end{array}$ & Deviation & $\begin{array}{c}0.021 \\
0.018 \\
0.050 \\
0.000 \\
2\end{array}$ & \\
\hline
\end{tabular}




\begin{tabular}{|c|c|c|c|c|c|c|c|c|c|c|c|c|c|c|c|c|c|}
\hline & & & & & & & & Tet & mhydroca & mnabinolic & $\operatorname{acid}(\mathrm{THC}$ & & & & & & \\
\hline & & & & He=p & Oil 1 (mas & iss \%) & & & He=p & p OAl 2 (na & ss \%) & & & Henp & O12 (n) & ss \%) & \\
\hline & & Lab & $\mathbf{A}$ & B & $\mathbf{C}$ & Avg & SD & $\mathbf{A}$ & B & $\mathbf{C}$ & Avg & SD & $\mathbf{A}$ & B & $\mathbf{C}$ & Avg & SD \\
\hline & & NIST & & & & & & & & & & & & & & & \\
\hline & & $\mathrm{A} 057$ & & & & & & & & & & & & & & & \\
\hline & & A058 & & & & & & & & & & & & & & & \\
\hline & & A 059 & 0 & 0 & 0 & 0.0000 & 0.0000 & 0 & 0 & 0 & 0.0000 & 0.0000 & & & & & \\
\hline & & $\mathbf{A} 060$ & $<0.02$ & $<0.02$ & $<0.02$ & $<0.02$ & & $<0.02$ & $<0.02$ & $<0.02$ & $<0.02$ & & & & & & \\
\hline$\square$ & & $\mathrm{A} 061$ & 0 & 0 & 0 & 0.0000 & 0.0000 & 0 & 0 & 0 & 0.0000 & 0.0000 & & & & & \\
\hline$\vec{\sim}$. & & A062 & & & & & & & & & & & $<0.01$ & $<0.01$ & $<0.01$ & $<0.01$ & \\
\hline$\nabla$ & & A 063 & & & & & & 0.06607 & 0.06634 & 0.06578 & 0.0661 & 0.0003 & & & & & \\
\hline 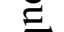 & & A064 & & & & & & & & & & & & & & & \\
\hline$\underline{\sigma}$ & & A 066 & & & & & & & & & & & & & & & \\
\hline$\vec{\Omega}$ & & A068 & & & & & & & & & & & & & & & \\
\hline$\stackrel{2}{\Rightarrow}$ & & A 069 & & & & & & $<2$ & $<2$ & $<2$ & $<2$ & & & & & & \\
\hline 0 & & $\mathrm{~A} 071$ & 0 & & & 0.0000 & & 0 & & & 0.0000 & & & & & & \\
\hline 追 & & A 072 & & & & & & & & & & & & & & & \\
\hline$\ddot{\infty}$. & & $\mathrm{A} 073$ & 0 & 0 & 0 & 0.0000 & 0.0000 & 0 & 0 & 0 & 0.0000 & 0.0000 & & & & & \\
\hline טم & & A 074 & $<0.009$ & $<0.009$ & $<0.009$ & $<0.009$ & & $<0.009$ & $<0.009$ & $<0.009$ & $<0.009$ & & & & & & \\
\hline$\underset{\Omega}{2}$ & & A 075 & 0.007 & 0.05 & 0.008 & 0.0217 & 0.0245 & 0.056 & 0.052 & 0.057 & 0.0550 & 0.0026 & & & & & \\
\hline ڤ. & & A 076 & 0.000393 & 0.0004 & 0.000398 & 0.0004 & 0.0000 & 0.000403 & 0.000417 & 0.000245 & 0.0004 & 0.0001 & & & & & \\
\hline ט & & A.077 & & & & & & ND & ND & ND & & & & & & & \\
\hline$\tilde{\sigma}$ & & A 078 & & & & & & & & & & & & & & & \\
\hline $\bar{D}$ & & A081 & 0 & 0 & 0 & 0.0000 & 0.0000 & 0.052 & 0.053 & 0.053 & 0.0527 & 0.0006 & & & & & \\
\hline 曰 & & A 082 & & & & & & & & & & & 0.05 & & & 0.050 & \\
\hline 10 & & A083 & $<0.33$ & & & $<033$ & & $<0.33$ & & & $<033$ & & & & & & \\
\hline D & & A 084 & $<0.0125$ & $<0.0125$ & $<0.0125$ & & & $<0.0125$ & $<0.0125$ & $<0.0125$ & & & & & & & \\
\hline$\stackrel{0}{n}$ & & A085 & $<0.025$ & $<0.025$ & $<0.025$ & & & $<0.025$ & $<0.025$ & $<0.025$ & & & & & & & \\
\hline 0 & & A 086 & BLQ & BLQ & BLQ & & & BLQ & BLQ & BLQ & & & & & & & \\
\hline 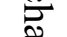 & 昰 & A087 & & & & & & & & & & & & & & & \\
\hline 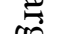 & 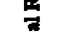 & A 088 & & & & & & & & & & & & & & & \\
\hline 0 & 忌 & A089 & 1.46 & 15 & 1.47 & 14767 & 0.0208 & 266 & 258 & 2.07 & 24367 & 03201 & & & & & \\
\hline$\rightarrow$ & $\frac{3}{8}$ & A 090 & & & & & & & & & & & 0.0002 & 0.0002 & 0.0002 & 0.0002 & 0.000 \\
\hline $\overrightarrow{0}$ & & A091 & & & & & & & & & & & & & & & \\
\hline 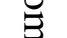 & & A 092 & & & & & & & & & & & & & & & \\
\hline F & & A093 & & & & & & & & & & & & & & & \\
\hline$\underline{\Xi}$ & & A 094 & & & & & & & & & & & & & & & \\
\hline 릉 & & A095 & $<0.0177$ & $<0.0177$ & $<0.0177$ & $<0.0177$ & & $<0.0177$ & $<0.0177$ & $<0.0177$ & $<0.0177$ & & & & & & \\
\hline 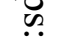 & & A 096 & & & & & & & & & & & & & & & \\
\hline 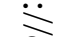 & & A097 & $<0.0100$ & $<0.0100$ & $<0.0100$ & $<0.0100$ & & $<0.0100$ & $<0.0100$ & $<0.0100$ & $<0.0100$ & & & & & & \\
\hline 를 & & A 098 & 0.005 & & & 0.0050 & & $<0.0025$ & & & $<0.0025$ & & & & & & \\
\hline$\stackrel{0}{.}$ & & $\mathbf{A} 099$ & $0 \_021$ & 0.021 & 0.019 & 0.0203 & 0.0012 & 0.023 & 0.023 & 0.019 & 0.0217 & 0.0023 & & & & & \\
\hline c & & A 100 & $<0.0210$ & $<0.0210$ & $<0.0210$ & $<0.0210$ & & $<0.0210$ & $<0.0210$ & $<0.0210$ & $<0.0210$ & & & & & & \\
\hline $\overrightarrow{0}$ & & A 101 & & & & & & & & & & & & & & & \\
\hline$\Xi$ & & A 102 & $<0.04$ & $<0.04$ & $<0.04$ & $<0.04$ & & $<0.04$ & $<0.04$ & $<0.04$ & $<0.04$ & & & & & & \\
\hline 0 & & A 103 & 0 & 0 & 0 & 0.0000 & 0.0000 & 0.091 & 0.128 & 0.128 & 0.1157 & 0.0214 & & & & & \\
\hline a & & A 104 & $<0.033$ & $<0.026$ & $<0.034$ & $<0.033$ & & $<0.033$ & $<0.026$ & $<0.034$ & $<0.033$ & & & & & & \\
\hline 0 & & A 105 & $<0.025$ & $<0.025$ & $<0.025$ & $<0.025$ & & 0.06 & 0.06 & 0.06 & 0.0600 & 0.0000 & & & & & \\
\hline$\infty$ & & A 106 & $<0.05$ & $<0.05$ & $<0.05$ & $<0.05$ & & $<0.05$ & $<0.05$ & $<0.05$ & $<0.05$ & & & & & & \\
\hline$\infty$ & & A 107 & 0.0123 & 0.012 & 0.0123 & 0.0122 & 0.0002 & 0.012 & 0.0121 & 0.0116 & 0.0119 & 0.0003 & & & & & \\
\hline$Z$ & & A 108 & & & & & & & & & & & & & & & \\
\hline $\bar{U}$ & & A 109 & & & & & & & & & & & & & & & \\
\hline 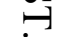 & & A 110 & $<0.01$ & $<0.01$ & $<0.01$ & $<0.01$ & & $<0.01$ & $<0.01$ & $<0.01$ & $<0.01$ & & & & & & \\
\hline$\exists$ & & A111 & 0.017 & 0.017 & 0.018 & 0.0173 & 0.0006 & 0.005 & 0.005 & 0.005 & 0.0050 & 0.0000 & & & & & \\
\hline 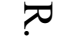 & & A 112 & & & & & & & & & & & & & & & \\
\hline$\infty$ & & A113 & 0.024324 & 0.024052 & 0.023356 & 0.0239 & 0.0005 & 0 & 0 & 0 & 0.0000 & 0.0000 & & & & & \\
\hline$\infty$ & & A 114 & & & & & & 0.0812 & 0.084 & 0.086 & 0.0837 & 0.0024 & & & & & \\
\hline u & & A115 & & & & & & & & & & & $<0.01$ & $<0.01$ & $<0.01$ & $<0.01$ & \\
\hline & & A.116 & 0 & 0.0156 & 0.0179 & 0.0112 & 0.0097 & 0 & 0 & 0.0086 & 0.0029 & 0.0050 & & & & & \\
\hline & 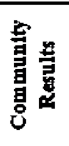 & & $\begin{array}{l}\text { Consensus } \\
\text { Consensus } \\
\text { Maximum } \\
\text { Minimum } \\
\mathrm{N}\end{array}$ & $\begin{array}{l}\text { is Mean } \\
\text { s Standard I }\end{array}$ & Deviation & $\begin{array}{c}0.0056 \\
0.0016 \\
14767 \\
0.0000 \\
23\end{array}$ & & $\begin{array}{l}\text { Consensus } \\
\text { Consensus } \\
\text { Maximum } \\
\text { Minimum } \\
\text { N }\end{array}$ & $\begin{array}{l}\text { s Mean } \\
\text { s Standard I }\end{array}$ & Deviation & $\begin{array}{c}0.0115 \\
0.0032 \\
24367 \\
0.0000 \\
31\end{array}$ & & $\begin{array}{l}\text { Consensu } \\
\text { Consensu } \\
\text { Maximum } \\
\text { Minimum } \\
\text { N }\end{array}$ & $\begin{array}{l}\text { s Mean } \\
\text { s Standand }\end{array}$ & Deviation & $\begin{array}{c}0.021 \\
0.018 \\
0.050 \\
0.000 \\
2\end{array}$ & \\
\hline
\end{tabular}




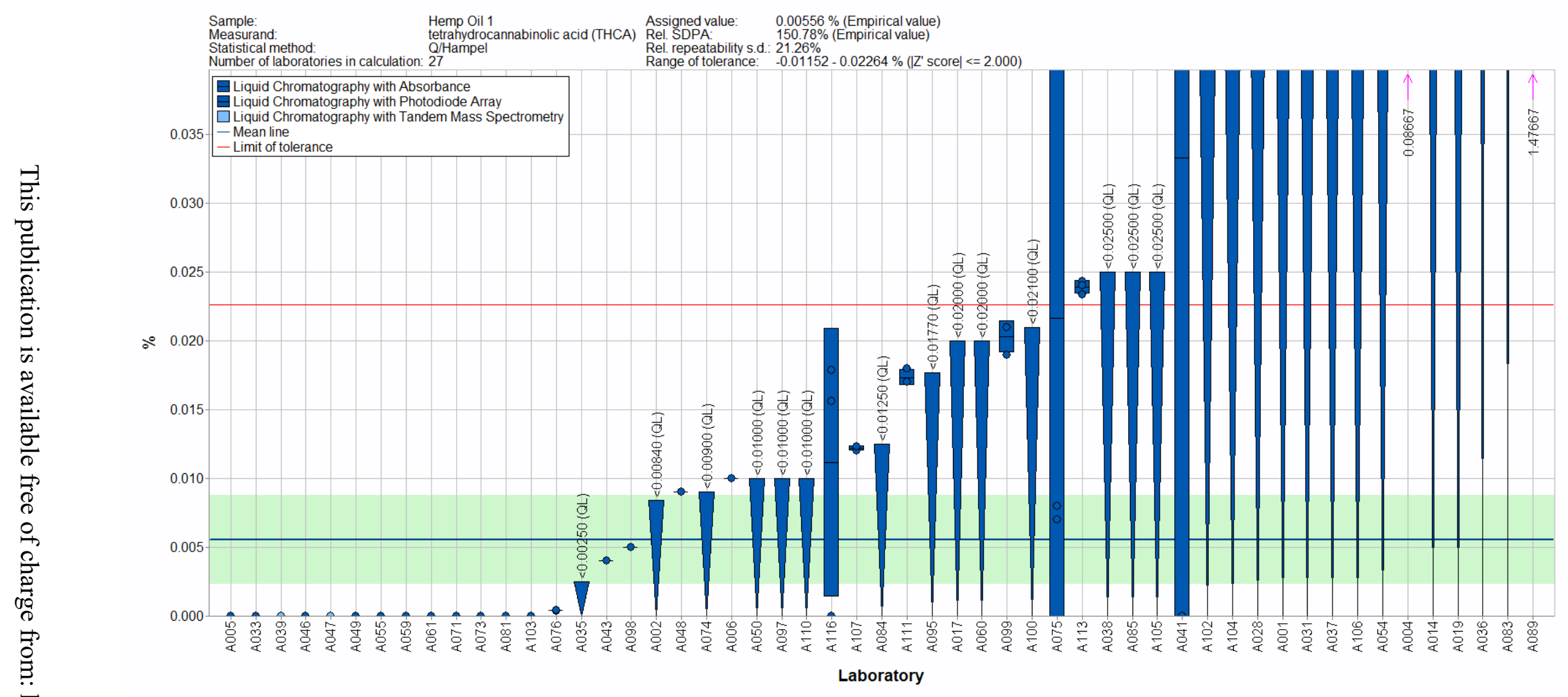

Figure 2-5. THCA in Hemp Oil 1 (data summary view - analytical method). In this view, individual laboratory data are plotted (circles) with the individual laboratory standard deviation (rectangle). The color of the data point represents the analytical method employed. The solid blue line represents the consensus mean, and the green shaded region represents the $95 \%$ confidence interval for the consensus mean. The solid red line represents the upper consensus range of tolerance, calculated as the values above the consensus mean that results in an acceptable $Z_{\text {comm }}^{\prime}$ score, $\left|Z_{\text {comm }}^{\prime}\right| \leq 2$, with the lower limit set at zero. A NIST value has not been determined in this material. The downward triangle represents data reported as a threshold or LOQ value. 


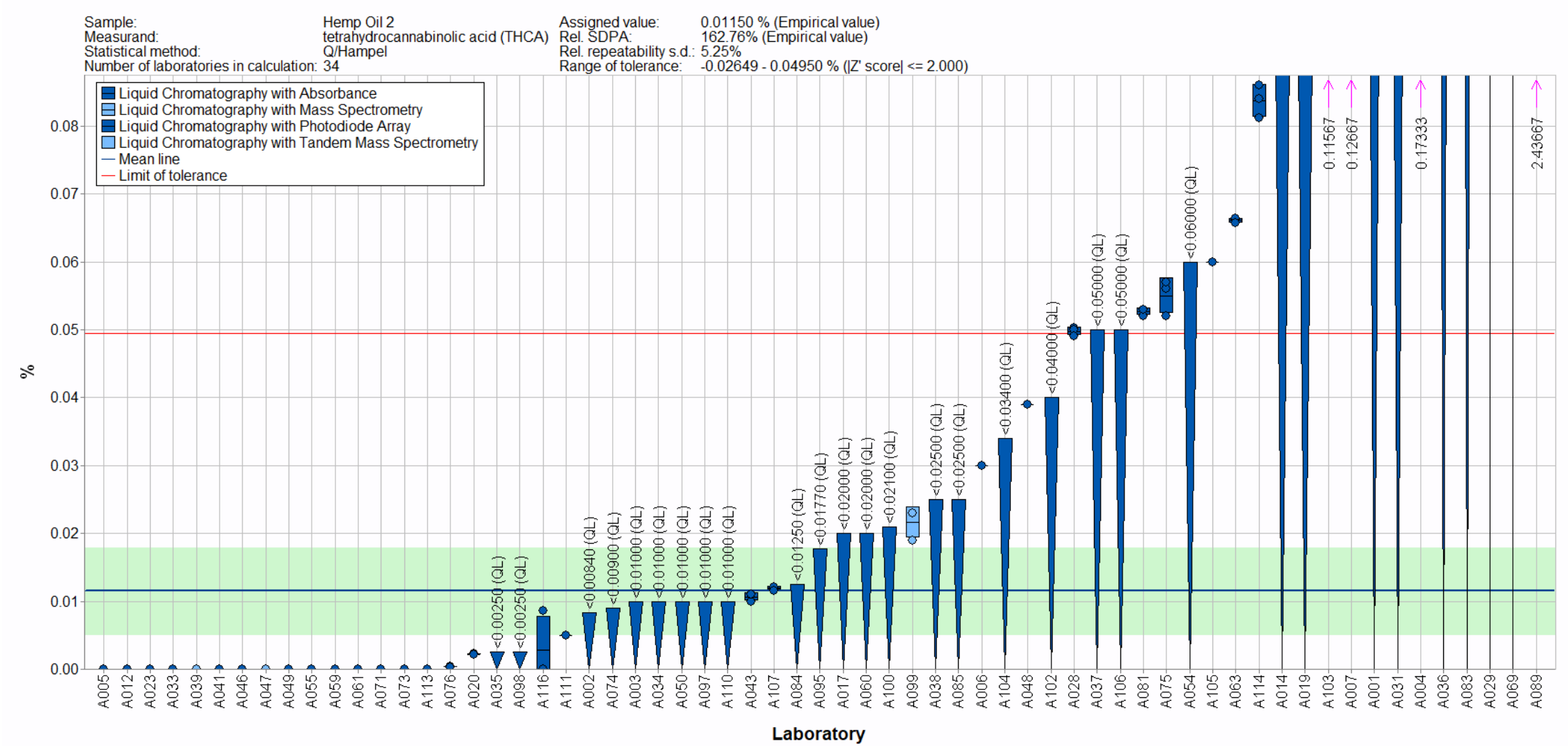

Figure 2-6. THCA in Hemp Oil 2 (data summary view - analytical method). In this view, individual laboratory data are plotted (circles) with the individual laboratory standard deviation (rectangle). The color of the data point represents the analytical method employed. The solid blue line represents the consensus mean, and the green shaded region represents the $95 \%$ confidence interval for the consensus mean. The solid red line represents the upper consensus range of tolerance, calculated as the values above the consensus mean that results in an acceptable $Z_{\text {comm }}^{\prime}$ score, $\left|Z_{\text {comm }}^{\prime}\right| \leq 2$, with the lower limit set at zero. A NIST value has not been determined in this material. The downward triangle represents data reported as a threshold or LOQ value. 


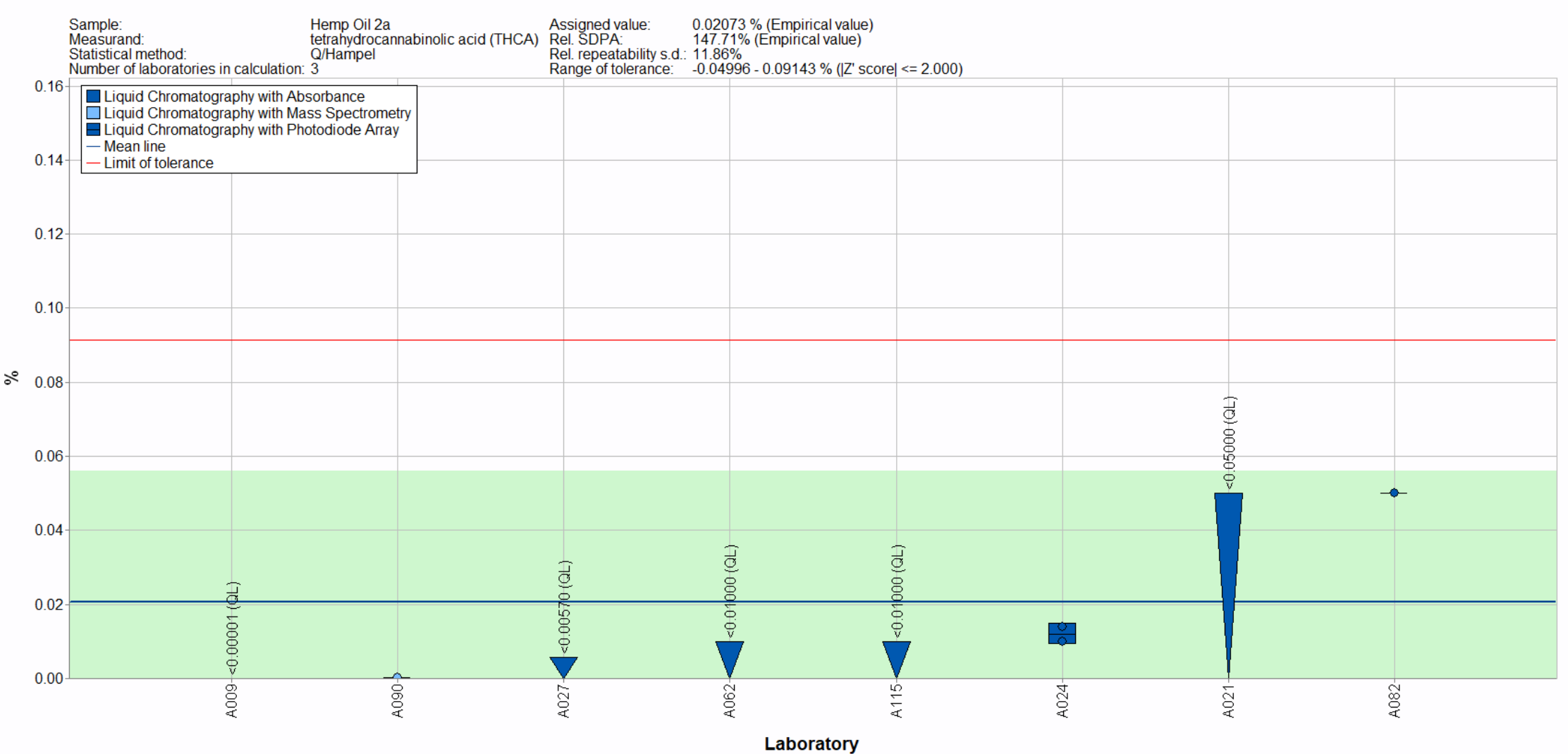

Figure 2-7. THCA in Hemp Oil 2a (data summary view - analytical method). In this view, individual laboratory data are plotted (circles) with the individual laboratory standard deviation (rectangle). The color of the data point represents the analytical method employed. The solid blue line represents the consensus mean, and the green shaded region represents the $95 \%$ confidence interval for the consensus mean. The solid red line represents the upper consensus range of tolerance, calculated as the values above the consensus mean that results in an acceptable $Z_{\text {comm }}^{\prime}$ score, $\left|Z_{\text {comm }}^{\prime}\right| \leq 2$, with the lower limit set at zero. A NIST value has not been determined in this material. The downward triangle represents data reported as a threshold or LOQ value. 


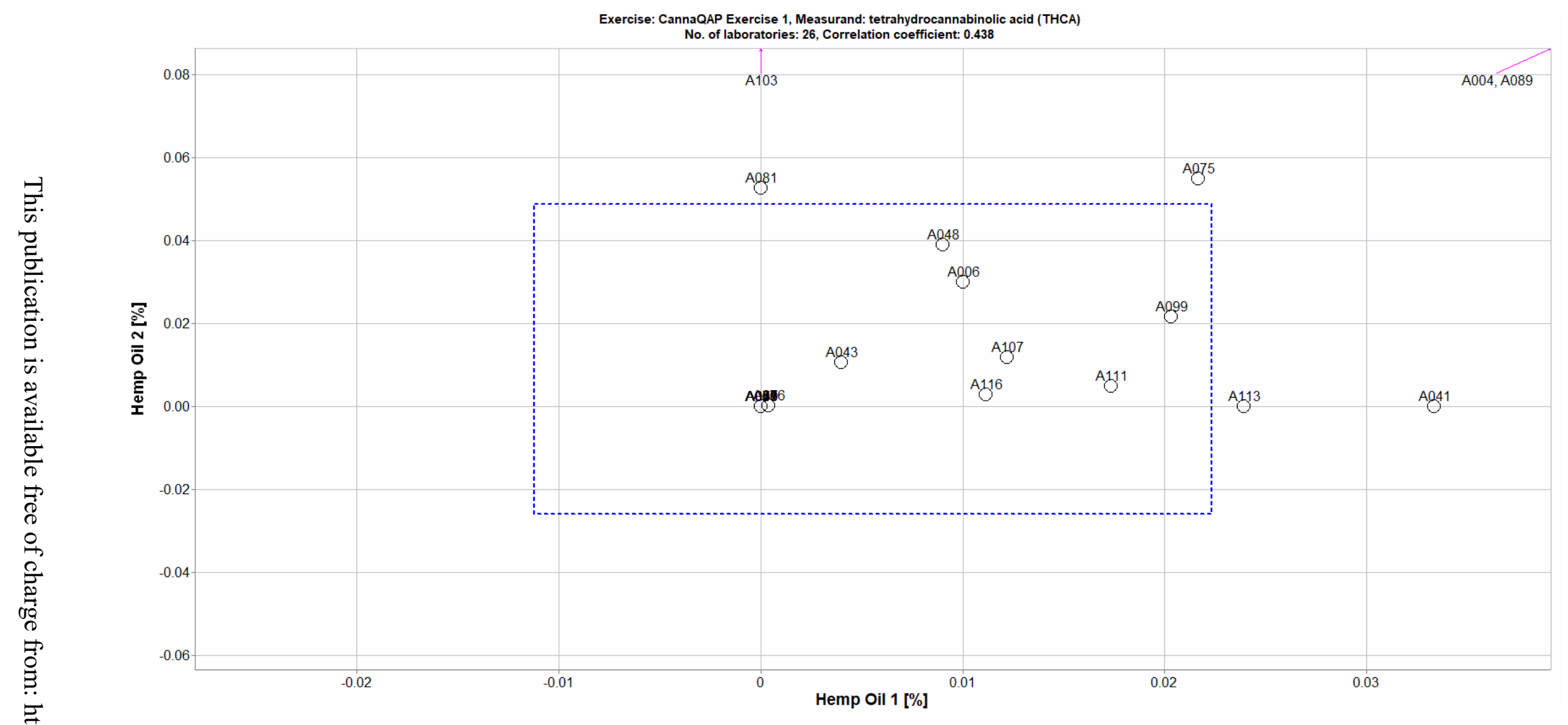

Figure 2-8. Laboratory means for THCA in Hemp Oil 1 and Hemp Oil 2 (sample/sample comparison view). In this view, the individual laboratory mean for one sample (Hemp Oil 1) is compared to the individual laboratory mean for a second sample (Hemp Oil 2). The dotted blue box represents the consensus range of tolerance for Hemp Oil 1 (x-axis) and Hemp Oil 2 (y-axis), calculated as the values above and below the consensus means that result in an acceptable $Z_{\text {comm }}^{\prime}$ score, $\left|Z_{\text {comm }}^{\prime}\right| \leq 2$. 
Table 2-4. Data summary table for $\Delta^{8}$-THC in hemp oils. Data highlighted in red have been flagged as a data entry of zero or results that include text (e.g., "< LOQ" or "present"). Data highlighted in blue have been identified as outside the consensus tolerance limits and would be estimated to result in an unacceptable $Z_{\text {comm }}^{\prime}$ score, $\left|Z_{\text {comm }}^{\prime}\right| \geq 2$. Note: This table spans two pages; the NIST values and consensus values are included on both pages for convenience.

\begin{tabular}{|c|c|c|c|c|c|c|c|c|c|c|c|c|c|c|c|c|}
\hline & & \multicolumn{15}{|c|}{$\Delta 8$-Tetrahydrocannabinol ( $\triangle 8$-THC) } \\
\hline & & \multicolumn{5}{|c|}{ Hemp Oil 1 (mass \%) } & \multicolumn{5}{|c|}{ Hemp Oil 2 (mass \%) } & \multicolumn{5}{|c|}{ Hemp Oil 2a (mass \%) } \\
\hline & Lab & $\mathbf{A}$ & $\mathbf{B}$ & $\mathbf{C}$ & Avg & SD & $\mathbf{A}$ & B & $\mathbf{C}$ & Avg & SD & $\mathbf{A}$ & $\mathbf{B}$ & $\mathbf{C}$ & Avg & SD \\
\hline \multirow{42}{*}{ 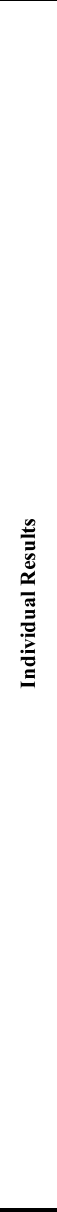 } & NIST & & & & & & & & & & & & & & & \\
\hline & A002 & $<0.0104$ & $<0.0104$ & $<0.0104$ & $<0.0104$ & & $<0.0104$ & $<0.0104$ & $<0.0104$ & $<0.0104$ & & & & & & \\
\hline & A003 & & & & & & $<0.01$ & $<0.01$ & $<0.01$ & $<0.01$ & & & & & & \\
\hline & A005 & 0 & 0 & 0 & 0.0000 & 0.0000 & 0 & 0 & 0 & 0.0000 & 0.0000 & & & & & \\
\hline & A007 & & & & & & & & & & & & & & & \\
\hline & A008 & 0.044 & & & 0.0440 & & & & & & & & & & & \\
\hline & A009 & & & & & & & & & & & $<0.00268$ & $<0.002687$ & $<0.00268$ & $<0.00268$ & \\
\hline & A010 & & & & & & & & & & & & & & & \\
\hline & A011 & & & & & & & & & & & & & & & \\
\hline & A012 & & & & & & 0 & 0 & 0 & 0.0000 & 0.0000 & & & & & \\
\hline & A013 & & & & & & 0.074 & 0.048 & 0.078 & 0.0667 & 0.0163 & & & & & \\
\hline & A014 & $<0.09$ & & & $<0.09$ & & $<0.09$ & & & $<0.09$ & & & & & & \\
\hline & A015 & & & & & & & & & & & & & & & \\
\hline & A016 & & & & & & & & & & & & & & & \\
\hline & A017 & $<0.02$ & & & $<0.02$ & & 0.02 & & & 0.0200 & & & & & & \\
\hline & A019 & $<0.09$ & & & $<0.09$ & & $<0.09$ & & & $<0.09$ & & & & & & \\
\hline & $\mathrm{A} 020$ & & & & & & & & & & & & & & & \\
\hline & A021 & & & & & & & & & & & $<0.05$ & $<0.05$ & $<0.05$ & $<0.05$ & \\
\hline & A022 & & & & & & & & & & & & & & & \\
\hline & A023 & & & & & & 0 & 0 & 0 & 0.0000 & 0.0000 & & & & & \\
\hline & A024 & & & & & & & & & & & 0.011 & 0.023 & 0.012 & 0.02 & 0.01 \\
\hline & A025 & & & & & & & & & & & & & & & \\
\hline & A026 & & & & & & & & & & & & & & & \\
\hline & A027 & & & & & & & & & & & $<0.0057$ & $<0.0057$ & $<0.0057$ & $<0.0057$ & \\
\hline & A 030 & $<0.01$ & & & $<0.01$ & & $<0.01$ & & & $<0.01$ & & & & & & \\
\hline & A031 & & & & & & & & & & & & & & & \\
\hline & A 033 & 0 & 0 & 0 & 0.0000 & 0.0000 & 0 & 0 & 0 & 0.0000 & 0.0000 & & & & & \\
\hline & A034 & & & & & & & & & & & & & & & \\
\hline & A035 & 0.0147 & & & 0.0147 & & 0.0257 & 0.0286 & 0.0246 & 0.0263 & 0.0021 & & & & & \\
\hline & A036 & $<0.206$ & $<0.206$ & $<0.206$ & & & $<0.247$ & $<0.247$ & $<0.247$ & $<0.247$ & & & & & & \\
\hline & A038 & $<0.025$ & $<0.025$ & $<0.025$ & & & $<0.025$ & $<0.025$ & $<0.025$ & $<0.025$ & & & & & & \\
\hline & A039 & 0 & 0 & 0 & 0.0000 & 0.0000 & 0 & 0 & 0 & 0.0000 & 0.0000 & & & & & \\
\hline & A 040 & & & & & & & & & & & ND & ND & ND & & \\
\hline & A041 & 0 & 0 & 0 & 0.0000 & 0.0000 & 0 & 0 & 0 & 0.0000 & 0.0000 & & & & & \\
\hline & A 043 & 0.01 & 0.009 & 0.007 & 0.0087 & 0.0015 & 0.015 & 0.015 & 0.015 & 0.0150 & 0.0000 & & & & & \\
\hline & A044 & & & & & & & & & & & & & & & \\
\hline & A045 & & & & & & & & & & & & & & & \\
\hline & A046 & 0 & 0 & 0 & 0.0000 & 0.0000 & 0 & 0 & 0 & 0.0000 & 0.0000 & & & & & \\
\hline & A050 & $<0.01$ & $<0.01$ & $<0.01$ & $<0.01$ & & $<0.01$ & $<0.01$ & $<0.01$ & $<0.01$ & & & & & & \\
\hline & A052 & & & & & & & & & & & & & & & \\
\hline & A053 & & & & & & & & & & & & & & & \\
\hline & $\mathrm{A} 055$ & 0 & 0 & 0 & 0.0000 & 0.0000 & 0 & 0 & 0 & 0.0000 & 0.0000 & & & & & \\
\hline \multirow{4}{*}{ 急事 } & & \multirow{4}{*}{\multicolumn{3}{|c|}{$\begin{array}{l}\text { Consensus Mean } \\
\text { Consensus Standard Deviation } \\
\text { Maximum } \\
\text { Minimum } \\
\mathrm{N} \\
\end{array}$}} & 0.0104 & & \multirow{4}{*}{\multicolumn{2}{|c|}{$\begin{array}{l}\text { Consensus Mean } \\
\text { Consensus Standard } \\
\text { Maximum } \\
\text { Minimum } \\
\mathrm{N} \\
\end{array}$}} & & 0.0139 & & \multirow{4}{*}{\begin{tabular}{|l} 
Consensus \\
Consensus \\
Maximum \\
Minimum \\
$\mathrm{N}$ \\
\end{tabular}} & s Mean & & 0.04 & \\
\hline & & & & & 0.0030 & & & & Deviation & 0.0041 & & & s Standard & Deviation & 0.02 & \\
\hline & & & & & 0.0467 & & & & & 0.1067 & & & & & 0.13 & \\
\hline & & & & & $\begin{array}{c}0.0000 \\
15\end{array}$ & & & & & $\begin{array}{c}0.0000 \\
22\end{array}$ & & & & & $\begin{array}{c}0.00 \\
2\end{array}$ & \\
\hline
\end{tabular}




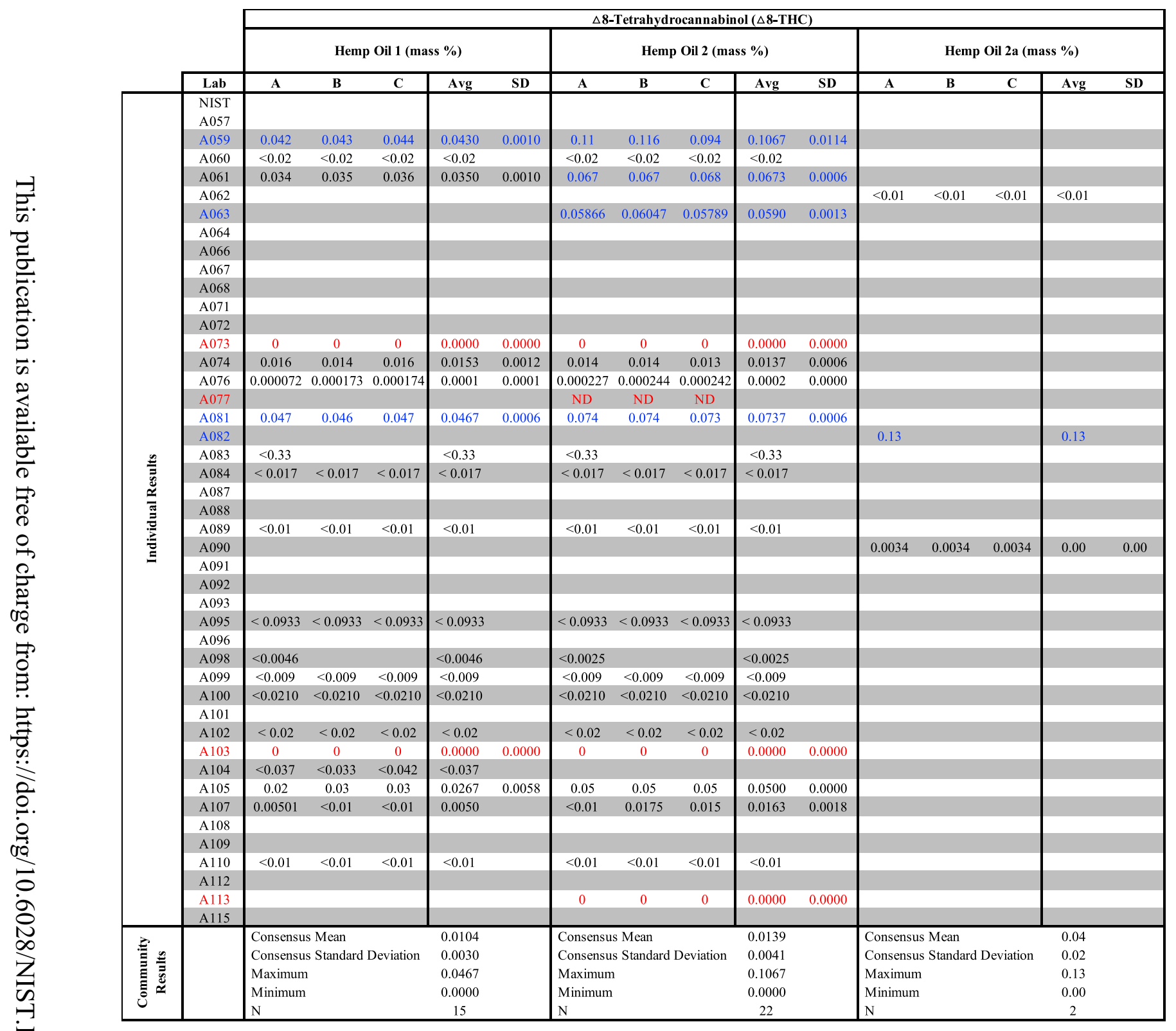




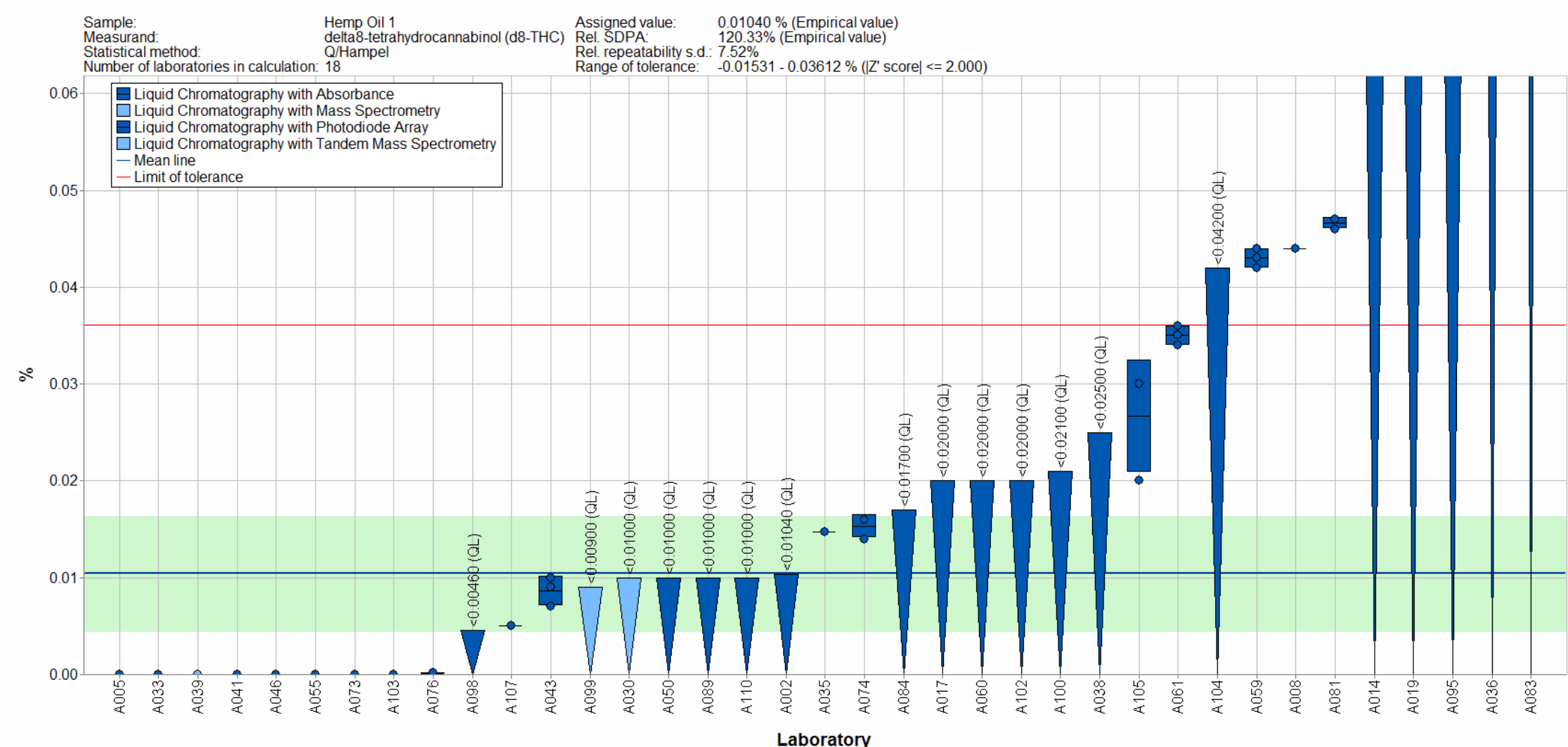

Figure 2-9. $\Delta^{8}$-THC in Hemp Oil 1 (data summary view - analytical method). In this view, individual laboratory data are plotted (circles) with the individual laboratory standard deviation (rectangle). The color of the data point represents the analytical method employed. The solid blue line represents the consensus mean, and the green shaded region represents the $95 \%$ confidence interval for the consensus mean. The solid red line represents the upper consensus range of tolerance, calculated as the values above the consensus mean that results in an acceptable $Z_{\text {comm }}^{\prime}$ score, $\left|Z_{\text {comm }}^{\prime}\right| \leq 2$, with the lower limit set at zero. A NIST value has not been determined in this material. The downward triangle represents data reported as a threshold or LOQ value. 


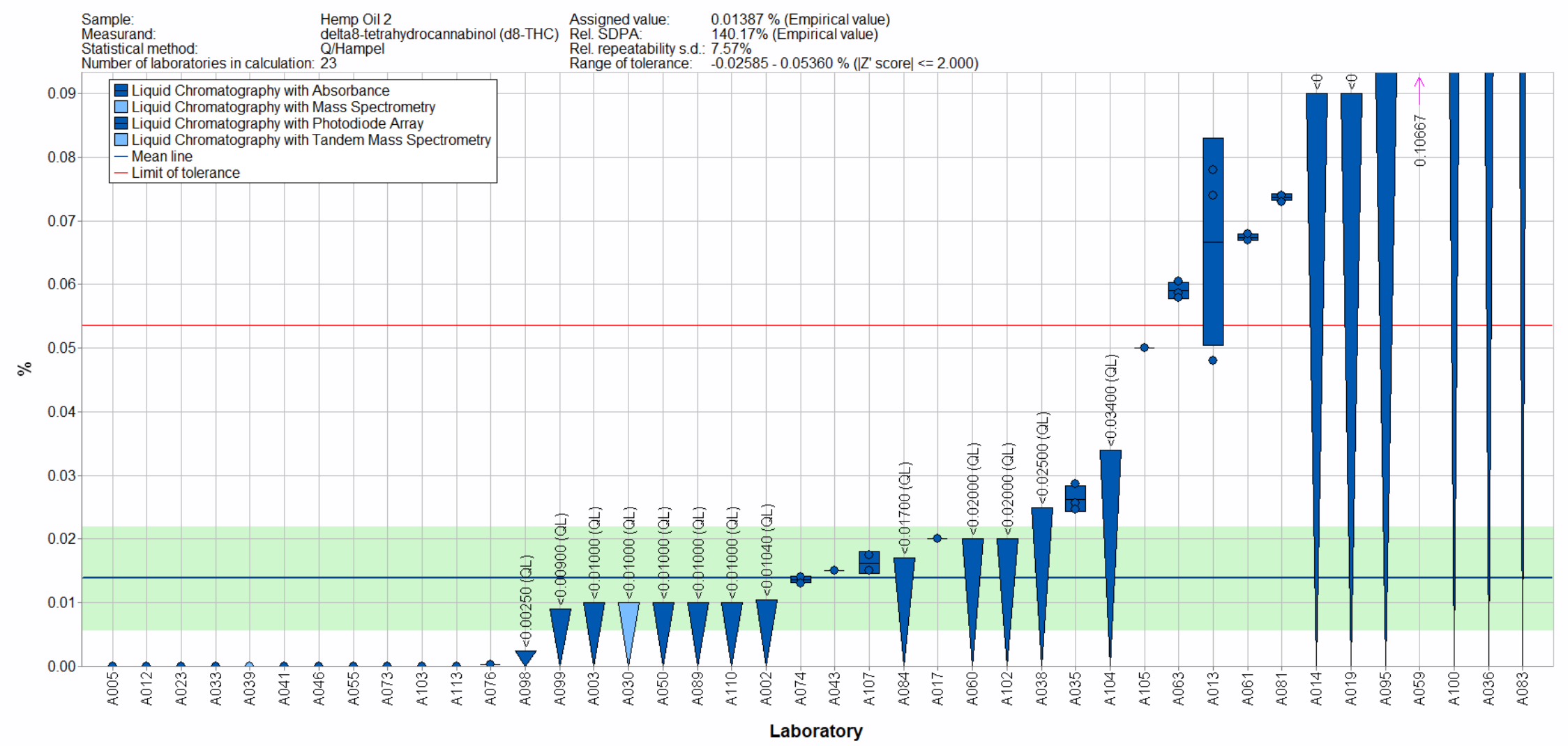

Figure 2-10. $\Delta^{8}$-THC in Hemp Oil 2 (data summary view - analytical method). In this view, individual laboratory data are plotted (circles) with the individual laboratory standard deviation (rectangle). The color of the data point represents the analytical method employed. The solid blue line represents the consensus mean, and the green shaded region represents the $95 \%$ confidence interval for the consensus mean. The solid red line represents the upper consensus range of tolerance, calculated as the values above the consensus mean that results in an acceptable $Z_{\text {comm }}^{\prime}$ score, $\left|Z_{\text {comm }}^{\prime}\right| \leq 2$, with the lower limit set at zero. A NIST value has not been determined in this material. The downward triangle represents data reported as a threshold or LOQ value. 


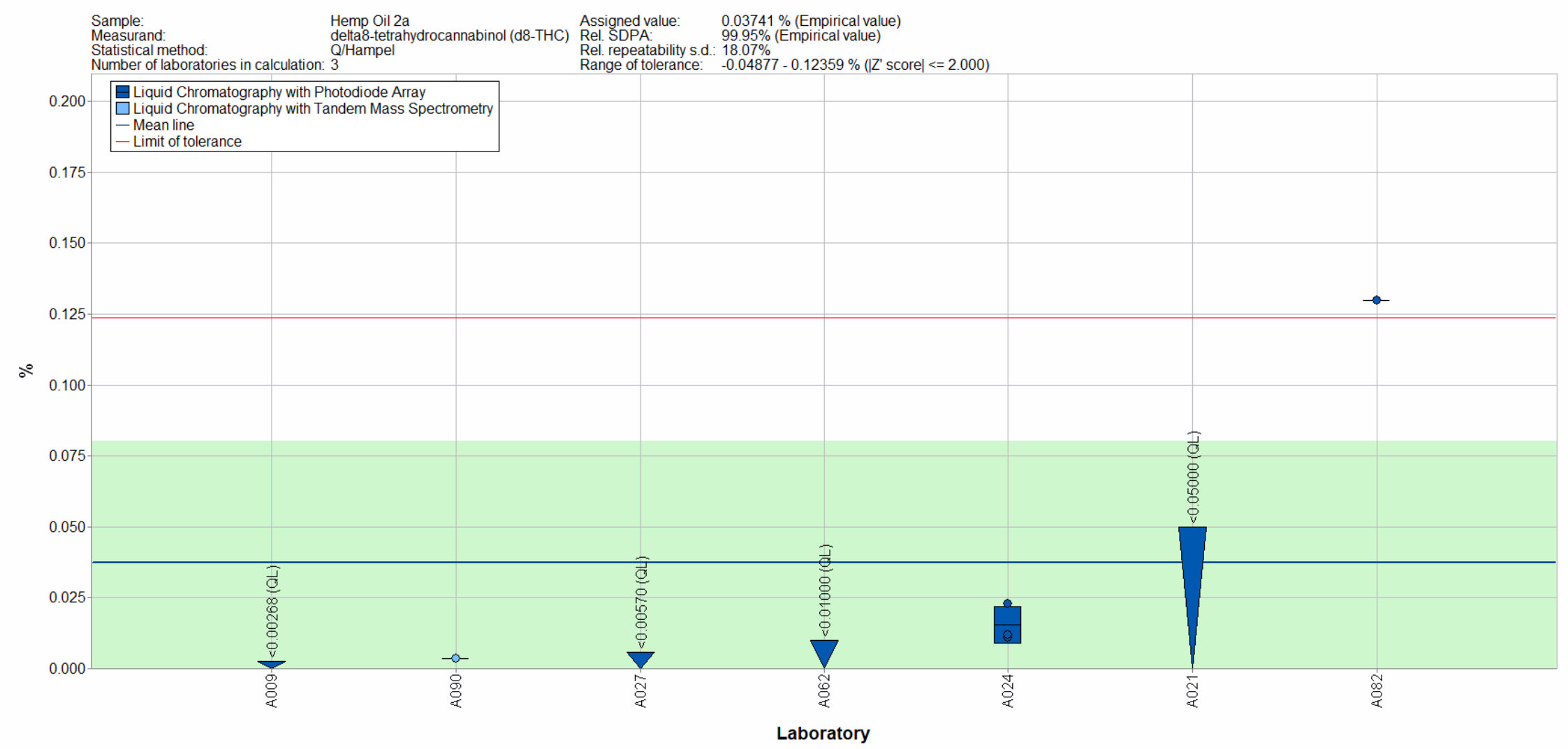

Figure 2-11. $\Delta^{8}$-THC in Hemp Oil 2a (data summary view - analytical method). In this view, individual laboratory data are plotted (circles) with the individual laboratory standard deviation (rectangle). The color of the data point represents the analytical method employed. The solid blue line represents the consensus mean, and the green shaded region represents the $95 \%$ confidence interval for the consensus mean. The solid red line represents the upper consensus range of tolerance, calculated as the values above the consensus mean that results in an acceptable $Z_{\text {comm }}^{\prime}$ score, $\left|Z_{\text {comm }}^{\prime}\right| \leq 2$, with the lower limit set at zero. A NIST value has not been determined in this material. The downward triangle represents data reported as a threshold or LOQ value. 


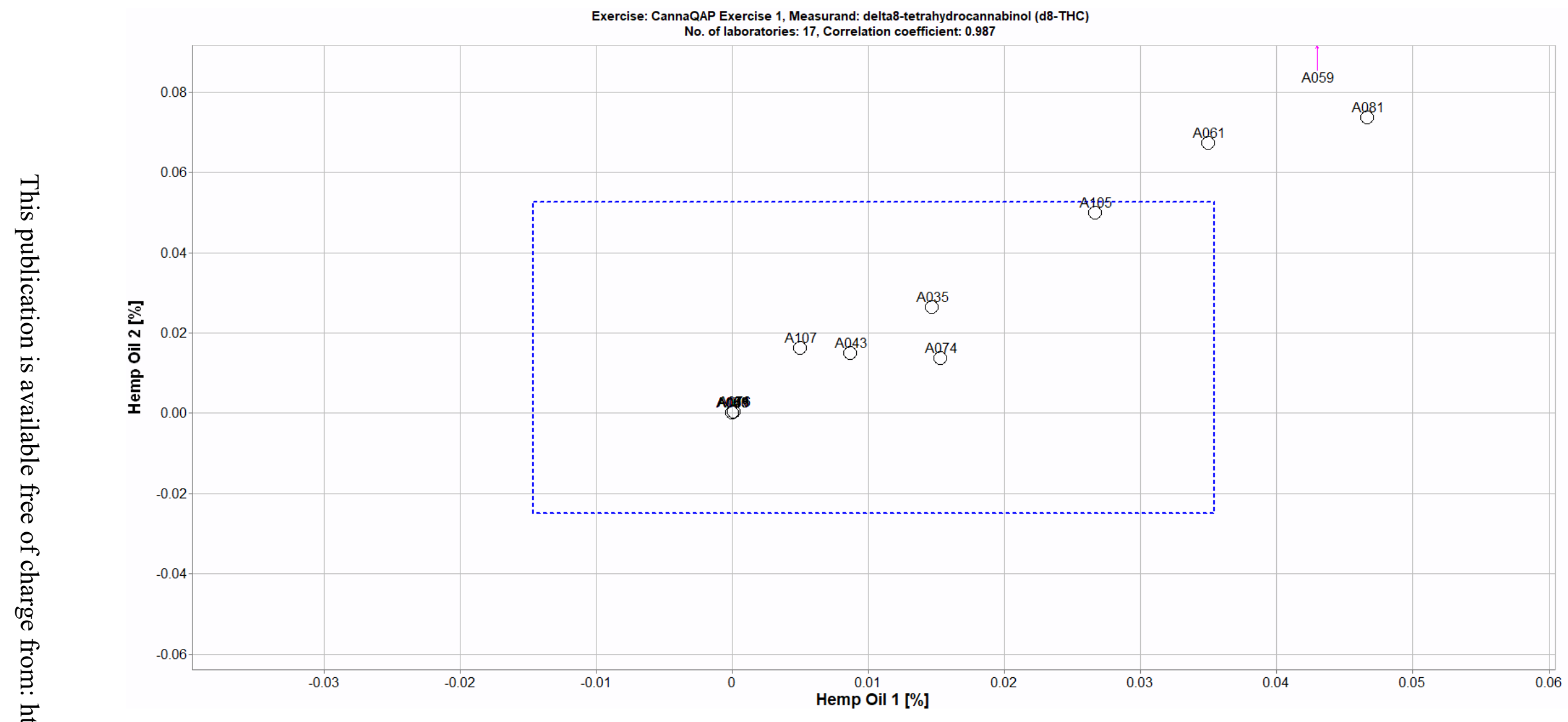

Figure 2-12. Laboratory means for $\Delta^{8}$-THC in Hemp Oil 1 and Hemp Oil 2 (sample/sample comparison view). In this view, the individual laboratory mean for one sample (Hemp Oil 1) is compared to the individual laboratory mean for a second sample (Hemp Oil 2). The dotted blue box represents the consensus range of tolerance for Hemp Oil 1 (x-axis) and Hemp Oil 2 (y-axis), calculated as the values above and below the consensus means that result in an acceptable $Z_{\text {comm }}^{\prime}$ score, $\left|Z_{\text {comm }}^{\prime}\right| \leq 2$. 
Table 2-5. Data summary table for total $\mathrm{THC}$ in hemp oils. Data highlighted in red have been flagged as a data entry of zero or results that include text (e.g., "< LOQ" or "present"). Data highlighted in blue have been identified as outside the consensus tolerance limits and would be estimated to result in an unacceptable $Z_{\text {comm }}^{\prime}$ score, $\left|Z_{\text {comm }}^{\prime}\right| \geq 2$. Note: This table spans two pages; the NIST values and consensus values are included on both pages for convenience.

\begin{tabular}{|c|c|c|c|c|c|c|c|c|c|c|c|c|c|c|c|c|}
\hline & \multirow[b]{3}{*}{ Lab } & \multicolumn{15}{|c|}{ Total $\triangle 9$-THC } \\
\hline & & \multicolumn{5}{|c|}{ Hemp Oil 1 (mass \%) } & \multicolumn{5}{|c|}{ Hemp Oil 2 (mass \%) } & \multicolumn{5}{|c|}{ Hemp Oil 2a (mass \%) } \\
\hline & & $\mathbf{A}$ & B & $\mathbf{C}$ & Avg & SD & $\mathbf{A}$ & B & $\mathbf{C}$ & Avg & SD & $\mathbf{A}$ & $\mathbf{B}$ & $\mathrm{C}$ & Avg & SD \\
\hline \multirow{46}{*}{ 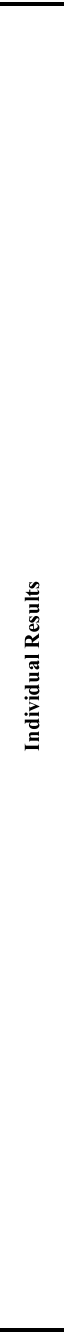 } & NIST & & & & 0.131 & 0.010 & & & & 0.160 & 0.018 & & & & 0.15 & 0.024 \\
\hline & A001 & 0.14 & 0.13 & 0.12 & 0.1300 & 0.0100 & 0.17 & 0.18 & 0.17 & 0.1733 & 0.0058 & & & & & \\
\hline & A002 & 0.13898 & 0.1326 & 0.12578 & 0.1325 & 0.0066 & 0.170171 & 0.171068 & 0.156471 & 0.1659 & 0.0082 & & & & & \\
\hline & A003 & & & & & & 0.174 & 0.163 & 0.157 & 0.1647 & 0.0086 & & & & & \\
\hline & A004 & 0.21 & 0.18 & 0.17 & 0.1867 & 0.0208 & 0.32 & 0.3 & 0.25 & 0.2900 & 0.0361 & & & & & \\
\hline & A005 & 0.14 & 0.125 & 0.135 & 0.1333 & 0.0076 & 0.17 & 0.169 & 0.172 & 0.1703 & 0.0015 & & & & & \\
\hline & A007 & & & & & & 0.08 & 0.11 & 0.08 & 0.0900 & 0.0173 & & & & & \\
\hline & A008 & & & & & & & & & & & & & & & \\
\hline & A009 & & & & & & & & & & & 0.164 & 0.161 & 0.176 & 0.167 & 0.008 \\
\hline & A010 & & & & & & & & & & & & & & & \\
\hline & A011 & & & & & & & & & & & & & & & \\
\hline & A013 & 0.134 & 0.141 & 0.144 & 0.1397 & 0.0051 & 0.151 & 0.147 & 0.145 & 0.1477 & 0.0031 & & & & & \\
\hline & A014 & & & & & & & & & & & & & & & \\
\hline & A015 & & & & & & & & & & & 0.18 & 0.12 & 0.28 & 0.193 & 0.081 \\
\hline & A016 & & & & & & & & & & & & & & & \\
\hline & A017 & & & & & & & & & & & & & & & \\
\hline & A018 & & & & & & & & & & & & & & & \\
\hline & A019 & & & & & & & & & & & & & & & \\
\hline & A020 & 0.12328 & 0.124478 & 0.123915 & 0.1239 & 0.0006 & 0.146315 & 0.14812 & 0.162433 & 0.1523 & 0.0088 & & & & & \\
\hline & A021 & & & & & & & & & & & 0.05 & 0.08 & 0.07 & 0.067 & 0.015 \\
\hline & A022 & & & & & & & & & & & 0.1199 & 0.1218 & 0.1226 & 0.121 & 0.001 \\
\hline & A 023 & & & & & & 0.1835 & 0.1795 & 0.1822 & 0.1817 & 0.0020 & & & & & \\
\hline & A 024 & & & & & & & & & & & 0.17 & 0.193 & 0.184 & 0.182 & 0.012 \\
\hline & A026 & $<0.15$ & $<0.15$ & $<0.15$ & $<0.15$ & & $<0.15$ & $<0.15$ & $<0.15$ & $<0.15$ & & & & & & \\
\hline & A027 & & & & & & & & & & & 0.156 & 0.158 & 0.158 & 0.157 & 0.001 \\
\hline & A028 & 0.1538 & 0.1718 & 0.1537 & 0.1598 & 0.0104 & 0.2638 & 0.231 & 0.2584 & 0.2511 & 0.0176 & & & & & \\
\hline & $\mathrm{A} 030$ & 0.186 & & & 0.1860 & & 0.182 & & & 0.1820 & & & & & & \\
\hline & A 031 & 0.12 & 0.12 & 0.12 & 0.1200 & 0.0000 & $<0.15$ & $<0.15$ & $<0.15$ & $<0.15$ & & & & & & \\
\hline & A033 & 0.12 & 0.11 & 0.12 & 0.1167 & 0.0058 & 0.14 & 0.15 & 0.13 & 0.1400 & 0.0100 & & & & & \\
\hline & A034 & & & & & & 0.136 & 0.135 & 0.135 & 0.1353 & 0.0006 & & & & & \\
\hline & A035 & 0.129 & & & 0.1290 & & 0.2 & 0.203 & 0.202 & 0.2017 & 0.0015 & & & & & \\
\hline & A036 & 0.135 & 0.137 & 0.14 & 0.1373 & 0.0025 & 0.181 & 0.182 & 0.187 & 0.1833 & 0.0032 & & & & & \\
\hline & A 037 & & & & & & & & & & & & & & & \\
\hline & A038 & 0.161 & 0.16 & 0.158 & 0.1597 & 0.0015 & 0.207 & 0.192 & 0.208 & 0.2023 & 0.0090 & & & & & \\
\hline & A039 & 0.09 & 0.07 & 0.08 & 0.0800 & 0.0100 & 0.1 & 0.1 & 0.09 & 0.0967 & 0.0058 & & & & & \\
\hline & A 040 & & & & & & & & & & & 0.12641 & 0.12562 & 0.12734 & 0.126 & 0.001 \\
\hline & A043 & 0.204 & 0.194 & 0.203 & 0.2003 & 0.0055 & 0.315 & 0.319 & 0.32 & 0.3180 & 0.0026 & & & & & \\
\hline & A044 & & & & & & & & & & & & & & & \\
\hline & A045 & & & & & & & & & & & 0.075 & 0.078 & 0.077 & 0.077 & 0.002 \\
\hline & A046 & 0 & 0.1 & 0 & 0.0333 & 0.0577 & 0 & 0 & 0 & 0.0000 & 0.0000 & & & & & \\
\hline & A 047 & 0.01 & 0.01 & 0.01 & 0.0100 & 0.0000 & 0.02 & 0.02 & 0.02 & 0.0200 & 0.0000 & & & & & \\
\hline & A048 & 0.075 & 0.076 & 0.095 & 0.0820 & 0.0113 & 0.115 & 0.114 & 0.114 & 0.1143 & 0.0006 & & & & & \\
\hline & A 050 & 0.134 & 0.135 & 0.134 & 0.1343 & 0.0006 & 0.096 & 0.093 & 0.0946 & 0.0945 & 0.0015 & & & & & \\
\hline & A052 & & & & & & & & & & & & & & & \\
\hline & A053 & & & & & & & & & & & & & & & \\
\hline & $\mathrm{A} 055$ & 0.1315 & 0.1315 & 0.1348 & 0.1326 & 0.0019 & 0.1662 & 0.1602 & 0.161 & 0.1625 & 0.0033 & & & & & \\
\hline 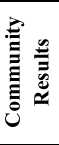 & & $\begin{array}{l}\text { Consensu } \\
\text { Consensu } \\
\text { Maximum } \\
\text { Minimum } \\
\mathrm{N}\end{array}$ & $\begin{array}{l}\text { is Mean } \\
\text { s Standard }\end{array}$ & Deviation & $\begin{array}{c}0.1349 \\
0.0038 \\
0.7000 \\
0.0000 \\
48 \\
\end{array}$ & & $\begin{array}{l}\text { Consensu } \\
\text { Consensu } \\
\text { Maximum } \\
\text { Minimum } \\
\mathrm{N}\end{array}$ & $\begin{array}{l}\text { Is Mean } \\
\text { standard }\end{array}$ & Deviation & $\begin{array}{c}0.1686 \\
0.0057 \\
0.8000 \\
0.0000 \\
54 \\
\end{array}$ & & $\begin{array}{l}\text { Consensu } \\
\text { Consensu } \\
\text { Maximun } \\
\text { Minimum } \\
\mathrm{N}\end{array}$ & $\begin{array}{l}\text { Mean } \\
\text { Standard }\end{array}$ & Deviation & $\begin{array}{c}0.134 \\
0.016 \\
0.1933 \\
0.0667 \\
11 \\
\end{array}$ & \\
\hline
\end{tabular}




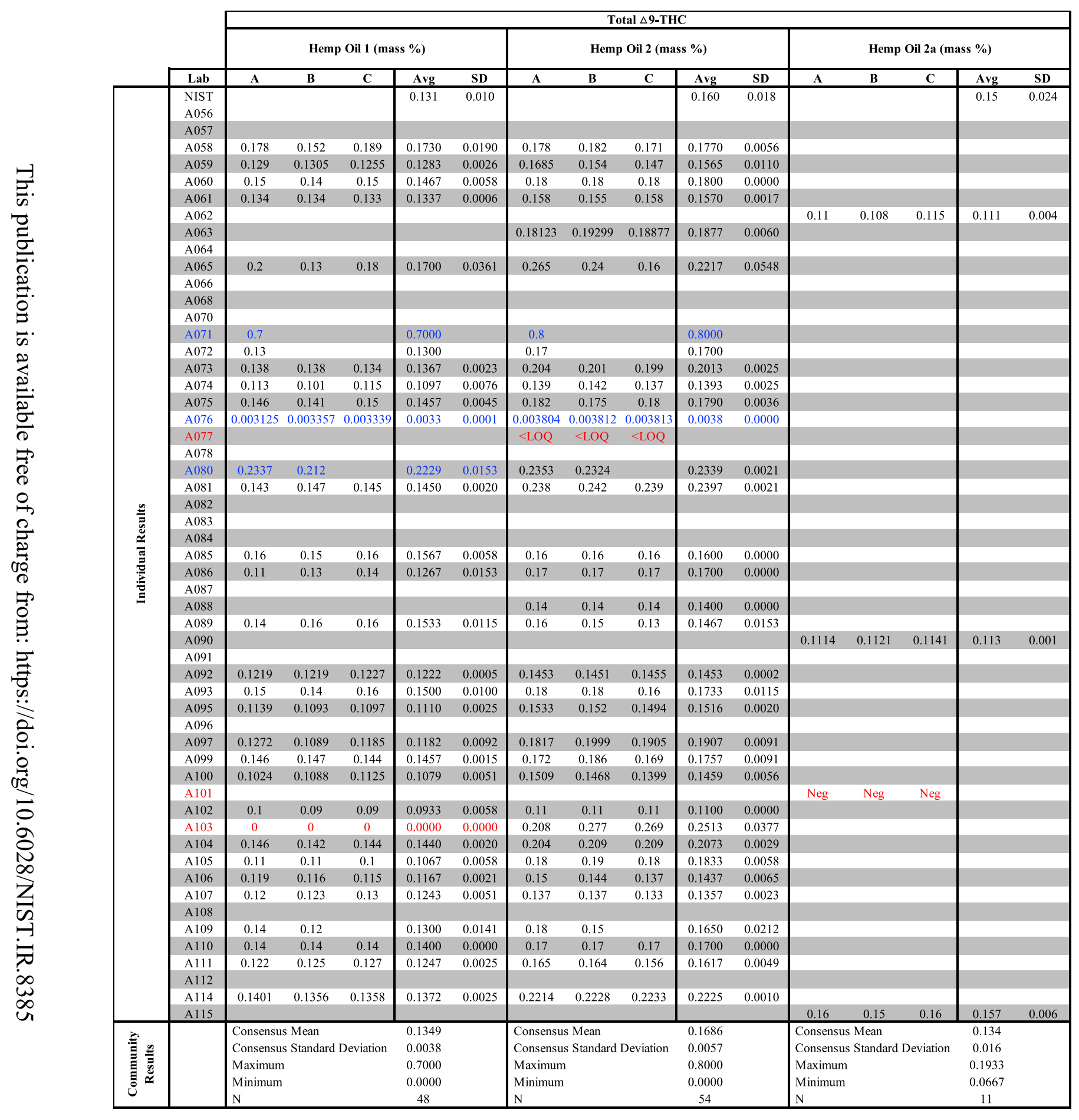




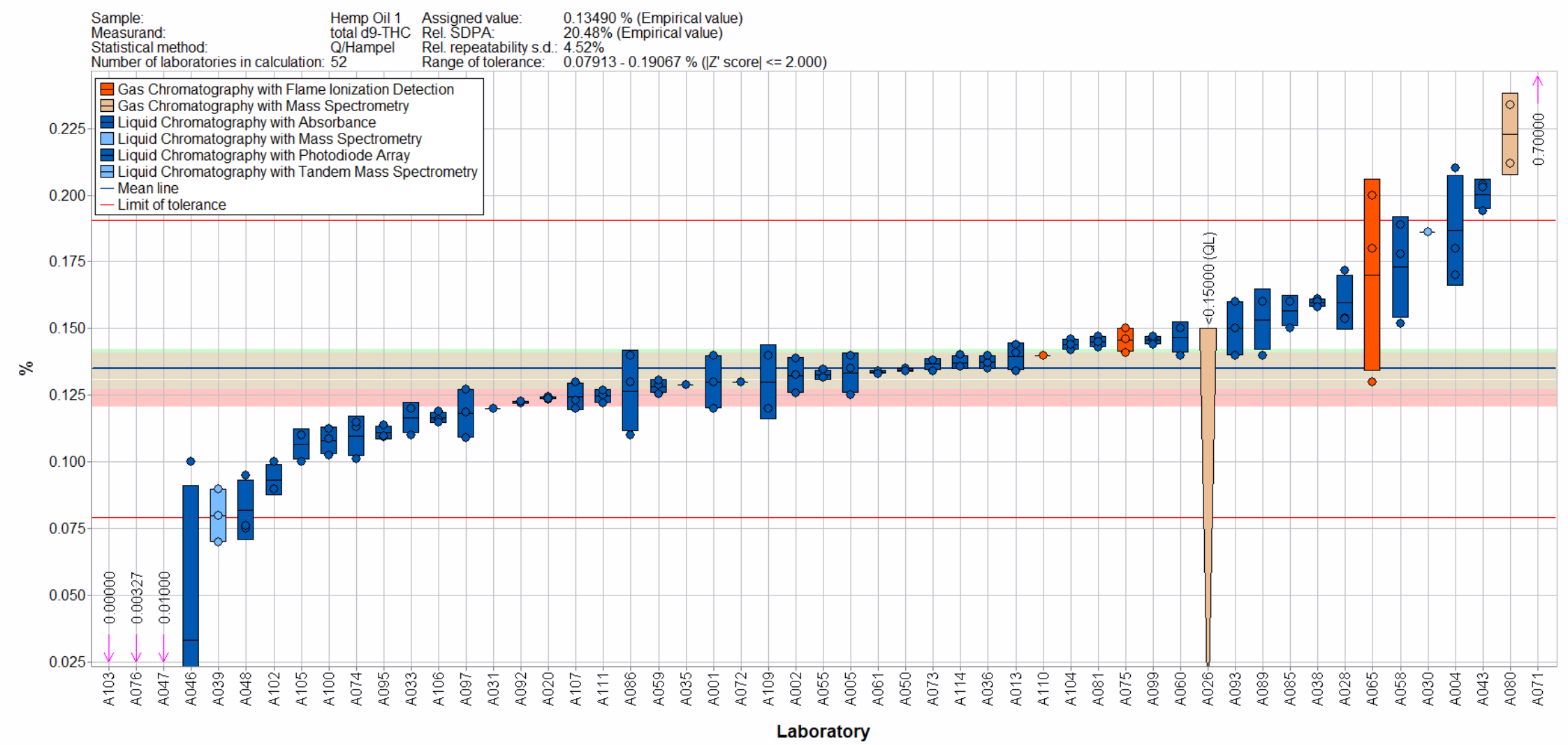

Figure 2-13. Total THC in Hemp Oil 1 (data summary view - analytical method). In this view, individual laboratory data are plotted (circles) with the individual laboratory standard deviation (rectangle). The color of the data point represents the analytical method employed. The solid blue line represents the consensus mean, and the green shaded region represents the $95 \%$ confidence interval for the consensus mean. The solid red lines represent the consensus range of tolerance, calculated as the values above and below the consensus mean that result in an acceptable $Z_{\text {comm }}^{\prime}$ score, $\left|Z_{\text {comm }}^{\prime}\right| \leq 2$. The red shaded region represents the NIST range of tolerance, which encompasses the target value bounded by twice its uncertainty ( $\left.U_{\mathrm{NIST}}\right)$ and represents the range that results in an acceptable $Z_{\mathrm{NIST}}$ score, $\left|Z_{\mathrm{NIST}}\right| \leq 2$. The shaded beige region represents the overlapping of the $95 \%$ confidence interval for the consensus mean (green region) and the NIST range of tolerance (red region). The downward triangle represents data reported as a threshold or LOQ value. 


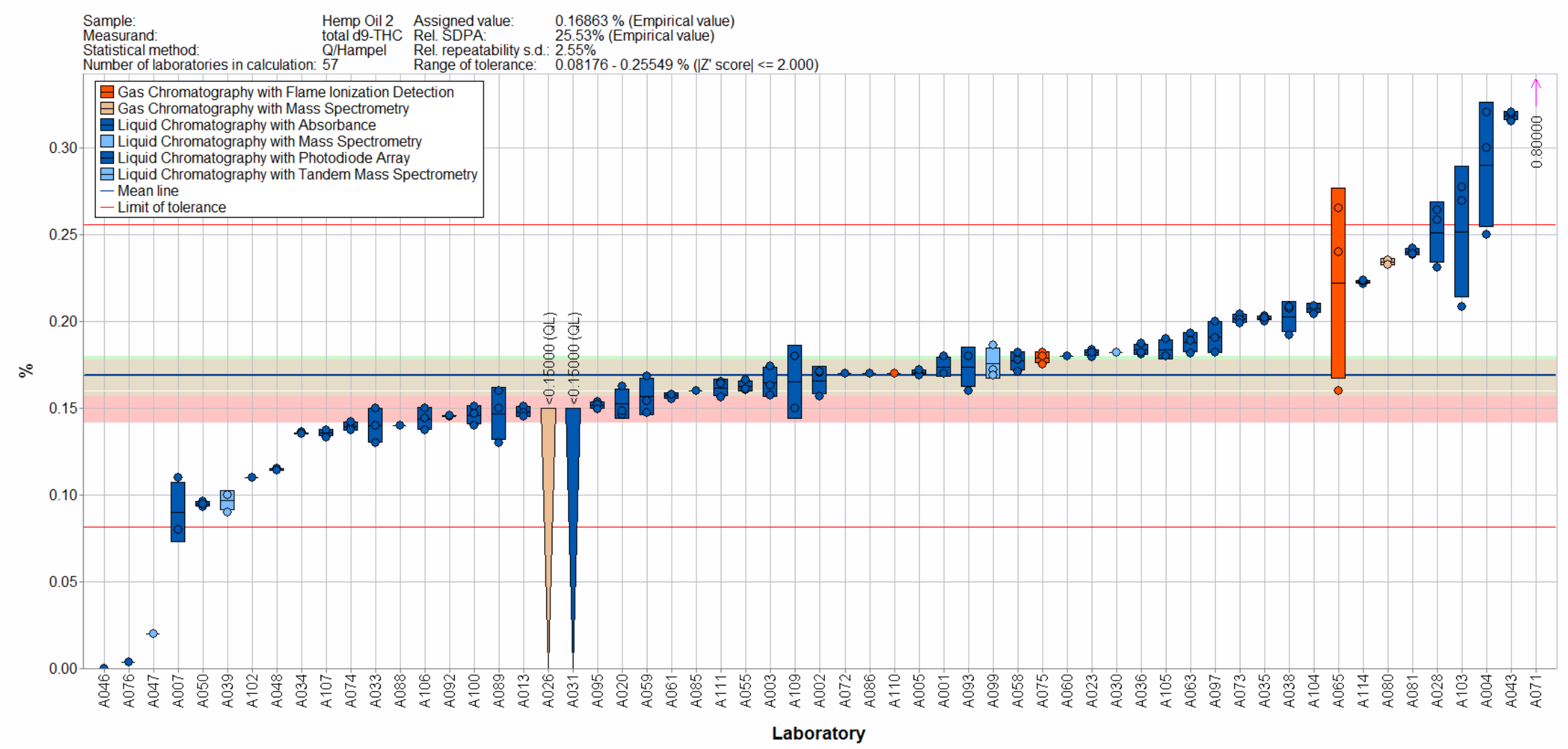

Figure 2-14. Total THC in Hemp Oil 2 (data summary view - analytical method). In this view, individual laboratory data are plotted (circles) with the individual laboratory standard deviation (rectangle). The color of the data point represents the analytical method employed. The solid blue line represents the consensus mean, and the green shaded region represents the $95 \%$ confidence interval for the consensus mean. The solid red lines represent the consensus range of tolerance, calculated as the values above and below the consensus mean that result in an acceptable $Z_{\text {comm }}^{\prime}$ score, $\left|Z_{\text {comm }}^{\prime}\right| \leq 2$. The red shaded region represents the NIST range of tolerance, which encompasses the target value bounded by twice its uncertainty ( $\left.U_{\mathrm{NIST}}\right)$ and represents the range that results in an acceptable $Z_{\mathrm{NIST}}$ score, $\left|Z_{\mathrm{NIST}}\right| \leq 2$. The shaded beige region represents the overlapping of the $95 \%$ confidence interval for the consensus mean (green region) and the NIST range of tolerance (red region). The downward triangle represents data reported as a threshold or LOQ value. 


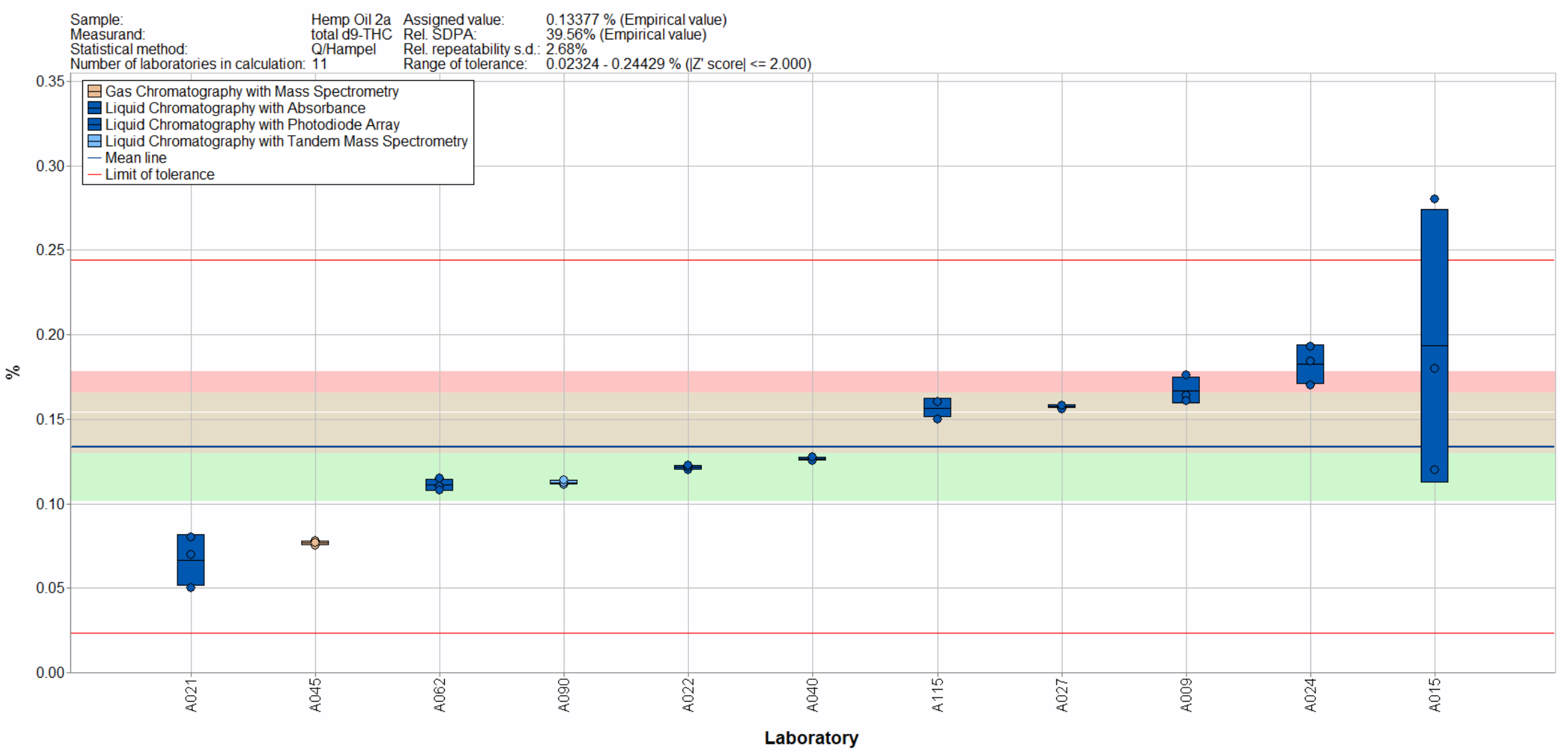

Figure 2-15. Total THC in Hemp Oil 2a (data summary view - analytical method). In this view, individual laboratory data are plotted (circles) with the individual laboratory standard deviation (rectangle). The color of the data point represents the analytical method employed. The solid blue line represents the consensus mean, and the green shaded region represents the $95 \%$ confidence interval for the consensus mean. The solid red lines represent the consensus range of tolerance, calculated as the values above and below the consensus mean that result in an acceptable $Z_{\text {comm }}^{\prime}$ score, $\left|Z_{\text {comm }}^{\prime}\right| \leq 2$. The red shaded region represents the NIST range of tolerance, which encompasses the target value bounded by twice its uncertainty ( $\left.U_{\mathrm{NIST}}\right)$ and represents the range that results in an acceptable $Z_{\mathrm{NIST}}$ score, $\left|Z_{\mathrm{NIST}}\right| \leq 2$. The shaded beige region represents the overlapping of the $95 \%$ confidence interval for the consensus mean (green region) and the NIST range of tolerance (red region). The downward triangle represents data reported as a threshold or LOQ value. 


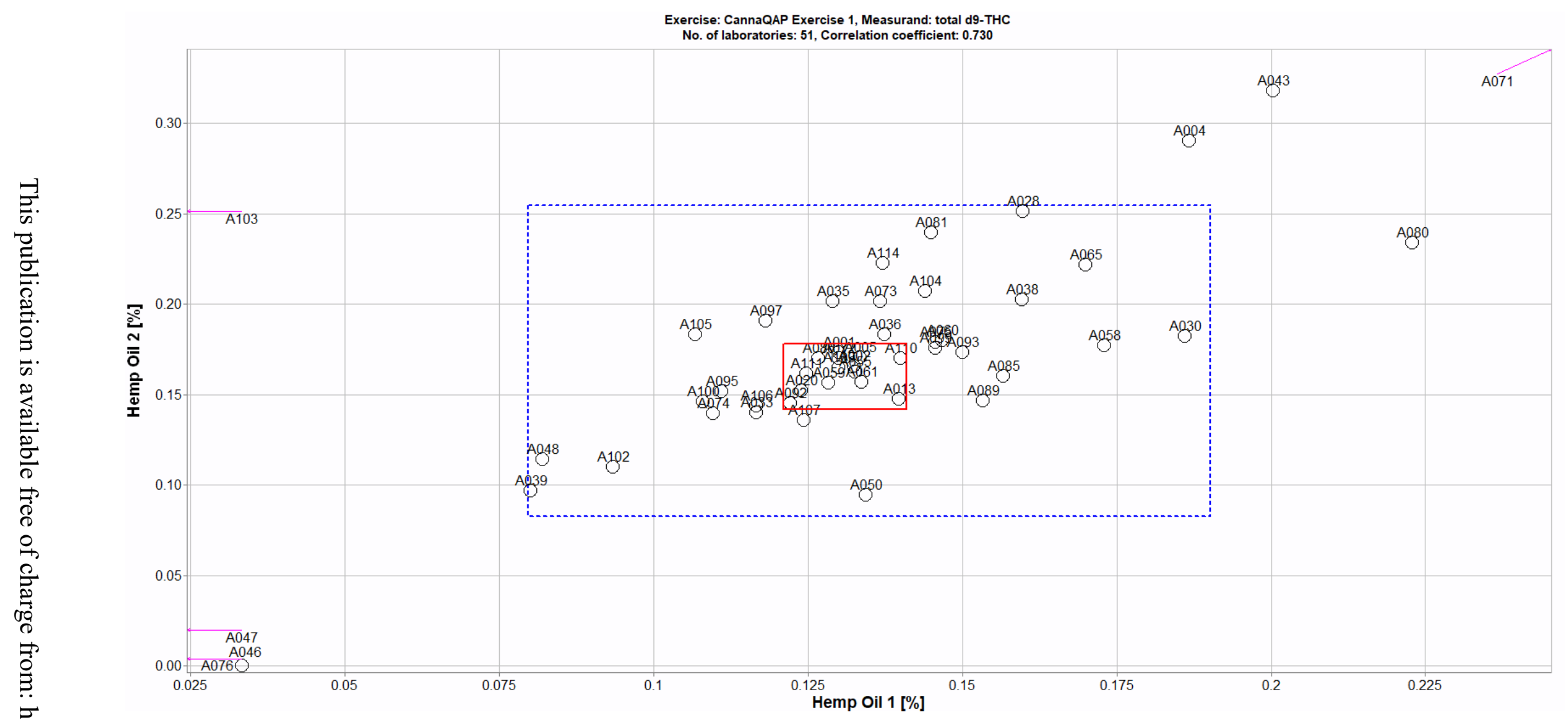

Figure 2-16. Laboratory means for total THC in Hemp Oil 1 and Hemp Oil 2 (sample/sample comparison view). In this view, the individual laboratory mean for one sample (Hemp Oil 1) is compared to the individual laboratory mean for a second sample (Hemp Oil 2). The solid red box represents the NIST range of tolerance for the two samples, Hemp Oil 1 (x-axis) and Hemp Oil 2 (y-axis), which encompasses the target values bounded by their uncertainties ( $U_{\text {NIST }}$ ) and represents the range that results in an acceptable $Z_{\text {NIST }}$ score, $\left|Z_{\mathrm{NIST}}\right| \leq 2$. The dotted blue box represents the consensus range of tolerance for Hemp Oil 1 (x-axis) and Hemp Oil 2 (y-axis), calculated as the values above and below the consensus means that result in an acceptable $Z_{\text {comm }}^{\prime}$ score, $\left|Z_{\text {comm }}^{\prime}\right| \leq 2$. 


\section{SECTION 3: CBD, CBDA, AND TOTAL CBD}

Study Overview

CBD is the primary cannabinoid found in hemp oils, which has attracted significant interest due to numerous purported beneficial health effects along with its safety and tolerability profile in humans. ${ }^{6}$ The first CBD drug, Epidiolex, was approved by the US FDA in 2018 to treat two rare forms of epilepsy. ${ }^{7}$ More recently, the FDA has extended approval in 2020 for treatment of seizures associated with tuberous sclerosis complex in patients 1 year and older. CBD does not exist in Cannabis naturally but is formed following decarboxylation of its acidic form (CBDA) through exposure to heat or light. These decarboxylation steps are commonly used in the production of hemp oils to increase CBD content. Participants in this study were asked to use in-house analytical methods to determine the mass fraction (\%) of CBD, CBDA, and total CBD in three hemp oils. The preparation of these hemp oils included a decarboxylation step resulting in extremely low levels of CBDA and levels of CBD in normal commercial product ranges.

Reporting Statistics

- The enrollment and reporting statistics for CBD, CBDA, and total CBD are described in the table below for each analyte. Reported values may include non-quantitative results (zero or below LOQ).

\begin{tabular}{cccccccc} 
& \multicolumn{2}{c}{ Hemp Oil 1 } & \multicolumn{2}{c}{ Hemp Oil 2 } & \multicolumn{2}{c}{ Hemp Oil 2a } \\
& $\underline{\text { Number of }}$ & $\underline{\underline{\text { Reporting }}}$ & $\underline{\text { Number of }}$ & $\underline{\underline{\text { Reporting }}}$ & $\underline{\underline{\text { Number of }}}$ & $\underline{\underline{\text { Reporting }}}$ \\
$\underline{\text { Analyte }}$ & $\underline{\underline{\text { Participants }}}$ & $\underline{\underline{\text { Results }}}$ & $\underline{\underline{\text { Participants }}}$ & $\underline{\text { Results }}$ & $\underline{\text { Participants }}$ & $\underline{\text { Results }}$ \\
CBD & 77 & $88 \%$ & 90 & $88 \%$ & 19 & $63 \%$ \\
CBDA & 72 & $76 \%$ & 81 & $73 \%$ & 19 & $42 \%$ \\
Total CBD & 68 & $71 \%$ & 75 & $73 \%$ & 19 & $53 \%$
\end{tabular}

- Most laboratories reported using solvent extraction or sample dilution for determination of CBD, CBDA, and total CBD in the three hemp oil samples (see table below). Additional sample preparation details are summarized at the end of the report in Appendix I.

$\begin{array}{lccc}\begin{array}{c}\text { Reported } \\ \text { Preparation Method }\end{array} & \underline{\text { CBD }} & \underline{\text { Percent Reporting }} & \text { Total CBD } \\ \text { Solvent Extraction } & 68.3 & 71.5 & 70.3 \\ \text { Dilution } & 26.7 & 24.1 & 22.7 \\ \text { Other } & 0.6 & 1.5 & 2.3 \\ \text { None } & 1.2 & 0.0 & 0.0 \\ \text { No Response } & 3.1 & 2.9 & 4.7 \\ & & \\ \text { 6 X Lim, T Tan, S Rosli, M Sa'at, S Ali, A Mohamed. PLOS ONE 16(1): 1-22 (2021) } & \\ \text { https:///oi.org/10.1371/journal.pone.0245471. } \\ \text { https://www.fda.gov/news-events/press-announcements/fda-approves-first-drug-comprised-active-ingredient- } \\ \text { derived-marijuana-treat-rare-severe-forms. }\end{array}$


- Most laboratories reported using LC-PDA or LC-UV for the determination of CBD, CBDA, and total CBD (see table below). Additional method details are summarized at the end of the report in Appendix I.

$\underline{\text { Reported }}$

Analytical Method

LC-PDA

LC-UV

LC-MS

LC-MS/MS

GC-FID

GC-MS

Other
$\underline{\mathrm{CBD}}$

26.1

1.9

3.1

3.1

3.1

0.0

\section{Percent Reporting}

CBDA

62.8

31.4

2.2

3.6

0.0

0.0

0.0 $\underline{\text { Total CBD }}$

66.4

25.8

1.6

2.3

3.1

0.0

0.8

Study Results

$C B D$

- The mass fractions (\%) for CBD were determined by NIST using LC-PDA as described in Section 2 and are summarized in Table 3-1. These NIST values are used as the target means and ranges summarized Table 3-2 for comparison to the participant results.

- The target and consensus means and ranges are summarized for CBD via different analytical methods in Figure 3-1, Figure 3-2, and Figure 3-3, which include data from laboratories submitting two or three results for CBD. Data from participants submitting only one measurement were included in Table 3-2 but were not included in the calculation of consensus statistics. $^{2}$

- For CBD in Hemp Oil 1, the consensus range was based on quantitative results from 68 laboratories and completely overlaps the target range (Figure 3-1).

- The individual laboratory means from 31 laboratories (46\% of those reporting results) were outside the NIST range of tolerance for CBD in Hemp Oil 1.

- The individual laboratory means from 9 laboratories (13\% of those reporting results) were outside the acceptable $Z_{\text {comm }}^{\prime}$ score for CBD in Hemp Oil 1.

- No results were reported using thresholds or LOQs for CBD in Hemp Oil 1.

- For CBD in Hemp Oil 2, the consensus range was based on quantitative results from 79 laboratories and completely overlaps the target range (Figure 3-2).

- The individual laboratory means from 15 laboratories (19\% of those reporting results) were outside the NIST range of tolerance for CBD in Hemp Oil 2.

- The individual laboratory means from 9 laboratories (11\% of those reporting results) were outside the acceptable $Z_{\text {comm }}^{\prime}$ score for CBD in Hemp Oil 2.

- No results were reported using thresholds or LOQs for CBD in Hemp Oil 2.

- For CBD in Hemp Oil 2a, the consensus range was based on quantitative results from 11 laboratories and does not overlap the target range (Figure 3-3).

- The individual laboratory means from 11 laboratories (100\% of those reporting results) were outside the NIST range of tolerance for CBD in Hemp Oil 2a. 
- All individual laboratory means were within the acceptable $Z_{\text {comm }}^{\prime}$ score for CBD in Hemp Oil 2a.

- No results were reported using thresholds or LOQs for CBD in Hemp Oil 2.

- A comparison of individual laboratory means for $\Delta^{9}$-THC in Hemp Oil 1 and Hemp Oil 2 is summarized in Figure 3-4 for laboratories who reported results for both samples.

$C B D A$

- No target means or ranges were provided in Table 3-1 for CBDA in the three hemp oils.

- The consensus means and ranges for CBDA are based on quantitative data from 38 laboratories (Figure 3-5), 34 laboratories (Figure 3-6), and 4 laboratories (Figure 3-7) for Hemp Oil 1, Hemp Oil 2, and Hemp Oil 2a, respectively. Data from participants submitting only one measurement were included in Table 3-3 but were not included in the calculation of consensus statistics. $^{2}$

- A comparison of individual laboratory means for CBDA in Hemp Oil 1 and Hemp Oil 2 is summarized in Figure 3-8 for laboratories who reported results for both samples.

\section{Total CBD}

- The mass fractions (\%) for total CBD in the hemp oil samples were determined by NIST using LC-PDA as described in Section 1 and are summarized in Table 3-1. These NIST values are used as the target means and ranges summarized in Table 3-4 for comparison to the participant results.

- The target and consensus means and ranges are summarized for total CBD via different analytical methods in Figure 3-9, Figure 3-10, and Figure 3-11, which include data from laboratories submitting two or three measurements for total CBD. Data from participants submitting only one measurement were included in Table 3-4 but were not included in the calculation of consensus statistics. ${ }^{2}$

- For total CBD in Hemp Oil 1, the consensus range was based on quantitative results from 48 laboratories and is completely within the target range (Figure 3-9).

- The individual laboratory means from 23 laboratories (48\% of those reporting results) were outside the NIST range of tolerance for total CBD in Hemp Oil 1.

- The individual laboratory means from 6 laboratories (13\% of those reporting results) were outside the acceptable $Z_{\text {comm }}^{\prime}$ score for total CBD in Hemp Oil 1.

- No results were reported using thresholds or LOQs for CBD in Hemp Oil 1.

- For total CBD in Hemp Oil 2, the consensus range was based on quantitative results from 55 laboratories and its completely within the target range (Figure 3-10).

- The individual laboratory means from 11 laboratories (20\% of those reporting results) were outside the NIST range of tolerance for total CBD in Hemp Oil 2.

- The individual laboratory means from 5 laboratories ( $9 \%$ of those reporting results) were outside the acceptable $Z_{\text {comm }}^{\prime}$ score for total CBD in Hemp Oil 2.

- No results were reported using thresholds or LOQs for CBD in Hemp Oil 2.

- For total CBD in Hemp Oil 2a, the consensus range was based on quantitative results from 10 laboratories and does not overlap the target range (Figure 3-11).

- The individual laboratory means from 10 laboratories (100\% of those reporting results) were outside the NIST range of tolerance for total CBD in Hemp Oil 2a. 
- No individual laboratory means were outside the acceptable $Z_{\text {comm }}^{\prime}$ score for total CBD in Hemp Oil 2a.

- No results were reported using thresholds or LOQs for CBD in Hemp Oil 2a.

- A comparison of individual laboratory means for total CBD in Hemp Oil 1 and Hemp Oil 2 is summarized in Figure 3-12 for laboratories who reported results for both samples.

\section{Overall}

- The between-laboratory variabilities for determination of CBD, CBDA, and total CBD in the hemp oil samples are shown in the table below.

Between-Laboratory Variability (\% RSD)

\begin{tabular}{|c|c|c|c|}
\hline Analyte & $\underline{\text { Hemp Oil } 1}$ & Hemp Oil 2 & $\underline{\text { Hemp Oil 2a }}$ \\
\hline CBD & 1.0 & 0.9 & 8.5 \\
\hline CBDA & 9.0 & 26.0 & 78.2 \\
\hline Total CBD & 1.1 & 1.0 & 9.4 \\
\hline
\end{tabular}

Study Discussion and Technical Recommendations

The following recommendations are based on results obtained from the participants in this study.

$C B D$

- Approximately $15 \%$ of the laboratories reporting results for CBD provided values outside the consensus ranges for both Hemp Oil 1 and Hemp Oil 2 (Figure 3-4).

- Laboratories reporting results below the consensus mean in Hemp Oil 1 also reported results below the consensus mean for Hemp Oil 2. The reverse is also observed for laboratories reporting values above the consensus mean in both samples. Trends of this type often indicate a calibration bias.

- The between-laboratory variability for CBD was higher in Hemp Oil 2a (9.4\%) than Hemp Oil 1 or Hemp Oil $2(\approx 1 \%)$. The variability of individual laboratory means was slightly higher for CBD in Hemp Oil 1 (2.6 \%) and Hemp Oil 2 (2.2\%) in comparison to Hemp Oil 2a (1.4 \%).

- Hemp Oil 2a was prepared through a methanol/ethanol extraction of Hemp Oil 2 at NIST. The additional processing of Hemp Oil 2a was expected to result in minimal variability both within and among participating laboratories.

- The elevated level of variability may be an artifact of significantly fewer laboratories reporting results for Hemp Oil 2a (11) compared to Hemp Oil 1 (68) and Hemp Oil 2 (79).

- No additional trends were observed for the sample preparation, analytical methods, and/or analytical parameters reported for CBD in the three hemp oil samples.

$C B D A$

- Approximately $15 \%$ of the laboratories reporting results for CBDA provided values outside the consensus ranges for both Hemp Oil 1 and Hemp Oil 2 (Figure 3-8).

- Laboratories reporting results above the consensus mean in Hemp Oil 1 did not necessarily report results above the consensus mean for Hemp Oil 2. Trends of this type often represent potential sample interferences and miss identifications due to levels of CBDA being at or below participants LOQs. 
- No laboratories reported results below the consensus mean in Hemp Oil 1 or Hemp Oil 2 for CBDA.

- Most laboratories reported that CBDA was present in the samples at or below their LOQ (nonzero values). The low levels resulted in large consensus ranges and between-laboratory variabilities $(9.0 \%$ to $78.2 \%)$.

- Approximately $6 \%$ of the laboratories reporting results used LC-MS or LC-MS/MS methods with all having low enough LOQs to determine CBDA at the consensus levels in Hemp Oil 1, Hemp Oil 2, and Hemp Oil 2a.

- Approximately $94 \%$ of the laboratories reporting results used LC-UV or LC-PDA methods with only $50 \%, 38 \%$, and $29 \%$ of these laboratories with low enough LOQs to determine CBDA at the consensus levels in Hemp Oil 1, Hemp Oil 2, and Hemp Oil 2a, respectively.

- No additional trends were observed for the sample preparation, analytical methods, and/or analytical parameters reported for CBDA in the three hemp oil samples.

\section{Total CBD}

- Approximately $15 \%$ of the laboratories reporting results for total CBD provided values outside the consensus range for both Hemp Oil 1 and Hemp Oil 2 (Figure 3-12).

- Laboratories reporting results below the consensus mean in Hemp Oil 1 also reported results below the consensus mean for Hemp Oil 2. The reverse is also observed for laboratories reporting values above the consensus mean in both samples. Trends of this type often indicate a calibration bias.

- The between-laboratory variability for total CBD was higher in Hemp Oil $2 \mathrm{a}(\approx 9 \%)$ than Hemp Oil 1 or Hemp Oil $2(\approx 1 \%)$. The variability of individual mean laboratory means was slightly higher for total CBD in Hemp Oil $1(\approx 2.4 \%)$ and Hemp Oil $2(\approx 2.2 \%)$ in comparison to Hemp Oil $2 \mathrm{a}(\approx 1.4 \%)$.

- Hemp Oil 2a was prepared through a methanol/ethanol extraction of Hemp Oil 2 at NIST. The additional processing of Hemp Oil $2 \mathrm{a}$ was expected to result in minimal variability both within and among participating laboratories.

- The elevated level of variability may be an artifact of significantly fewer laboratories reporting results for Hemp Oil 2a (10) compared to Hemp Oil 1 (48) and Hemp Oil 2 (55).

- No additional trends were observed for the sample preparation, analytical methods, and/or analytical parameters reported for total CBD in the three hemp oil samples.

\section{Overall}

- Proper storage conditions are important for hemp oils because CBDA can readily convert to CBD when stored at elevated or room temperatures.

- Participants were asked to store the samples under controlled refrigeration $\left(\approx 4{ }^{\circ} \mathrm{C}\right)$.

- Laboratories should perform in-house evaluations of their storage conditions through stability test of their hemp oils at various environmental factors such as temperature, humidity, and light exposure. ${ }^{5}$

- Laboratories should make total CBD determinations via experimentally converting CBDA to $\mathrm{CBD}$ (using elevated temperature or specific chemical reagents) or via calculation of total CBD from the sum of measured CBD and CBDA in the sample using the equation below.

$$
\text { Total } C B D=\text { mass } \% C B D+(0.877 \times \operatorname{mass} \% C B D A)
$$


- Over 100 cannabinoids have been identified in Cannabis plant samples with similarities in structure and molecular mass. As a result, chromatographic peak identity should always be confirmed using appropriate reference spectra of pure standards for cannabinoids and is particularly important for cannabinoids that are present at levels close to the method LOQ. Analytical methods should be able to clearly distinguish between cannabinoids.

- The use of appropriate calibration materials and quality assurance samples to establish that a method is in control and being performed correctly may reduce the likelihood of outlying data. Quality assurance samples can be commercially available reference materials (CRMs, SRMs, or RMs) or materials prepared in-house.

- Measurement results should be reported accurately.

- Reported values should be the mass fraction (\%) of the CBD, CBDA, and total CBD in the three hemp oil samples. Participants who reported values based on a volume fraction (\%) for Hemp Oil 2 or Hemp Oil 2a should use the density of the sample for the conversion to mass fraction. Hemp Oil 1 was too viscous to prepare dilutions by volume.

- Zero is not a quantity that can be measured. If values are below LOQ, results should be reported as such. A more appropriate result would be to report that a value is below the LOQ (e.g., " $<0.02 ")$.

- Laboratories reporting results based on a certain threshold should enter the numerical threshold (e.g., " $<1$ ").

- Laboratories reporting results flagged as outliers should check for calculation errors when preliminary data tables are sent for inspection. One example is to confirm that factors for all dilutions have been properly tabulated or that results are reported in the requested units. 
Table 3-1. Individualized data summary table (NIST) for CBD, CBDA, and total CBD in hemp oils.

\section{National Institute of Standards and Technology}

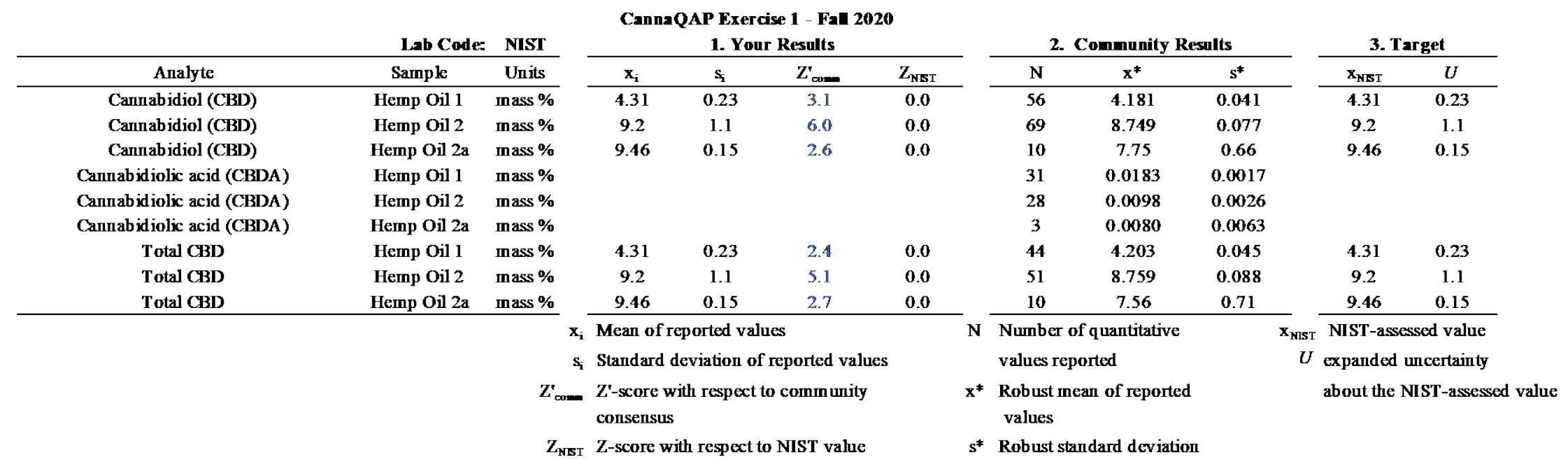


Table 3-2. Data summary table for CBD in hemp oils. Data highlighted in red have been flagged as a data entry of zero or results that include text (e.g., "<LOQ" or "present"). Data highlighted in blue have been identified as outside the consensus tolerance limits and would be estimated to result in an unacceptable $Z_{\text {comm }}^{\prime}$ score, $\left|Z_{\text {comm }}^{\prime}\right| \geq 2$. Note: This table spans two pages; the NIST values and consensus values are included on both pages for convenience.

\begin{tabular}{|c|c|c|c|c|c|c|c|c|c|c|c|c|c|c|c|c|}
\hline & \multirow[b]{3}{*}{ Lab } & \multicolumn{15}{|c|}{ Cannabidiol (CHI) } \\
\hline & & \multicolumn{5}{|c|}{ He=p Oill (-ass \%) } & \multicolumn{5}{|c|}{ Henp 012 (mass 96) } & \multicolumn{5}{|c|}{ Henp ON 2a (-ass \%) } \\
\hline & & $\mathbf{A}$ & $\mathbf{B}$ & c & Avg & SD & $\mathbf{A}$ & $\mathbf{B}$ & $\mathbf{C}$ & Avg & SD & $\mathbf{A}$ & $\mathbf{B}$ & $\mathbf{c}$ & Avg & SD \\
\hline \multirow{52}{*}{ 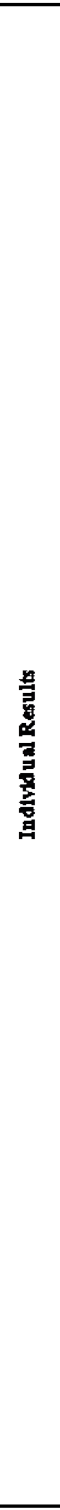 } & NIST & & & & 431 & 0.23 & & & & 92 & 1.1 & & & & 9.46 & 0.15 \\
\hline & A 001 & 4.06 & 4.08 & 4.04 & 4.060 & 0.020 & 8.35 & 8.35 & 8.38 & 8.360 & 0.017 & & & & & \\
\hline & A002 & 432135 & 4.37755 & 424454 & 4314 & 0067 & 9214958 & 8.713401 & 9.708629 & 9212 & 0498 & & & & & \\
\hline & A 003 & & & & & & 9.354 & 9.659 & 9.161 & 9.391 & 0.251 & & & & & \\
\hline & A004 & 3.12 & 298 & 3.1 & 3.067 & 0.076 & 624 & 6.33 & 6.12 & 6230 & 0.105 & & & & & \\
\hline & $\mathrm{A} 005$ & 4.42 & 4.24 & 4.61 & 4.423 & 0.185 & 9.23 & 9.22 & 9.28 & 9.243 & 0.032 & & & & & \\
\hline & A006 & 4.65 & & & 4.650 & & 952 & & & 9520 & & & & & & \\
\hline & A 007 & 1.98 & 1.87 & 1.9 & 1.917 & 0.057 & 8.38 & 8.7 & 8.55 & 8.543 & 0.160 & & & & & \\
\hline & A008 & 435 & & & 4350 & & & & & & & & & & & \\
\hline & A 009 & & & & & & & & & & & 4.516 & 4.53 & 4.533 & 4.53 & 0.01 \\
\hline & A010 & & & & & & & & & & & present & present & present & & \\
\hline & A 011 & & & & & & & & & & & & & & & \\
\hline & A012 & & & & & & 696878 & 6.94137 & 693743 & 6949 & 0.017 & & & & & \\
\hline & $\mathrm{A} 013$ & 4.221 & 4.423 & 4.596 & 4.413 & 0.188 & 8.385 & 9.247 & 9.228 & 8.953 & 0.492 & & & & & \\
\hline & A014 & 422 & & & 4220 & & 923 & & & 9230 & & & & & & \\
\hline & A 015 & & & & & & & & & & & 10.14 & 10.48 & 10.23 & 10.28 & 0.18 \\
\hline & A016 & & & & & & & & & & & & & & & \\
\hline & A 017 & 4.45 & & & 4.450 & & 8.88 & & & 8.880 & & & & & & \\
\hline & A018 & & & & & & & & & & & & & & & \\
\hline & A 019 & 4.16 & & & 4.160 & & 8.51 & & & 8.510 & & & & & & \\
\hline & A020 & 3982784 & 4.055099 & 398743 & 4.008 & 0.040 & 7539147 & 79206 & 838996 & 7950 & 0426 & & & & & \\
\hline & A 021 & & & & & & & & & & & 7.88 & 8.68 & 8.5 & 8.35 & 0.42 \\
\hline & A022 & & & & & & & & & & & 69829 & 7.046 & 7.0714 & 7.03 & 005 \\
\hline & A 023 & & & & & & 8.7195 & 8.6746 & 8.8788 & 8.758 & 0.107 & & & & & \\
\hline & A024 & & & & & & & & & & & 9267 & 9.129 & 9.281 & 923 & 0.08 \\
\hline & A 025 & & & & & & 9.5 & 10 & 9.6 & 9.700 & 0.265 & & & & & \\
\hline & A026 & 3.7 & 3.8 & 39 & 3.800 & 0.100 & 86 & 8.4 & 8.8 & 8600 & 0200 & & & & & \\
\hline & A 027 & & & & & & & & & & & 8.87 & 8.98 & 8.89 & 8.91 & 0.06 \\
\hline & A028 & 4.4883 & 45036 & 4.4891 & 4.494 & 0.009 & 9.1859 & 92043 & 9.0885 & 9.160 & 0062 & & & & & \\
\hline & A 029 & & & & & & 8.5 & 7.7 & 9 & 8.400 & 0.656 & & & & & \\
\hline & A030 & 5.3 & & & 5300 & & 10.4 & & & 10.400 & & & & & & \\
\hline & A 031 & 4.27 & 4.33 & 4.26 & 4.287 & 0.038 & 8.84 & 8.78 & 8.87 & 8.830 & 0.046 & & & & & \\
\hline & A032 & 3.7 & 3.7 & 32 & 3533 & 0.289 & 8.9 & 89 & 8.8 & 8.867 & 0058 & & & & & \\
\hline & A 033 & 3.97 & 3.77 & 3.9 & 3.880 & 0.101 & 8.21 & 8.21 & 8.22 & 8.213 & 0.006 & & & & & \\
\hline & A034 & & & & & & 9.761 & 9552 & 9.566 & 9.626 & 0.117 & & & & & \\
\hline & $\mathrm{A} 035$ & 4.31 & & & 4.310 & & 8.6 & 8.94 & 8.83 & 8.790 & 0.173 & & & & & \\
\hline & A036 & 429 & 43 & 438 & 4323 & 0.049 & 898 & 89 & 8.93 & 8937 & 0.040 & & & & & \\
\hline & A 037 & 4.2 & 4.22 & 4.11 & 4.177 & 0.059 & 8.811 & 8.811 & 8.665 & 8.762 & 0.084 & & & & & \\
\hline & A038 & 4.11 & 4.16 & 4.15 & 4.140 & 0026 & 857 & 8.49 & 8.49 & 8517 & 0046 & & & & & \\
\hline & A 039 & 4.33 & 4.67 & 5.22 & 4.740 & 0.449 & 10.11 & 10.45 & 9.4 & 9.987 & 0.536 & & & & & \\
\hline & A040 & & & & & & & & & & & 720747 & 7.16035 & 722586 & 720 & 0.03 \\
\hline & A 041 & 4.1 & 4 & 3.9 & 4.000 & 0.100 & 8.3 & 8 & 8.5 & 8.267 & 0.252 & & & & & \\
\hline & A043 & 3902 & 3.879 & 3.835 & 3.872 & 0.034 & 9.043 & 8.489 & 8.822 & 8.785 & 0279 & & & & & \\
\hline & A 044 & & & & & & & & & & & & & & & \\
\hline & A045 & & & & & & & & & & & & & & & \\
\hline & A 046 & 4.1 & 4.2 & 4.3 & 4.200 & 0.100 & 9.12 & 9.2 & 9.18 & 9.167 & 0.042 & & & & & \\
\hline & A050 & 4.19 & 4.19 & 4.19 & 4.190 & 0.000 & 929 & 9.29 & 9.42 & 9333 & 0.075 & & & & & \\
\hline & A 051 & & & & & & & & & & & & & & & \\
\hline & A052 & 4.24 & 4.15 & 422 & 4.203 & 0.047 & 8.75 & 8.47 & 854 & 8587 & 0.146 & & & & & \\
\hline & A 053 & & & & & & & & & & & & & & & \\
\hline & A 054 & 492 & 49 & 4.86 & 4.893 & 0031 & 10.02 & 9.95 & 997 & 9980 & 0036 & & & & & \\
\hline & A 055 & 4.0921 & 4.1763 & 4.0588 & 4.109 & 0.061 & 8.7839 & 8.8859 & 8.327 & 8.666 & 0.298 & & & & & \\
\hline 焉 & & \begin{tabular}{|l|l|} 
Consensus \\
Consensus \\
Maximum \\
Minimum \\
$\mathrm{N}$ \\
\end{tabular} & $\begin{array}{l}\text { S Mean } \\
\text { s Standard I } \\
\end{array}$ & Deviation & $\begin{array}{c}4.182 \\
0.040 \\
20.800 \\
0.105 \\
56 \\
\end{array}$ & & \begin{tabular}{|l} 
Consensus \\
Consensus \\
Maximum \\
Minimum \\
$\mathrm{N}$ \\
\end{tabular} & $\begin{array}{l}\text { is Mean } \\
\text { Is Standard I } \\
\text { a }\end{array}$ & Deviation & $\begin{array}{c}8.750 \\
0.075 \\
43.100 \\
0.066 \\
69 \\
\end{array}$ & & \begin{tabular}{|l|} 
Consensus \\
Consensus \\
Maximum \\
Minimum \\
N \\
\end{tabular} & Mean & Deviation & $\begin{array}{c}7.75 \\
0.66 \\
10.28 \\
453 \\
10\end{array}$ & \\
\hline
\end{tabular}




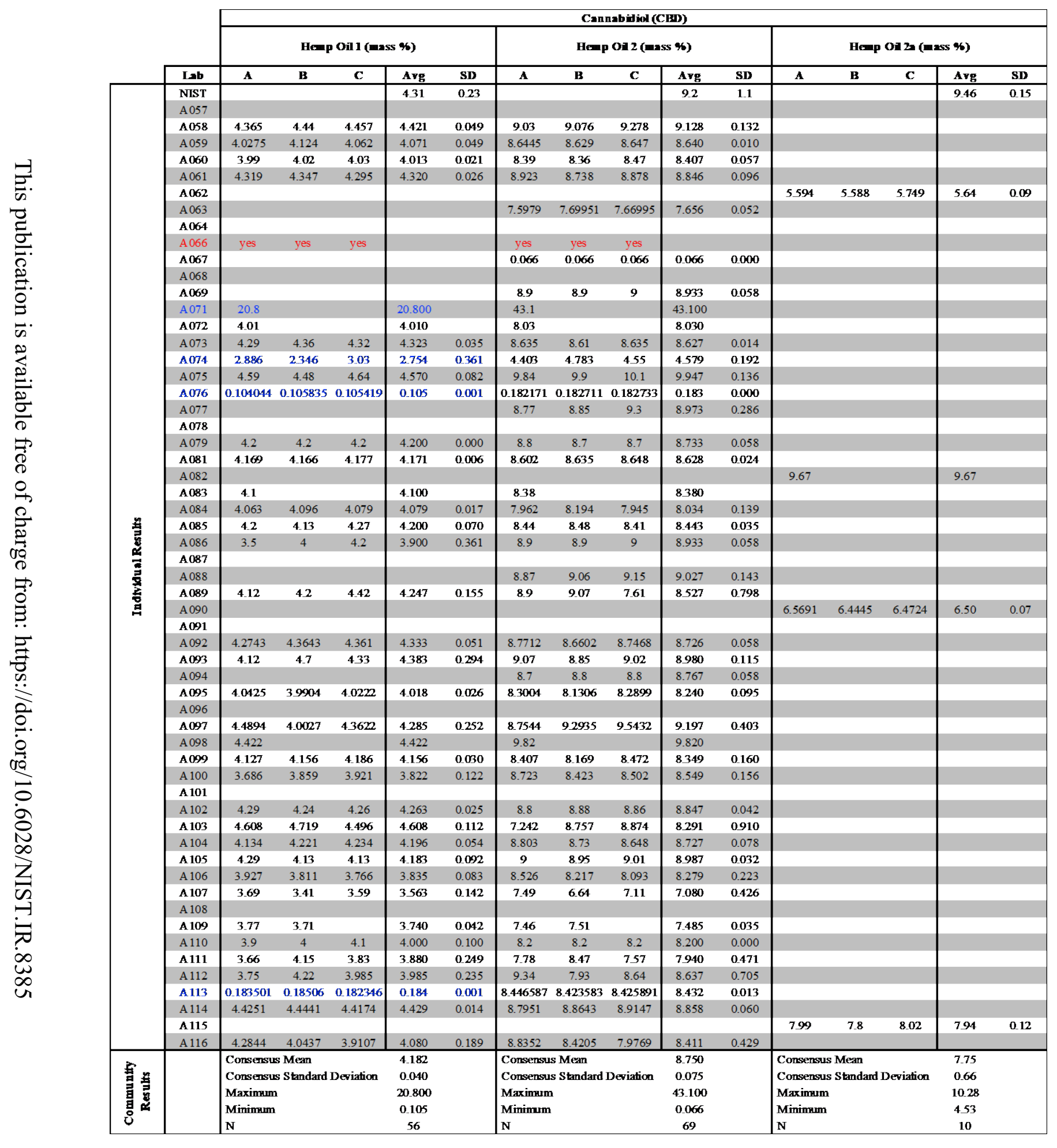




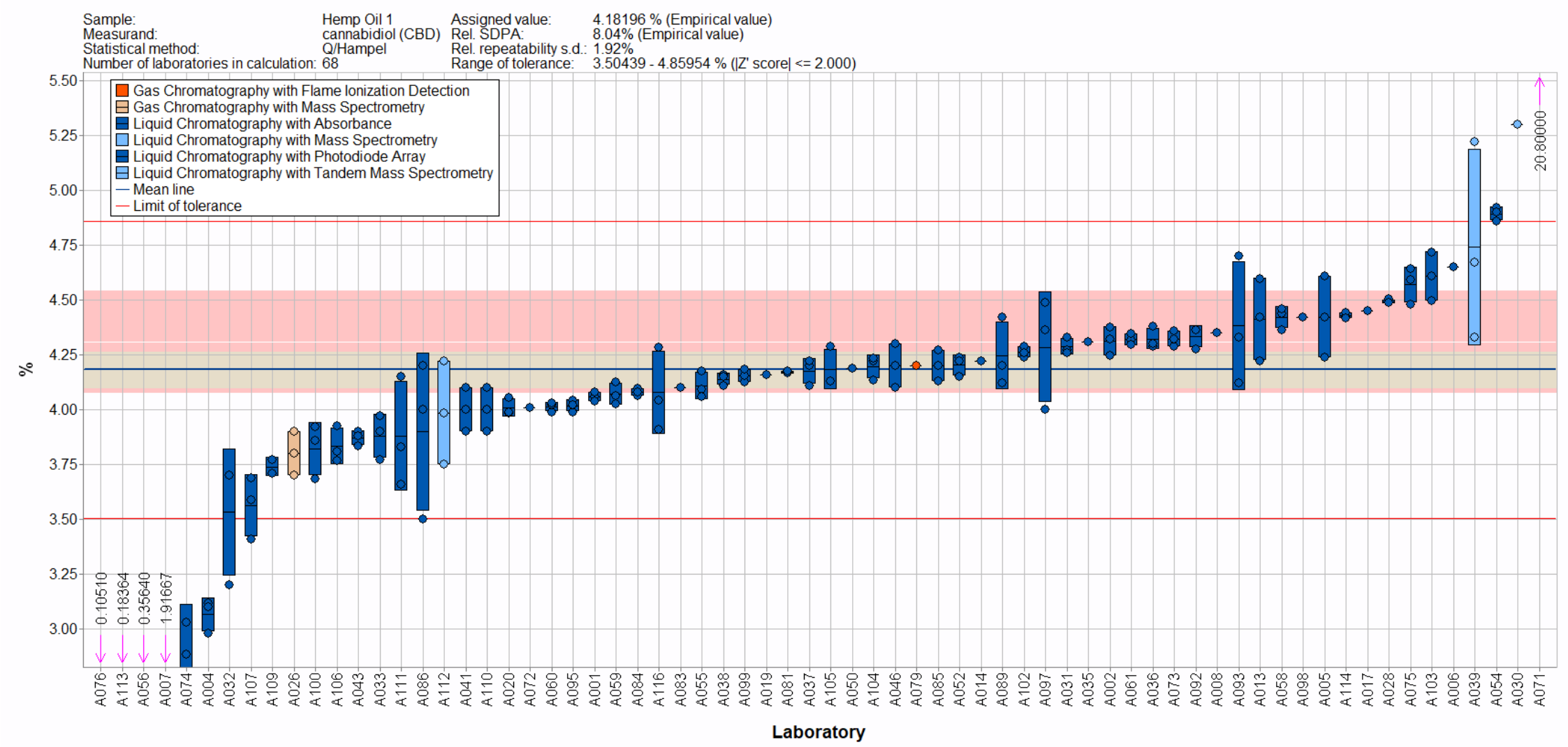

Figure 3-1. CBD in Hemp Oil 1 (data summary view - analytical method). In this view, individual laboratory data are plotted (circles) with the individual laboratory standard deviation (rectangle). The color of the data point represents the analytical method employed. The solid blue line represents the consensus mean, and the green shaded region represents the $95 \%$ confidence interval for the consensus mean. The solid red lines represent the consensus range of tolerance, calculated as the values above and below the consensus mean that result in an acceptable $Z_{\text {comm }}^{\prime}$ score, $\left|Z_{\text {comm }}^{\prime}\right| \leq 2$. The red shaded region represents the NIST range of tolerance, which encompasses the target value bounded by twice its uncertainty ( $U_{\text {NIST }}$ ) and represents the range that results in an acceptable $Z_{\text {NIST }}$ score, $\left|Z_{\text {NIST }}\right| \leq 2$. The shaded beige region represents the overlapping of the $95 \%$ confidence interval for the consensus mean (green region) and the NIST range of tolerance (red region). 


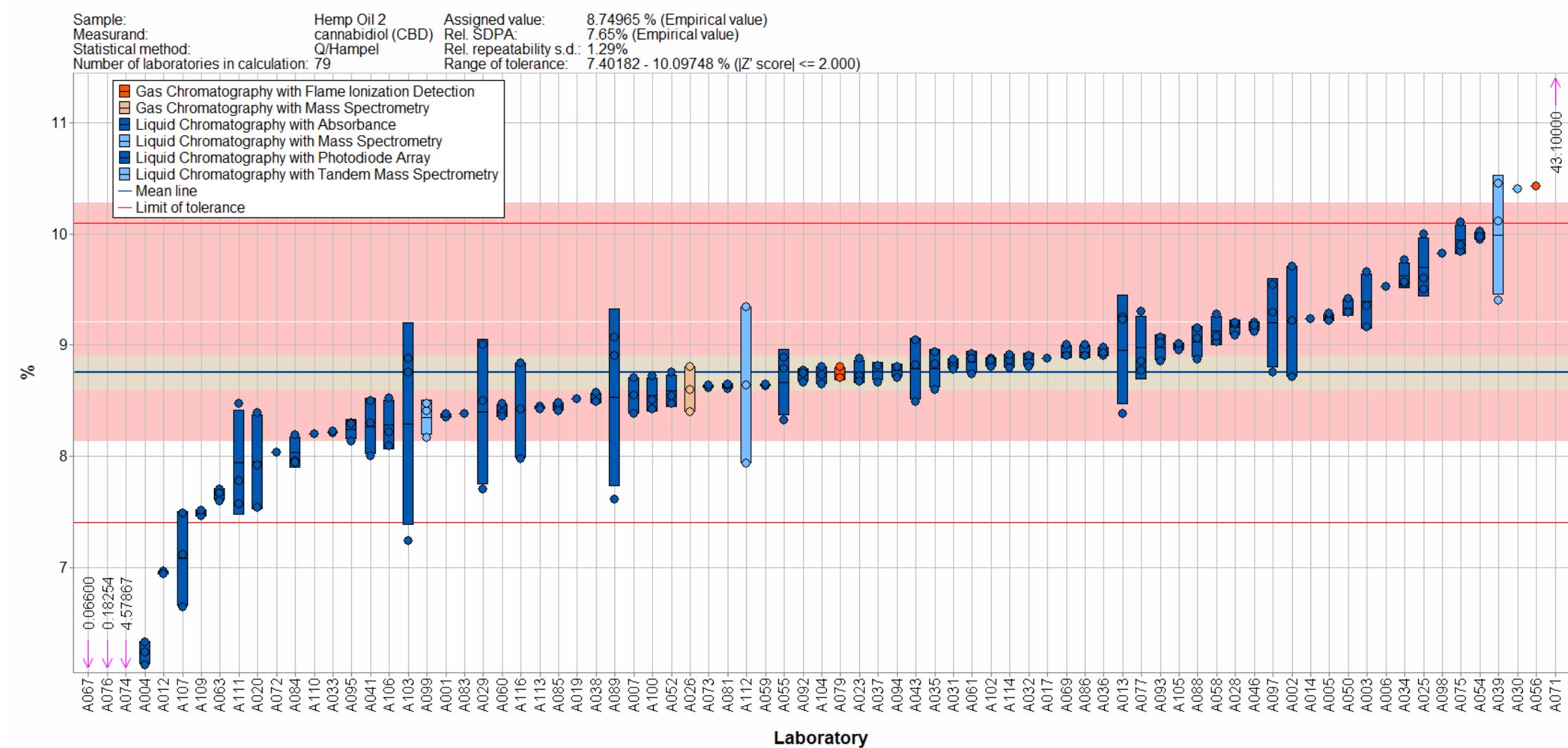

Figure 3-2. CBD in Hemp Oil 2 (data summary view - analytical method). In this view, individual laboratory data are plotted (circles) with the individual laboratory standard deviation (rectangle). The color of the data point represents the analytical method employed. The solid blue line represents the consensus mean, and the green shaded region represents the $95 \%$ confidence interval for the consensus mean. The solid red lines represent the consensus range of tolerance, calculated as the values above and below the consensus mean that result in an acceptable $Z_{\text {comm }}^{\prime}$ score, $\left|Z_{\text {comm }}^{\prime}\right| \leq 2$. The red shaded region represents the NIST range of tolerance, which encompasses the target value bounded by twice its uncertainty $\left(U_{\text {NIST }}\right)$ and represents the range that results in an acceptable $Z_{\text {NIST }}$ score, $\left|Z_{\text {NIST }}\right| \leq 2$. The shaded beige region represents the overlapping of the $95 \%$ confidence interval for the consensus mean (green region) and the NIST range of tolerance (red region). 


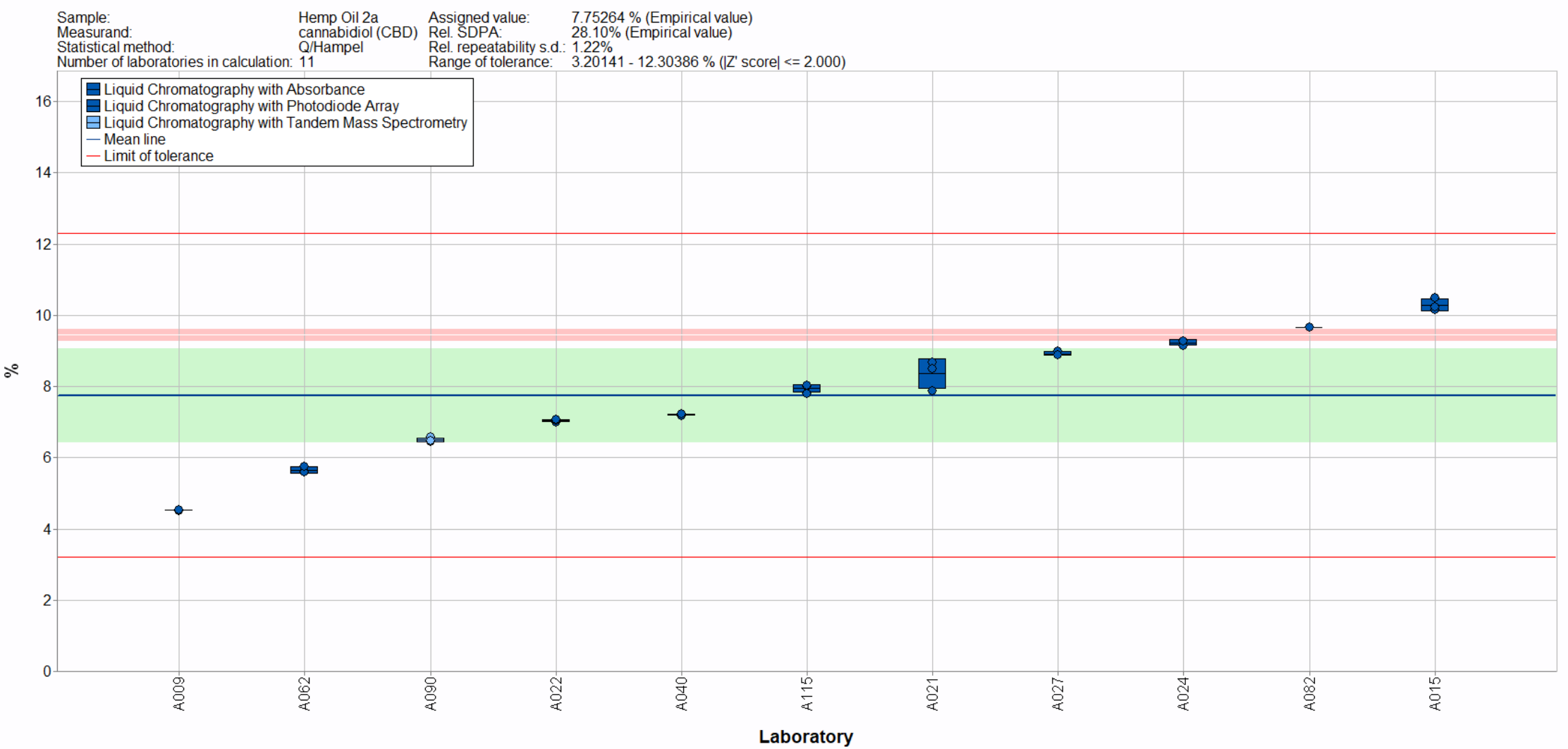

Figure 3-3. CBD in Hemp Oil 2a (data summary view - analytical method). In this view, individual laboratory data are plotted (circles) with the individual laboratory standard deviation (rectangle). The color of the data point represents the analytical method employed. The solid blue line represents the consensus mean, and the green shaded region represents the $95 \%$ confidence interval for the consensus mean. The solid red lines represent the consensus range of tolerance, calculated as the values above and below the consensus mean that result in an acceptable $Z_{\text {comm }}^{\prime}$ score, $\left|Z_{\text {comm }}^{\prime}\right| \leq 2$. The red shaded region represents the NIST range of tolerance, which encompasses the target value bounded by twice its uncertainty $\left(U_{\text {NIST }}\right)$ and represents the range that results in an acceptable $Z_{\text {NIST }}$ score, $\left|Z_{\text {NIST }}\right| \leq 2$. The shaded beige region represents the overlapping of the $95 \%$ confidence interval for the consensus mean (green region) and the NIST range of tolerance (red region). 


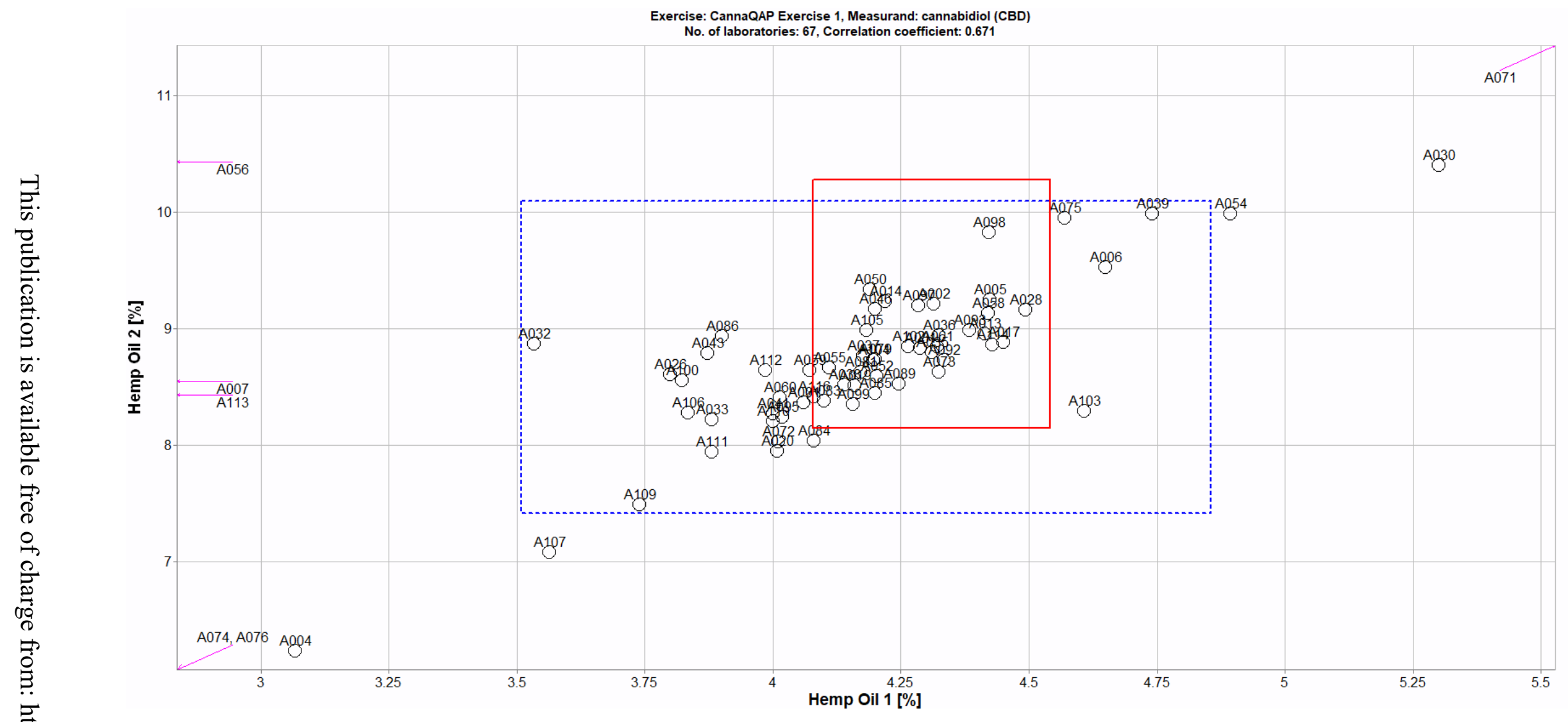

Figure 3-4. Laboratory means for CBD in Hemp Oil 1 and Hemp Oil 2 (sample/sample comparison view). In this view, the individual laboratory mean for one sample (Hemp Oil 1) is compared to the individual laboratory mean for a second sample (Hemp Oil 2). The solid red box represents the NIST range of tolerance for the two samples, Hemp Oil 1 (x-axis) and Hemp Oil 2 (y-axis), which encompasses the target values bounded by their uncertainties ( $\left.U_{\text {NIST }}\right)$ and represents the range that results in an acceptable $Z_{\text {NIST }}$ score, $\left|Z_{\text {NIST }}\right| \leq 2$. The dotted blue box represents the consensus range of tolerance for Hemp Oil 1 (x-axis) and Hemp Oil 2 (y-axis), calculated as the values above and below the consensus means that result in an acceptable $Z_{\text {comm }}^{\prime}$ score, $\left|Z_{\text {comm }}^{\prime}\right| \leq 2$. 
Table 3-3. Data summary table for CBDA in hemp oils. Data highlighted in red have been flagged as a data entry of zero or results that include text (e.g., "<LOQ" or "present"). Data highlighted in blue have been identified as outside the consensus tolerance limits and would be estimated to result in an unacceptable $Z_{\text {comm }}^{\prime}$ score, $\left|Z_{\text {comm }}^{\prime}\right| \geq 2$. Note: This table spans two pages; the NIST values and consensus values are included on both pages for convenience.

\begin{tabular}{|c|c|c|c|c|c|c|c|c|c|c|c|c|c|c|c|c|}
\hline & \multirow[b]{3}{*}{ Lab } & \multicolumn{15}{|c|}{ Cannabidiolic adid (CBDA) } \\
\hline & & \multicolumn{5}{|c|}{ Heap Oill (mass \%) } & \multicolumn{5}{|c|}{ He=p O11 2 (-mass \%) } & \multicolumn{5}{|c|}{ Henp O1 2a (-ass \%) } \\
\hline & & $\mathbf{A}$ & $\mathbf{B}$ & c & Avg & SD & $\mathbf{A}$ & $\mathbf{B}$ & $\mathbf{C}$ & Avg & SD & $\mathbf{A}$ & $\mathbf{B}$ & $\mathbf{C}$ & Avg & SD \\
\hline \multirow{49}{*}{ 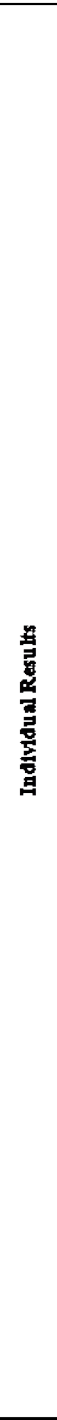 } & NIST & & & & & & & & & & & & & & & \\
\hline & $\mathrm{A} 001$ & $<0.15$ & $<0.15$ & $<0.15$ & $<0.15$ & & $<0.15$ & $<0.15$ & $<0.15$ & $<0.15$ & & & & & & \\
\hline & A002 & $<0.0043$ & $<0.0043$ & $<0.0043$ & $<0.0043$ & & $<0.0043$ & $<0.0043$ & $<0.0043$ & $<0.0043$ & & & & & & \\
\hline & $\mathrm{A} 003$ & & & & & & $<0.01$ & $<0.01$ & $<0.01$ & $<0.01$ & & & & & & \\
\hline & A004 & 0.02 & 0.02 & 0.02 & 0.0200 & 0.0000 & 0.02 & 0.02 & 0.02 & 0.0200 & 0.0000 & & & & & \\
\hline & $\mathrm{A} 005$ & 0.02 & 0.0191 & 0.021 & 0.0200 & 0.0010 & 0.015 & 0.0124 & 0.0141 & 0.0138 & 0.0013 & & & & & \\
\hline & A006 & 0.02 & & & 0.0200 & & 0.01 & & & 00100 & & & & & & \\
\hline & A 007 & & & & & & & & & & & & & & & \\
\hline & A008 & & & & & & & & & & & & & & & \\
\hline & A 009 & & & & & & & & & & & $<0.00001$ & $<0.00001$ & $<0.00001$ & $<0.00001$ & \\
\hline & A010 & & & & & & & & & & & & & & & \\
\hline & A 011 & & & & & & & & & & & & & & & \\
\hline & A012 & & & & & & 0 & 0 & 0 & 0.0000 & 0.0000 & & & & & \\
\hline & $\mathrm{A} 013$ & 0.014 & 0.015 & 0.015 & 0.0147 & 0.0006 & & & & & & & & & & \\
\hline & A014 & $<0.09$ & & & $<0.09$ & & & & & & & & & & & \\
\hline & A 015 & & & & & & & & & & & & & & & \\
\hline & A016 & & & & & & & & & & & & & & & \\
\hline & A 017 & 0.02 & & & 0.0200 & & 0.1 & & & 0.1000 & & & & & & \\
\hline & A018 & & & & & & & & & & & & & & & \\
\hline & A 019 & $<0.09$ & & & $<0.09$ & & $<0.09$ & & & $<0.09$ & & & & & & \\
\hline & A020 & 0.016051 & 0.015651 & 0.015359 & 0.0157 & 0.0003 & 0.005797 & 0.005608 & 000668 & 0.0060 & 0.0006 & & & & & \\
\hline & A 021 & & & & & & & & & & & $<0.05$ & $<0.05$ & $<0.05$ & $<0.05$ & \\
\hline & A022 & & & & & & & & & & & & & & & \\
\hline & A 023 & & & & & & 0 & 0 & 0 & 0.0000 & 0.0000 & & & & & \\
\hline & A024 & & & & & & & & & & & 0.015 & 0.016 & 0.016 & 0.0157 & 0.0006 \\
\hline & $\mathrm{A} 025$ & & & & & & & & & & & & & & & \\
\hline & A027 & & & & & & & & & & & $<0.0057$ & $<0.0057$ & $<0.0057$ & $<0.0057$ & \\
\hline & A 028 & $<0.0310$ & $<0.0310$ & $<0.0310$ & $<0.0310$ & & $<0.0310$ & $<0.0310$ & $<0.0310$ & $<0.0310$ & & & & & & \\
\hline & A030 & 0.02 & & & 00200 & & 0.016 & & & 0.0160 & & & & & & \\
\hline & A 031 & $<0.15$ & $<0.15$ & $<0.15$ & $<0.15$ & & $<0.15$ & $<0.15$ & $<0.15$ & $<0.15$ & & & & & & \\
\hline & A033 & 0.03 & 0.03 & 0.02 & 0.0267 & 0.0058 & 0 & 0 & 0 & 0.0000 & 0.0000 & & & & & \\
\hline & A 034 & & & & & & $<0.01$ & $<0.01$ & $<0.01$ & $<0.01$ & & & & & & \\
\hline & A035 & 0.0202 & & & 0.0202 & & $<0.0025$ & $<0.0025$ & $<0.0025$ & $<0.0025$ & & & & & & \\
\hline & A 036 & $<0.247$ & $<0.247$ & $<0.247$ & $<0.247$ & & $<0.247$ & $<0.247$ & $<0.247$ & $<0.247$ & & & & & & \\
\hline & A037 & $<0.05$ & $<0.05$ & $<0.05$ & $<0.05$ & & $<0.05$ & $<0.05$ & $<0.05$ & $<0.05$ & & & & & & \\
\hline & A 038 & 0.0119 & 0.0117 & 0.012 & 0.0119 & 0.0002 & $<0.025$ & $<0.025$ & $<0.025$ & $<0.025$ & & & & & & \\
\hline & A039 & 0.04 & 0.05 & 0.04 & 0.0433 & 0.0058 & 0.03 & 0.02 & 0.03 & 0.0267 & 0.0058 & & & & & \\
\hline & A 040 & & & & & & & & & & & ND & ND & ND & & \\
\hline & A041 & 0 & 0 & 0 & 0.0000 & 0.0000 & 0 & 0 & 0 & 0.0000 & 0.0000 & & & & & \\
\hline & A 043 & 0.024 & 0.024 & 0.025 & 0.0243 & 0.0006 & 0.014 & 0.014 & 0.015 & 0.0143 & 0.0006 & & & & & \\
\hline & A044 & & & & & & & & & & & & & & & \\
\hline & A 045 & & & & & & & & & & & & & & & \\
\hline & A046 & 0 & 0 & 0 & 0.0000 & 0.0000 & 0 & 0 & 0 & 0.0000 & 0.0000 & & & & & \\
\hline & $\mathrm{A} 050$ & 0.0201 & 0.019 & 0.0221 & 0.0204 & 0.0016 & $<0.01$ & $<0.01$ & $<0.01$ & $<0.01$ & & & & & & \\
\hline & A051 & & & & & & & & & & & & & & & \\
\hline & A 052 & & & & & & & & & & & & & & & \\
\hline & A053 & & & & & & & & & & & & & & & \\
\hline & A 054 & $<0.01$ & $<0.01$ & $<0.01$ & $<0.01$ & & $<0.01$ & $<0.01$ & $<0.01$ & $<0.01$ & & & & & & \\
\hline & A055 & 0.0213 & 0.0223 & 0.0213 & 0.0216 & 0.0006 & 0 & 0 & 0 & 0.0000 & 0.0000 & & & & & \\
\hline 覃 & & \begin{tabular}{|l} 
Consensus \\
Consensus \\
Maximum \\
Minimum \\
N \\
\end{tabular} & $\begin{array}{l}\text { s Mean } \\
\text { s Standard I } \\
\end{array}$ & Deviation & $\begin{array}{c}0.0183 \\
0.0017 \\
0.1633 \\
0.0000 \\
31\end{array}$ & & \begin{tabular}{|l|} 
Consensu \\
Consensu \\
Maximum \\
Minimum \\
N \\
\end{tabular} & $\begin{array}{l}\text { is Mean } \\
\text { Is Standard I } \\
\text { a }\end{array}$ & Deviation & $\begin{array}{c}0.0098 \\
0.0026 \\
0.3900 \\
0.0000 \\
28\end{array}$ & & \begin{tabular}{|l} 
Consensus \\
Consensus \\
Maximum \\
Minimum \\
$\mathrm{N}$
\end{tabular} & $\begin{array}{l}\text { S Mean } \\
\text { S Standand I } \\
\end{array}$ & Deviation & $\begin{array}{c}0.0080 \\
0.0063 \\
0.0157 \\
0.0000 \\
3\end{array}$ & \\
\hline
\end{tabular}




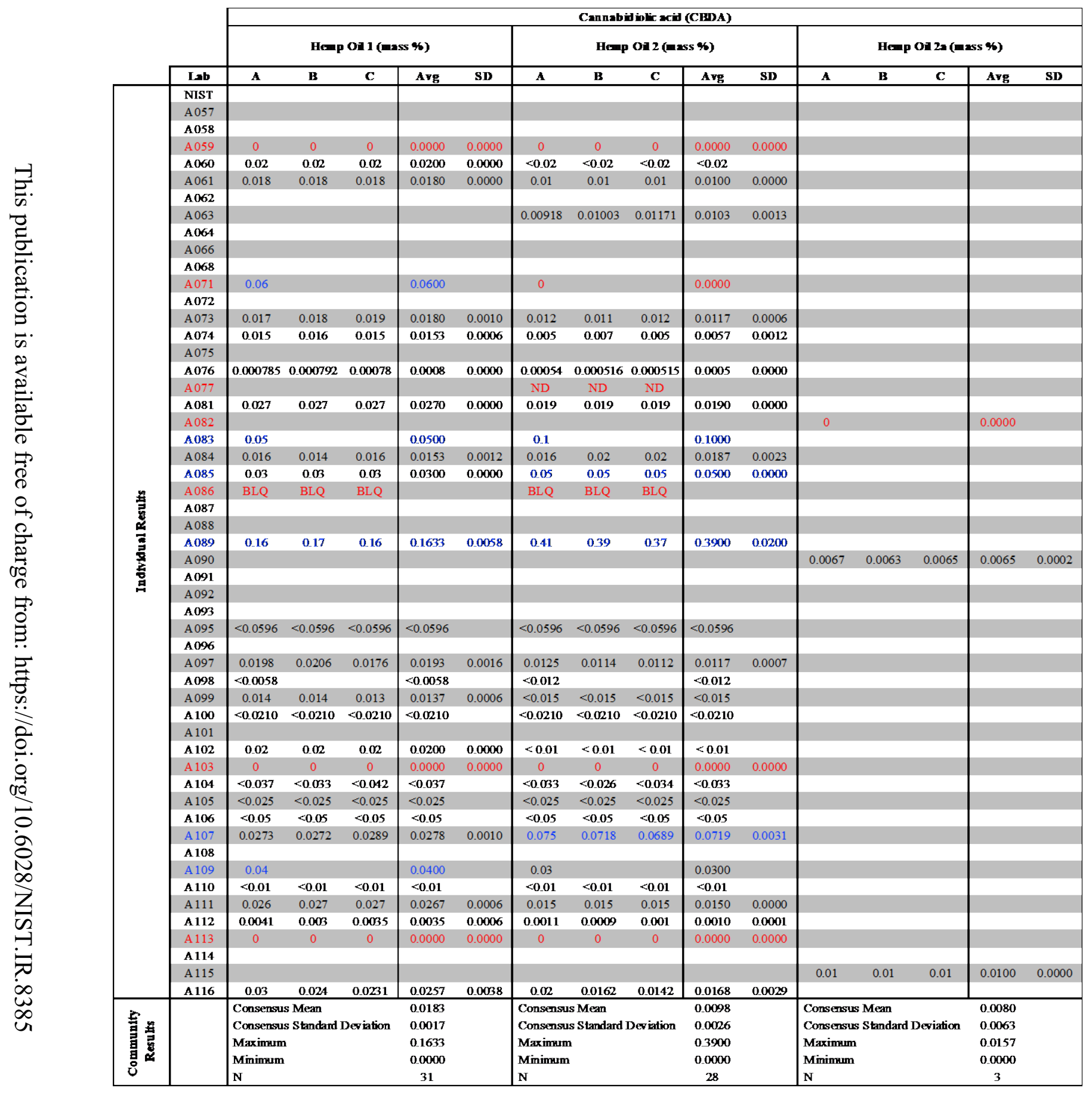




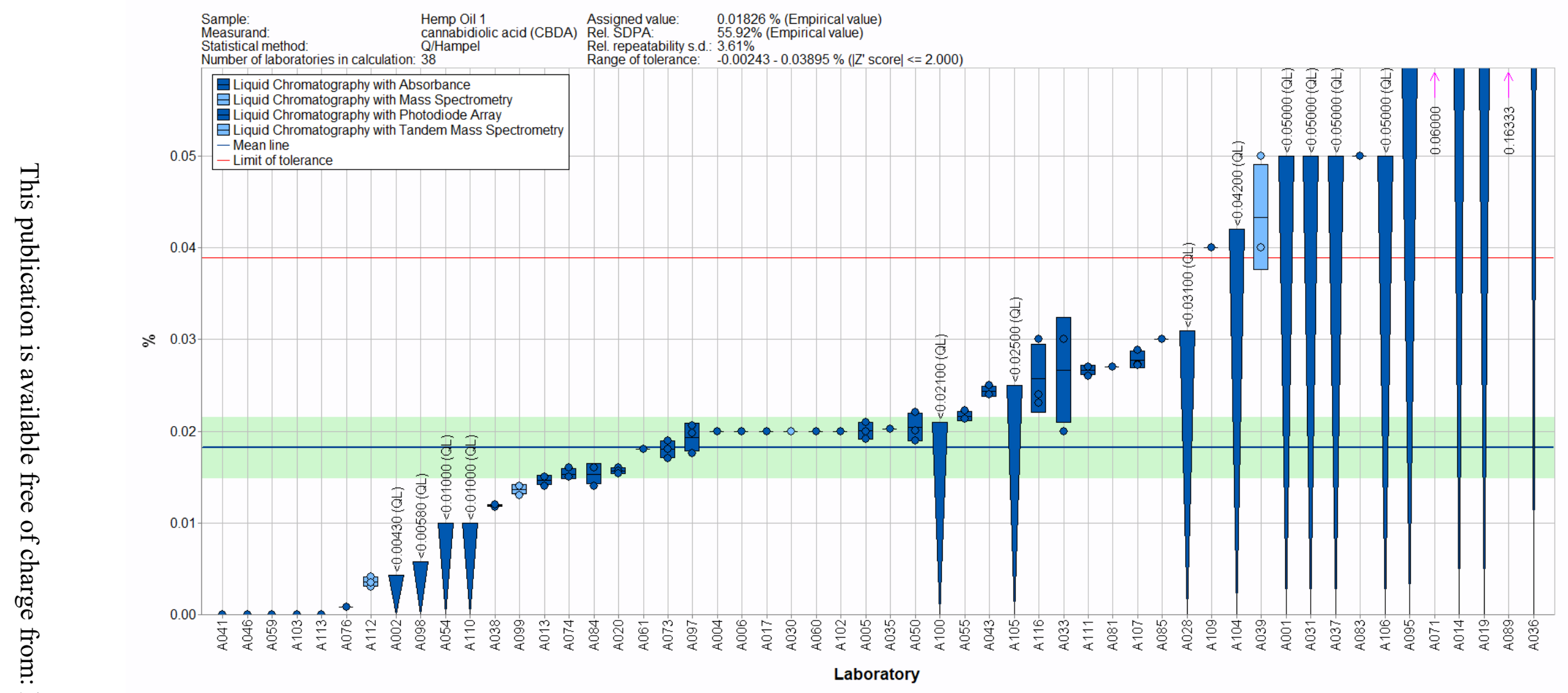

Figure 3-5. CBDA in Hemp Oil 1 (data summary view - analytical method). In this view, individual laboratory data are plotted (circles) with the individual laboratory standard deviation (rectangle). The color of the data point represents the analytical method employed. The solid blue line represents the consensus mean, and the green shaded region represents the $95 \%$ confidence interval for the consensus mean. The solid red line represents the upper consensus range of tolerance, calculated as the values above the consensus mean that results in an acceptable $Z_{\text {comm }}^{\prime}$ score, $\left|Z_{\text {comm }}^{\prime}\right| \leq 2$, with the lower limit set at zero. A NIST value has not been determined in this material. The downward triangle represents data reported as a threshold or LOQ value. 


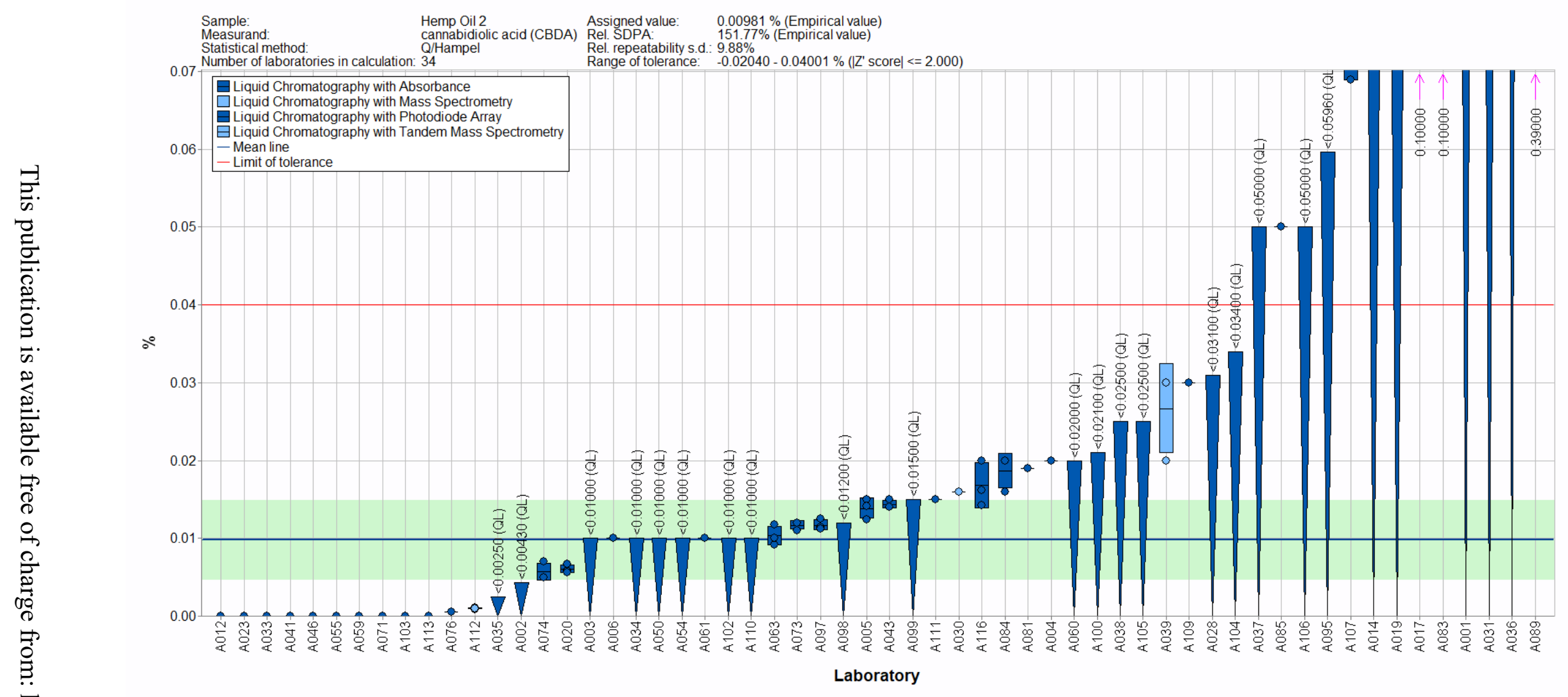

Figure 3-6. CBDA in Hemp Oil 2 (data summary view - analytical method). In this view, individual laboratory data are plotted (circles) with the individual laboratory standard deviation (rectangle). The color of the data point represents the analytical method employed. The solid blue line represents the consensus mean, and the green shaded region represents the $95 \%$ confidence interval for the consensus mean. The solid red line represents the upper consensus range of tolerance, calculated as the values above the consensus mean that results in an acceptable $Z_{\text {comm }}^{\prime}$ score, $\left|Z_{\text {comm }}^{\prime}\right| \leq 2$, with the lower limit set at zero. A NIST value has not been determined in this material. The downward triangle represents data reported as a threshold or LOQ value. 


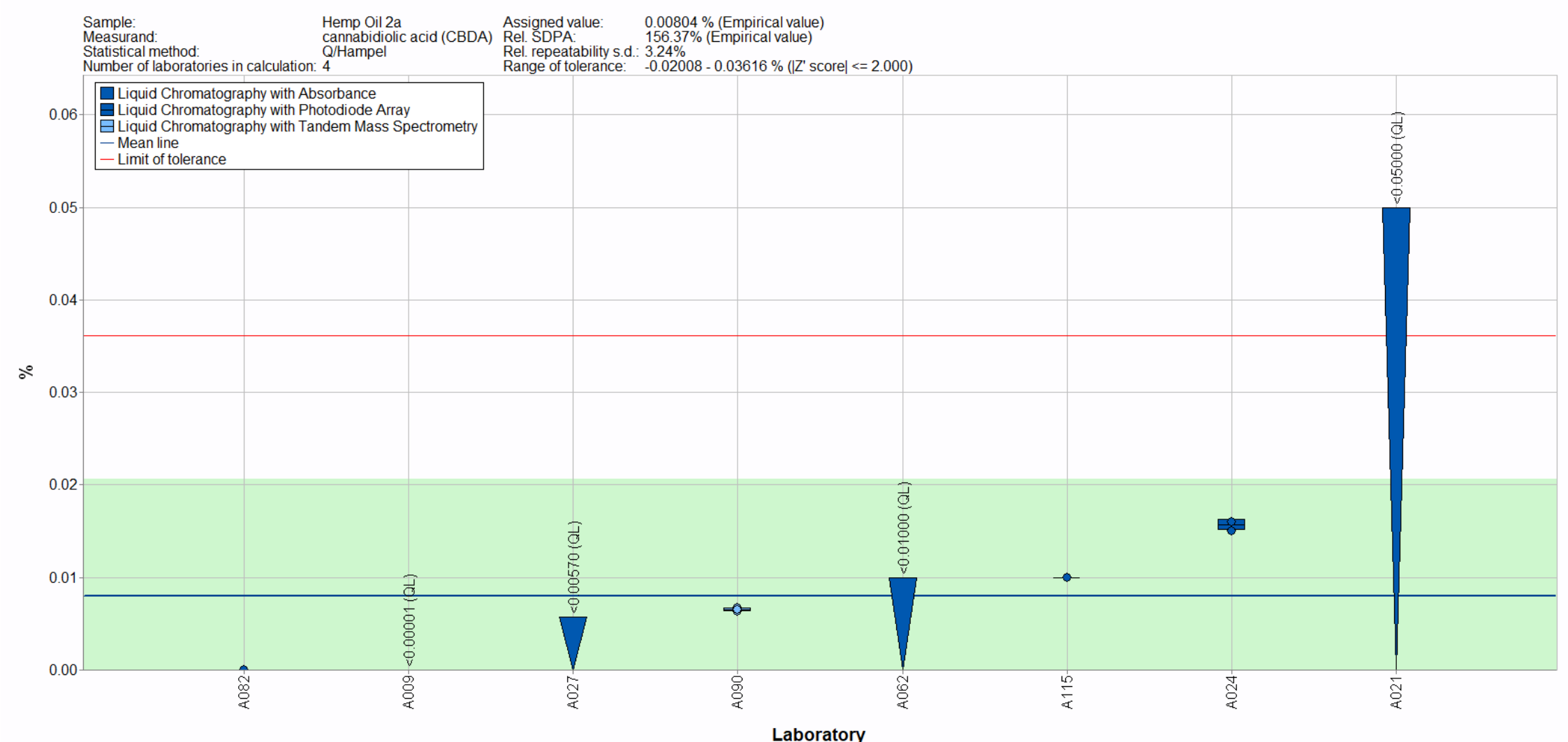

Figure 3-7. CBDA in Hemp Oil 2a (data summary view - analytical method). In this view, individual laboratory data are plotted (circles) with the individual laboratory standard deviation (rectangle). The color of the data point represents the analytical method employed. The solid blue line represents the consensus mean, and the green shaded region represents the $95 \%$ confidence interval for the consensus mean. The solid red line represents the upper consensus range of tolerance, calculated as the values above the consensus mean that results in an acceptable $Z_{\text {comm }}^{\prime}$ score, $\left|Z_{\text {comm }}^{\prime}\right| \leq 2$, with the lower limit set at zero. A NIST value has not been determined in this material. The downward triangle represents data reported as a threshold or LOQ value. 


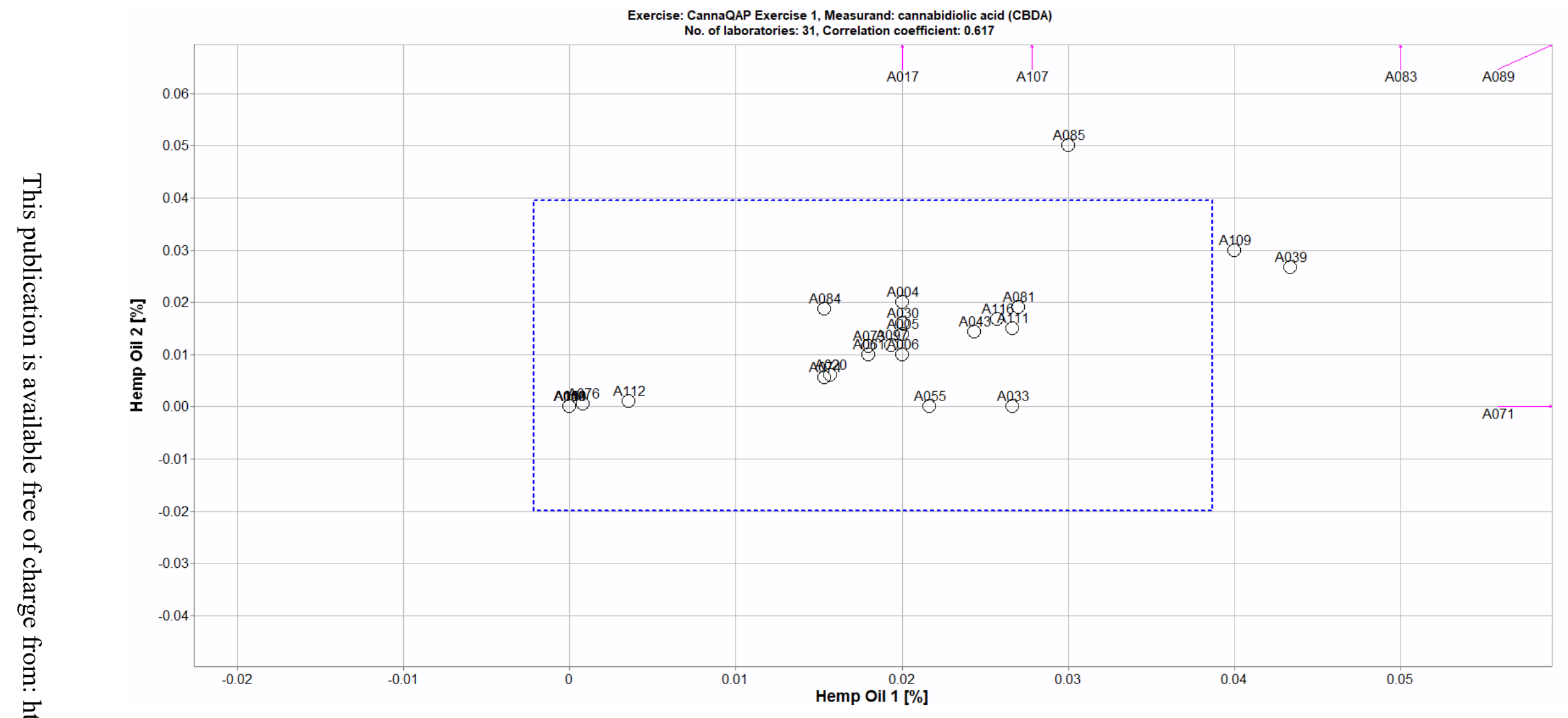

Figure 3-8. Laboratory means for CBDA in Hemp Oil 1 and Hemp Oil 2 (sample/sample comparison view). In this view, the individual laboratory mean for one sample (Hemp Oil 1) is compared to the individual laboratory mean for a second sample (Hemp Oil 2). The dotted blue box represents the consensus range of tolerance for Hemp Oil 1 (x-axis) and Hemp Oil 2 (y-axis), calculated as the values above and below the consensus means that result in an acceptable $Z_{\text {comm }}^{\prime}$ score, $\left|Z_{\text {comm }}^{\prime}\right| \leq 2$. 
Table 3-4. Data summary table for total CBD in hemp oils. Data highlighted in blue have been identified as outside the consensus tolerance limits and would be estimated to result in an unacceptable $Z_{\text {comm }}^{\prime}$ score, $\left|Z_{\text {comm }}^{\prime}\right| \geq 2$. Note: This table spans two pages; the NIST values and consensus values are included on both pages for convenience.

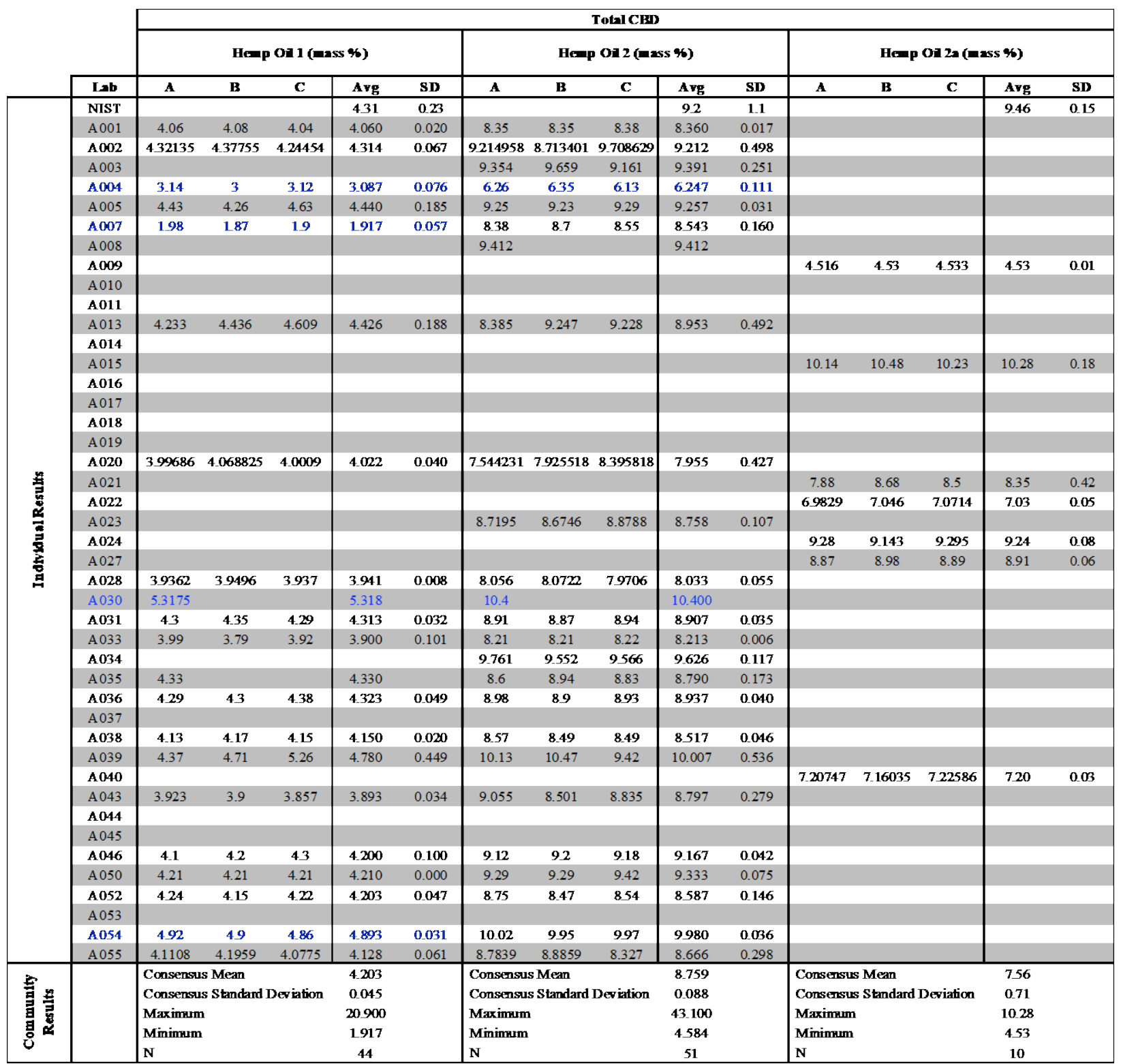




\begin{tabular}{|c|c|c|c|c|c|c|c|c|c|c|c|c|c|c|c|c|c|}
\hline & & & & & & & & & & [otalCBD & & & & & & & \\
\hline & & & & He= & Oil 1 (m) & 5 \%) & & & He=p & ON 2 (ma & s \%) & & & $\mathrm{He}$ & OAl 20 & $5 \%)$ & \\
\hline & & Lab & $\mathbf{A}$ & B & $\mathbf{C}$ & Avg & SD & $\mathbf{A}$ & B & $\mathbf{C}$ & Avg & SD & $\mathbf{A}$ & B & $\mathbf{C}$ & Avg & SD \\
\hline & & $\begin{array}{l}\text { NIST } \\
\text { A056 }\end{array}$ & & & & 431 & 023 & & & & 92 & 1.1 & & & & 9.46 & 0.15 \\
\hline & & A 057 & & & & & & & & & & & & & & & \\
\hline & & A058 & 4365 & 4.468 & 4.457 & 4.430 & 0.057 & 9.03 & 9.076 & 9.278 & 9.128 & 0.132 & & & & & \\
\hline & & A 059 & 4.0275 & 4.124 & 4.062 & 4.071 & 0.049 & 8.6445 & 8.629 & 8.647 & 8.640 & 0.010 & & & & & \\
\hline$\square$ & & $A 060$ & 4.01 & 4.03 & 4.05 & 4.030 & 0.020 & 839 & 836 & 8.47 & 8.407 & 0.057 & & & & & \\
\hline$\vec{\sim}$. & & A 061 & 4.335 & 4.363 & 4.312 & 4.337 & 0.026 & 8.932 & 8.746 & 8.886 & 8.855 & 0.097 & & & & & \\
\hline$\sigma$ & & A062 & & & & & & & & & & & 5594 & 5588 & 5.749 & 5.64 & 0.09 \\
\hline 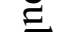 & & A 063 & & & & & & 7.60595 & 7.70831 & 7.68022 & 7.665 & 0.053 & & & & & \\
\hline$\underline{\sigma}$ & & A064 & & & & & & & & & & & & & & & \\
\hline$\vec{\rho}$. & & A 065 & 4.635 & 4.54 & 4.495 & 4.557 & 0.071 & 8.78 & 9.965 & 10.05 & 9.598 & 0.710 & & & & & \\
\hline$\stackrel{2}{ح}$ & & A066 & & & & & & & & & & & & & & & \\
\hline 0 & & A 068 & & & & & & & & & & & & & & & \\
\hline 追 & & $A 071$ & 209 & & & 20900 & & 43.1 & & & 43.100 & & & & & & \\
\hline$\vec{\sim}$. & & A 072 & 4.01 & & & 4.010 & & 8.03 & & & 8.030 & & & & & & \\
\hline ט & & A073 & 4305 & 4376 & 4.337 & 4339 & 0.036 & 8.646 & 8.62 & 8.646 & 8.637 & 0.015 & & & & & \\
\hline$\stackrel{2}{2}$ & & A 074 & 2.901 & 2.362 & 3.045 & 2.769 & 0.360 & 4.408 & 4.789 & 4.555 & 4.584 & 0.192 & & & & & \\
\hline ڤ. & & A075 & & & & & & & & & & & & & & & \\
\hline$\tilde{D}$ & & A 076 & & & & & & & & & & & & & & & \\
\hline$\tilde{\sigma}$ & & A077 & & & & & & 8.77 & 8.85 & 93 & 8973 & 0286 & & & & & \\
\hline$\overline{0}$ & & A 078 & & & & & & & & & & & & & & & \\
\hline 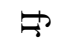 & & A081 & 4.186 & 4.183 & 4.194 & 4.188 & 0.006 & 8.619 & 8.652 & 8665 & 8.645 & 0.024 & & & & & \\
\hline 8 & & A 082 & & & & & & & & & & & & & & & \\
\hline 0 & 急 & A083 & & & & & & & & & & & & & & & \\
\hline$\stackrel{0}{\longrightarrow}$ & 局 & A 084 & & & & & & & & & & & & & & & \\
\hline$\rho$ & 뜰 & A085 & 4.23 & 4. 16 & 43 & 4.230 & 0.070 & 8.48 & 8.52 & 8.45 & $\mathbf{8 . 4 8 3}$ & 0.035 & & & & & \\
\hline$\ddot{0}$ & 3 & A 086 & 3.5 & 4 & 4.2 & 3.900 & 0.361 & 8.9 & 8.9 & 9 & 8.933 & 0.058 & & & & & \\
\hline$\stackrel{0}{=}$ & $\frac{5}{8}$ & A 087 & & & & & & & & & & & & & & & \\
\hline 0 & & A 088 & & & & & & 8.87 & 9.06 & 9.15 & 9.027 & 0.143 & & & & & \\
\hline$\rightarrow$ & & A089 & 427 & 4.34 & 433 & 4313 & 0.038 & 9.24 & 9.42 & 794 & 8.867 & 0.808 & & & & & \\
\hline $\overrightarrow{0}^{\prime}$ & & A 090 & & & & & & & & & & & 6.5749 & 6.45 & 6.4781 & 6.50 & 0.07 \\
\hline 当 & & A091 & & & & & & & & & & & & & & & \\
\hline- & & A 092 & 4.2743 & 4.3643 & 4.361 & 4.333 & 0.051 & 8.7712 & 8.6602 & 8.7468 & 8.726 & 0.058 & & & & & \\
\hline$\Xi$ & & $\mathrm{A} 093$ & 4.12 & 4.7 & 433 & 4383 & 0.294 & 9.07 & 8.85 & 9.02 & 8980 & 0.115 & & & & & \\
\hline 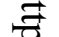 & & A 095 & 4.0425 & 3.9904 & 4.0222 & 4.018 & 0.026 & 8.3004 & 8.1306 & 8.2899 & 8.240 & 0.095 & & & & & \\
\hline ర & & A096 & & & & & & & & & & & & & & & \\
\hline$\ddot{亠}$ & & A 097 & 4.5068 & 4.0208 & 4.3776 & 4.302 & 0.252 & 8.7654 & 9.3035 & 9.553 & 9.207 & 0.403 & & & & & \\
\hline 를 & & A099 & 4.139 & 4.168 & 4.198 & 4.168 & 0.030 & $\mathbf{8 . 4 0 7}$ & 8.169 & $\mathbf{8 . 4 7 2}$ & 8349 & 0.160 & & & & & \\
\hline O & & A 100 & 3.686 & 3.859 & 3.921 & 3.822 & 0.122 & 8.723 & 8.423 & 8.502 & 8.549 & 0.156 & & & & & \\
\hline$\dot{c}$ & & A 101 & & & & & & & & & & & & & & & \\
\hline ब0 & & A 102 & 4.31 & 4.26 & 4.28 & 4.283 & 0.025 & 8.8 & 8.88 & 8.86 & 8.847 & 0.042 & & & & & \\
\hline 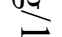 & & A 103 & 4.608 & 4.719 & 4.496 & 4.608 & 0.112 & 7.242 & 8.757 & 8.874 & 8.291 & 0910 & & & & & \\
\hline- & & A 104 & 4.134 & 4.221 & 4.234 & 4.196 & 0.054 & 8.803 & 8.73 & 8.648 & 8.727 & 0.078 & & & & & \\
\hline a & & A 105 & 4.29 & 4.13 & 4.13 & 4.183 & 0.092 & 9 & 8.95 & 9.01 & 8987 & 0.032 & & & & & \\
\hline 0 & & A 107 & 3.71 & 3.43 & 3.62 & 3.587 & 0.143 & 7.56 & 6.7 & 7.17 & 7.143 & 0.431 & & & & & \\
\hline$\infty$ & & A 108 & & & & & & & & & & & & & & & \\
\hline$\infty$ & & A 109 & 3.77 & 3.71 & & 3.740 & 0.042 & 7.46 & 7.51 & & 7.485 & 0.035 & & & & & \\
\hline 7 & & A110 & & & & & & & & & & & & & & & \\
\hline$\vec{\Omega}$ & & A 111 & 3.69 & 4.18 & 3.86 & 3.910 & 0.249 & 7.8 & 8.48 & 7.59 & 7.957 & 0.465 & & & & & \\
\hline & & A112 & & & & & & & & & & & & & & & \\
\hline$\dot{z}$ & & A 114 & 4.4251 & 4.4441 & 4.4174 & 4.429 & 0.014 & 8.7951 & 8.8643 & 8.9147 & 8.858 & 0.060 & & & & & \\
\hline ? & & A115 & & & & & & & & & & & 8 & 7.81 & 8.03 & 795 & 0.12 \\
\hline $\begin{array}{l}\infty \\
\infty \\
\infty\end{array}$ & 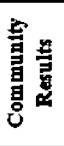 & & \begin{tabular}{|l} 
Consensu \\
Consensu \\
Maximum \\
Minimum \\
$\mathrm{N}$
\end{tabular} & $\begin{array}{l}\text { Mean } \\
\text { Standard }\end{array}$ & eviation & $\begin{array}{c}4.203 \\
0.045 \\
20.900 \\
1917 \\
44\end{array}$ & & $\begin{array}{l}\text { Consensus } \\
\text { Consensus } \\
\text { Maximum } \\
\text { Minimum } \\
\mathrm{N}\end{array}$ & $\begin{array}{l}\text { Mean } \\
\text { Stendard }\end{array}$ & Deviation & $\begin{array}{c}8.759 \\
0.088 \\
43.100 \\
4.584 \\
51\end{array}$ & & $\begin{array}{l}\text { Consensu } \\
\text { Consensu } \\
\text { Maximum } \\
\text { Minimum } \\
\text { N }\end{array}$ & $\begin{array}{l}\text { Mean } \\
\text { Standand }\end{array}$ & eviation & $\begin{array}{c}7.56 \\
0.71 \\
10.28 \\
453 \\
10\end{array}$ & \\
\hline
\end{tabular}




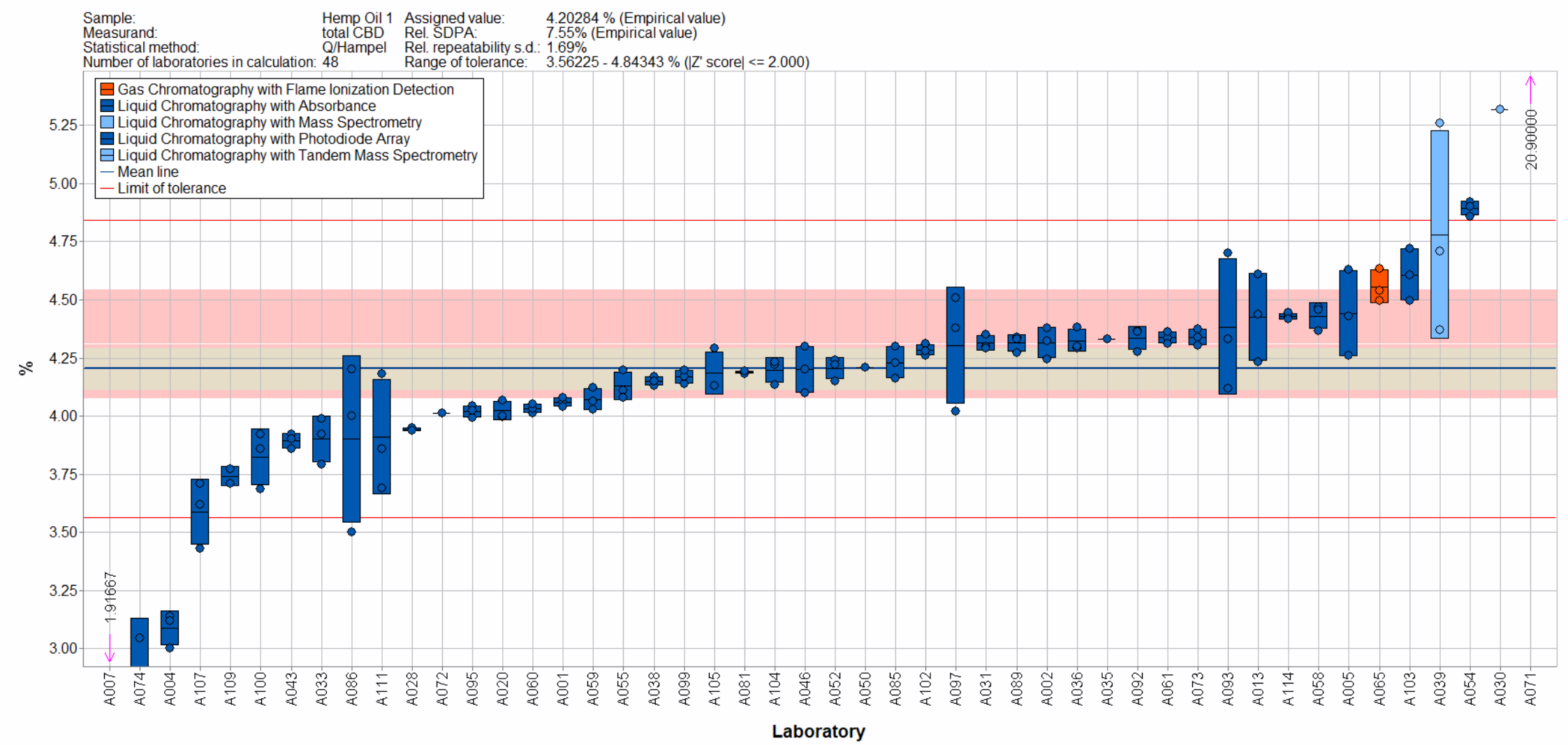

Figure 3-9. Total CBD in Hemp Oil 1 (data summary view - analytical method). In this view, individual laboratory data are plotted (circles) with the individual laboratory standard deviation (rectangle). The color of the data point represents the analytical method employed. The solid blue line represents the consensus mean, and the green shaded region represents the $95 \%$ confidence interval for the consensus mean. The solid red lines represent the consensus range of tolerance, calculated as the values above and below the consensus mean that result in an acceptable $Z_{\text {comm }}^{\prime}$ score, $\left|Z_{\text {comm }}^{\prime}\right| \leq 2$. The red shaded region represents the NIST range of tolerance, which encompasses the target value bounded by twice its uncertainty ( $\left.U_{\mathrm{NIST}}\right)$ and represents the range that results in an acceptable $Z_{\mathrm{NIST}}$ score, $\left|Z_{\mathrm{NIST}}\right| \leq 2$. The shaded beige region represents the overlapping of the $95 \%$ confidence interval for the consensus mean (green region) and the NIST range of tolerance (red region). 


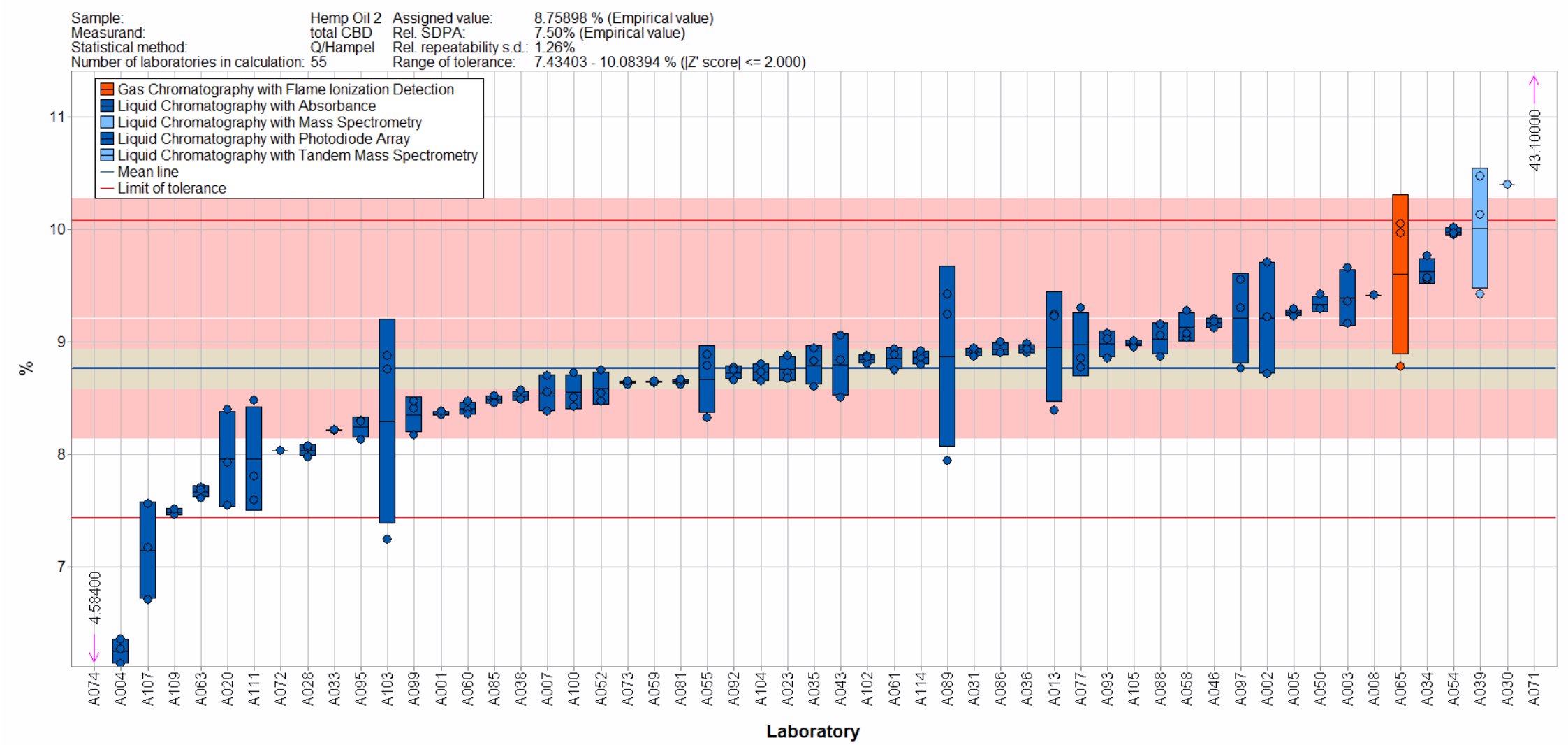

Figure 3-10. Total CBD in Hemp Oil 2 (data summary view - analytical method). In this view, individual laboratory data are plotted (circles) with the individual laboratory standard deviation (rectangle). The color of the data point represents the analytical method employed. The solid blue line represents the consensus mean, and the green shaded region represents the $95 \%$ confidence interval for the consensus mean. The solid red lines represent the consensus range of tolerance, calculated as the values above and below the consensus mean that result in an acceptable $Z_{\text {comm }}^{\prime}$ score, $\left|Z_{\text {comm }}^{\prime}\right| \leq 2$. The red shaded region represents the NIST range of tolerance, which encompasses the target value bounded by twice its uncertainty ( $\left.U_{\mathrm{NIST}}\right)$ and represents the range that results in an acceptable $Z_{\mathrm{NIST}}$ score, $\left|Z_{\mathrm{NIST}}\right| \leq 2$. The shaded beige region represents the overlapping of the $95 \%$ confidence interval for the consensus mean (green region) and the NIST range of tolerance (red region). 


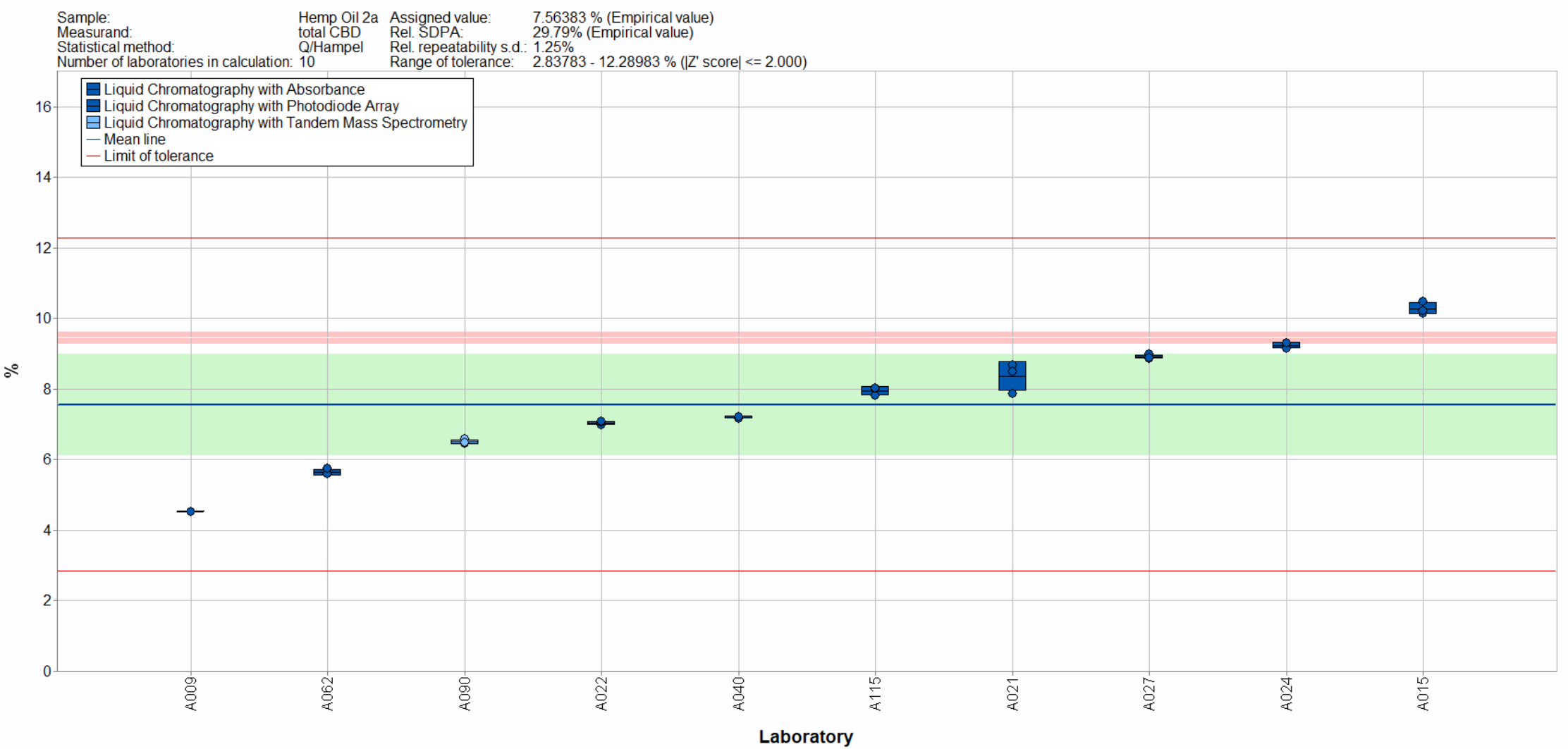

Figure 3-11. Total CBD in Hemp Oil 2a (data summary view - analytical method). In this view, individual laboratory data are plotted (circles) with the individual laboratory standard deviation (rectangle). The color of the data point represents the analytical method employed. The solid blue line represents the consensus mean, and the green shaded region represents the $95 \%$ confidence interval for the consensus mean. The solid red lines represent the consensus range of tolerance, calculated as the values above and below the consensus mean that result in an acceptable $Z_{\text {comm }}^{\prime}$ score, $\left|Z_{\text {comm }}^{\prime}\right| \leq 2$. The red shaded region represents the NIST range of tolerance, which encompasses the target value bounded by twice its uncertainty ( $\left.U_{\mathrm{NIST}}\right)$ and represents the range that results in an acceptable $Z_{\mathrm{NIST}}$ score, $\left|Z_{\mathrm{NIST}}\right| \leq 2$. The shaded beige region represents the overlapping of the $95 \%$ confidence interval for the consensus mean (green region) and the NIST range of tolerance (red region). 


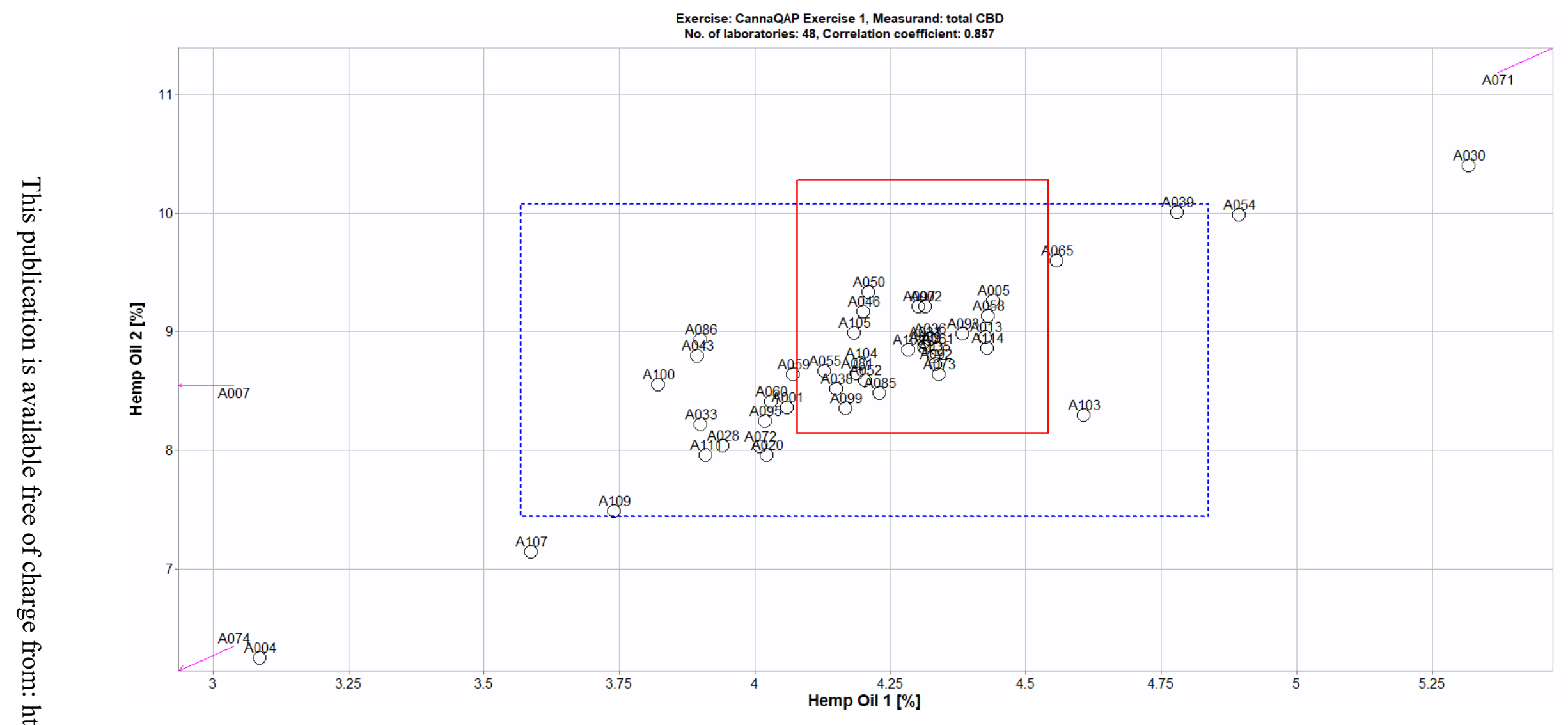

Figure 3-12. Laboratory means for total CBD in Hemp Oil 1 and Hemp Oil 2 (sample/sample comparison view). In this view, the individual laboratory mean for one sample (Hemp Oil 1) is compared to the individual laboratory mean for a second sample (Hemp Oil 2). The solid red box represents the NIST range of tolerance for the two samples, Hemp Oil 1 (x-axis) and Hemp Oil 2 (y-axis), which encompasses the target values bounded by their uncertainties ( $\left.U_{N I S T}\right)$ and represents the range that results in an acceptable $Z_{\mathrm{NIST}}$ score, $\left|Z_{\text {NIST }}\right| \leq 2$. The dotted blue box represents the consensus range of tolerance for Hemp Oil 1 (x-axis) and Hemp Oil 2 (y-axis), calculated as the values above and below the consensus means that result in an acceptable $Z_{\text {comm }}^{\prime}$ score, $\left|Z_{\text {comm }}^{\prime}\right| \leq 2$. 


\section{SECTION 4: CBC AND CBCA}

Study Overview

$\mathrm{CBC}$ is a non-intoxicating cannabinoid that has attracted significant interest due to research showing potential health benefits for humans. ${ }^{8} \mathrm{CBC}$ is one of the most commonly identified cannabinoids in Cannabis plants and Cannabis-derived products, and reliable analytical methods are needed to better understand the health impacts of $\mathrm{CBC}$ consumption. $\mathrm{CBC}$ does not exist in Cannabis plant naturally but is formed following decarboxylation of its acidic precursor (CBCA) by exposure to heat or light. Participants in this study were asked to use in-house analytical methods to determine the mass fraction (\%) of CBC and CBCA in three hemp oil samples. The preparation of these hemp oils included a decarboxylation step resulting in extremely low levels of $\mathrm{CBCA}$ and levels of $\mathrm{CBC}$ in normal commercial product ranges.

\section{$\underline{\text { Reporting Statistics }}$}

- The enrollment and reporting statistics for CBC and CBCA are described in the table below for each analyte. Reported values may include non-quantitative results (zero or below LOQ).

\begin{tabular}{cccccccc} 
& \multicolumn{2}{c}{ Hemp Oil 1 } & \multicolumn{2}{c}{ Hemp Oil 2 } & & \multicolumn{2}{c}{ Hemp Oil 2a } \\
& $\underline{\text { Number of }}$ & $\underline{\underline{\text { Reporting }}}$ & & $\underline{\text { Number of }}$ & $\underline{\underline{\text { Reporting }}}$ & $\underline{\text { Number of }}$ & $\underline{\underline{\text { Reporting }}}$ \\
$\underline{\text { Analyte }}$ & $\underline{\text { Participants }}$ & $\underline{\text { Results }}$ & $\underline{\text { Participants }}$ & $\underline{\text { Results }}$ & $\underline{\text { Participants }}$ & $\underline{\text { Results }}$ \\
CBC & 63 & $84 \%$ & 71 & $85 \%$ & 19 & $63 \%$ \\
CBCA & 36 & $56 \%$ & 38 & $55 \%$ & 19 & $21 \%$
\end{tabular}

- Most laboratories reported using solvent extraction or sample dilution for determination of CBC and CBCA in the three hemp oil samples (see table below). Additional sample preparation details are summarized at the end of the report in Appendix I.

\begin{tabular}{ccc} 
Reported & \multicolumn{2}{c}{ Percent Reporting } \\
$\underline{\text { Preparation Method }}$ & $\underline{\text { CBC }}$ & $\underline{\text { CBCA }}$ \\
Solvent Extraction & 71.1 & 68.3 \\
Dilution & 21.9 & 26.7 \\
Other & 0.0 & 0.0 \\
None & 2.3 & 1.7 \\
No Response & 4.7 & 3.3
\end{tabular}

${ }^{8}$ M Zagozen, A Cerenak, S Kreft. Acta Pharm. 71: 355-364 (2021) https://doi.org/10.2478/acph-2021-0021. 
- Most laboratories reported using LC-PDA or LC-UV for the determination of CBC and CBCA in the three hemp oil samples (see table below). Additional method details are summarized at the end of the report in Appendix I.

$\begin{array}{ccc}\begin{array}{c}\text { Reported } \\ \text { Analytical Method }\end{array} & \frac{\text { CBC }}{\text { Percent Reporting }} \\ \text { LC-PDA } & 68.3 & \frac{\text { CBCA }}{57.7} \\ \text { LC-UV } & 28.1 & 36.7 \\ \text { LC-MS } & 1.6 & 3.3 \\ \text { LC-MS/MS } & 4.7 & 0.0 \\ \text { GC-FID } & 0.0 & 0.0 \\ \text { GC-MS } & 2.3 & 0.0 \\ \text { Other } & 0.0 & 3.3\end{array}$

$\underline{\text { Study Results }}$

$C B C$

- The mass fractions (\%) for CBC in the hemp oil samples were determined by NIST using LC-PDA as described in Section 1 and are summarized in Table 4-1. These NIST values are used as the target means and ranges summarized in Table 4-2 for comparison to the participant results.

- The target and consensus means and ranges are summarized for $\mathrm{CBC}$ via different analytical methods in Figure 4-1, Figure 4-2, and Figure 4-3, which include data from laboratories submitting two or three results for CBC. Data from participants submitting only one measurement were included in Table 4-2 but were not included in the calculation of consensus statistics. ${ }^{2}$

- For CBC in Hemp Oil 1, the consensus range was based on quantitative results from 53 laboratories and does not overlap with the target range (Figure 4-1).

- The individual laboratory means or thresholds from 49 laboratories $(93 \%$ of those reporting results) were outside the NIST range of tolerance for CBC in Hemp Oil 1.

- The individual laboratory means from 9 laboratories (17\% of those reporting results) were outside the acceptable $Z_{\text {comm }}^{\prime}$ score for CBC in Hemp Oil 1.

- No results were reported using thresholds or LOQs for CBC in Hemp Oil 1.

- For CBC in Hemp Oil 2, the consensus range was based on quantitative results from 59 laboratories and overlaps $20 \%$ of the target range (Figure 4-2).

- The individual laboratory means or thresholds from 42 laboratories $(71 \%$ of those reporting results) were outside the NIST range of tolerance for CBC in Hemp Oil 2.

- The individual laboratory means from 9 laboratories (15\% of those reporting results) were outside the acceptable $Z_{\text {comm }}^{\prime}$ score for CBC in Hemp Oil 2.

- No results were reported using thresholds or LOQs for CBC in Hemp Oil 2.

- For CBC in Hemp Oil 2a, the consensus range was based on quantitative results from 11 laboratories and complete overlaps within the target range (Figure 4-3).

- The individual laboratory means or thresholds from 2 laboratories $(18 \%$ of those reporting results) were outside the NIST range of tolerance for CBC in Hemp Oil 2a. 
- The individual laboratory mean from 1 laboratory ( $9 \%$ of those reporting results) was outside the acceptable $Z_{\text {comm }}^{\prime}$ score for CBC in Hemp Oil 2a.

- No results were reported using thresholds or LOQs for CBC in Hemp Oil 2a.

- A comparison of individual laboratory means for CBC in Hemp Oil 1 and Hemp Oil 2 is summarized in Figure 4-4 for laboratories who reported results for both samples.

$C B C A$

- No target means or ranges were provided in Table 4-1 for CBCA in the three hemp oils.

- The consensus means and ranges for CBCA are based on quantitative data from 4 laboratories (Figure 4-5), 5 laboratories (Figure 4-6) for Hemp Oil 1 and Hemp Oil 2, respectively. A consensus mean could not be determined for CBCA in Hemp Oil 2a (Figure 4-7). Data from participants submitting only one measurement were included in Table 4-3 but were not included in the calculation of consensus statistics. ${ }^{2}$

- A comparison of individual laboratory means for CBCA in Hemp Oil 1 and Hemp Oil 2 is summarized in Figure 4-8 for laboratories who reported results for both samples.

Overall

- The between-laboratory variabilities for determination of $\mathrm{CBC}$ and $\mathrm{CBCA}$ in the hemp oil samples are shown in the table below.

Between-Laboratory Variability (\% RSD)

\begin{tabular}{|c|c|c|c|}
\hline Analyte & Hemp Oil 1 & Hemp Oil 2 & Hemp Oil 2a \\
\hline $\mathrm{CBC}$ & 1.6 & 1.4 & 6.0 \\
\hline $\mathrm{CBCA}$ & 136.9 & 146.6 & NA \\
\hline
\end{tabular}

Study Discussion and Technical Recommendations

The following recommendations are based on results obtained from the participants in this study.

$C B C$

- Approximately $19 \%$ of the laboratories reporting results for $\mathrm{CBC}$ provided values outside the consensus ranges for both Hemp Oil 1 and Hemp Oil 2 (Figure 4-4).

- Laboratories reporting results above the consensus mean in Hemp Oil 1 did not necessarily report results above the consensus mean for Hemp Oil 2. Trends of this type often represent potential sample interferences and miss identifications as illustrated in Section 1 for $\mathrm{CBC}$.

- The between-laboratory variability for CBC was higher in Hemp Oil 2a (6.0 \%) than Hemp Oil 1 or Hemp Oil $2(\approx 1.5 \%)$. The variability of individual mean laboratory means was higher for CBC in Hemp Oil 1 (6.7 \%) in comparison to Hemp Oil 2 (3.3\%) and Hemp Oil 2a (2.0 \%).

- Hemp Oil 2a was prepared through a methanol/ethanol extraction of Hemp Oil 2 at NIST. The additional processing of Hemp Oil $2 \mathrm{a}$ was expected to result in minimal variability both within and among participating laboratories.

- The elevated level of variability may be an artifact of significantly fewer laboratories reporting results for Hemp Oil 2a (11) compared to Hemp Oil 1 (53) and Hemp Oil 2 (59).

- NIST also observed a significant interference in the chromatographic peak of CBC as discussed in Section 1. Modification of the detection wavelength to $230 \mathrm{~nm}$ eliminated most of the 
interference; however, presence of a small interference is possible and may result in high bias of the NIST target value in comparison to the results obtained by other LC-UV and LC-PDA methods using different separation parameters. No trends for sample preparation were observed to explain the low recovery of $\mathrm{CBC}$ relative to the NIST value.

- No additional trends were observed for the sample preparation, analytical methods, and/or analytical parameters reported for $\mathrm{CBC}$ in the three hemp oil samples.

$C B C A$

- Most laboratories reported that CBCA was present in the samples at or below their LOQ (nonzero values). The low levels resulted in large consensus ranges and between-laboratory variabilities $(21 \%$ to $56 \%)$.

- Approximately $3 \%$ of the laboratories reporting results used LC-MS or LC-MS/MS methods did not have low enough LOQs to determine CBCA at the consensus levels in Hemp Oil 1, Hemp Oil 2, and Hemp Oil 2a.

- Approximately $93 \%$ of the laboratories reporting results used LC-UV or LC-PDA methods with only $5 \%, 5 \%$, and $33 \%$ of these laboratories with low enough LOQs to determine CBCA at the consensus levels in Hemp Oil 1, Hemp Oil 2, and Hemp Oil 2a, respectively.

- No additional trends were observed for the sample preparation, analytical methods, and/or analytical parameters reported for CBCA in the three hemp oil samples.

Overall

- Proper storage conditions are important for hemp oils because CBCA can readily convert to $\mathrm{CBC}$ when stored at elevated or room temperatures.

- Participants were asked to store the samples under controlled refrigeration $\left(\approx 4{ }^{\circ} \mathrm{C}\right)$.

- Laboratories should perform in-house evaluations of their storage conditions through stability test of their hemp oils at various environmental factors such as temperature, humidity, and light exposure. ${ }^{5}$

- Over 100 cannabinoids have been identified in Cannabis plant samples with similarities in structure and molecular mass. As a result, chromatographic peak identity should always be confirmed using appropriate reference spectra of pure standards for cannabinoids and is particularly important for cannabinoids that are present at levels close to the method LOQ. Analytical methods should be able to clearly distinguish between cannabinoids.

- The use of appropriate calibration materials and quality assurance samples to establish that a method is in control and being performed correctly may reduce the likelihood of outlying data. Quality assurance samples can be commercially available reference materials (CRMs, SRMs, or RMs) or materials prepared in-house.

- Measurement results should be reported accurately.

- Reported values should be the mass fraction (\%) of the CBC and CBCA in the three hemp oil samples. Participants who reported values based on a volume fraction (\%) for Hemp Oil 2 or Hemp Oil 2 a should use the density of the sample for the conversion to mass fraction. Hemp Oil 1 was too viscous to prepare dilutions by volume.

- Zero is not a quantity that can be measured. If values are below LOQ, results should be reported as such. A more appropriate result would be to report that a value is below the LOQ (e.g., " < 0.02 ”).

- Laboratories reporting results based on a certain threshold should enter the numerical threshold (e.g., "< 1"). 
- Laboratories reporting results flagged as outliers should check for calculation errors when preliminary data tables are sent for inspection. One example is to confirm that factors for all dilutions have been properly tabulated or that results are reported in the requested units. 
Table 4-1. Individualized data summary table (NIST) for CBC and CBCA in hemp oils.

\section{National Institute of Standards and Technology}

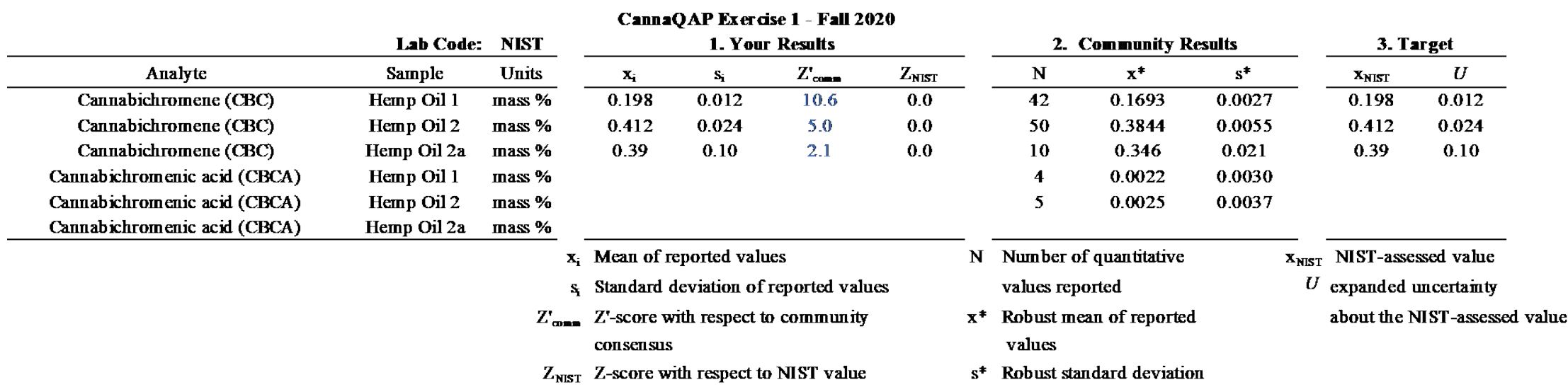


Table 4-2. Data summary table for $\mathrm{CBC}$ in hemp oils. Data highlighted in red have been flagged as a data entry of zero or results that include text (e.g., "< LOQ" or "present"). Data highlighted in blue have been identified as outside the consensus tolerance limits and would be estimated to result in an unacceptable $Z_{\text {comm }}^{\prime}$ score, $\left|Z_{\text {comm }}^{\prime}\right| \geq 2$. Note: This table spans two pages; the NIST values and consensus values are included on both pages for convenience.

\begin{tabular}{|c|c|c|c|c|c|c|c|c|c|c|c|c|c|c|c|c|}
\hline & \multirow[b]{3}{*}{ Lab } & \multicolumn{15}{|c|}{ Cannabihromene (CBC) } \\
\hline & & \multicolumn{5}{|c|}{ Heap Oil 1 (mass \%) } & \multicolumn{5}{|c|}{ Heap OI 2 (mass \%) } & \multicolumn{5}{|c|}{ Help OA 27 (mass \%) } \\
\hline & & $\mathbf{A}$ & $\mathbf{B}$ & $\mathbf{C}$ & Avg & SD & $\mathbf{A}$ & $\mathbf{B}$ & $\mathbf{C}$ & Avg & SD & $\mathbf{A}$ & $\mathbf{B}$ & $\mathbf{C}$ & Avg & SD \\
\hline \multirow{42}{*}{ 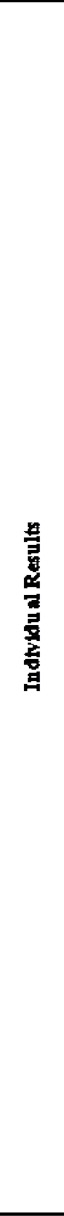 } & NIST & & & & $0 \_198$ & 0.012 & & & & 0.412 & 0.024 & & & & 0390 & 0.100 \\
\hline & $\mathrm{A} 002$ & 0.1695 & 0.17934 & 0.17043 & 0.1731 & 0.0054 & 0.393342 & 0.395283 & 0.368675 & 0.3858 & 0.0148 & & & & & \\
\hline & A003 & & & & & & 0.423 & 0.446 & 0.425 & 0.4313 & 0.0127 & & & & & \\
\hline & $\mathrm{A} 005$ & 0.164 & 0.16 & 0.177 & 0.1670 & 0.0089 & 0.411 & 0.405 & 0.408 & 0.4080 & 0.0030 & & & & & \\
\hline & A006 & 0.18 & & & 0.1800 & & 0.4 & & & 0.4000 & & & & & & \\
\hline & A 007 & & & & & & 0.31 & 0.33 & 0.31 & 0.3167 & 0.0115 & & & & & \\
\hline & A 008 & 0.18 & & & 0.1800 & & & & & & & & & & & \\
\hline & A 009 & & & & & & & & & & & 0.176 & 0.176 & 0.175 & 0.176 & 0.001 \\
\hline & A 010 & & & & & & & & & & & present & present & present & & \\
\hline & $\mathrm{A} 012$ & & & & & & 0.45956 & 0.45851 & 0.45935 & 0.4591 & 0.0006 & & & & & \\
\hline & A013 & 0.171 & 0.176 & 0.182 & 0.1763 & 0.0055 & 0378 & 0.414 & 0.413 & 0.4017 & 0.0205 & & & & & \\
\hline & A 014 & 0.17 & & & 0.1700 & & 0.42 & & & 0.4200 & & & & & & \\
\hline & A015 & & & & & & & & & & & 0.4 & 0.42 & 038 & 0.400 & 0.020 \\
\hline & A 016 & & & & & & & & & & & & & & & \\
\hline & A017 & 0.18 & & & 0.1800 & & 0.4 & & & 0.4000 & & & & & & \\
\hline & A 018 & & & & & & & & & & & & & & & \\
\hline & A019 & 0.17 & & & 0.1700 & & 038 & & & 03800 & & & & & & \\
\hline & $\mathrm{A} 020$ & 0.1763 & 0.178381 & 0.174015 & 0.1762 & 0.0022 & 0.371925 & 0.377754 & 0.422814 & 0.3908 & 0.0279 & & & & & \\
\hline & A021 & & & & & & & & & & & 031 & 0.35 & 034 & 0333 & 0.021 \\
\hline & $\mathrm{A} 022$ & & & & & & & & & & & 0.3335 & 0.3361 & 0.3375 & 0.336 & 0.002 \\
\hline & $\mathrm{A} 023$ & & & & & & 0.5182 & 0.5129 & 0.505 & 0.5120 & 0.0066 & & & & & \\
\hline & A 024 & & & & & & & & & & & 0.389 & 0.398 & 0.388 & 0.392 & 0.006 \\
\hline & A025 & & & & & & 036 & 0.26 & 0.4 & 0.3400 & 0.0721 & & & & & \\
\hline & A 027 & & & & & & & & & & & 0.375 & 0.378 & 0.38 & 0.378 & 0.003 \\
\hline & A030 & 0.2 & & & 0.2000 & & 0.46 & & & 0.4600 & & & & & & \\
\hline & $\mathrm{A} 031$ & & & & & & & & & & & & & & & \\
\hline & A033 & 0.16 & 0.14 & 0.16 & 0.1533 & 0.0115 & 036 & 0.36 & 036 & 0.3600 & 0.0000 & & & & & \\
\hline & A 035 & 0.169 & & & 0.1690 & & 0.37 & 0.373 & 0.372 & 0.3717 & 0.0015 & & & & & \\
\hline & A036 & 0.168 & 0.17 & 0.172 & 0.1700 & 0.0020 & 0388 & 0384 & 0.392 & 0.3880 & 0.0040 & & & & & \\
\hline & A 037 & 0.143 & 0.143 & 0.14 & 0.1420 & 0.0017 & 0.336 & 0.336 & 0.331 & 0.3343 & 0.0029 & & & & & \\
\hline & A038 & $0 \_167$ & 0.17 & 0.169 & 0.1687 & 0.0015 & 0373 & 0377 & 0.372 & 0.3740 & 0.0026 & & & & & \\
\hline & A 039 & 0.08 & 0.06 & 0.08 & 0.0733 & 0.0115 & 0.1 & 0.1 & 0.1 & 0.1000 & 0.0000 & & & & & \\
\hline & A040 & & & & & & & & & & & 031861 & 0.31907 & 031619 & 0318 & 0.002 \\
\hline & A 043 & 0.165 & 0.167 & 0.168 & 0.1667 & 0.0015 & 0.359 & 0.367 & 0.378 & 0.3680 & 0.0095 & & & & & \\
\hline & A044 & & & & & & & & & & & & & & & \\
\hline & A 045 & & & & & & & & & & & & & & & \\
\hline & A046 & 0.1 & 0.1 & 02 & 0.1333 & 0.0577 & 035 & 0.35 & 036 & 0.3533 & 0.0058 & & & & & \\
\hline & $\mathrm{A} 050$ & 0.186 & 0.187 & 0.187 & 0.1867 & 0.0006 & 0.387 & 0.392 & 0.39 & 0.3897 & 0.0025 & & & & & \\
\hline & A052 & & & & & & & & & & & & & & & \\
\hline & A 053 & & & & & & & & & & & & & & & \\
\hline & A054 & 0.17 & 0.17 & 0.17 & 0.1700 & 0.0000 & 039 & 0.37 & 037 & 0.3767 & 0.0115 & & & & & \\
\hline & $\mathrm{A} 055$ & 0.1553 & 0.1588 & 0.1535 & 0.1559 & 0.0027 & 0.3895 & 0.3929 & 0.3781 & 0.3868 & 0.0078 & & & & & \\
\hline 害等 & & $\begin{array}{l}\text { Consensu } \\
\text { Consensu } \\
\text { Maximum } \\
\text { Minimum } \\
\mathrm{N}\end{array}$ & $\begin{array}{l}\text { is Mean } \\
\text { ss Standard } \\
\text { a }\end{array}$ & Deviation & $\begin{array}{c}0.1693 \\
0.0027 \\
0.2400 \\
0.0000 \\
42\end{array}$ & & $\begin{array}{l}\text { Consensu } \\
\text { Consensu } \\
\text { Maximurm } \\
\text { Minimum } \\
\mathrm{N}\end{array}$ & $\begin{array}{l}\text { is Mean } \\
\text { is Standard } \\
\text { a }\end{array}$ & Deviation & $\begin{array}{c}0.3844 \\
0.0055 \\
0.5167 \\
0.0000 \\
50\end{array}$ & & $\begin{array}{l}\text { Consensu: } \\
\text { Consensu: } \\
\text { Maximum } \\
\text { Minimum } \\
\text { N }\end{array}$ & $\begin{array}{l}\text { Mean } \\
\text { Standand }\end{array}$ & Deviation & $\begin{array}{c}0.346 \\
0.021 \\
0.450 \\
0.176 \\
10\end{array}$ & \\
\hline
\end{tabular}




\begin{tabular}{|c|c|c|c|c|c|c|c|c|c|c|c|c|c|c|c|c|c|}
\hline & & & & & & & & & Cannab & ihroenene & (CBC) & & & & & & \\
\hline & & & & He=p & Oil 1 (nas & 5\%) & & & He=p & $072(\mathrm{mas}$ & 5 96) & & & He=p & 0120 (1 & 5 \%) & \\
\hline & & Lab & $\mathbf{A}$ & $\mathbf{B}$ & $\mathbf{C}$ & Avg & SD & $\mathbf{A}$ & B & $\mathbf{C}$ & Avg & SD & $\mathbf{A}$ & $\mathbf{B}$ & $\mathbf{C}$ & Avg & SD \\
\hline & & $\begin{array}{l}\text { NIST } \\
\text { A057 }\end{array}$ & & & & 0.198 & 0.012 & & & & 0.412 & 0024 & & & & 0390 & 0.100 \\
\hline & & A 058 & 0.304 & 0.189 & 0.192 & 0.2283 & 0.0655 & 0.387 & 0.434 & 0.6 & 0.4737 & 0.1119 & & & & & \\
\hline & & A059 & 0.202 & 0.2 & 0.201 & 0.2010 & 0.0010 & 0393 & 0387 & 0.388 & 0.3893 & 0.0032 & & & & & \\
\hline & & A 060 & 0.16 & 0.16 & 0.16 & 0.1600 & 0.0000 & 0.37 & 0.37 & 0.38 & 0.3733 & 0.0058 & & & & & \\
\hline$\sqrt{2}$ & & A061 & 0.169 & 0.169 & 0.168 & 0.1687 & 0.0006 & 0378 & 0368 & 0.375 & 0.3737 & 0.0051 & & & & & \\
\hline $\bar{n}$ & & A 062 & & & & & & & & & & & 0.355 & 0.356 & 0.368 & 0.360 & 0.007 \\
\hline 0 & & A063 & & & & & & 0.3305 & 0.33081 & 032983 & 0.3304 & 0.0005 & & & & & \\
\hline$E$ & & A 064 & & & & & & & & & & & & & & & \\
\hline$\underline{E}$ & & A066 & yes & yes & yes & & & & & & & & & & & & \\
\hline$\vec{\Omega}$ & & A 068 & & & & & & & & & & & & & & & \\
\hline$\stackrel{2}{\Rightarrow}$ & & A 071 & 0 & & & 00000 & & 0 & & & 00000 & & & & & & \\
\hline c & & A 072 & 0.17 & & & 0.1700 & & 0.36 & & & 0.3600 & & & & & & \\
\hline 追 & & A073 & 0.185 & 0.185 & 0.185 & 0.1850 & 0.0000 & 0.394 & 0392 & 0.389 & 0.3917 & 0.0025 & & & & & \\
\hline$\vec{\sim}$. & & A 074 & 0.144 & 0.134 & 0.151 & 0.1430 & 0.0085 & 0.356 & 0.368 & 0.357 & 0.3603 & 0.0067 & & & & & \\
\hline 0 & & A075 & & & & & & & & & & & & & & & \\
\hline$\sum$ & & A 076 & 0.006043 & 0.006147 & 0.006115 & 0.0061 & 0.0001 & 0.011866 & 0.011895 & 0.011648 & 0.0118 & 0.0001 & & & & & \\
\hline ڤِ & & $A 077$ & & & & & & $<\mathrm{IOQ}$ & $<\mathrm{IOQ}$ & $<\mathrm{LOQ}$ & & & & & & & \\
\hline D2 & & A 081 & 0.187 & 0.185 & 0.183 & 0.1850 & 0.0020 & 0.404 & 0.404 & 0.404 & 0.4040 & 0.0000 & & & & & \\
\hline$\underline{\sigma}$ & & A082 & & & & & & & & & & & 036 & & & 0360 & \\
\hline C & & A 083 & 0.17 & & & 0.1700 & & 0.38 & & & 0.3800 & & & & & & \\
\hline 三 & & A084 & 0.179 & 0.178 & 0.176 & 0.1777 & 0.0015 & 0398 & 0397 & 0.398 & 0.3977 & 0.0006 & & & & & \\
\hline$D^{\circ}$ & 告 & A 085 & 0.24 & 0.24 & 0.24 & 0.2400 & 0.0000 & 0.51 & 0.52 & 0.52 & 0.5167 & 0.0058 & & & & & \\
\hline 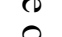 & 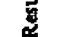 & A087 & & & & & & & & & & & & & & & \\
\hline$\stackrel{0}{\rightarrow}$ & $\frac{2}{7}$ & A 088 & & & & & & 0.37 & 0.37 & 0.38 & 0.3733 & 0.0058 & & & & & \\
\hline$\Omega$ & 寻 & A089 & 0.18 & 0.18 & 0.14 & 0.1667 & 0.0231 & 037 & 0.37 & 0.29 & 0.3433 & 0.0462 & & & & & \\
\hline $\overrightarrow{0}$ & $\frac{2}{8}$ & A 090 & & & & & & & & & & & 0.2132 & 0.2114 & 0.2143 & 0.213 & 0.001 \\
\hline & 韋 & A091 & & & & & & & & & & & & & & & \\
\hline da & & A 092 & 0.1549 & 0.1539 & 0.154 & 0.1543 & 0.0006 & 0.3553 & 0.3561 & 0.3575 & 0.3563 & 0.0011 & & & & & \\
\hline$\Rightarrow$ & & A093 & 0.17 & 0.18 & 0.17 & 0.1733 & 0.0058 & 036 & 0.36 & 0.37 & 0.3633 & 0.0058 & & & & & \\
\hline & & A 095 & 0.1475 & 0.1449 & 0.149 & 0.1471 & 0.0021 & 0.3601 & 0.3579 & 0.3524 & 0.3568 & 0.0040 & & & & & \\
\hline $\bar{\Xi}$ & & A096 & & & & & & & & & & & & & & & \\
\hline$\because$ & & A 098 & 0.177 & & & 0.1770 & & 0.42 & & & 0.4200 & & & & & & \\
\hline$\underline{\Xi}$ & & A099 & 0.16 & 0.163 & 0.166 & 0.1630 & 0.0030 & 0372 & 0372 & 0.369 & 0.3710 & 0.0017 & & & & & \\
\hline & & A 100 & 0.1923 & 0.1956 & 0.1954 & 0.1944 & 0.0019 & 0.4543 & 0.4464 & 0.4385 & 0.4464 & 0.0079 & & & & & \\
\hline 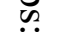 & & A 101 & & & & & & & & & & & & & & & \\
\hline$\ddot{\rightleftharpoons}$ & & A 102 & 0.17 & 0.17 & 0.17 & 0.1700 & 0.0000 & 0.39 & 0.39 & 0.39 & 0.3900 & 0.0000 & & & & & \\
\hline ?당 & & A 103 & 0 & 0 & 0 & 00000 & 0.0000 & 0299 & 0398 & 0.404 & 0.3670 & 0.0590 & & & & & \\
\hline & & A 104 & 0.168 & 0.169 & 0.17 & 0.1690 & 0.0010 & 0.386 & 0.377 & 0.381 & 0.3813 & 0.0045 & & & & & \\
\hline 0 & & A 105 & 0.16 & 0.15 & 0.16 & 0.1567 & 0.0058 & 0.4 & 0.39 & 039 & 0.3933 & 0.0058 & & & & & \\
\hline $\overrightarrow{00}$ & & A 106 & 0.16 & 0.16 & 0.157 & 0.1590 & 0.0017 & 0.392 & 0.376 & 0.369 & 0.3790 & 0.0118 & & & & & \\
\hline 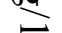 & & A 107 & 0.14 & 0.134 & 0.137 & 0.1370 & 0.0030 & 0313 & 0.299 & 029 & 0.3007 & 0.0116 & & & & & \\
\hline$c$ & & A 108 & & & & & & & & & & & & & & & \\
\hline a & & A 109 & 025 & 0.18 & & 0.2150 & 0.0495 & 0.53 & 0.47 & & 0.5000 & 0.0424 & & & & & \\
\hline 0 & & A 110 & 0.16 & 0.16 & 0.016 & 0.1120 & 0.0831 & 0.36 & 0.36 & 0.36 & 0.3600 & 0.0000 & & & & & \\
\hline$\infty$ & & A112 & 0.147 & 0.192 & 0.1695 & 0.1695 & 0.0225 & 0328 & 0368 & 0.348 & 0.3480 & 0.0200 & & & & & \\
\hline$\nabla$ & & A 113 & 0.135541 & 0.136351 & 0.136725 & 0.1362 & 0.0006 & 0.024787 & 0.024675 & 0.024704 & 0.0247 & 0.0001 & & & & & \\
\hline 1 & & A114 & 0.17 & 0.1715 & 0.1853 & 0.1756 & 0.0084 & 0.3872 & 03858 & 03861 & 0.3864 & 0.0007 & & & & & \\
\hline$\pi$ & & A 115 & & & & & & & & & & & 0.45 & 0.44 & 0.46 & 0.450 & 0.010 \\
\hline$\exists$ & & A116 & 0.0819 & 0.09 & 0.09 & 0.0873 & 0.0047 & 0.1826 & 0.24 & 0.221 & 0.2145 & 0.0292 & & & & & \\
\hline $\begin{array}{l}\not \\
\infty \\
\infty \\
\infty\end{array}$ & 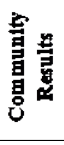 & & $\begin{array}{l}\text { Consensus } \\
\text { Consensus } \\
\text { Maximum } \\
\text { Minimum } \\
\mathrm{N}\end{array}$ & $\begin{array}{l}\text { s Mean } \\
\text { is Standard } 1\end{array}$ & Deviation & $\begin{array}{c}0.1693 \\
0.0027 \\
0.2400 \\
0.0000 \\
42 \\
\end{array}$ & & $\begin{array}{l}\text { Consensus } \\
\text { Consensus } \\
\text { Maximum } \\
\text { Minimum } \\
\text { N }\end{array}$ & $\begin{array}{l}\text { s Mean } \\
\text { s Standard I }\end{array}$ & Deviation & $\begin{array}{c}0.3844 \\
0.0055 \\
0.5167 \\
0.0000 \\
50 \\
\end{array}$ & & $\begin{array}{l}\text { Consensus } \\
\text { Consensus } \\
\text { Maximum } \\
\text { Minimum } \\
\text { N }\end{array}$ & $\begin{array}{l}\text { Mean } \\
\text { Standand }\end{array}$ & eviation & $\begin{array}{c}0.346 \\
0.021 \\
0.450 \\
0.176 \\
10 \\
\end{array}$ & \\
\hline
\end{tabular}




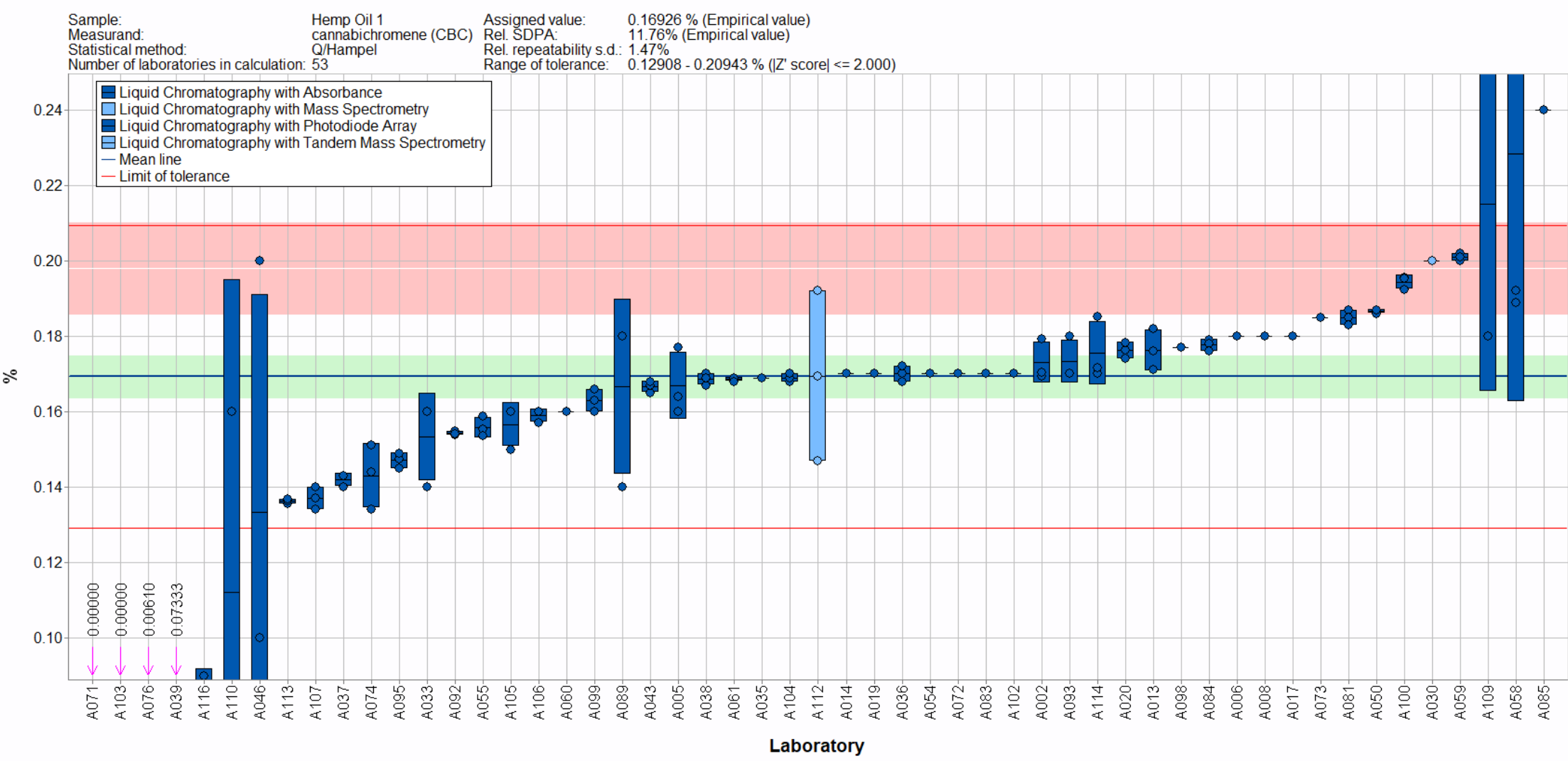

Figure 4-1. CBC in Hemp Oil 1 (data summary view - analytical method). In this view, individual laboratory data are plotted (circles) with the individual laboratory standard deviation (rectangle). The color of the data point represents the analytical method employed. The solid blue line represents the consensus mean, and the green shaded region represents the $95 \%$ confidence interval for the consensus mean. The solid red lines represent the consensus range of tolerance, calculated as the values above and below the consensus mean that result in an acceptable $Z_{\text {comm }}^{\prime}$ score, $\left|Z_{\text {comm }}^{\prime}\right| \leq 2$. The red shaded region represents the NIST range of tolerance, which encompasses the target value bounded by twice its uncertainty ( $U_{\text {NIST }}$ ) and represents the range that results in an acceptable $Z_{\text {NIST }}$ score, $\left|Z_{\text {NIST }}\right| \leq 2$. The shaded beige region represents the overlapping of the $95 \%$ confidence interval for the consensus mean (green region) and the NIST range of tolerance (red region). 


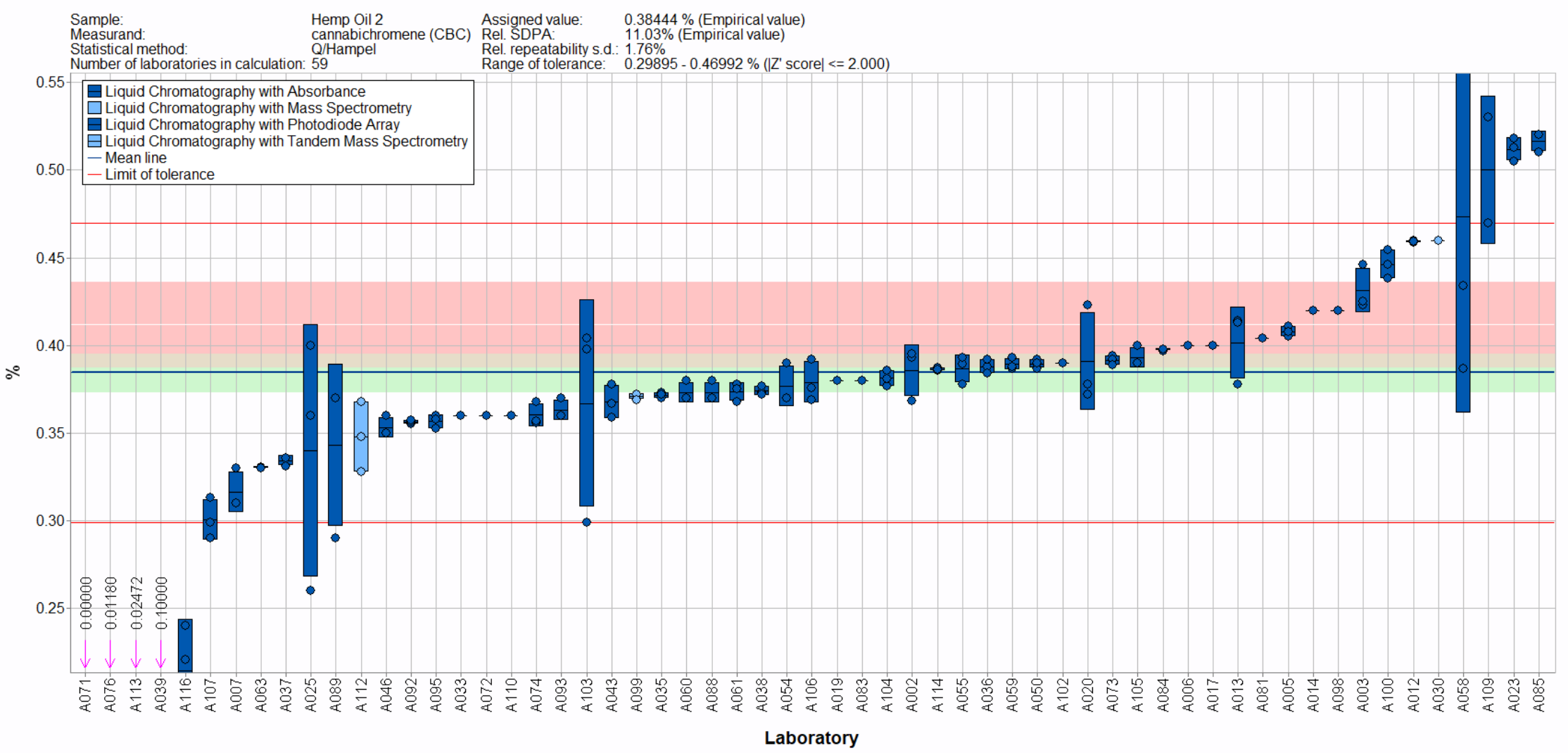

Figure 4-2. CBC in Hemp Oil 2 (data summary view - analytical method). In this view, individual laboratory data are plotted (circles) with the individual laboratory standard deviation (rectangle). The color of the data point represents the analytical method employed. The solid blue line represents the consensus mean, and the green shaded region represents the $95 \%$ confidence interval for the consensus mean. The solid red lines represent the consensus range of tolerance, calculated as the values above and below the consensus mean that result in an acceptable $Z_{\text {comm }}^{\prime}$ score, $\left|Z_{\text {comm }}^{\prime}\right| \leq 2$. The red shaded region represents the NIST range of tolerance, which encompasses the target value bounded by twice its uncertainty ( $U_{\text {NIST }}$ ) and represents the range that results in an acceptable $Z_{\text {NIST }}$ score, $\left|Z_{\text {NIST }}\right| \leq 2$. The shaded beige region represents the overlapping of the $95 \%$ confidence interval for the consensus mean (green region) and the NIST range of tolerance (red region). 


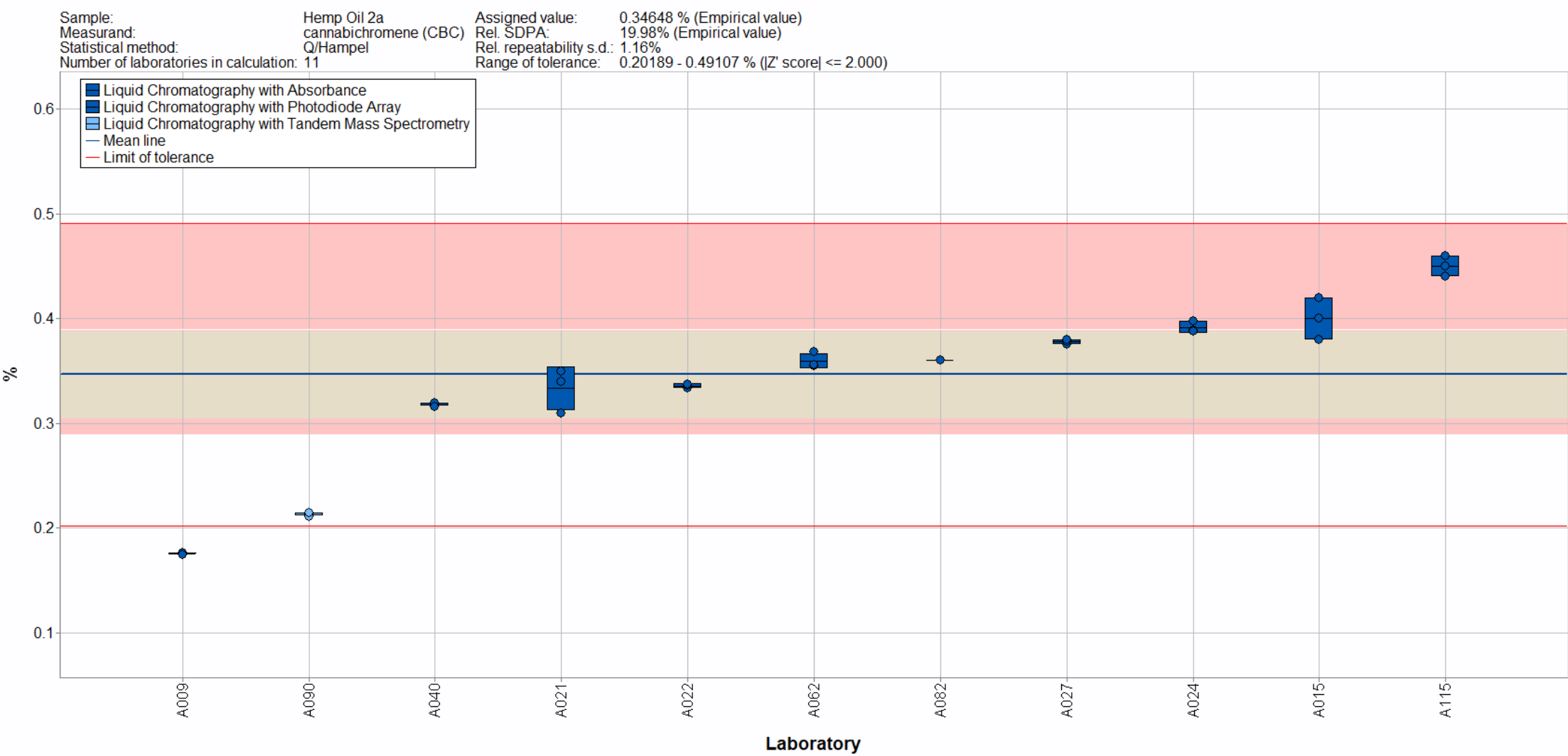

Figure 4-3. CBC in Hemp Oil 2a (data summary view - analytical method). In this view, individual laboratory data are plotted (circles) with the individual laboratory standard deviation (rectangle). The color of the data point represents the analytical method employed. The solid blue line represents the consensus mean, and the green shaded region represents the $95 \%$ confidence interval for the consensus mean. The solid red lines represent the consensus range of tolerance, calculated as the values above and below the consensus mean that result in an acceptable $Z_{\text {comm }}^{\prime}$ score, $\left|Z_{\text {comm }}^{\prime}\right| \leq 2$. The red shaded region represents the NIST range of tolerance, which encompasses the target value bounded by twice its uncertainty ( $U_{\text {NIST }}$ ) and represents the range that results in an acceptable $Z_{\text {NIST }}$ score, $\left|Z_{\text {NIST }}\right| \leq 2$. The shaded beige region represents the overlapping of the $95 \%$ confidence interval for the consensus mean (green region) and the NIST range of tolerance (red region). 


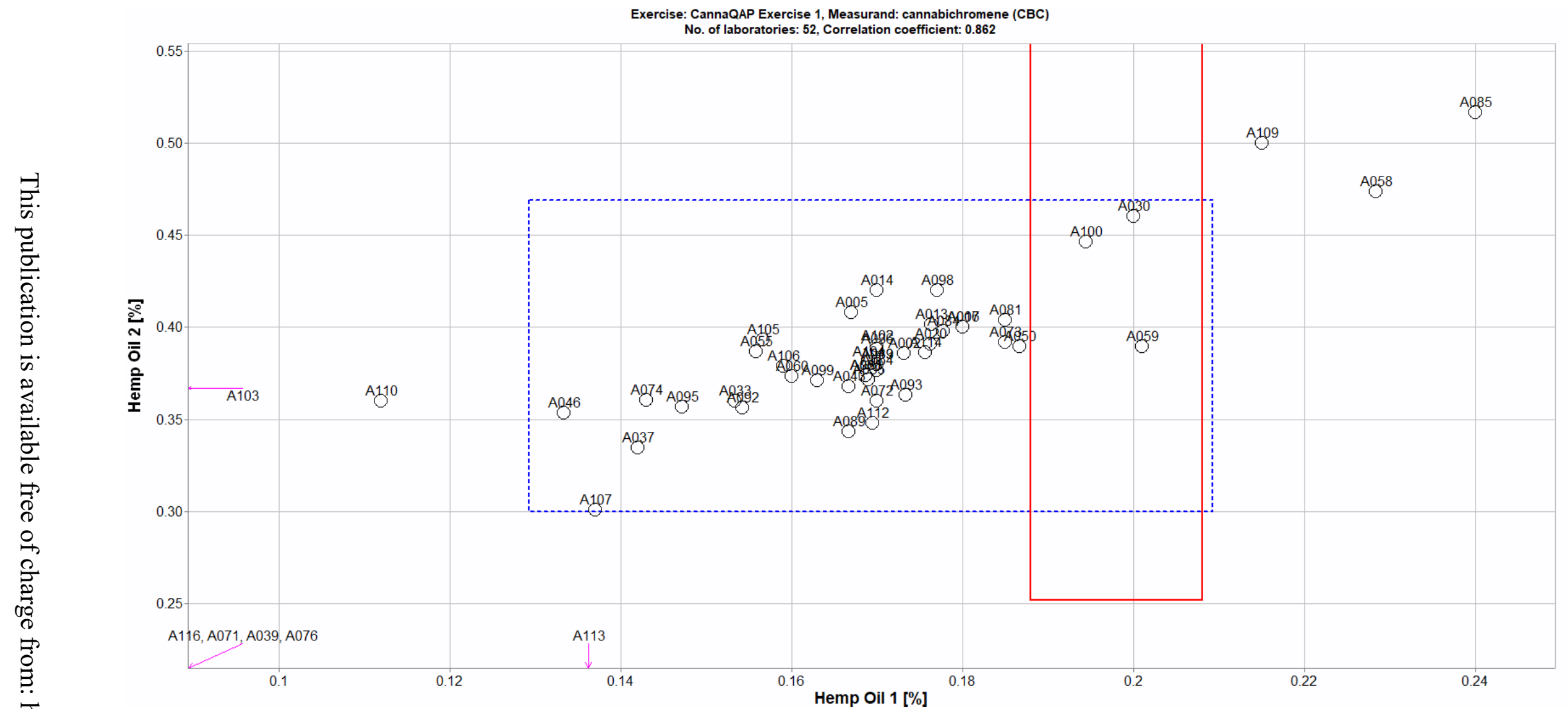

Figure 4-4. Laboratory means for CBC in Hemp Oil 1 and Hemp Oil 2 (sample/sample comparison view). In this view, the individual laboratory mean for one sample (Hemp Oil 1) is compared to the individual laboratory mean for a second sample (Hemp Oil 2). The solid red box represents the NIST range of tolerance for the two samples, Hemp Oil 1 (x-axis) and Hemp Oil 2 (y-axis), which encompasses the target values bounded by their uncertainties ( $\left.U_{\text {NIST }}\right)$ and represents the range that results in an acceptable $Z_{\text {NIST }}$ score, $\left|Z_{\text {NIST }}\right| \leq 2$. The dotted blue box represents the consensus range of tolerance for Hemp Oil 1 (x-axis) and Hemp Oil 2 (y-axis), calculated as the values above and below the consensus means that result in an acceptable $Z_{\text {comm }}^{\prime}$ score, $\left|Z_{\text {comm }}^{\prime}\right| \leq 2$. 
Table 4-3. Data summary table for CBCA in hemp oils. Data highlighted in red have been flagged as a data entry of zero or results that include text (e.g., "< LOQ" or "present").

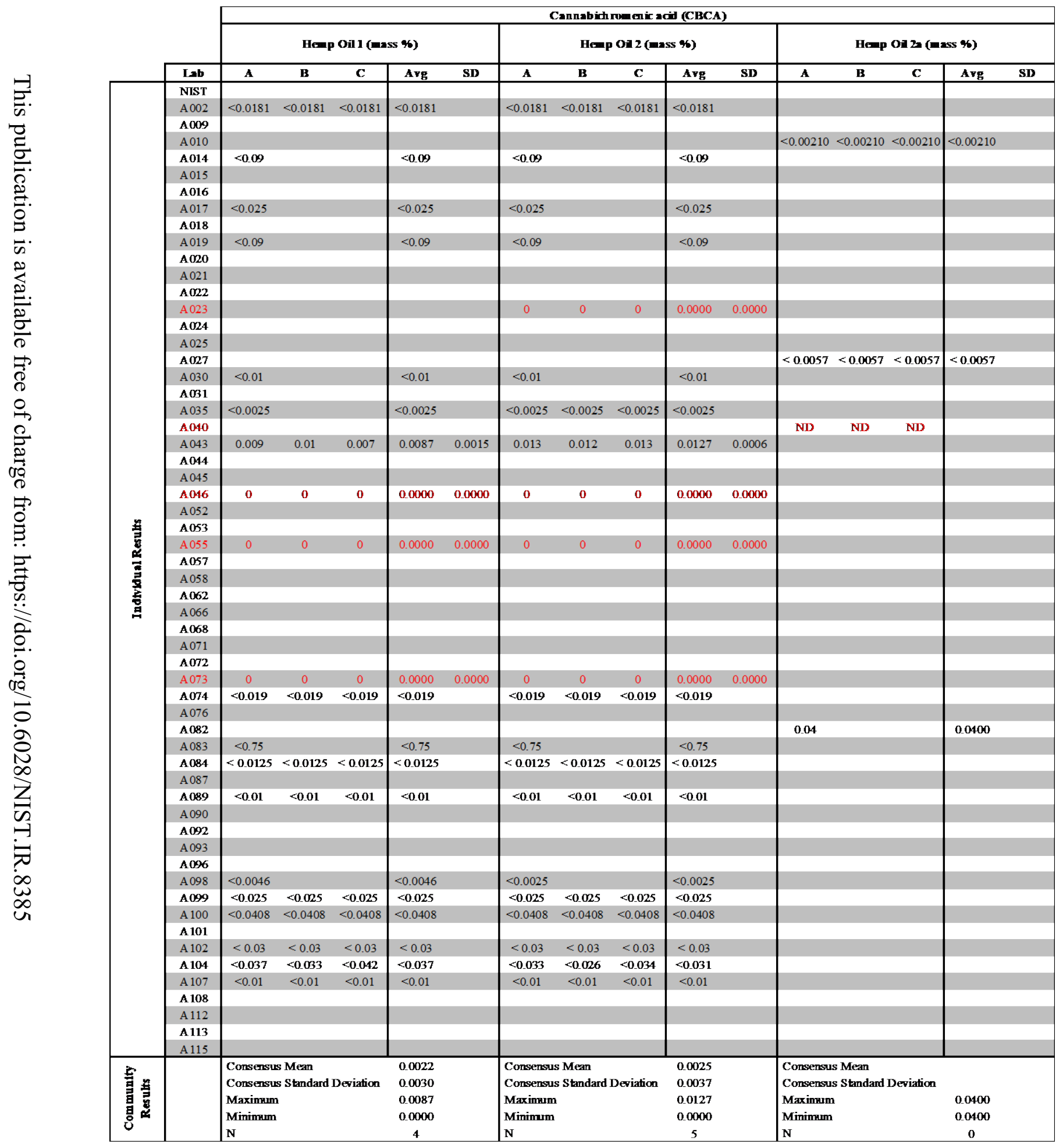




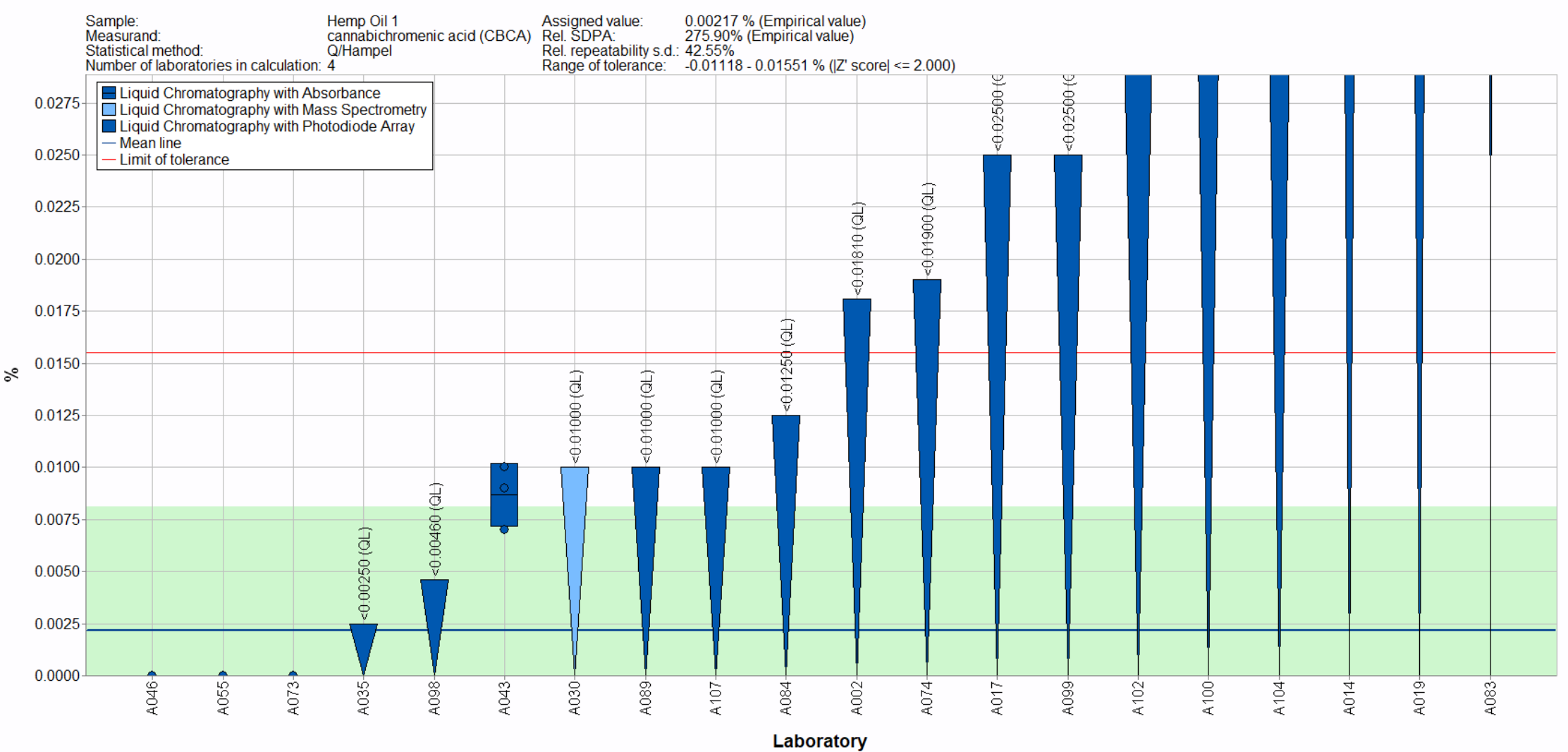

Figure 4-5. CBCA in Hemp Oil 1 (data summary view - analytical method). In this view, individual laboratory data are plotted (circles) with the individual laboratory standard deviation (rectangle). The color of the data point represents the analytical method employed. The solid blue line represents the consensus mean, and the green shaded region represents the $95 \%$ confidence interval for the consensus mean. The solid red line represents the upper consensus range of tolerance, calculated as the values above the consensus mean that results in an acceptable $Z_{\text {comm }}^{\prime}$ score, $\left|Z_{\text {comm }}^{\prime}\right| \leq 2$, with the lower limit set at zero. A NIST value has not been determined in this material. The downward triangle represents data reported as a threshold or LOQ value. 


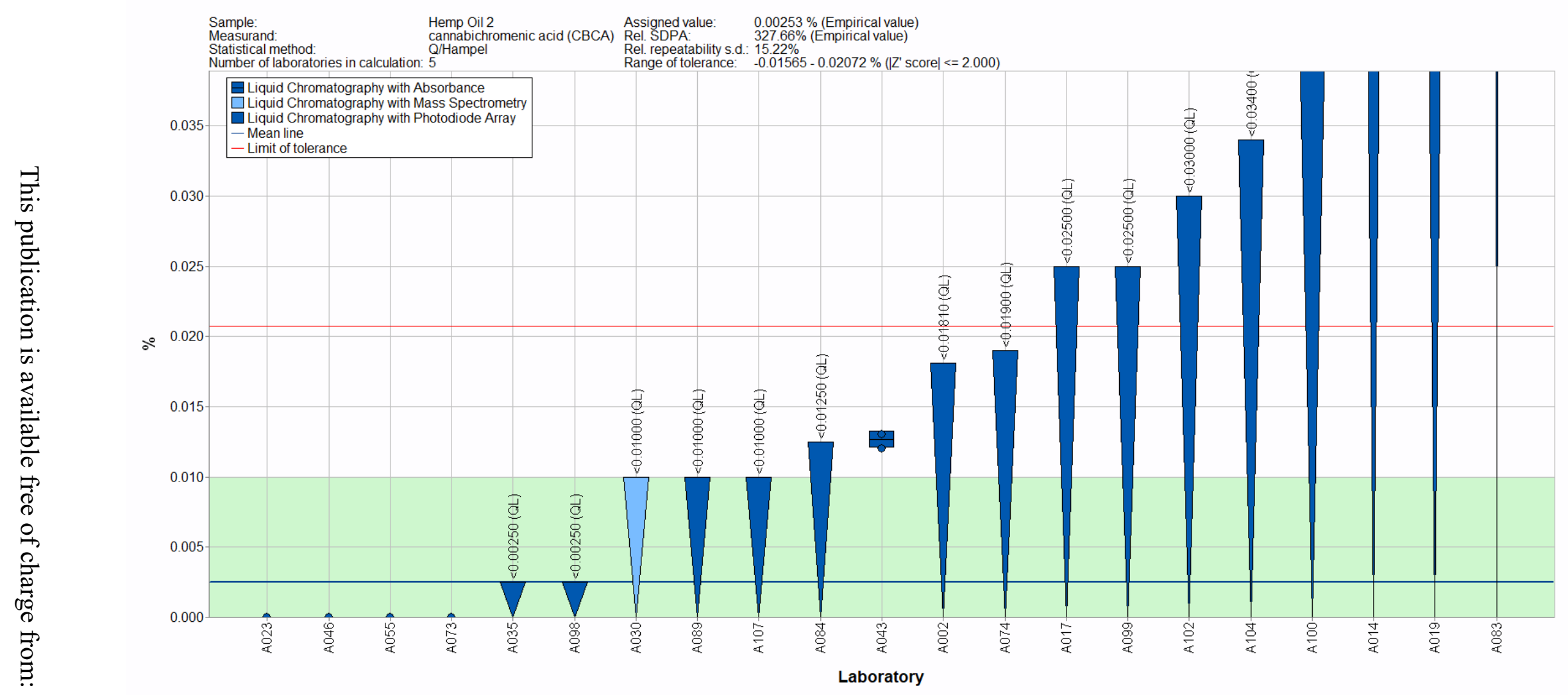

Figure 4-6. CBCA in Hemp Oil 2 (data summary view - analytical method). In this view, individual laboratory data are plotted (circles) with the individual laboratory standard deviation (rectangle). The color of the data point represents the analytical method employed. The solid blue line represents the consensus mean, and the green shaded region represents the $95 \%$ confidence interval for the consensus mean. The solid red line represents the upper consensus range of tolerance, calculated as the values above the consensus mean that results in an acceptable $Z_{\text {comm }}^{\prime}$ score, $\left|Z_{\text {comm }}^{\prime}\right| \leq 2$, with the lower limit set at zero. A NIST value has not been determined in this material. The downward triangle represents data reported as a threshold or LOQ value. 


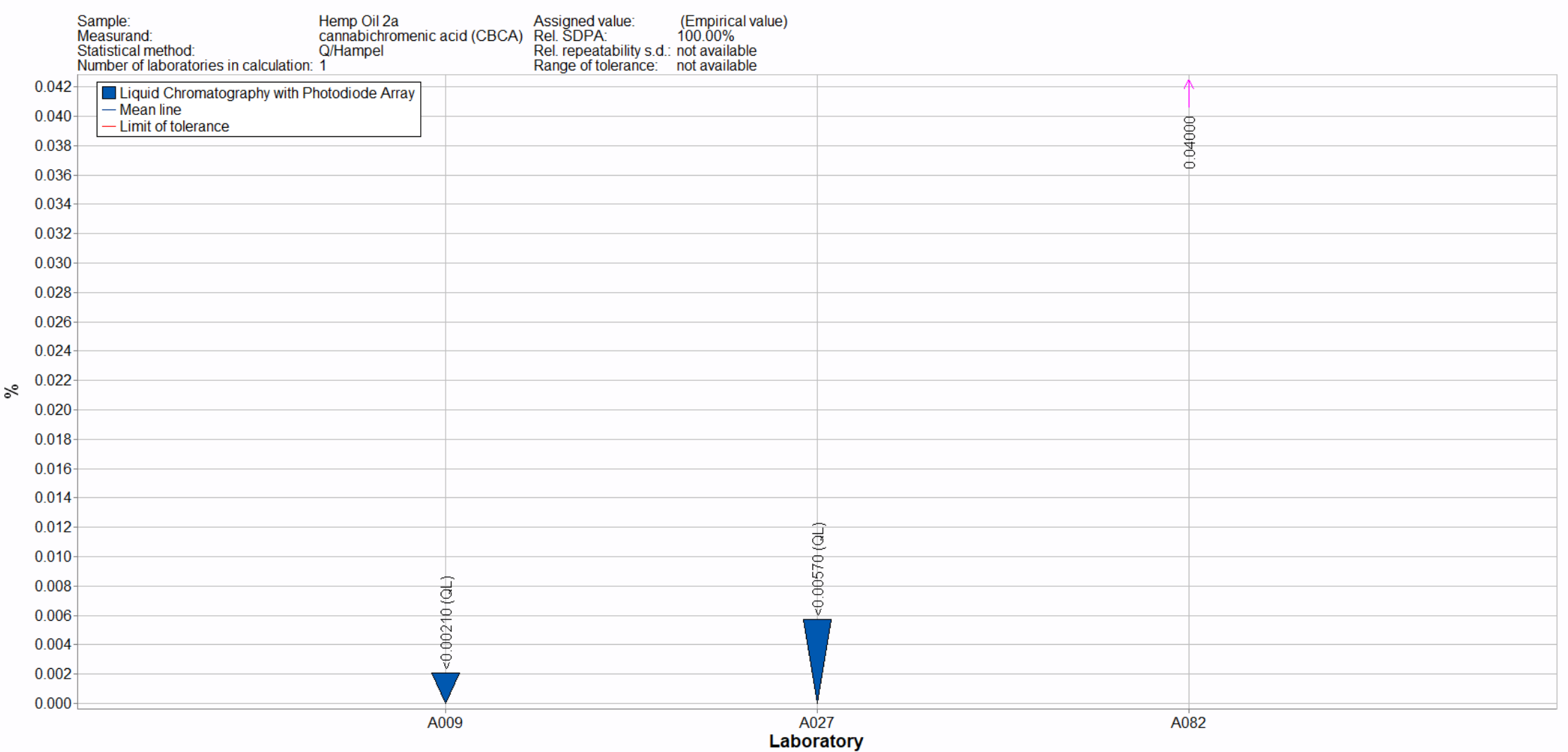

Figure 4-7. CBCA in Hemp Oil 2a (data summary view - analytical method). In this view, individual laboratory data are plotted (circles) with the individual laboratory standard deviation (rectangle). The color of the data point represents the analytical method employed. The downward triangle represents data reported as a threshold or LOQ value. 


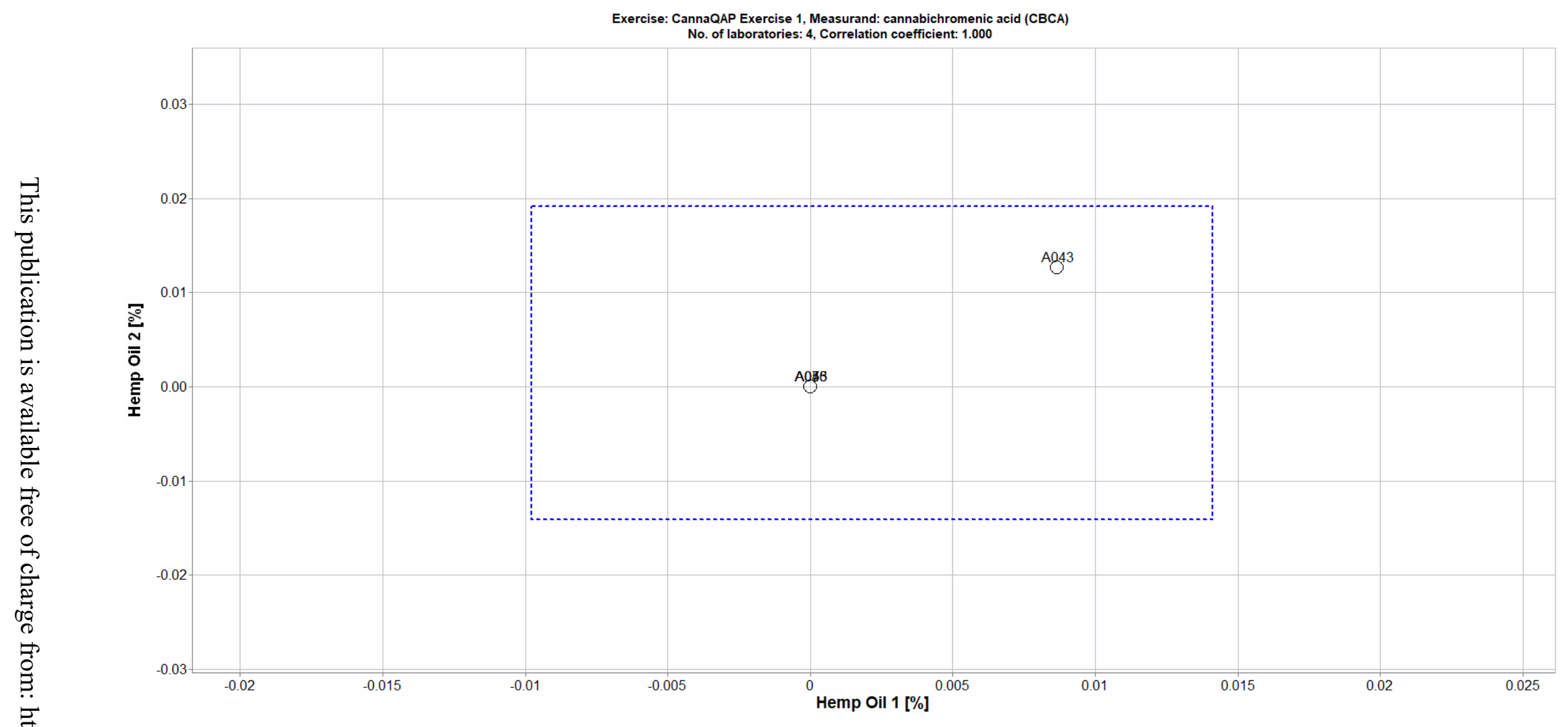

Figure 4-8. Laboratory means for CBCA in Hemp Oil 1 and Hemp Oil 2 (sample/sample comparison view). In this view, the individual laboratory mean for one sample (Hemp Oil 1) is compared to the individual laboratory mean for a second sample (Hemp Oil 2). The dotted blue box represents the consensus range of tolerance for Hemp Oil 1 (x-axis) and Hemp Oil 2 (y-axis), calculated as the values above and below the consensus means that result in an acceptable $Z_{\text {comm }}^{\prime}$ score, $\left|Z_{\text {comm }}^{\prime}\right| \leq 2$. 


\section{SECTION 5: CBDV AND CBDVA}

Study Overview

CBDV is a non-intoxicating cannabinoid often detected in Cannabis plants and Cannabis-derived products. CBDV is a homolog of CBD with an alternate side chain shortened by two methylene units. CBDV has attracted significant interest in the research community and reliable analytical methods are necessary to further explore potential health benefits. ${ }^{9} \mathrm{CBDV}$ does not exist in the Cannabis plant naturally but is formed through decarboxylation of its acidic precursor (CBDVA) by exposure to heat or light. Participants in this study were asked to use in-house analytical methods to determine the mass fraction (\%) of CBDV and CBDVA in the three hemp oils. The preparation of these hemp oils included a decarboxylation step resulting in extremely low levels of CBDVA and levels of CBDV consistent with normal ranges in commercial products.

\section{$\underline{\text { Reporting Statistics }}$}

- The enrollment and reporting statistics for CBDV and CBDVA are described in the table below for each analyte. Reported values may include non-quantitative results (zero or below LOQ).

\begin{tabular}{cccccccc} 
& \multicolumn{2}{c}{ Hemp Oil 1 } & \multicolumn{2}{c}{ Hemp Oil 2 } & & \multicolumn{2}{c}{ Hemp Oil 2a } \\
& $\underline{\text { Number of }}$ & $\underline{\underline{\text { Reporting }}}$ & & $\underline{\text { Number of }}$ & $\underline{\underline{\text { Reporting }}}$ & $\underline{\text { Number of }}$ & $\underline{\underline{\text { Reporting }}}$ \\
$\underline{\text { Analyte }}$ & $\underline{\underline{\text { Participants }}}$ & $\underline{\text { Results }}$ & $\underline{\text { Participants }}$ & $\underline{\text { Results }}$ & $\underline{\text { Participants }}$ & $\underline{\text { Results }}$ \\
CBDV & 56 & $82 \%$ & 64 & $78 \%$ & 19 & $63 \%$ \\
CBDVA & 38 & $61 \%$ & 40 & $63 \%$ & 19 & $21 \%$
\end{tabular}

- Most laboratories reported using solvent extraction or sample dilution for determination of CBDV and CBDVA in the three hemp oil samples (see table below). Additional sample preparation details are summarized at the end of the report in Appendix I.

\begin{tabular}{ccc}
$\begin{array}{c}\text { Reported } \\
\text { Preparation Method }\end{array}$ & CBDV & CBDVA \\
\cline { 1 - 2 } Solvent Extraction & 70.4 & 71.9 \\
Dilution & 24.3 & 25.0 \\
Other & 0.0 & 0.0 \\
None & 2.6 & 1.6 \\
No Response & 2.6 & 1.6
\end{tabular}

\footnotetext{
${ }^{9}$ N Stone, A Murphy, T England, S O'Sullivan. Br J Pharmacol. 177: 4330-4352 (2020) https://doi.org/10.1111/bph.15185.
} 
- Most laboratories reported using LC-PDA or LC-UV for the determination of CBDV and CBDVA in the three hemp oil samples (see table below). Additional method details are summarized at the end of the report in Appendix I.

\begin{tabular}{ccc}
$\begin{array}{c}\text { Reported } \\
\text { Analytical Method }\end{array}$ & & \multicolumn{2}{c}{ Percent Reporting } \\
\cline { 1 - 2 } LC-PDA & 64.3 & $\frac{\text { CBDVA }}{65.6}$ \\
LC-UV & 27.0 & 29.7 \\
LC-MS & 0.0 & 0.0 \\
LC-MS/MS & 5.2 & 1.6 \\
GC-FID & 0.0 & 0.0 \\
GC-MS & 2.6 & 0.0 \\
Other & 0.9 & 3.1
\end{tabular}

$\underline{\text { Study Results }}$

$C B D V$

- The mass fractions (\%) for CBDV in the hemp oil samples were determined by NIST using LC-PDA as described in Section 1 and are summarized in Table 5-1. These NIST values are used as the target means and ranges summarized in Table 5-2 for comparison to the participant results.

- The target and consensus means and ranges are summarized for CBDV via different analytical methods in Figure 5-1, Figure 5-2, and Figure 5-3, which include data from laboratories submitting two or three results for CBDV. Data from participants submitting only one measurement were included in Table 5-2 but were not included in the calculation of consensus statistics. $^{2}$

- $\quad$ For CBDV in Hemp Oil 1, the consensus range was based on quantitative results from 39 laboratories and completely overlaps with the target range (Figure 5-1).

- The individual laboratory means from 21 laboratories (54\% of those reporting results) were outside the NIST range of tolerance for CBDV in Hemp Oil 1.

- The individual laboratory means from 3 laboratories (8\% of those reporting results) were outside the acceptable $Z_{\text {comm }}^{\prime}$ score for CBDV in Hemp Oil 1.

- The thresholds or LOQs for 1 of 6 laboratories reporting qualitative values were below the target mean for CBDV in Hemp Oil 1.

- For CBDV in Hemp Oil 2, the consensus range was based on quantitative results from 49 laboratories and completely overlaps the target range (Figure 5-2).

- The individual laboratory means or thresholds from 26 laboratories (53\% of those reporting results) were outside the NIST range of tolerance for CBDV in Hemp Oil 2.

- The individual laboratory means from 7 laboratories (14\% of those reporting results) were outside the acceptable $Z_{\text {comm }}^{\prime}$ score for CBDV in Hemp Oil 2.

- No results were reported using thresholds or LOQs for CBDV in Hemp Oil 2.

- For CBDV in Hemp Oil 2a, the consensus range was based on quantitative results from 11 laboratories and overlaps $80 \%$ of the target range (Figure 5-3). 
- The individual laboratory means or thresholds from 6 laboratories $(55 \%$ of those reporting results) were outside the NIST range of tolerance for CBDV in Hemp Oil 2a.

- The individual laboratory mean from 1 laboratory ( $9 \%$ of those reporting results) was outside the acceptable $Z_{\text {comm }}^{\prime}$ score for CBDV in Hemp Oil $2 \mathrm{a}$.

- No results were reported using thresholds or LOQs for CBDV in Hemp Oil 2a.

- A comparison of individual laboratory means for CBDV in Hemp Oil 1 and Hemp Oil 2 is summarized in Figure 5-4 for laboratories who reported results for both samples.

$C B D V A$

- No target means or ranges were provided in Table 5-1 for CBDVA in the three hemp oils.

- The consensus means and ranges for CBDVA are based on quantitative data from 9 laboratories (Figure 5-5), 13 laboratories (Figure 5-6) for Hemp Oil 1 and Hemp Oil 2, respectively. A consensus mean could not be determined for CBDVA in Hemp Oil 2a (Figure 5-7). Data from participants submitting only one measurement were included in Table 5-3 but were not included in the calculation of consensus statistics. ${ }^{2}$

- A comparison of individual laboratory means for CBDVA in Hemp Oil 1 and Hemp Oil 2 is summarized in Figure 5-8 for laboratories who reported results for both samples.

Overall

- The between-laboratory variabilities for determination of CBDV and CBDVA in the hemp oil samples are shown in the table below.

Between-Laboratory Variability (\% RSD)

\begin{tabular}{|c|c|c|c|}
\hline Analyte & Hemp Oil 1 & Hemp Oil 2 & Hemp Oil 2a \\
\hline CBDV & 8.5 & 3.2 & 6.3 \\
\hline CBDVA & 69.3 & 34.9 & NA \\
\hline
\end{tabular}

Study Discussion and Technical Recommendations

The following recommendations are based on results obtained from the participants in this study.

$C B D V$

- Approximately $8 \%$ of the laboratories reporting results for CBDV provided values outside the consensus ranges for both Hemp Oil 1 and Hemp Oil 2 (Figure 5-4).

- Laboratories reporting results below the consensus mean in Hemp Oil 1 also reported results below the consensus mean for Hemp Oil 2. The reverse is also observed for laboratories reporting values above the consensus mean in both samples. Trends of this type often indicate a calibration bias.

- The between-laboratory variability was lower for CBDV in Hemp Oil 2 (3.2 \%) than Hemp Oil $1(8.5 \%)$ and Hemp Oil $2 \mathrm{a}(6.3 \%)$. The individual mean laboratory variability was lower for CBDV in Hemp Oil 2a (3.8\%) in comparison to Hemp Oil 1 (6.1\%) and Hemp Oil 2 $(6.1 \%)$.

- Hemp Oil 2a was prepared through a methanol/ethanol extraction of Hemp Oil 2 at NIST. The additional processing of Hemp Oil $2 \mathrm{a}$ was expected to result in minimal variability both within and among participating laboratories. 
- No additional trends were observed for the sample preparation, analytical methods, and/or analytical parameters reported for CBDV in the three hemp oil samples.

\section{$C B D V A$}

- Most laboratories reported that CBDVA was present in the samples at or below their LOQ (non-zero values). The low levels resulted in large consensus ranges and between-laboratory variabilities (35\% to $69 \%)$.

- All the laboratories reporting results used LC-UV or LC-PDA methods with only $17 \%$ and $28 \%$ of these laboratories with low enough LOQs to determine CBDVA at the consensus levels in Hemp Oil 1 and Hemp Oil 2. No laboratories using LC-UV or LC-PDA reported LOQs low enough to determine CBDVA at the consensus level in Hemp Oil 2a.

- No additional trends were observed for the sample preparation, analytical methods, and/or analytical parameters reported for CBDVA in the three hemp oil samples.

\section{Overall}

- Proper storage conditions are important for hemp oils because CBDVA can readily convert to CBDV when stored at elevated or room temperatures.

- Participants were asked to store the samples under controlled refrigeration $\left(\approx 4{ }^{\circ} \mathrm{C}\right)$.

- Laboratories should perform in-house evaluations of their storage conditions through stability test of their hemp oils at various environmental factors such as temperature, humidity, and light exposure. ${ }^{5}$

- Over 100 cannabinoids have been identified in Cannabis plant samples with similarities in structure and molecular mass. As a result, chromatographic peak identity should always be confirmed using appropriate reference spectra of pure standards for cannabinoids and is particularly important for cannabinoids that are present at levels close to the method LOQ. Analytical methods should be able to clearly distinguish between cannabinoids.

- The use of appropriate calibration materials and quality assurance samples to establish that a method is in control and being performed correctly may reduce the likelihood of outlying data. Quality assurance samples can be commercially available reference materials (CRMs, SRMs, or RMs) or materials prepared in-house.

- Measurement results should be reported accurately.

- Reported values should be the mass fraction (\%) of the CBDV and CBDVA in the three hemp oil samples. Participants who reported values based on a volume fraction (\%) for Hemp Oil 2 or Hemp Oil 2a should use the density of the sample for the conversion to mass fraction. Hemp Oil 1 was too viscous to prepare dilutions by volume.

- Zero is not a quantity that can be measured. If values are below LOQ, results should be reported as such. A more appropriate result would be to report that a value is below the LOQ (e.g., " $<0.02 ”)$.

- Laboratories reporting results based on a certain threshold should enter the numerical threshold (e.g., "< 1 ").

- Laboratories reporting results flagged as outliers should check for calculation errors when preliminary data tables are sent for inspection. One example is to confirm that factors for all dilutions have been properly tabulated or that results are reported in the requested units. 
Table 5-1. Individualized data summary table (NIST) for CBDV and CBDVA in hemp oils.

\section{National Institute of Standards and Technology}

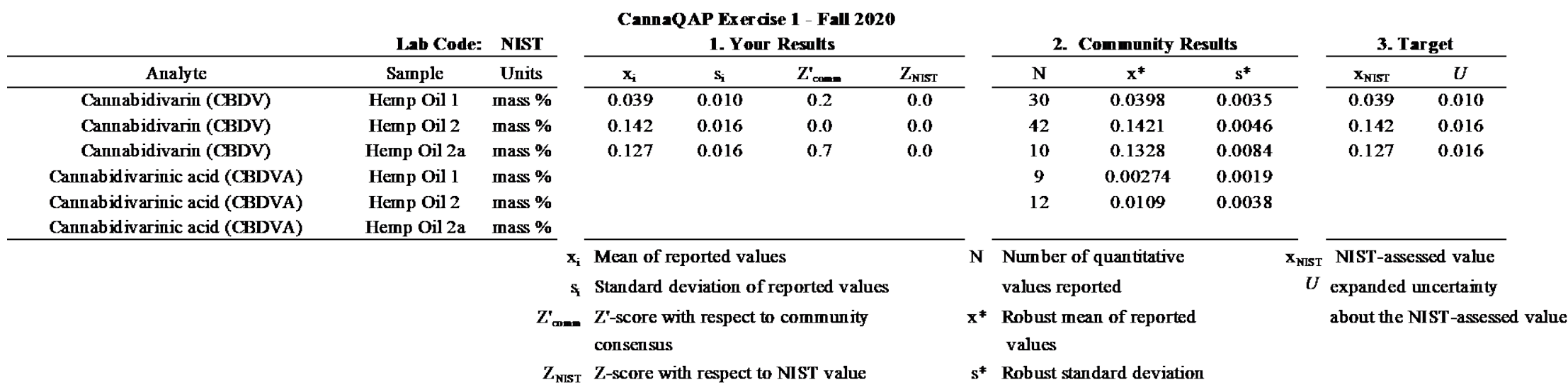


Table 5-2. Data summary table for CBDV in hemp oils. Data highlighted in red have been flagged as a data entry of zero or results that include text (e.g., "<LOQ" or "present"). Data highlighted in blue have been identified as outside the consensus tolerance limits and would be estimated to result in an unacceptable $Z_{\text {comm }}^{\prime}$ score, $\left|Z_{\text {comm }}^{\prime}\right| \geq 2$. Note: This table spans two pages; the NIST values and consensus values are included on both pages for convenience.

\begin{tabular}{|c|c|c|c|c|c|c|c|c|c|c|c|c|c|c|c|c|}
\hline & \multirow[b]{3}{*}{ Lab } & \multicolumn{15}{|c|}{ Cannabidivarin (CRDV) } \\
\hline & & \multicolumn{5}{|c|}{ He=p Oil 1 (mass \%) } & \multicolumn{5}{|c|}{ He=p OAl 2 (mass \%) } & \multicolumn{5}{|c|}{ Help or 2a (mass \%) } \\
\hline & & $\mathbf{A}$ & $\mathbf{B}$ & $\mathbf{C}$ & Avg & SD & $\bar{A}$ & $\overline{\mathbf{B}}$ & $\mathbf{C}$ & Avg & SD & $\bar{A}$ & $\mathbf{B}$ & $\mathbf{C}$ & Avg & SD \\
\hline \multirow{41}{*}{ 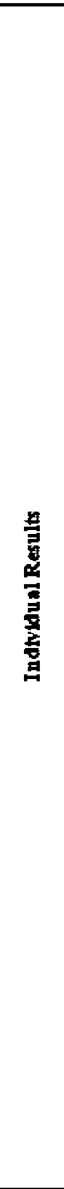 } & NIST & & & & 0.039 & 0.010 & & & & 0.142 & 0.016 & & & & 0.127 & 0.016 \\
\hline & $\mathrm{A} 002$ & 0.04011 & 0.03892 & 0.02849 & 0.0358 & 0.0064 & 0.14816 & 0.140093 & 0.148368 & 0.1455 & 0.0047 & & & & & \\
\hline & A 003 & & & & & & 0247 & 0248 & 0.237 & 0.2440 & 0.0061 & & & & & \\
\hline & A 005 & 0.0517 & 0.0469 & 0.0533 & 0.0506 & 0.0033 & 0.168 & 0.167 & 0.168 & 0.1677 & 0.0006 & & & & & \\
\hline & A006 & 0.13 & & & 0.1300 & & 0.26 & & & 0.26000 & & & & & & \\
\hline & A 007 & & & & & & & 0.02 & & 0.0200 & & & & & & \\
\hline & A 008 & 0.133 & & & 01330 & & & & & & & & & & & \\
\hline & $\mathrm{A} 009$ & & & & & & & & & & & 0.132 & 0.149 & 0.132 & 0.1377 & 0.0098 \\
\hline & A 010 & & & & & & & & & & & present & pressent & present & & \\
\hline & $\mathrm{A} 012$ & & & & & & 0.14966 & 0.15056 & 0.14932 & 0.1498 & 0.0006 & & & & & \\
\hline & A013 & 0.058 & 0.047 & 0.049 & 0.0513 & 0.0059 & $0 \_161$ & 0.177 & 0.177 & 0.1717 & 0.0092 & & & & & \\
\hline & A 014 & $<0.09$ & & & $<0.09$ & & 0.14 & & & 0.1400 & & & & & & \\
\hline & A015 & & & & & & & & & & & 0.15 & 0.14 & 0.16 & 0.1500 & 0.0100 \\
\hline & A 016 & & & & & & & & & & & & & & & \\
\hline & A017 & 0.05 & & & 0.0500 & & 0.17 & & & 0.1700 & & & & & & \\
\hline & $\mathrm{A} 018$ & & & & & & & & & & & & & & & \\
\hline & A019 & $<0.09$ & & & $<0.09$ & & 0.14 & & & 0.1400 & & & & & & \\
\hline & $\mathrm{A} 020$ & 0.0322 & 0.032346 & 0.031258 & 0.0319 & 0.0006 & 0.131187 & 0.130074 & 0.13407 & 0.1318 & 0.0021 & & & & & \\
\hline & A021 & & & & & & & & & & & 0.12 & 0.12 & 0.13 & 0.1233 & 0.0058 \\
\hline & $\mathrm{A} 022$ & & & & & & & & & & & 0.0876 & 0.0876 & 0.088 & 0.0877 & 0.0002 \\
\hline & A 023 & & & & & & 0.2095 & 0239 & 0.2213 & 0.2233 & $0 \_0148$ & & & & & \\
\hline & A 024 & & & & & & & & & & & 0.142 & 0.142 & 0.172 & 0.1520 & 0.0173 \\
\hline & A025 & & & & & & & & & & & & & & & \\
\hline & $\mathrm{A} 027$ & & & & & & & & & & & 0.143 & 0.144 & 0.144 & 0.1437 & 0.0006 \\
\hline & A031 & & & & & & & & & & & & & & & \\
\hline & A 033 & 0.05 & 0.04 & 0.04 & 0.0433 & 0.0058 & 0.15 & 0.14 & 0.15 & 0.1467 & 0.0058 & & & & & \\
\hline & A035 & 0.0502 & & & 0.0502 & & 0.152 & 0.152 & 0.152 & 0.1520 & 0.0000 & & & & & \\
\hline & A 036 & $<0.206$ & $<0.206$ & $<0.206$ & $<0.206$ & & 0.121 & 0.121 & 0.124 & 0.1220 & 0.0017 & & & & & \\
\hline & A038 & 0.0462 & 0.0483 & 0.0479 & 0.0475 & 0.0011 & 0.161 & 0.159 & 0.155 & 0.1583 & 0.0031 & & & & & \\
\hline & A 039 & 0.03 & 0.03 & 0.03 & 0.0300 & 0.0000 & 0.11 & 0.12 & 0.12 & 0.1167 & 0.0058 & & & & & \\
\hline & A040 & & & & & & & & & & & $0 \_14257$ & 0.13653 & 0.14842 & 0.1425 & 0.0059 \\
\hline & A 041 & 0 & 0 & 0 & 0.0000 & 0.0000 & 0.1 & 0.1 & 0.1 & 0.1000 & 0.0000 & & & & & \\
\hline & A043 & 0.08 & 0.079 & 0.081 & 0.0800 & $0 \_0010$ & 0.203 & 02207 & 0.207 & 0.2057 & 0.0023 & & & & & \\
\hline & A 044 & & & & & & & & & & & & & & & \\
\hline & A045 & & & & & & & & & & & & & & & \\
\hline & A 046 & 0 & 0 & 0 & 0.0000 & 0.0000 & 0 & 0.14 & 0.13 & 0.0900 & 0.0781 & & & & & \\
\hline & A050 & $<0.01$ & $<0.01$ & $<0.01$ & $<0.01$ & & 0.152 & 0.183 & 0.152 & 0.1623 & $0 \_0179$ & & & & & \\
\hline & A 052 & & & & & & & & & & & & & & & \\
\hline & A053 & & & & & & & & & & & & & & & \\
\hline & $\mathrm{A} 054$ & $<0.07$ & $<0.07$ & $<0.07$ & $<0.07$ & & 0.15 & 0.13 & 0.13 & 0.1367 & 0.0115 & & & & & \\
\hline & A055 & 0.0502 & 0.0518 & 0.0441 & 0.0487 & 0.0041 & 0.15 & 0.1523 & 0.1426 & 0.1483 & 0.0051 & & & & & \\
\hline 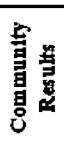 & & $\begin{array}{l}\text { Consensu } \\
\text { Consensu } \\
\text { Maximum } \\
\text { Minimum } \\
\mathrm{N}\end{array}$ & $\begin{array}{l}\text { ss Mean } \\
\text { s Standard I } \\
\text { a }\end{array}$ & Deviation & $\begin{array}{c}0.0393 \\
0.0034 \\
0.1330 \\
0.0000 \\
30\end{array}$ & & $\begin{array}{l}\text { Consensu } \\
\text { Consensu } \\
\text { Maximum } \\
\text { Minimum } \\
\text { N }\end{array}$ & $\begin{array}{l}\text { s Mean } \\
\text { s Standard I }\end{array}$ & Deviation & $\begin{array}{c}0.1421 \\
0.0046 \\
0.3067 \\
0.0037 \\
42\end{array}$ & & $\begin{array}{l}\text { Consensu: } \\
\text { Consensu: } \\
\text { Maximum } \\
\text { Minimum } \\
\text { N }\end{array}$ & $\begin{array}{l}\text { Mean } \\
\text { Stmdand }\end{array}$ & Deviation & $\begin{array}{c}0.1328 \\
0.0084 \\
0.2000 \\
0.0877 \\
10\end{array}$ & \\
\hline
\end{tabular}




\begin{tabular}{|c|c|c|c|c|c|c|c|c|c|c|c|c|c|c|c|c|c|}
\hline & & & & & & & & & Cannat & bidivarin ( & EDV) & & & & & & \\
\hline & & & & Heap & OAl 1 (n) & $5 \%)$ & & & Henp & Oll $2(\mathrm{mx}$ & (s \%) & & & He= & 2) & ss \%) & \\
\hline & & Lab & $\mathbf{A}$ & B & $\mathbf{C}$ & Avg & SD & $\mathbf{A}$ & B & $\mathbf{C}$ & Avg & SD & $\mathbf{A}$ & B & $\mathbf{C}$ & Avg & SD \\
\hline & & NIST & & & & 0.039 & 0.010 & & & & 0.142 & 0.016 & & & & 0.127 & 0.016 \\
\hline & & A 057 & & & & & & & & & & & & & & & \\
\hline & & A 060 & 0.04 & 0.04 & 0.04 & 0.0400 & 0.0000 & 0.14 & 0.15 & 0.15 & 0.1467 & 0.0058 & & & & & \\
\hline & & A 061 & 0.031 & 0.032 & 0.031 & 0.0313 & 0.0006 & 0.133 & 0.129 & 0.132 & 0.1313 & 0.0021 & & & & & \\
\hline- & & A 062 & & & & & & & & & & & 0.1 & 0.1 & 0.103 & 0.1010 & $0 \_0017$ \\
\hline च & & A 063 & & & & & & 0.11679 & 0.11646 & 0.12307 & 0.1188 & 0.0037 & & & & & \\
\hline$\vec{\sim}$. & & A 064 & & & & & & & & & & & & & & & \\
\hline$\nabla$ & & A 066 & yes & yes & yes & & & & & & & & & & & & \\
\hline$\Xi$ & & A068 & & & & & & & & & & & & & & & \\
\hline$\sigma$ & & A 071 & 0.03 & & & 0.0300 & & & & & & & & & & & \\
\hline$\vec{\Omega}$ & & A072 & & & & & & & & & & & & & & & \\
\hline$\stackrel{0}{\Rightarrow}$ & & A 073 & 0.047 & 0.045 & 0.044 & 0.0453 & 0.0015 & 0.141 & 0.14 & 0.142 & 0.1410 & 0.0010 & & & & & \\
\hline 0 & & A074 & 0.034 & $0 \_033$ & $0 \_034$ & 0.0337 & 0.0006 & 0.134 & 0.137 & 0.133 & 0.1347 & 0.0021 & & & & & \\
\hline 戸 & & A 076 & 0.00115 & 0.00117 & 0.001162 & 0.0012 & 0.0000 & 0.003672 & 0.003681 & 0.003669 & 0.0037 & 0.0000 & & & & & \\
\hline$\vec{\sim}$. & & A 077 & & & & & & $\angle \mathrm{TOQ}$ & $\angle \mathrm{LOQ}$ & $\angle I O Q$ & & & & & & & \\
\hline טم & & A 081 & 0.033 & 0.033 & 0.033 & 0.0330 & 0.0000 & 0.112 & 0.123 & 0.112 & 0.1157 & 0.0064 & & & & & \\
\hline$<$ & & A082 & & & & & & & & & & & 0.2 & & & 0.2000 & \\
\hline ص. & & A 083 & 0.07 & & & 0.0700 & & 0.17 & & & 0.1700 & & & & & & \\
\hline$\tilde{D}$ & & A 084 & 0.046 & 0.046 & 0.044 & 0.0453 & 0.0012 & 0.159 & 0.159 & 0.159 & 0.1590 & 0.0000 & & & & & \\
\hline$\underline{\sigma}$ & 告 & A 085 & 0.04 & 0.04 & 0.04 & 0.0400 & 0.0000 & 0.15 & 0.16 & 0.15 & 0.1533 & 0.0058 & & & & & \\
\hline $\bar{D}$ & 尼 & A087 & & & & & & & & & & & & & & & \\
\hline 豆 & 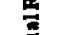 & A 088 & & & & & & 0.14 & 0.15 & 0.14 & 0.1433 & 0.0058 & & & & & \\
\hline 8 & 寻 & A 089 & 0.07 & 0.07 & 0.04 & 0.0600 & 0.0173 & 0.14 & 0.12 & 0.11 & 0.1233 & 0.0153 & & & & & \\
\hline 0 & $\frac{\pi}{8}$ & A 090 & & & & & & & & & & & 0.1175 & 0.1151 & 0.113 & 0.1152 & 0.0023 \\
\hline$\stackrel{0}{\rightarrow}$ & 总 & A091 & & & & & & & & & & & & & & & \\
\hline$\rho$ & & A 092 & 0.0373 & 0.0371 & 0.0376 & 0.0373 & 0.0003 & 0.1434 & 0.1426 & 0.1438 & 0.1433 & 0.0006 & & & & & \\
\hline$\widetilde{0}$ & & A093 & & & & & & & & & & & & & & & \\
\hline 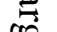 & & A 095 & 0.0584 & 0.0565 & 0.0575 & 0.0575 & 0.0010 & 0.1445 & 0.1446 & 0.1442 & 0.1444 & 0.0002 & & & & & \\
\hline (1) & & A096 & & & & & & & & & & & & & & & \\
\hline$\exists$ & & A 098 & 0.075 & & & 0.0750 & & 0.17 & & & 0.1700 & & & & & & \\
\hline $\overrightarrow{0}^{\prime}$ & & A099 & 0.033 & 0.033 & 0.033 & 0.0330 & 0.0000 & 0.135 & 0.135 & 0.134 & 0.1347 & 00006 & & & & & \\
\hline 互 & & A 100 & $<0.0210$ & $<0.0210$ & 0.02118 & 0.0212 & & 0.1131 & 0.1093 & 0.1123 & 0.1116 & 0.0020 & & & & & \\
\hline 5 & & A 101 & & & & & & & & & & & & & & & \\
\hline$\Xi$ & & A 102 & 0.02 & 0.02 & 0.02 & 0.0200 & 0.0000 & 0.11 & 0.11 & 0.11 & 0.1100 & 0.0000 & & & & & \\
\hline 훙 & & A 103 & 0 & 0 & 0 & 00000 & 0.0000 & 0.076 & 0.098 & 0.118 & 0.0973 & 0.0210 & & & & & \\
\hline • & & A 104 & $<0.037$ & $<0.033$ & $<0.042$ & & & 0.227 & 0.253 & 0.274 & 0.2513 & 0.0235 & & & & & \\
\hline$\vdots$ & & A 107 & 0.08 & 0.0759 & 0.0787 & 0.0782 & 0.0021 & 0.185 & 0.181 & 0.0165 & 0.1275 & 0.0961 & & & & & \\
\hline 른 & & A 108 & & & & & & & & & & & & & & & \\
\hline$\stackrel{0}{ }$ & & A 109 & 0.04 & & & 0.0400 & & 0.14 & 0.12 & & 0.1300 & 0.0141 & & & & & \\
\hline 0 & & A 110 & 0.12 & 0.12 & 0.12 & 0.1200 & 0.0000 & 0.29 & 0.3 & 0.33 & 0.3067 & 0.0208 & & & & & \\
\hline $\overrightarrow{00}$ & & A112 & 00267 & 0.0267 & 0.0267 & 0.0267 & 0.0000 & 0.112 & 0.13 & 0.121 & 0.1210 & 00090 & & & & & \\
\hline$\Xi$ & & A 113 & 0.029962 & 0.030023 & 0.03 & 0.0300 & 0.0000 & 0.13993 & 0.139091 & 0.138112 & 0.1390 & 0.0009 & & & & & \\
\hline$c$ & & A115 & & & & & & & & & & & 0.13 & 0.13 & 0.13 & 0.1300 & 0.0000 \\
\hline a & & A 116 & 0.005 & 0.028 & 0.0268 & 0.0199 & 0.0129 & 0.108 & 0.15 & 0.1341 & 0.1307 & 0.0212 & & & & & \\
\hline $\begin{array}{l}\infty \\
\infty \\
\infty \\
\infty\end{array}$ & 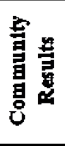 & & \begin{tabular}{|l} 
Consensus \\
Consensus \\
Maximum \\
Minimum \\
$\mathrm{N}$ \\
\end{tabular} & $\begin{array}{l}\text { s Mean } \\
\text { s Standand }\end{array}$ & Deviation & $\begin{array}{c}0.0393 \\
0.0034 \\
0.1330 \\
0.0000 \\
30 \\
\end{array}$ & & $\begin{array}{l}\text { Consensus } \\
\text { Consensu: } \\
\text { Maximum } \\
\text { Minimum } \\
\mathrm{N}\end{array}$ & $\begin{array}{l}\text { s Mean } \\
\text { s Standard }\end{array}$ & Deviation & $\begin{array}{c}0.1421 \\
0.0046 \\
0.3067 \\
0.0037 \\
42 \\
\end{array}$ & & \begin{tabular}{|l|} 
Consens \\
Consens \\
Maximur \\
Minimum \\
N \\
\end{tabular} & $\begin{array}{l}\text { Mean } \\
\text { Standare }\end{array}$ & eviation & $\begin{array}{c}0.1328 \\
0.0084 \\
0.2000 \\
0.0877 \\
10 \\
\end{array}$ & \\
\hline
\end{tabular}




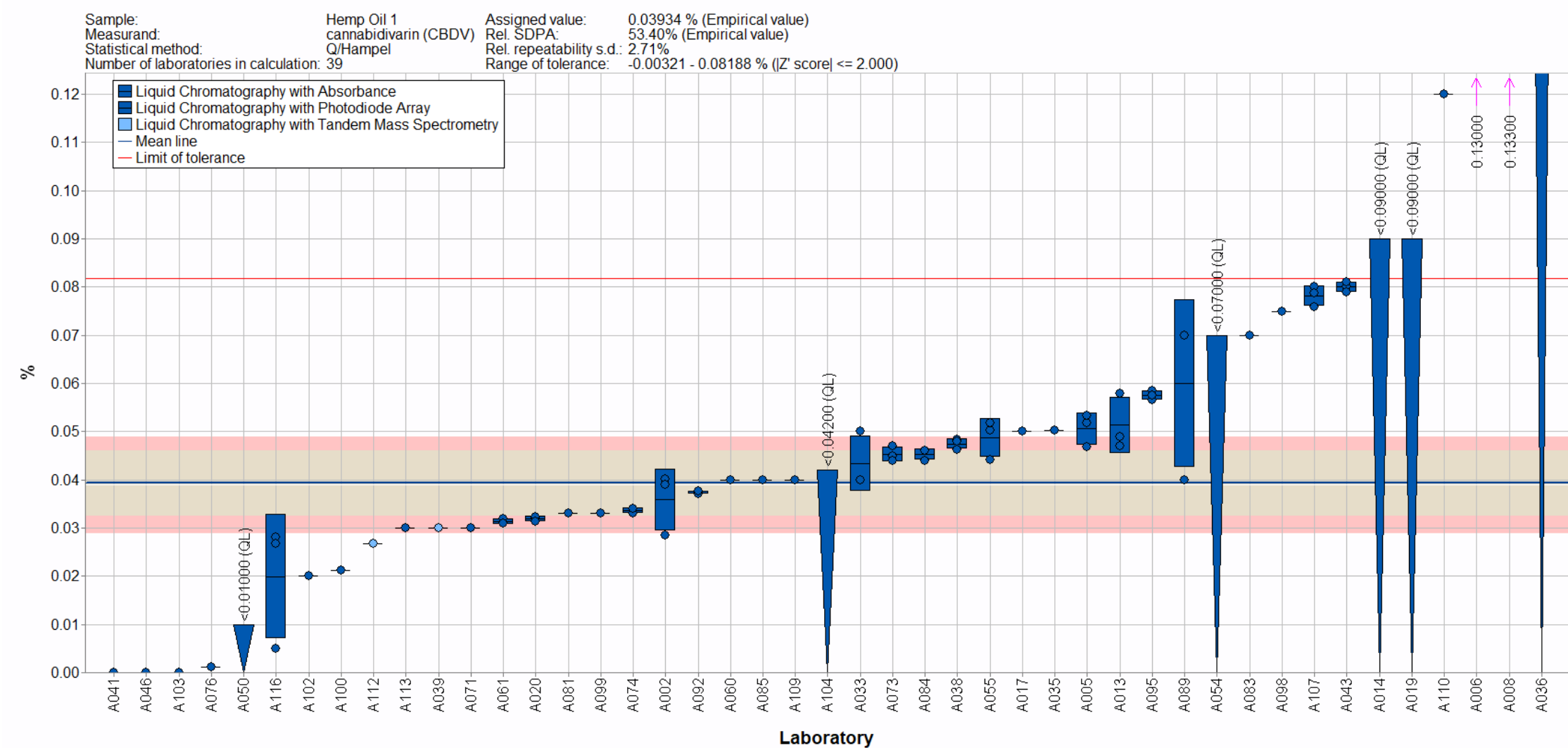

Figure 5-1. CBDV in Hemp Oil 1 (data summary view - analytical method). In this view, individual laboratory data are plotted (circles) with the individual laboratory standard deviation (rectangle). The color of the data point represents the analytical method employed. The solid blue line represents the consensus mean, and the green shaded region represents the $95 \%$ confidence interval for the consensus mean. The solid red line represents the upper consensus range of tolerance, calculated as the values above the consensus mean that results in an acceptable $Z_{\text {comm }}^{\prime}$ score, $\left|Z_{\text {comm }}^{\prime}\right| \leq 2$, with the lower limit set at zero. The red shaded region represents the NIST range of tolerance, which encompasses the target value bounded by twice its uncertainty ( $\left.U_{\mathrm{NIST}}\right)$ and represents the range that results in an acceptable $Z_{\text {NIST }}$ score, $\left|Z_{\text {NIST }}\right| \leq 2$. The shaded beige region represents the overlapping of the $95 \%$ confidence interval for the consensus mean (green region) and the NIST range of tolerance (red region). The downward triangle represents data reported as a threshold or LOQ value. 


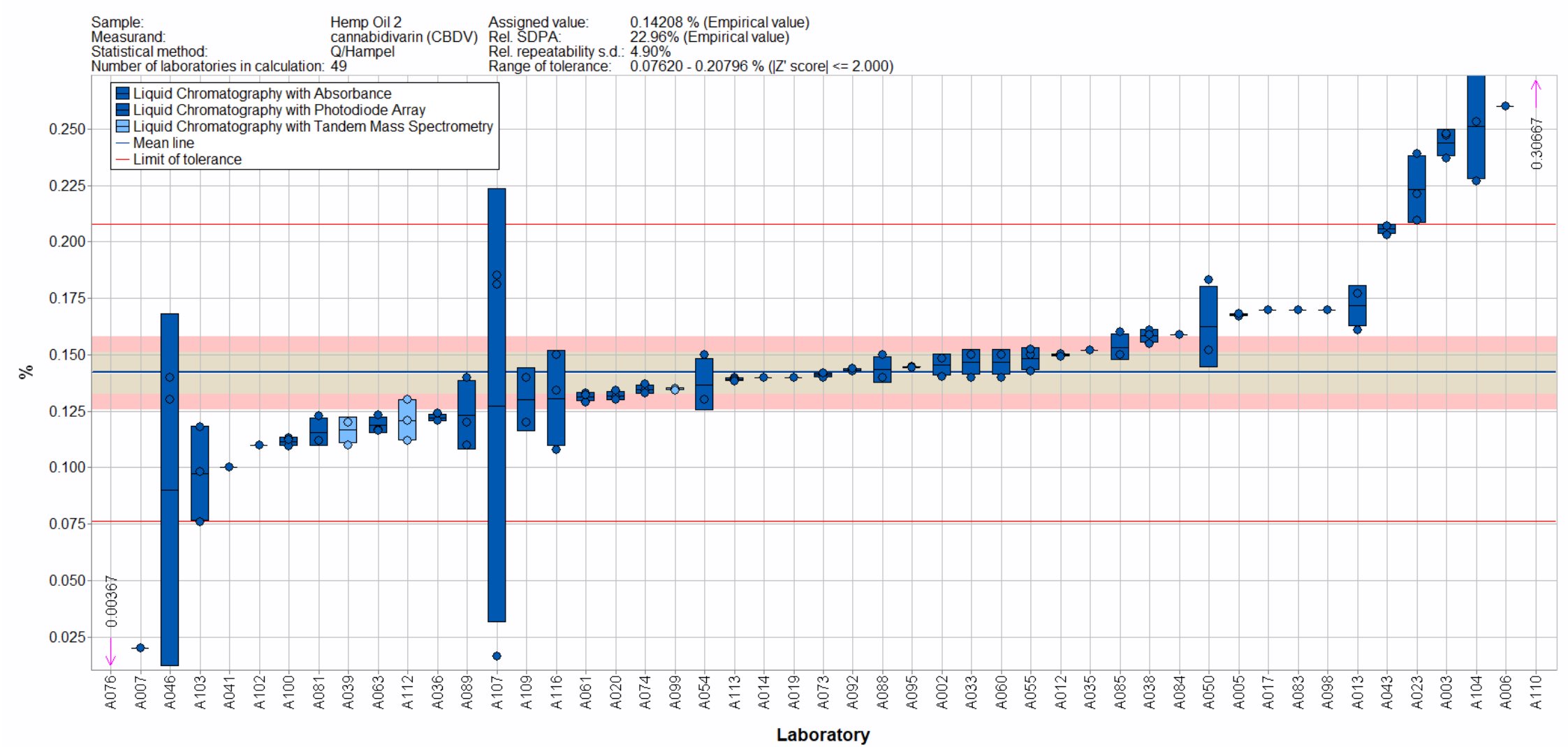

Figure 5-2. CBDV in Hemp Oil 2 (data summary view - analytical method). In this view, individual laboratory data are plotted (circles) with the individual laboratory standard deviation (rectangle). The color of the data point represents the analytical method employed. The solid blue line represents the consensus mean, and the green shaded region represents the $95 \%$ confidence interval for the consensus mean. The solid red lines represent the consensus range of tolerance, calculated as the values above and below the consensus mean that result in an acceptable $Z_{\text {comm }}^{\prime}$ score, $\left|Z_{\text {comm }}^{\prime}\right| \leq 2$. The red shaded region represents the NIST range of tolerance, which encompasses the target value bounded by twice its uncertainty ( $U_{\text {NIST }}$ ) and represents the range that results in an acceptable $Z_{\text {NIST }}$ score, $\left|Z_{\text {NIST }}\right| \leq 2$. The shaded beige region represents the overlapping of the $95 \%$ confidence interval for the consensus mean (green region) and the NIST range of tolerance (red region). 


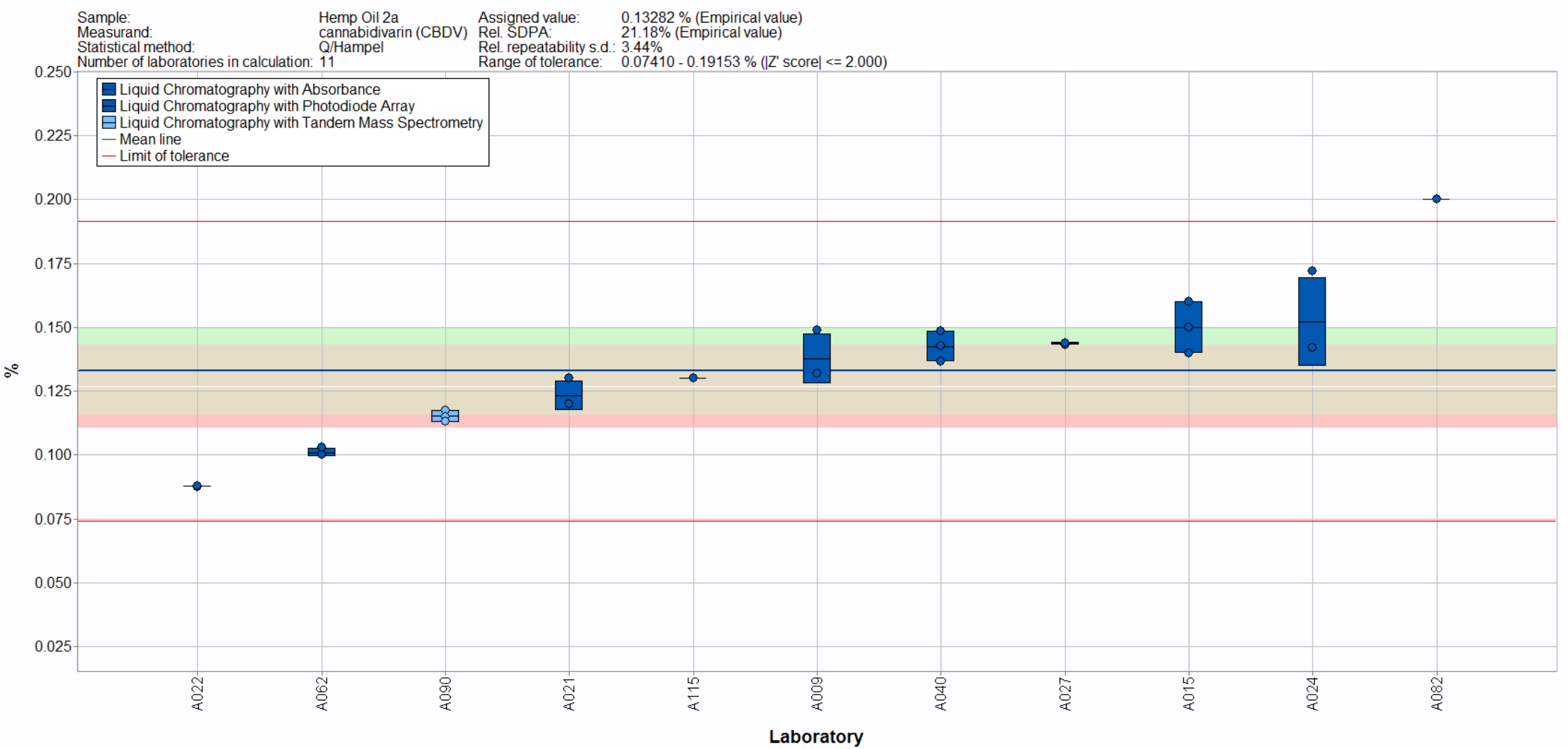

Figure 5-3. CBDV in Hemp Oil 2a (data summary view - analytical method). In this view, individual laboratory data are plotted (circles) with the individual laboratory standard deviation (rectangle). The color of the data point represents the analytical method employed. The solid blue line represents the consensus mean, and the green shaded region represents the $95 \%$ confidence interval for the consensus mean. The solid red lines represent the consensus range of tolerance, calculated as the values above and below the consensus mean that result in an acceptable $Z_{\text {comm }}^{\prime}$ score, $\left|Z_{\text {comm }}^{\prime}\right| \leq 2$. The red shaded region represents the NIST range of tolerance, which encompasses the target value bounded by twice its uncertainty ( $U_{\text {NIST }}$ ) and represents the range that results in an acceptable $Z_{\text {NIST }}$ score, $\left|Z_{\text {NIST }}\right| \leq 2$. The shaded beige region represents the overlapping of the $95 \%$ confidence interval for the consensus mean (green region) and the NIST range of tolerance (red region). 


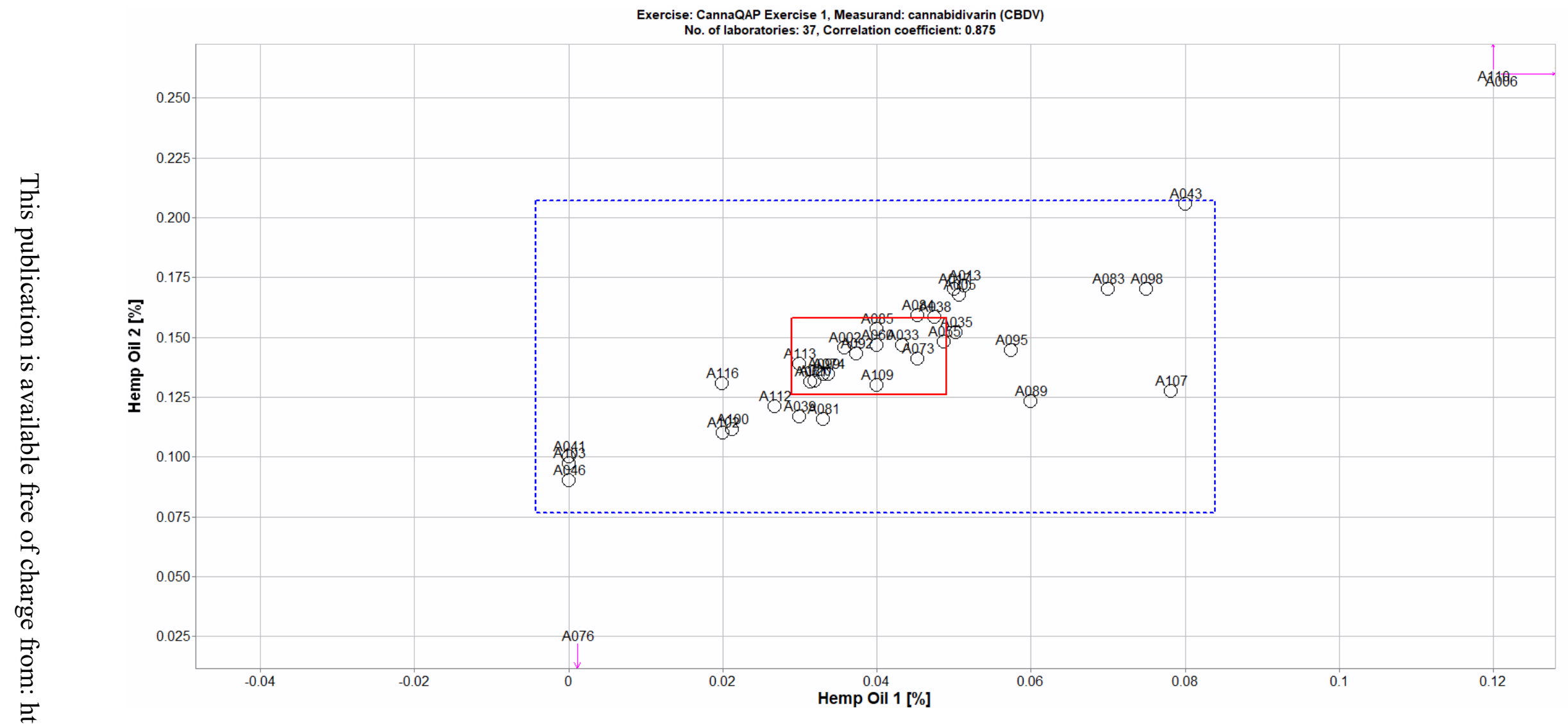

Figure 5-4. Laboratory means for CBDV in Hemp Oil 1 and Hemp Oil 2 (sample/sample comparison view). In this view, the individual laboratory mean for one sample (Hemp Oil 1) is compared to the individual laboratory mean for a second sample (Hemp Oil 2). The solid red box represents the NIST range of tolerance for the two samples, Hemp Oil 1 (x-axis) and Hemp Oil 2 (y-axis), which encompasses the target values bounded by their uncertainties ( $\left.U_{\text {NIST }}\right)$ and represents the range that results in an acceptable $Z_{\text {NIST }}$ score, $\left|Z_{\text {NIST }}\right| \leq 2$. The dotted blue box represents the consensus range of tolerance for Hemp Oil 1 (x-axis) and Hemp Oil 2 (y-axis), calculated as the values above and below the consensus means that result in an acceptable $Z_{\text {comm }}^{\prime}$ score, $\left|Z_{\text {comm }}^{\prime}\right| \leq 2$. 
Table 5-3. Data summary table for CBDVA in hemp oils. Data highlighted in red have been flagged as a data entry of zero or results that include text (e.g., "< LOQ" or "present"). Data highlighted in blue have been identified as outside the consensus tolerance limits and would be estimated to result in an unacceptable $Z_{\text {comm }}^{\prime}$ score, $\left|Z_{\text {comm }}^{\prime}\right| \geq 2$.

\begin{tabular}{|c|c|c|c|c|c|c|c|c|c|c|c|c|c|c|c|c|}
\hline & \multirow[b]{3}{*}{ Lab } & \\
\hline & & \multicolumn{15}{|c|}{$\begin{array}{c}\text { Cannabidirarinic adid (CBDVA) } \\
\text { Hewp Oil2 (-ass \%) }\end{array}$} \\
\hline & & $\mathbf{A}$ & B & $\mathbf{C}$ & Avg & SD & $\mathbf{A}$ & B & $\mathbf{c}$ & Avg & SD & $\mathbf{A}$ & B & $\mathbf{C}$ & Avg & SD \\
\hline \multirow{60}{*}{ 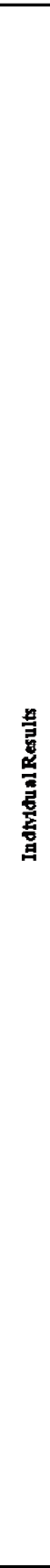 } & NIST & & & & & & & & & & & & & & & \\
\hline & $\mathrm{A} 002$ & $<0.0021$ & $<0.0021$ & $<0.0021$ & $<0.0021$ & & $<0.0021$ & $<0.0021$ & $<0.0021$ & $<0.0021$ & & & & & & \\
\hline & A005 & 0 & 0 & 0 & 00000 & 00000 & 0 & 0 & 0 & 0.0000 & 0.0000 & & & & & \\
\hline & A 009 & & & & & & & & & & & $<0.00100$ & $<0.00100$ & $<0.00100$ & $<0.00100$ & \\
\hline & A010 & & & & & & & & & & & & & & & \\
\hline & A 014 & $<0.09$ & & & $<0.09$ & & $<0.09$ & & & & & & & & & \\
\hline & A015 & & & & & & & & & & & & & & & \\
\hline & A 016 & & & & & & & & & & & & & & & \\
\hline & A017 & $<0.01$ & & & 40.01 & & 0.01 & & & 0.0100 & & & & & & \\
\hline & A 018 & & & & & & & & & & & & & & & \\
\hline & A019 & $<009$ & & & 409 & & $<0.09$ & & & & & & & & & \\
\hline & $\mathrm{A} 020$ & & & & & & & & & & & & & & & \\
\hline & A021 & & & & & & & & & & & $<0.05$ & $<0.05$ & $<0.05$ & $<0.05$ & \\
\hline & $\mathrm{A} 022$ & & & & & & & & & & & & & & & \\
\hline & $\mathrm{A} 023$ & & & & & & 0 & 0 & 0 & 0.0000 & 0.0000 & & & & & \\
\hline & A 024 & & & & & & & & & & & & & & & \\
\hline & A025 & & & & & & 004 & 0.04 & 0.04 & 00400 & 0.0000 & & & & & \\
\hline & A 027 & & & & & & & & & & & $<0.0057$ & $<0.0057$ & $<0.0057$ & $\mid<0.0057$ & \\
\hline & A031 & & & & & & & & & & & & & & & \\
\hline & A 035 & $<0.0025$ & & & $<0.0025$ & & $<0.0025$ & $<0.0025$ & $<0.0025$ & $<0.0025$ & & & & & & \\
\hline & A038 & $<0025$ & $<0.025$ & $<0.025$ & $<0.025$ & & $<0025$ & $<0.025$ & $<0.025$ & $<0.025$ & & & & & & \\
\hline & A 040 & & & & & & & & & & & & & & & \\
\hline & A041 & 0 & 0 & 0 & 00000 & 00000 & 0 & 0 & 0 & 0.0000 & 0.0000 & & & & & \\
\hline & A 043 & 0.004 & 0.005 & 0.005 & 0.0047 & 0.0006 & 0.006 & 0.005 & 0.005 & 0.0053 & 0.0006 & & & & & \\
\hline & A044 & & & & & & & & & & & & & & & \\
\hline & A 045 & & & & & & & & & & & & & & & \\
\hline & A 046 & 0 & 0 & 0 & 00000 & 00000 & 0 & 0 & 0 & 0.0000 & 0.0000 & & & & & \\
\hline & $\mathrm{A} 052$ & & & & & & & & & & & & & & & \\
\hline & A053 & & & & & & & & & & & & & & & \\
\hline & A 055 & 0 & 0 & 0 & 0.0000 & 0.0000 & 0 & 0 & 0 & 0.0000 & 0.0000 & & & & & \\
\hline & A057 & & & & & & & & & & & & & & & \\
\hline & A 062 & & & & & & & & & & & & & & & \\
\hline & A066 & & & & & & & & & & & & & & & \\
\hline & A 068 & & & & & & & & & & & & & & & \\
\hline & A071 & & & & & & & & & & & & & & & \\
\hline & $\mathrm{A} 072$ & & & & & & & & & & & & & & & \\
\hline & $\mathrm{A} 073$ & 0 & 0 & 0 & 00000 & 0.0000 & 0 & 0 & 0 & 0.0000 & 0.0000 & & & & & \\
\hline & A 074 & $<0.007$ & $<0.007$ & $<0.007$ & $<0.007$ & & $<0.007$ & $<0.007$ & $<0.007$ & $<0.007$ & & & & & & \\
\hline & A076 & & & & & & & & & & & & & & & \\
\hline & A 082 & & & & & & & & & & & & & & & \\
\hline & A083 & $<0.33$ & & & 433 & & $<0.33$ & & & $<033$ & & & & & & \\
\hline & A 084 & 0.017 & 0.013 & 0.013 & 0.0143 & 0.0023 & $\mid<0.0125$ & $<0.0125$ & $<0.0125$ & $<0.0125$ & & & & & & \\
\hline & A087 & & & & & & & & & & & & & & & \\
\hline & A 089 & 0.03 & 0.05 & 0.01 & 0.0300 & 0.0200 & 0.02 & 0.02 & & 0.0200 & 0.0000 & & & & & \\
\hline & A090 & & & & & & & & & & & 0.0003 & 0.0003 & 0.0003 & 0 & 0 \\
\hline & $\mathrm{A} 092$ & & & & & & & & & & & & & & & \\
\hline & A093 & & & & & & & & & & & & & & & \\
\hline & A 095 & $<0.0300$ & $<0.0300$ & $<0.0300$ & $<0.0300$ & & $\mid<0.0300$ & $<0.0300$ & $<0.0300$ & $<0.0300$ & & & & & & \\
\hline & A096 & & & & & & & & & & & & & & & \\
\hline & A 098 & $<0.0046$ & & & $<0.0046$ & & $\mid<0.0025$ & & & $<0.0025$ & & & & & & \\
\hline & A099 & $<0.007$ & $<0.007$ & $<0.007$ & $<0.007$ & & 0.021 & 0.021 & 0.021 & 0.0210 & 0.0000 & & & & & \\
\hline & A 100 & $<0.0210$ & $<0.0210$ & $<0.0210$ & $<0.0210$ & & 0.04424 & 0.04328 & 0.04347 & 0.0437 & 0.0005 & & & & & \\
\hline & A 101 & & & & & & & & & & & & & & & \\
\hline & A 102 & $<0.01$ & $<0.01$ & $<0.01$ & $<0.01$ & & $<0.01$ & $<0.01$ & $<0.01$ & $<0.01$ & & & & & & \\
\hline & A 104 & $<0.037$ & $<0.033$ & $<0.042$ & $<0.037$ & & $<0.033$ & $<0.026$ & $<0.034$ & $<0.033$ & & & & & & \\
\hline & A 107 & 0.0059 & 0.00571 & $<0.01$ & 0.0058 & 0.0001 & 0.0231 & 0.0226 & 0.0215 & 0.0224 & 0.0008 & & & & & \\
\hline & A 108 & & & & & & & & & & & & & & & \\
\hline & A 112 & & & & & & & & & & & & & & & \\
\hline & A113 & & & & & & & & & & & & & & & \\
\hline & A 115 & & & & & & & & & & & & & & & \\
\hline 賈臂 & & $\begin{array}{l}\text { Consensus } \\
\text { Consensus } \\
\text { Maximum } \\
\text { Minimum } \\
\end{array}$ & $\begin{array}{l}\text { s Mean } \\
\text { s Standard I } \\
\text { I }\end{array}$ & Deviation & $\begin{array}{c}00027 \\
0.0019 \\
0.0300 \\
0.0000 \\
9\end{array}$ & & \begin{tabular}{|l|} 
Consensu \\
Consensu \\
Maximun \\
Mimimum \\
\end{tabular} & $\begin{array}{l}\text { is Mean } \\
\text { is Standand I } \\
\text { I }\end{array}$ & Deviation & $\begin{array}{c}0.0109 \\
0.0038 \\
0.0437 \\
0.0000 \\
12\end{array}$ & & \begin{tabular}{|l} 
Consensus \\
Consensus \\
Maximum \\
Minimum \\
\end{tabular} & $\begin{array}{l}\text { s Mean } \\
\text { s Standand I } \\
\text { I }\end{array}$ & Deviation & $\begin{array}{l}0.0003 \\
0.0003\end{array}$ & \\
\hline
\end{tabular}




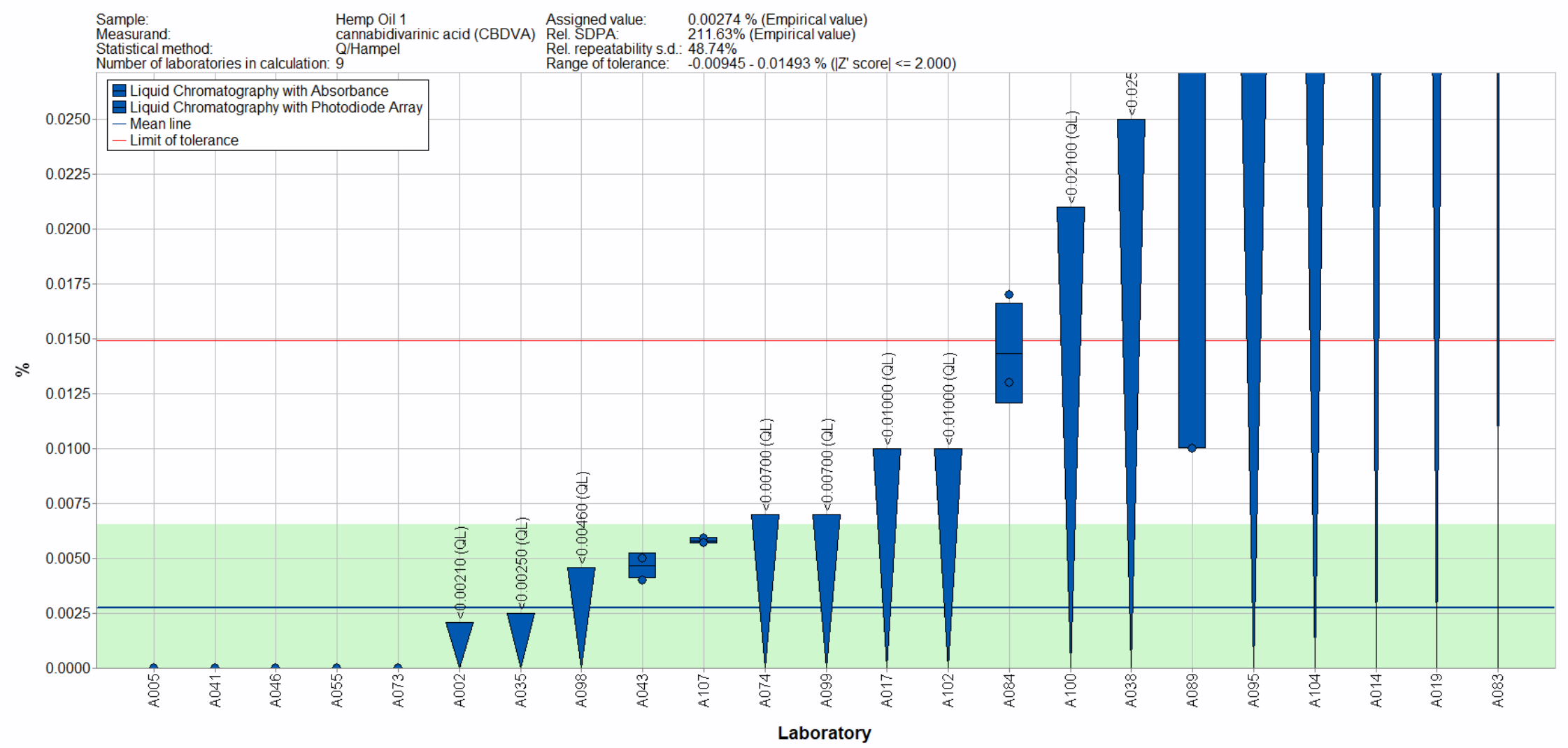

Figure 5-5. CBDVA in Hemp Oil 1 (data summary view - analytical method). In this view, individual laboratory data are plotted (circles) with the individual laboratory standard deviation (rectangle). The color of the data point represents the analytical method employed. The solid blue line represents the consensus mean, and the green shaded region represents the $95 \%$ confidence interval for the consensus mean. The solid red line represents the upper consensus range of tolerance, calculated as the values above the consensus mean that results in an acceptable $Z_{\text {comm }}^{\prime}$ score, $\left|Z_{\text {comm }}^{\prime}\right| \leq 2$, with the lower limit set at zero. A NIST value has not been determined in this material. The downward triangle represents data reported as a threshold or LOQ value. 


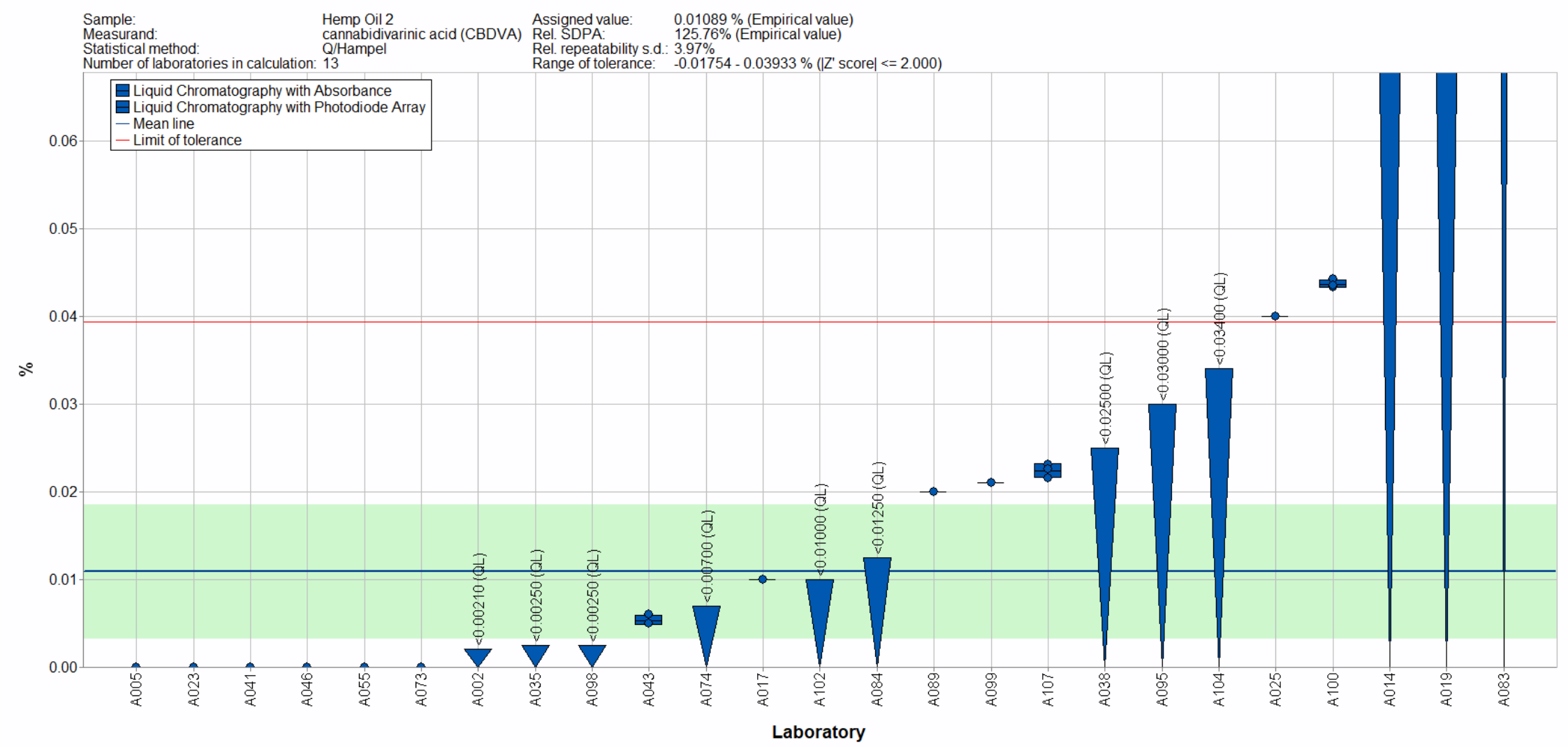

Figure 5-6. CBDVA in Hemp Oil 2 (data summary view - analytical method). In this view, individual laboratory data are plotted (circles) with the individual laboratory standard deviation (rectangle). The color of the data point represents the analytical method employed. The solid blue line represents the consensus mean, and the green shaded region represents the $95 \%$ confidence interval for the consensus mean. The solid red line represents the upper consensus range of tolerance, calculated as the values above the consensus mean that results in an acceptable $Z_{\text {comm }}^{\prime}$ score, $\left|Z_{\text {comm }}^{\prime}\right| \leq 2$, with the lower limit set at zero. A NIST value has not been determined in this material. The downward triangle represents data reported as a threshold or LOQ value. 


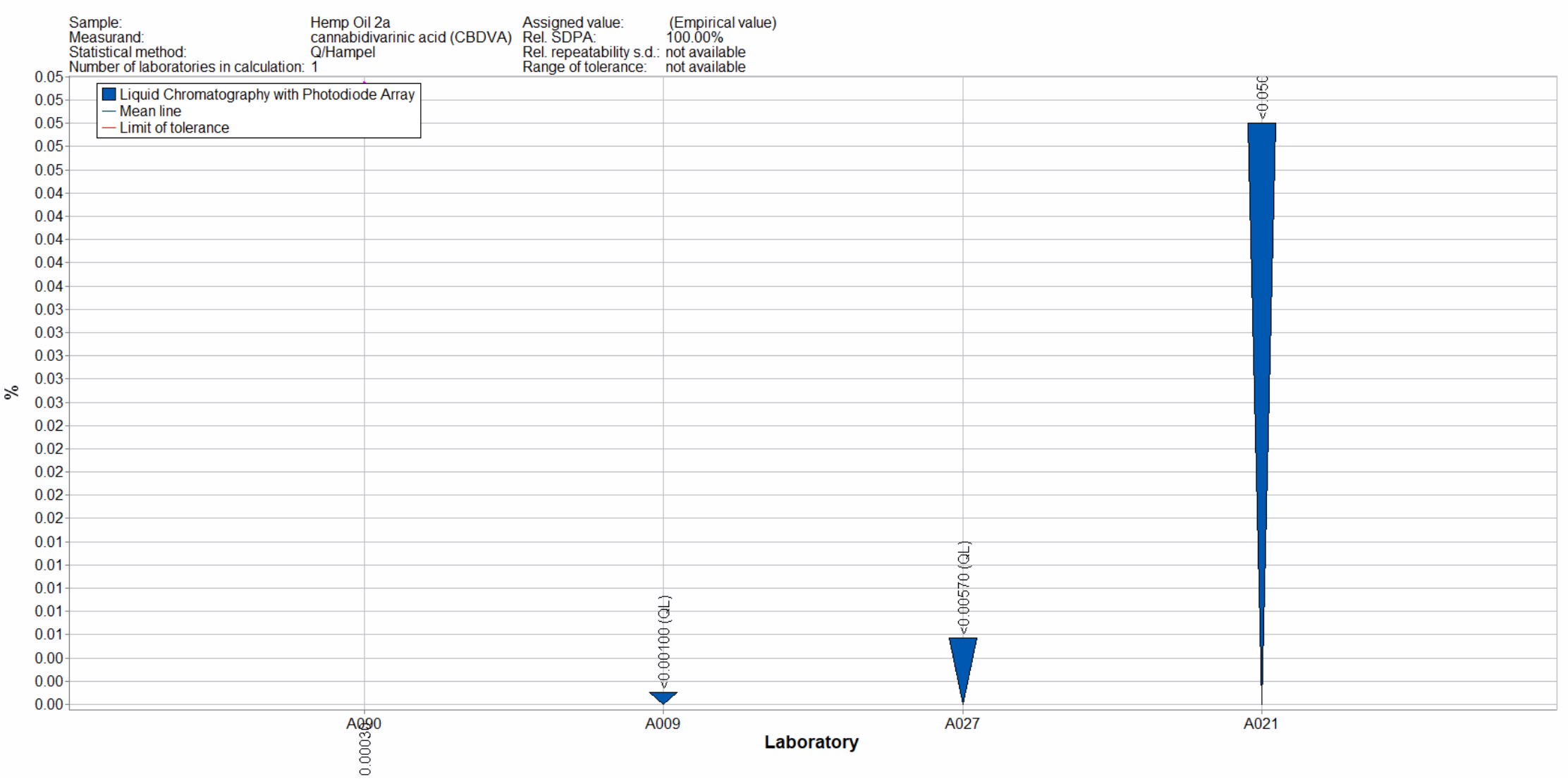

Figure 5-7. CBDVA in Hemp Oil 2a (data summary view - analytical method). In this view, individual laboratory data are plotted (circles) with the individual laboratory standard deviation (rectangle). The color of the data point represents the analytical method employed. The downward triangle represents data reported as a threshold or LOQ value. 


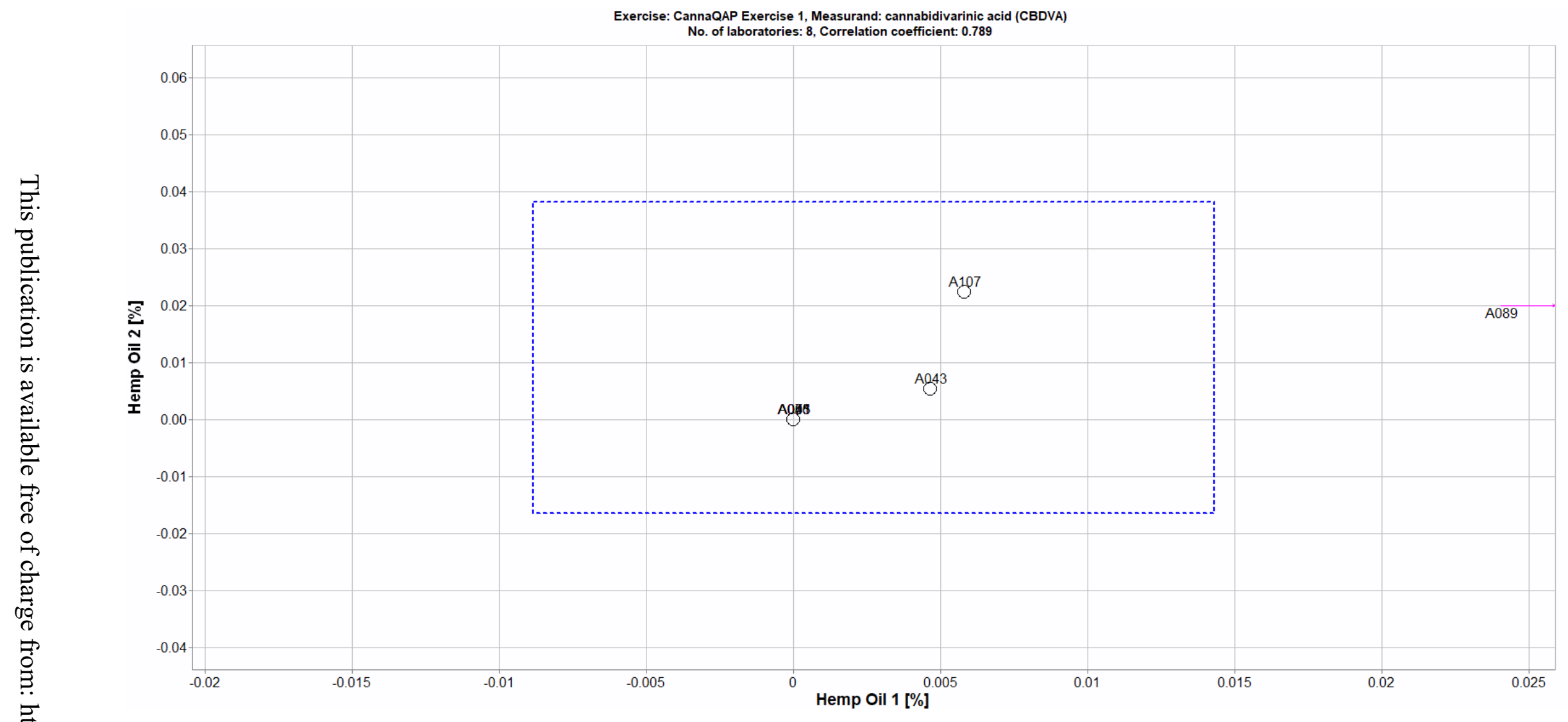

Figure 5-8. Laboratory means for CBDVA in Hemp Oil 1 and Hemp Oil 2 (sample/sample comparison view). In this view, the individual laboratory mean for one sample (Hemp Oil 1) is compared to the individual laboratory mean for a second sample (Hemp Oil 2). The dotted blue box represents the consensus range of tolerance for Hemp Oil 1 (x-axis) and Hemp Oil 2 (y-axis), calculated as the values above and below the consensus means that result in an acceptable $Z_{\text {comm }}^{\prime}$ score, $\left|Z_{\text {comm }}^{\prime}\right| \leq 2$. 


\section{SECTION 6: CBG AND CBGA}

Study Overview

CBG is a non-intoxicating cannabinoid often detected in Cannabis plants and Cannabis-derived products. CBG has attracted significant research interest and reliable analytical methods are necessary to explore the potential health benefits. ${ }^{9} \mathrm{CBG}$ does not exist in Cannabis plant naturally but is formed through decarboxylation of its acidic precursor (CBGA) by exposure to heat or light. CBGA is also a precursor to the formation of CBCA, CBDA, and THCA. ${ }^{10}$ CBGA is an intermediate compound, immediately converted to either THCA or CBDA during the growth cycle and limiting the amount of CBGA and CBG found in mature Cannabis plants. Participants in this study were asked to use in-house analytical methods to determine the mass fraction (\%) of CBG and CBGA in the three hemp oils. The preparation of these hemp oils included a decarboxylation step resulting in extremely low levels of CBGA and levels of CBG consistent with normal ranges in commercial products.

\section{$\underline{\text { Reporting Statistics }}$}

- The enrollment and reporting statistics for CBG and CBGA are described in the table below for each analyte. Reported values may include non-quantitative results (zero or below LOQ).

\begin{tabular}{cccccccc} 
& \multicolumn{2}{c}{ Hemp Oil 1 } & \multicolumn{2}{c}{ Hemp Oil 2 } & \multicolumn{2}{c}{ Hemp Oil 2a } \\
& $\underline{\text { Number of }}$ & $\underline{\underline{\text { Reporting }}}$ & $\underline{\text { Number of }}$ & $\underline{\underline{\text { Reporting }}}$ & $\underline{\underline{\text { Number of }}}$ & $\underline{\underline{\text { Reporting }}}$ \\
$\underline{\text { Analyte }}$ & $\underline{\text { Participants }}$ & $\underline{\text { Results }}$ & $\underline{\text { Participants }}$ & $\underline{\text { Results }}$ & $\underline{\text { Participants }}$ & $\underline{\text { Results }}$ \\
CBG & 65 & $83 \%$ & 71 & $83 \%$ & 19 & $63 \%$ \\
CBGA & 63 & $70 \%$ & 73 & $68 \%$ & 19 & $47 \%$
\end{tabular}

- Most laboratories reported using solvent extraction or sample dilution for determination of CBG and CBGA in the three hemp oil samples (see table below). Additional sample preparation details are summarized at the end of the report in Appendix I.

\begin{tabular}{|c|c|c|}
\hline Reported & \multicolumn{2}{|c|}{ Percent Reporting } \\
\hline Preparation Method & $\underline{\mathrm{CBG}}$ & $\underline{\mathrm{CBGA}}$ \\
\hline Solvent Extraction & 70.5 & 73.9 \\
\hline Dilution & 21.2 & 21.0 \\
\hline Other & 0.0 & 0.0 \\
\hline None & 2.3 & 1.7 \\
\hline No Response & 6.1 & 3.4 \\
\hline
\end{tabular}

${ }^{10}$ M Zagozen, A Cerenak, S Kreft. Acta Pharm. 71: 355-364 (2021) https://doi.org/10.2478/acph-2021-0021 
- Most laboratories reported using LC-PDA or LC-UV for the determination of CBG and CBGA in the three hemp oil samples (see table below). Additional method details are summarized at the end of the report in Appendix I.

\begin{tabular}{ccc}
$\begin{array}{c}\text { Reported } \\
\text { Analytical Method }\end{array}$ & $\underline{\text { CBG }}$ & $\underline{\text { CBGA }}$ \\
\cline { 2 - 2 } LC-PDA & 63.6 & 64.7 \\
LC-UV & 26.5 & 29.4 \\
LC-MS & 2.3 & 3.4 \\
LC-MS/MS & 3.8 & 2.5 \\
GC-FID & 1.5 & 0.0 \\
GC-MS & 2.3 & 0.0 \\
Other & 0.0 & 0.0
\end{tabular}

$\underline{\text { Study Results }}$

$C B G$

- The mass fractions (\%) for CBG in the hemp oil samples were determined by NIST using LCPDA as described in Section 1 and are summarized in Table 6-1. These NIST values are used as the target means and ranges summarized in Table 6-2 for comparison to the participant results.

- The target and consensus means and ranges are summarized for CBG via different analytical methods in Figure 6-1, Figure 6-2, and Figure 6-3, which include data from laboratories submitting two or three results for CBG. Data from participants submitting only one measurement were included in Table 6-2 but were not included in the calculation of consensus statistics. ${ }^{2}$

- For CBG in Hemp Oil 1, the consensus range was based on quantitative results from 51 laboratories and completely overlaps within the target range (Figure 6-1).

- The individual laboratory means or thresholds from 25 laboratories (49\% of those reporting results) were outside the NIST range of tolerance for CBG in Hemp Oil 1.

- The individual laboratory means from 7 laboratories (14\% of those reporting results) were outside the acceptable $Z_{\text {comm }}^{\prime}$ score for CBG in Hemp Oil 1.

- Of the 2 laboratories reporting qualitative results, neither of the thresholds or LOQs were below the target mean for CBG in Hemp Oil 1.

- For CBG in Hemp Oil 2, the consensus range was based on quantitative results from 56 laboratories and completely overlaps within the target range (Figure 6-2).

- The individual laboratory means or thresholds from 23 laboratories $(41 \%$ of those reporting results) were outside the NIST range of tolerance for CBG in Hemp Oil 2.

- The individual laboratory means from 11 laboratories (20\% of those reporting results) were outside the acceptable $Z_{\text {comm }}^{\prime}$ score for CBG in Hemp Oil 2.

- The thresholds or LOQs for 1 of 2 laboratories reporting qualitative results were below the target mean for CBG in Hemp Oil 2.

- For CBG in Hemp Oil 2a, the consensus range was based on quantitative results from 10 laboratories and overlaps $50 \%$ of the target range (Figure 6-3). 
- The individual laboratory means or thresholds from 8 laboratories $(80 \%$ of those reporting results) were outside the NIST range of tolerance for CBG in Hemp Oil 2a.

- The individual laboratory mean from 2 laboratories (20\% of those reporting results) was outside the acceptable $Z_{\text {comm }}^{\prime}$ score for CBG in Hemp Oil 2a.

- The threshold or LOQ for 1 of 1 laboratory reporting qualitative results were below the target mean for CBG in Hemp Oil 2a.

- A comparison of individual laboratory means for CBG in Hemp Oil 1 and Hemp Oil 2 is summarized in Figure 6-4 for laboratories who reported results for both samples.

$C B G A$

- No target means or ranges were provided in Table 6-1 for CBGA in the three hemp oils.

- The consensus means and ranges for CBGA are based on quantitative data from 21 laboratories (Figure 6-5), 24 laboratories (Figure 6-6), and 3 laboratories (Figure 6-7) for Hemp Oil 1, Hemp Oil 2, and Hemp Oil 2a, respectively. Data from participants submitting only one measurement were included in Table 6-3 but were not included in the calculation of consensus statistics. $^{2}$

- A comparison of individual laboratory means for CBGA in Hemp Oil 1 and Hemp Oil 2 is summarized in Figure 6-8 for laboratories who reported results for both samples.

Overall

- The between-laboratory variabilities for determination of CBG and CBGA in the hemp oil samples are shown in the table below.

Between-Laboratory Variability (\% RSD)

\begin{tabular}{|c|c|c|c|}
\hline Analyte & Hemp Oil 1 & Hemp Oil 2 & Hemp Oil 2a \\
\hline $\mathrm{CBG}$ & 4.1 & 3.2 & 13.2 \\
\hline CBGA & 67.6 & 66.8 & 137.9 \\
\hline
\end{tabular}

Study Discussion and Technical Recommendations

The following recommendations are based on results obtained from the participants in this study.

$C B G$

- Approximately $27 \%$ of the laboratories reporting results for CBG provided values outside the consensus ranges for both Hemp Oil 1 and Hemp Oil 2 (Figure 6-4).

- Laboratories reporting results below the consensus mean in Hemp Oil 1 also reported results below the consensus mean for Hemp Oil 2. The reverse is also observed for laboratories reporting values above the consensus mean in both samples. Trends of this type often indicate a calibration bias.

- The between-laboratory variability was higher for CBG in Hemp Oil 2a (13.2 \%) than Hemp Oil $1(4.1 \%)$ and Hemp Oil 2 (3.2\%). The variability between individual mean laboratories were higher for CBG in Hemp Oil 1 (6.2 \%) in comparison to Hemp Oil 2 (4.8\%) and Hemp Oil 2a (4.0\%). 
- Hemp Oil 2a was prepared through a methanol/ethanol extraction of Hemp Oil 2 at NIST. The additional processing of Hemp Oil $2 \mathrm{a}$ was expected to result in minimal variability both within and among participating laboratories.

- The elevated level of variability may be an artifact of significantly fewer laboratories reporting results for Hemp Oil 2a (11) compared to Hemp Oil 1 (65) and Hemp Oil 2 (72).

- No additional trends were observed for the sample preparation, analytical methods, and/or analytical parameters reported for CBG in the three hemp oil samples.

$C B G A$

- Approximately $14 \%$ of the laboratories reporting results for CBGA provided values outside the consensus ranges for both Hemp Oil 1 and Hemp Oil 2 (Figure 6-8).

- Laboratories reporting results above the consensus mean in Hemp Oil 1 also reported results above the consensus mean for Hemp Oil 2. Trends of this type often indicate a calibration bias.

- No laboratories reported results below the consensus mean in Hemp Oil 1 or Hemp Oil 2 for CBGA.

- Most laboratories reported that CBGA was present in the samples at or below their LOQ (nonzero values). The low levels resulted in large consensus ranges and between-laboratory variabilities (67\% to $138 \%)$.

- Approximately $6 \%$ of the laboratories reporting results used LC-MS or LC-MS/MS methods did not have low enough LOQs to determine CBGA at the consensus level in Hemp Oil 1 and Hemp Oil 2; however, one laboratory had a low enough LOQ to determine CBGA at the consensus level in Hemp Oil 2a.

- Approximately $94 \%$ of the laboratories reporting results used LC-UV or LC-PDA methods with only $11 \%, 15 \%$, and $22 \%$ of these laboratories with low enough LOQs to determine CBGA at the consensus level in Hemp Oil 1, Hemp Oil 2, and Hemp Oil 2a, respectively.

- No additional trends were observed for the sample preparation, analytical methods, and/or analytical parameters reported for CBGA in the three hemp oil samples.

Overall

- Proper storage conditions are important for hemp oils because CBGA can readily convert to CBG when stored at elevated or room temperatures.

- Participants were asked to store the samples under controlled refrigeration $\left(\approx 4{ }^{\circ} \mathrm{C}\right)$.

- Laboratories should perform in-house evaluations of their storage conditions through stability test of their hemp oils at various environmental factors such as temperature, humidity, and light exposure..$^{5}$

- Over 100 cannabinoids have been identified in Cannabis plant samples with similarities in structure and molecular mass. As a result, chromatographic peak identity should always be confirmed using appropriate reference spectra of pure standards for cannabinoids and is particularly important for cannabinoids that are present at levels close to the method LOQ. Analytical methods should be able to clearly distinguish between cannabinoids.

- The use of appropriate calibration materials and quality assurance samples to establish that a method is in control and being performed correctly may reduce the likelihood of outlying data. Quality assurance samples can be commercially available reference materials (CRMs, SRMs, or RMs) or materials prepared in-house. 
- Measurement results should be reported accurately.

- Reported values should be the mass fraction (\%) of the CBG and CBGA in the three hemp oil samples. Participants who reported values based on a volume fraction (\%) for Hemp Oil 2 or Hemp Oil 2a should use the density of the sample for the conversion to mass fraction. Hemp Oil 1 was too viscous to prepare dilutions by volume.

- Zero is not a quantity that can be measured. If values are below LOQ, results should be reported as such. A more appropriate result would be to report that a value is below the LOQ (e.g., "< $<0.02 ")$.

- Laboratories reporting results based on a certain threshold should enter the numerical threshold (e.g., " $<1$ ").

- Laboratories reporting results flagged as outliers should check for calculation errors when preliminary data tables are sent for inspection. One example is to confirm that factors for all dilutions have been properly tabulated or that results are reported in the requested units. 
Table 6-1. Individualized data summary table (NIST) for CBG and CBGA in hemp oils.

\section{National Institute of Standards and Technology}

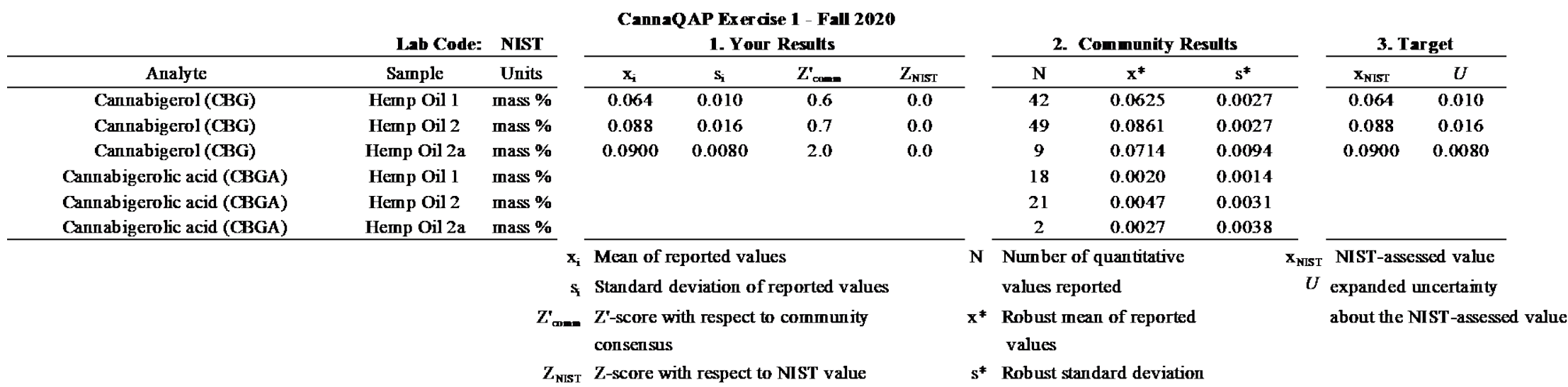


Table 6-2. Data summary table for CBG in hemp oils. Data highlighted in red have been flagged as a data entry of zero or results that include text (e.g., "<LOQ" or "present"). Data highlighted in blue have been identified as outside the consensus tolerance limits and would be estimated to result in an unacceptable $Z_{\text {comm }}^{\prime}$ score, $\left|Z_{\text {comm }}^{\prime}\right| \geq 2$. Note: This table spans two pages; the NIST values and consensus values are included on both pages for convenience.

\begin{tabular}{|c|c|c|c|c|c|c|c|c|c|c|c|c|c|c|c|c|}
\hline & \multirow[b]{3}{*}{ Lab } & \multicolumn{15}{|c|}{ Cannabigerol(CBG) } \\
\hline & & \multicolumn{5}{|c|}{ Heap Oil 1 (mass \%) } & \multicolumn{5}{|c|}{ He=p OAl 2 (mass \%) } & \multicolumn{5}{|c|}{ Help or 2 (mass \%) } \\
\hline & & $\mathbf{A}$ & $\mathbf{B}$ & $\mathbf{C}$ & Avg & SD & $\mathbf{A}$ & B & $\mathbf{C}$ & Avg & SD & $\mathbf{A}$ & $\mathbf{B}$ & $\mathbf{C}$ & Avg & SD \\
\hline \multirow{43}{*}{ 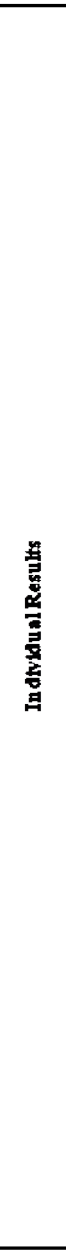 } & NIST & & & & 0.064 & 0.010 & & & & 0.088 & 0.016 & & & & 0.090 & 0.008 \\
\hline & $\mathrm{A} 002$ & 0.06885 & 0.06925 & 0.04051 & 0.0595 & 0.0165 & 0.081143 & 0.077166 & 0.077195 & 0.0785 & 0.0023 & & & & & \\
\hline & A003 & & & & & & 0.107 & 0.106 & 0.083 & 0.0987 & 0.0136 & & & & & \\
\hline & A 005 & 0.0761 & 0.072 & 0.0783 & 0.0755 & 0.0032 & 0.119 & 0.0997 & 0.11 & 0.1096 & 0.0097 & & & & & \\
\hline & A006 & 0.08 & & & 0.0800 & & 0.11 & & & 0.1100 & & & & & & \\
\hline & A 007 & & & & & & & & & & & & & & & \\
\hline & A 008 & 0.041 & & & 0.0410 & & & & & & & & & & & \\
\hline & A 009 & & & & & & & & & & & $<0.00219$ & $<0.00219$ & $<0.00219$ & $<0.00219$ & \\
\hline & A010 & & & & & & & & & & & present & present & present & & \\
\hline & $\mathrm{A} 012$ & & & & & & 0.08452 & 0.08509 & 0.08495 & 0.0849 & 0.0003 & & & & & \\
\hline & A013 & 0.063 & 0.061 & 0.067 & 0.0637 & 0.0031 & 0.111 & 0.121 & 0.12 & 0.1173 & 0.0055 & & & & & \\
\hline & A 014 & $<0.09$ & & & $<0.09$ & & 0.15 & & & 0.1500 & & & & & & \\
\hline & A015 & & & & & & & & & & & 01 & 0.14 & 0.15 & 0.1300 & 0.0265 \\
\hline & A 016 & & & & & & & & & & & & & & & \\
\hline & A017 & 0.07 & & & 0.0700 & & 0.09 & & & 0.0900 & & & & & & \\
\hline & A 018 & & & & & & & & & & & & & & & \\
\hline & A019 & $<0.09$ & & & $<0.09$ & & $<0.09$ & & & $<0.09$ & & & & & & \\
\hline & $\mathrm{A} 020$ & 0.054465 & 0.055098 & 0.054253 & 0.0546 & 0.0004 & 0.075415 & 0.072179 & 0.076533 & 0.0747 & 0.0023 & & & & & \\
\hline & A021 & & & & & & & & & & & 0.06 & 0.07 & 0.07 & 0.0667 & 0.0058 \\
\hline & $\mathrm{A} 022$ & & & & & & & & & & & 0.0556 & 0.0561 & 0.0557 & 0.0558 & 0.0003 \\
\hline & A023 & & & & & & 0.0866 & 0.0874 & 0.0885 & 0.0875 & 0.0010 & & & & & \\
\hline & A 024 & & & & & & & & & & & 0.092 & 0.091 & 0.092 & 0.0917 & 0.0006 \\
\hline & A025 & & & & & & 0.07 & 0.06 & 0.06 & 0.0633 & 0.0058 & & & & & \\
\hline & $\mathrm{A} 027$ & & & & & & & & & & & 0.083 & 0.084 & 0.0834 & 0.0835 & 0.0005 \\
\hline & A030 & 0.116 & & & 0.1160 & & 0.11 & & & 0.1100 & & & & & & \\
\hline & A 031 & & & & & & & & & & & & & & & \\
\hline & A033 & 0.06 & 0.07 & 0.07 & 0.0667 & 0.0058 & 0.07 & 0.08 & 0.08 & 0.0767 & 0.0058 & & & & & \\
\hline & A 035 & 0.0666 & & & 0.0666 & & 0.0828 & 0.0866 & 0.0832 & 0.0842 & 0.0021 & & & & & \\
\hline & A036 & 0.061 & 0.06 & 0.062 & 0.0610 & 0.0010 & 0.08 & 0.08 & 0.084 & 0.0813 & 0.0023 & & & & & \\
\hline & A 037 & 0.063 & 0.063 & 0.061 & 0.0623 & 0.0012 & 0.0881 & 0.0911 & 0.0873 & 0.0888 & 0.0020 & & & & & \\
\hline & A038 & 00668 & 0.069 & 0.0693 & 0.0684 & 0.0014 & 0.0935 & 0.0878 & 0.0885 & 0.0899 & 0.0031 & & & & & \\
\hline & A 039 & 0.06 & 0.05 & 0.08 & 0.0633 & 0.0153 & 0.33 & 0.34 & 0.35 & 0.3400 & 0.0100 & & & & & \\
\hline & A040 & & & & & & & & & & & 0.05905 & 0.05952 & 0.05543 & 0.0580 & 0.0022 \\
\hline & A 041 & 0.1 & 0.1 & 0.1 & 0.1000 & 0.0000 & 0.1 & 0.1 & 0.1 & 0.1000 & 0.0000 & & & & & \\
\hline & A 043 & 0.095 & 0.097 & 0.097 & 0.0963 & 0.0012 & 0.189 & 0.188 & 0.187 & 0.1880 & 0.0010 & & & & & \\
\hline & A 044 & & & & & & & & & & & & & & & \\
\hline & A045 & & & & & & & & & & & & & & & \\
\hline & A 046 & 0 & 0 & 0 & 0.0000 & 0.0000 & 0 & 0 & 0 & 0.0000 & 0.0000 & & & & & \\
\hline & A050 & 0.0625 & 0.064 & 0.0628 & 0.0631 & 0.0008 & 0.0849 & 0.0846 & 0.0848 & 0.0848 & 0.0002 & & & & & \\
\hline & A 052 & & & & & & & & & & & & & & & \\
\hline & A 053 & & & & & & & & & & & & & & & \\
\hline & A 054 & $<0.02$ & $<0.02$ & 0.03 & 0.0300 & & $<0.02$ & 0.04 & $<0.02$ & 0.0400 & & & & & & \\
\hline & A055 & 0.0546 & 0.0602 & 0.0545 & 0.0564 & 0.0033 & 0.073 & 0.0803 & 0.0728 & 0.0754 & 0.0043 & & & & & \\
\hline 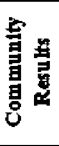 & & $\begin{array}{l}\text { Consensu } \\
\text { Consensu } \\
\text { Maximum } \\
\text { Minimum } \\
\mathrm{N}\end{array}$ & $\begin{array}{l}\text { Is Mean } \\
\text { Is Standard I } \\
\text { n }\end{array}$ & Deviation & $\begin{array}{c}0.0629 \\
0.0026 \\
0.1263 \\
0.0000 \\
42 \\
\end{array}$ & & $\begin{array}{l}\text { Consensus } \\
\text { Consensus } \\
\text { Maximum } \\
\text { Minimum } \\
\mathrm{N}\end{array}$ & $\begin{array}{l}\text { s Mean } \\
\text { s Standard }\end{array}$ & Deviation & $\begin{array}{c}0.0861 \\
0.0027 \\
0.3400 \\
0.0000 \\
49 \\
\end{array}$ & & $\begin{array}{l}\text { Consensu } \\
\text { Consensu: } \\
\text { Maximum } \\
\text { Minimum } \\
\mathrm{N}\end{array}$ & $\begin{array}{l}\text { s Mean } \\
\text { s Stmandand I }\end{array}$ & Deviation & $\begin{array}{c}0.0714 \\
0.0094 \\
0.1300 \\
0.0000 \\
9\end{array}$ & \\
\hline
\end{tabular}




\begin{tabular}{|c|c|c|c|c|c|c|c|c|c|c|c|c|c|c|c|c|c|}
\hline & & & & & & & & & Cann & nabigerol(c) & CBG & & & & & & \\
\hline & & & & Heap & Oil 1 (n) & ss) & & & He=p & p ON2(na & ss \%) & & & He= & Dil 2a (n & ss \%) & \\
\hline & & Lab & $\mathbf{A}$ & B & $\mathbf{C}$ & Avg & SD & $\boldsymbol{A}$ & B & $\mathbf{C}$ & Avg & SD & $\boldsymbol{A}$ & B & $\mathbf{C}$ & Avg & SD \\
\hline & & NIST & & & & 0.064 & 0.010 & & & & 0.088 & 0.016 & & & & 0.090 & 0.008 \\
\hline & & A 056 & & & & & & & & & & & & & & & \\
\hline & & A057 & & & & & & & & & & & & & & & \\
\hline & & A 058 & 0.133 & 0.123 & 0.123 & 0.1263 & 0.0058 & 0.125 & 0.127 & 0.139 & 0.1303 & 0.0076 & & & & & \\
\hline$\rightarrow$ & & A059 & 0.082 & 0.081 & 0.083 & 0.0820 & 0.0010 & 0.112 & 0.108 & 0.11 & 0.1100 & 0.0020 & & & & & \\
\hline 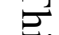 & & $\mathrm{A} 060$ & 0.06 & 0.06 & 0.06 & 0.0600 & 0.0000 & 0.08 & 0.08 & 0.09 & 0.0833 & 0.0058 & & & & & \\
\hline$\vec{\sim}$ & & A 061 & 0.098 & 0.098 & 0.097 & 0.0977 & 0.0006 & 0.141 & 0.119 & 0.139 & 0.1330 & 0.0122 & & & & & \\
\hline$\nabla$ & & A 062 & & & & & & & & & & & 0.069 & 0.07 & 0.07 & 0.0697 & 0.0006 \\
\hline 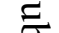 & & A063 & & & & & & 0.08707 & 0.09317 & 0.09053 & 0.0903 & 0.0031 & & & & & \\
\hline$\Xi$ & & A 064 & & & & & & & & & & & & & & & \\
\hline 8 & & A 066 & yes & yes & yes & & & & & & & & & & & & \\
\hline$\stackrel{0}{=}$ & & A 068 & & & & & & & & & & & & & & & \\
\hline 0 & & $A 071$ & 0 & & & 0.0000 & & 0 & & & 00000 & & & & & & \\
\hline$\Xi$ & & A 072 & & & & & & & & & & & & & & & \\
\hline$\vec{\sigma}$. & & A073 & 0.071 & 0.076 & 0.068 & 0.0717 & 0.0040 & 0.103 & 0.103 & 0.103 & 0.1030 & 0.0000 & & & & & \\
\hline ט & & A 074 & 0.061 & 0.058 & 0.06 & 0.0597 & 0.0015 & 0.077 & 0.079 & 0.077 & 0.0777 & 0.0012 & & & & & \\
\hline$\overline{1}$ & & A 075 & & & & & & & & & & & & & & & \\
\hline き. & & A 076 & 0.001763 & 0.001818 & 0.001779 & 0.0018 & 0.0000 & 0.002148 & 0.002114 & 0.002057 & 0.0021 & 0.0000 & & & & & \\
\hline D & & A.077 & & & & & & $<\mathrm{IOQ}$ & $<\mathrm{IOQ}$ & $\varangle \mathrm{LOQ}$ & & & & & & & \\
\hline$\tilde{\sigma}$ & & A 081 & 0 & 0 & 0 & 0.0000 & 0.0000 & 0 & 0 & 0 & 0.0000 & 0.0000 & & & & & \\
\hline$\vec{D}$ & & A 082 & & & & & & & & & & & 0 & & & 0.0000 & \\
\hline E & & A 083 & 0.06 & & & 0.0600 & & 0.08 & & & 0.0800 & & & & & & \\
\hline 8 & & A084 & 0.057 & 0.064 & 0.059 & 0.0600 & 0.0036 & 0.086 & 0.079 & 0.08 & 0.0817 & 0.0038 & & & & & \\
\hline 0 & 寻 & A 085 & 0.07 & 0.07 & 0.07 & 0.0700 & 0.0000 & 0.08 & 0.08 & 0.08 & 0.0800 & 0.0000 & & & & & \\
\hline$\stackrel{0}{\rightarrow}$ & $\stackrel{g}{g}$ & A087 & & & & & & & & & & & & & & & \\
\hline$\rho$ & $\vec{z}$ & A 088 & & & & & & 0.08 & 0.08 & 0.09 & 0.0833 & 0.0058 & & & & & \\
\hline $\overrightarrow{0}$ & 3 & A089 & 0.06 & 0.06 & 0.08 & 0.0667 & 0.0115 & 0.08 & 0.08 & 0.08 & 0.0800 & 0.0000 & & & & & \\
\hline$\underset{0}{\mathscr{0}}$ & 步 & A 090 & & & & & & & & & & & 0.0555 & 0.056 & 0.056 & 0.0558 & 0.0003 \\
\hline D & & A091 & & & & & & & & & & & & & & & \\
\hline$\Rightarrow$ & & A 092 & 0.0569 & 0.0566 & 0.0564 & 0.0566 & 0.0003 & 0.0724 & 0.0724 & 0.0725 & 0.0724 & 0.0001 & & & & & \\
\hline $0^{\circ}$ & & $\mathbf{A} 093$ & 0.07 & 0.07 & 0.07 & 0.0700 & 0.0000 & 0.09 & 0.09 & $0 \_07$ & 0.0833 & 0.0115 & & & & & \\
\hline 当 & & A 095 & 0.0936 & 0.0644 & 0.0971 & 0.0850 & 0.0180 & 0.0903 & 0.0933 & 0.1249 & 0.1028 & 0.0192 & & & & & \\
\hline 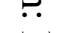 & & A 096 & & & & & & & & & & & & & & & \\
\hline$\Xi$ & & A 098 & 0.074 & & & 0.0740 & & $<0.0025$ & & & $<0.0025$ & & & & & & \\
\hline & & A099 & 0.063 & 0.062 & 0.063 & 0.0627 & 0.0006 & 0.088 & 0.086 & 0.082 & 0.0853 & 0.0031 & & & & & \\
\hline 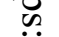 & & A 100 & 0.04314 & 0.04471 & 0.04673 & 0.0449 & 0.0018 & 0.0707 & 0.06797 & 0.06874 & 0.0691 & 0.0014 & & & & & \\
\hline 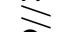 & & A 101 & & & & & & & & & & & & & & & \\
\hline ? & & A 102 & 0.06 & 0.05 & 0.05 & 0.0533 & 0.0058 & 0.08 & 0.08 & 0.08 & 0.0800 & 0.0000 & & & & & \\
\hline$\stackrel{0}{0}$ & & A.103 & 0 & 0 & 0 & 0.0000 & 0.0000 & 0.068 & 0.083 & 0.076 & 0.0757 & 0.0075 & & & & & \\
\hline$\dot{0}$ & & A 104 & 0.051 & 0.048 & 0.052 & 0.0503 & 0.0021 & 0.053 & 0.053 & 0.06 & 0.0553 & 0.0040 & & & & & \\
\hline बें & & A 105 & 0.12 & 0.09 & 0.09 & 0.1000 & 0.0173 & 0.11 & 0.12 & 0.12 & 0.1167 & 0.0058 & & & & & \\
\hline 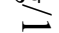 & & A 106 & 0.064 & 0.063 & 0.062 & 0.0630 & 0.0010 & 0.089 & 0.087 & 0.086 & 0.0873 & 0.0015 & & & & & \\
\hline$\overline{0}$ & & A 107 & 0.0564 & 0.0527 & 0.0546 & 0.0546 & 0.0019 & 0.0691 & 0.0667 & 0.0654 & 0.0671 & 0.0019 & & & & & \\
\hline o & & A 108 & & & & & & & & & & & & & & & \\
\hline 0 & & A 109 & 0.07 & 0.06 & & 0.0650 & 0.0071 & 0.1 & 0.08 & & 00900 & 0.0141 & & & & & \\
\hline$\infty$ & & A 110 & 0.06 & 0.06 & 0.06 & 0.0600 & 0.0000 & 0.07 & 0.07 & 0.07 & 0.0700 & 0.0000 & & & & & \\
\hline 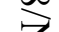 & & A112 & 0.0402 & 0.0423 & 0.0413 & 0.0413 & 0.0011 & 0.0486 & 0.0447 & 0.0467 & 0.0467 & 0.0020 & & & & & \\
\hline$\angle$ & & A 113 & 0.048092 & 0.047682 & 0.048611 & 0.0481 & 0.0005 & 0 & 0 & 0 & 0.0000 & 0.0000 & & & & & \\
\hline$\pi$ & & A114 & 0.0679 & 0.0674 & 0.0644 & 0.0666 & 0.0019 & 0.086 & 0.0888 & 0.0899 & 00882 & 0.0020 & & & & & \\
\hline$\Rightarrow$ & & A 115 & & & & & & & & & & & 0.09 & 0.09 & 0.09 & 0.0900 & 0.0000 \\
\hline 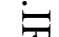 & & A116 & 0.0276 & 0.0599 & 0.0584 & 0.0486 & 0.0182 & 0.0598 & 0.0865 & 0.0831 & 0.0765 & 0.0145 & & & & & \\
\hline$\infty_{\infty}^{\infty}$ & 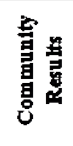 & & \begin{tabular}{|l} 
Consensu \\
Consensu \\
Maximum \\
Minimum \\
$\mathrm{N}$
\end{tabular} & $\begin{array}{l}\text { is Mean } \\
\text { s Standard } \\
\text { n }\end{array}$ & Deviation & $\begin{array}{c}0.0629 \\
0.0026 \\
0.1263 \\
0.0000 \\
42\end{array}$ & & \begin{tabular}{|l} 
Consensu: \\
Consensus \\
Maximum \\
Minimum \\
$\mathrm{N}$
\end{tabular} & $\begin{array}{l}\text { s Mean } \\
\text { s Standard }\end{array}$ & Deviation & $\begin{array}{c}0.0861 \\
0.0027 \\
0.3400 \\
0.0000 \\
49\end{array}$ & & \begin{tabular}{|l} 
Consensu \\
Consensu \\
Maximum \\
Minimum \\
$\mathrm{N}$
\end{tabular} & $\begin{array}{l}\text { Mean } \\
\text { Standand }\end{array}$ & eviation & $\begin{array}{c}0.0714 \\
0.0094 \\
0.1300 \\
0.0000 \\
9\end{array}$ & \\
\hline
\end{tabular}




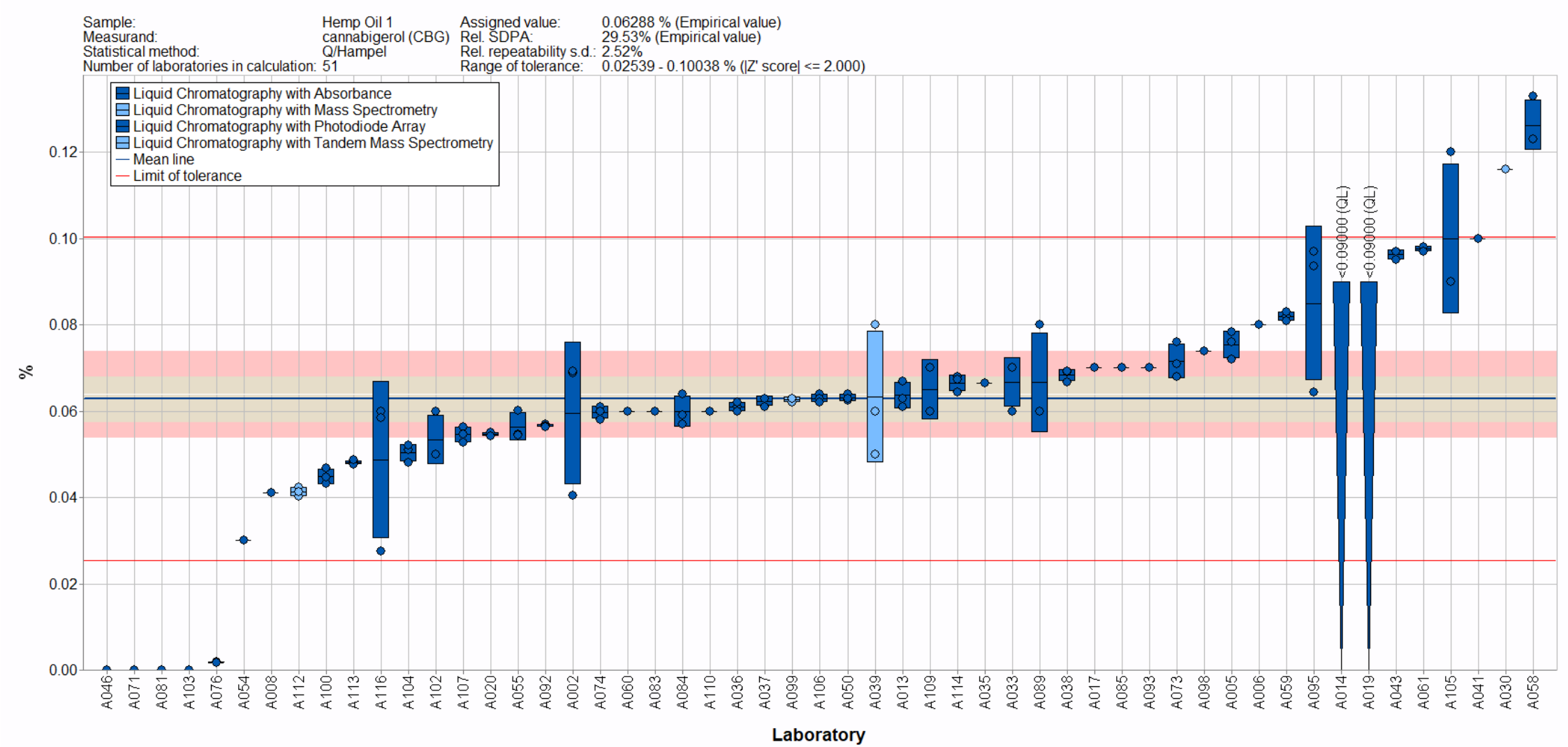

Figure 6-1. CBG in Hemp Oil 1 (data summary view - analytical method). In this view, individual laboratory data are plotted (circles) with the individual laboratory standard deviation (rectangle). The color of the data point represents the analytical method employed. The solid blue line represents the consensus mean, and the green shaded region represents the $95 \%$ confidence interval for the consensus mean. The solid red lines represent the consensus range of tolerance, calculated as the values above and below the consensus mean that result in an acceptable $Z_{\text {comm }}^{\prime}$ score, $\left|Z_{\text {comm }}^{\prime}\right| \leq 2$. The red shaded region represents the NIST range of tolerance, which encompasses the target value bounded by twice its uncertainty ( $U_{\text {NIST }}$ ) and represents the range that results in an acceptable $Z_{\text {NIST }}$ score, $\left|Z_{\text {NIST }}\right| \leq 2$. The shaded beige region represents the overlapping of the $95 \%$ confidence interval for the consensus mean (green region) and the NIST range of tolerance (red region). The downward triangle represents data reported as a threshold or LOQ value. 


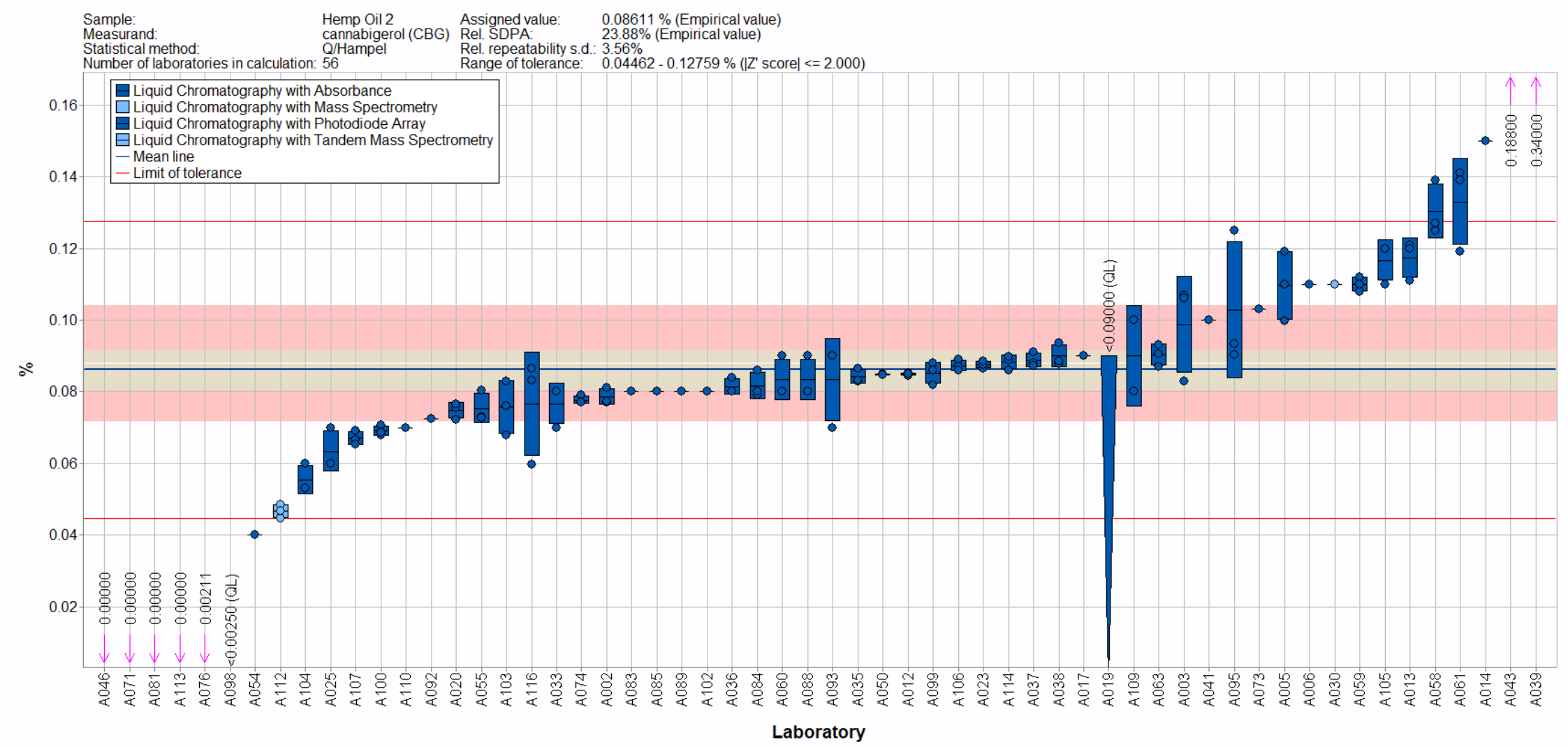

Figure 6-2. CBG in Hemp Oil 2 (data summary view - analytical method). In this view, individual laboratory data are plotted (circles) with the individual laboratory standard deviation (rectangle). The color of the data point represents the analytical method employed. The solid blue line represents the consensus mean, and the green shaded region represents the $95 \%$ confidence interval for the consensus mean. The solid red lines represent the consensus range of tolerance, calculated as the values above and below the consensus mean that result in an acceptable $Z_{\text {comm }}^{\prime}$ score, $\left|Z_{\text {comm }}^{\prime}\right| \leq 2$. The red shaded region represents the NIST range of tolerance, which encompasses the target value bounded by twice its uncertainty ( $U_{\text {NIST }}$ ) and represents the range that results in an acceptable $Z_{\text {NIST }}$ score, $\left|Z_{\text {NIST }}\right| \leq 2$. The shaded beige region represents the overlapping of the $95 \%$ confidence interval for the consensus mean (green region) and the NIST range of tolerance (red region). The downward triangle represents data reported as a threshold or LOQ value. 


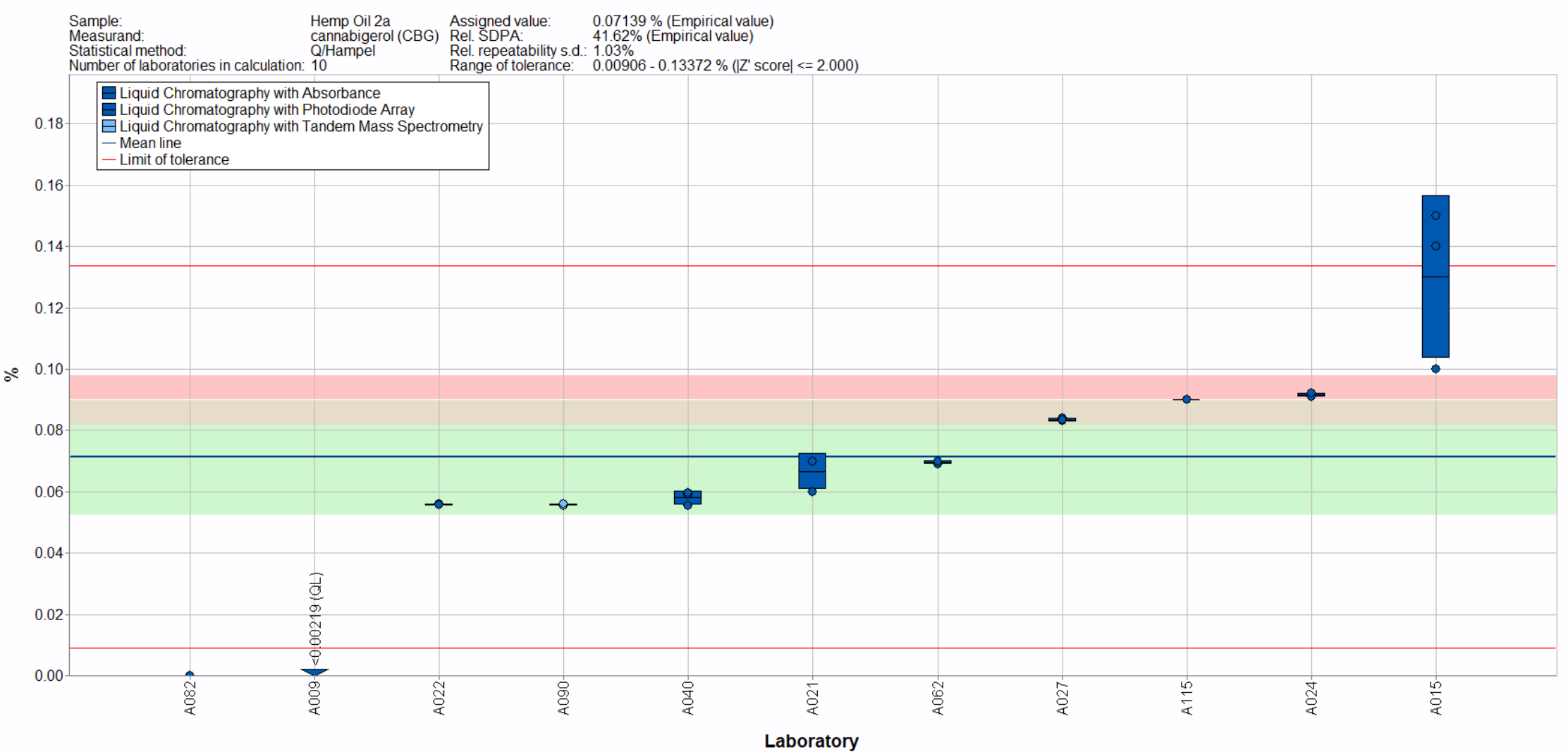

Figure 6-3. CBG in Hemp Oil 2a (data summary view - analytical method). In this view, individual laboratory data are plotted (circles) with the individual laboratory standard deviation (rectangle). The color of the data point represents the analytical method employed. The solid blue line represents the consensus mean, and the green shaded region represents the $95 \%$ confidence interval for the consensus mean. The solid red lines represent the consensus range of tolerance, calculated as the values above and below the consensus mean that result in an acceptable $Z_{\text {comm }}^{\prime}$ score, $\left|Z_{\text {comm }}^{\prime}\right| \leq 2$. The red shaded region represents the NIST range of tolerance, which encompasses the target value bounded by twice its uncertainty ( $U_{\text {NIST }}$ ) and represents the range that results in an acceptable $Z_{\text {NIST }}$ score, $\left|Z_{\text {NIST }}\right| \leq 2$. The shaded beige region represents the overlapping of the $95 \%$ confidence interval for the consensus mean (green region) and the NIST range of tolerance (red region). The downward triangle represents data reported as a threshold or LOQ value. 


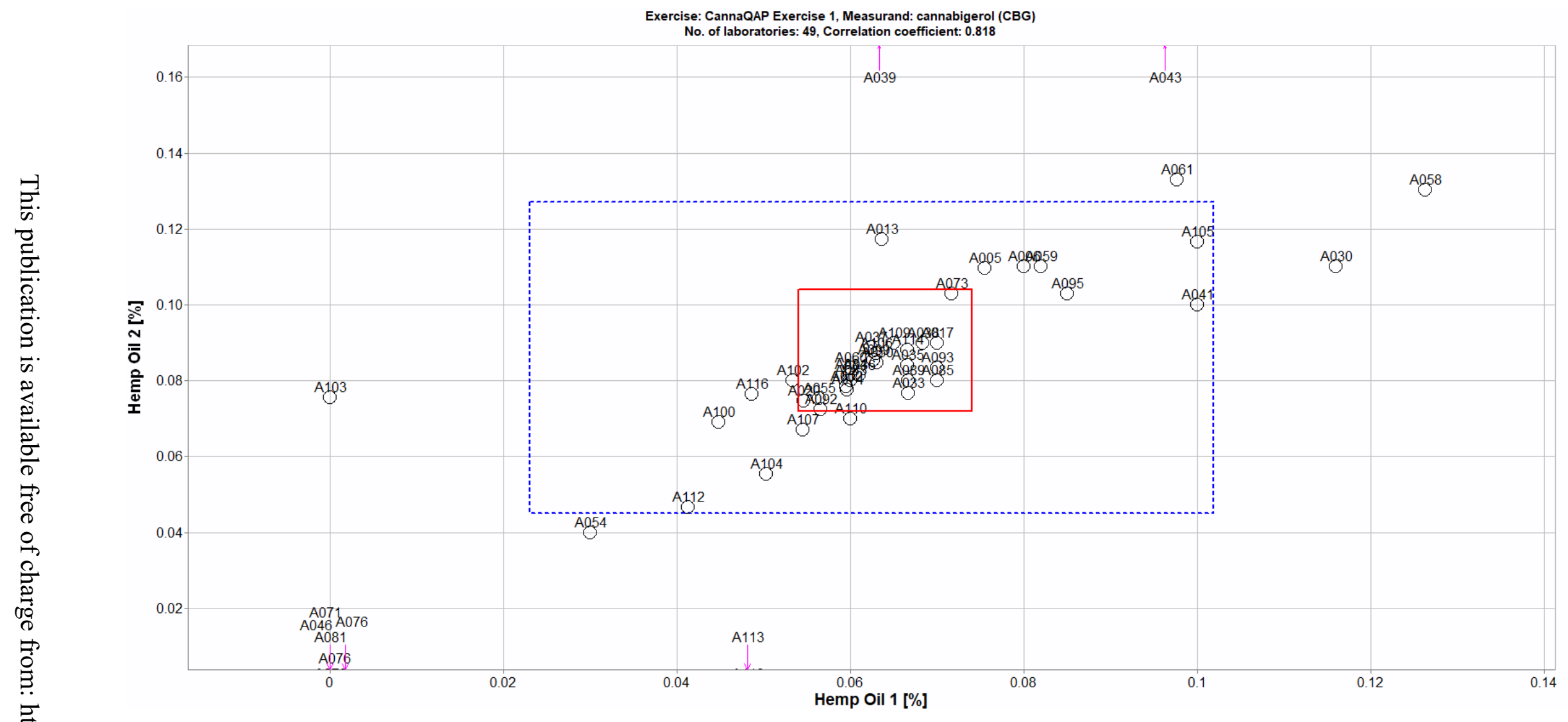

Figure 6-4. Laboratory means for CBG in Hemp Oil 1 and Hemp Oil 2 (sample/sample comparison view). In this view, the individual laboratory mean for one sample (Hemp Oil 1) is compared to the individual laboratory mean for a second sample (Hemp Oil 2). The solid red box represents the NIST range of tolerance for the two samples, Hemp Oil 1 (x-axis) and Hemp Oil 2 (y-axis), which encompasses the target values bounded by their uncertainties ( $\left.U_{\text {NIST }}\right)$ and represents the range that results in an acceptable $Z_{\text {NIST }}$ score, $\left|Z_{\text {NIST }}\right| \leq 2$. The dotted blue box represents the consensus range of tolerance for Hemp Oil 1 (x-axis) and Hemp Oil 2 (y-axis), calculated as the values above and below the consensus means that result in an acceptable $Z_{\text {comm }}^{\prime}$ score, $\left|Z_{\text {comm }}^{\prime}\right| \leq 2$. 
Table 6-3. Data summary table for CBA in hemp oils. Data highlighted in red have been flagged as a data entry of zero or results that include text (e.g., "< LOQ" or "present"). Data highlighted in blue have been identified as outside the consensus tolerance limits and would be estimated to result in an unacceptable $Z_{\text {comm }}^{\prime}$ score, $\left|Z_{\text {comm }}^{\prime}\right| \geq 2$. Note: This table spans two pages; the NIST values and consensus values are included on both pages for convenience.

\begin{tabular}{|c|c|c|c|c|c|c|c|c|c|c|c|c|c|c|c|c|}
\hline & \multirow[b]{3}{*}{ Lab } & \multicolumn{15}{|c|}{ Cannabigerolic acid (CBGA) } \\
\hline & & \multicolumn{10}{|c|}{ Heap 012 (mass \%) } & \multicolumn{5}{|c|}{ Henp 0123 (-nass \%) } \\
\hline & & $\mathbf{A}$ & $\mathbf{B}$ & $\mathbf{c}$ & Avg & SD & $\mathbf{A}$ & $\mathbf{B}$ & $\mathbf{C}$ & Avg & SD & $\mathbf{A}$ & $\mathbf{B}$ & $\mathbf{c}$ & Avg & SD \\
\hline \multirow{43}{*}{ 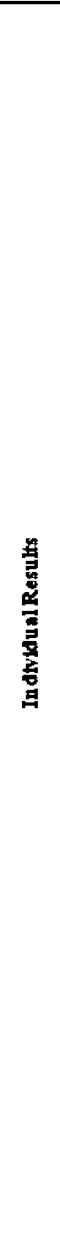 } & NIST & & & & & & & & & & & & & & & \\
\hline & $\mathrm{A} 002$ & $<0.0049$ & $<0.0049$ & $<0.0049$ & $<0.0049$ & & $<0.0049$ & $<0.0049$ & $<0.0049$ & $<0.0049$ & & & & & & \\
\hline & A003 & & & & & & $<0.01$ & $<0.01$ & $<0.01$ & $<0.01$ & & & & & & \\
\hline & $\mathrm{A} 005$ & 0 & 0 & 0 & 0.0000 & 0.0000 & 0 & 0 & 0 & 0.0000 & 0.0000 & & & & & \\
\hline & A006 & 0.02 & & & 0.0200 & & 0.07 & & & 0.0700 & & & & & & \\
\hline & A 007 & & & & & & & & & & & & & & & \\
\hline & A008 & & & & & & & & & & & & & & & \\
\hline & $\mathrm{A} 009$ & & & & & & & & & & & $<0.00192$ & $<0.00192$ & $<0.00192$ & $<0.00192$ & \\
\hline & A010 & & & & & & & & & & & & & & & \\
\hline & $\mathrm{A} 012$ & & & & & & 0 & 0 & 0 & 0.0000 & 0.0000 & & & & & \\
\hline & A013 & & & & & & & & & & & & & & & \\
\hline & A 014 & $<0.09$ & & & $<0.09$ & & $<0.09$ & & & $<0.09$ & & & & & & \\
\hline & A015 & & & & & & & & & & & & & & & \\
\hline & A 016 & & & & & & & & & & & & & & & \\
\hline & A017 & 0.01 & & & 0.0100 & & 0.03 & & & 0.0300 & & & & & & \\
\hline & A 018 & & & & & & & & & & & & & & & \\
\hline & A019 & $<0.09$ & & & $<009$ & & $<009$ & & & $<009$ & & & & & & \\
\hline & A 020 & & & & & & & & & & & & & & & \\
\hline & A021 & & & & & & & & & & & $<0.05$ & $<0.05$ & $<0.05$ & $<0.05$ & \\
\hline & $\mathrm{A} 022$ & & & & & & & & & & & & & & & \\
\hline & A023 & & & & & & 0 & 0 & 0 & 0.0000 & 0.0000 & & & & & \\
\hline & A 024 & & & & & & & & & & & $<0.010$ & $<0.010$ & $<0.010$ & $<0.010$ & \\
\hline & A025 & & & & & & & & & & & & & & & \\
\hline & $\mathrm{A} 027$ & & & & & & & & & & & $\mid<0.0057$ & $<0.0057$ & $<0.0057$ & $<0.0057$ & \\
\hline & A030 & $<0.01$ & & & $<0.01$ & & $<0.01$ & & & $<0.01$ & & & & & & \\
\hline & $\mathrm{A} 031$ & & & & & & & & & & & & & & & \\
\hline & A033 & 0 & 0 & 0 & 0.0000 & 0.0000 & 0 & 0 & 0 & 0.0000 & 0.0000 & & & & & \\
\hline & A 035 & $<0.00250$ & & & $<0.00250$ & & $<0.0025$ & $<0.0025$ & $<0.0025$ & $<0.0025$ & & & & & & \\
\hline & A036 & $<0206$ & $<0.206$ & $<0206$ & $<0206$ & & $<0247$ & $<0.247$ & $<0247$ & $<0247$ & & & & & & \\
\hline & A 037 & $<0.05$ & $<0.05$ & $<0.05$ & $<0.05$ & & $<0.05$ & $<0.05$ & $<0.05$ & $<0.05$ & & & & & & \\
\hline & A038 & $<0.025$ & $<0.025$ & $<0.025$ & $<0.025$ & & $<0.025$ & $<0.025$ & $<0.025$ & $<0.025$ & & & & & & \\
\hline & A 039 & 0 & 0 & 0 & 0.0000 & 0.0000 & 0 & 0 & 0 & 0.0000 & 0.0000 & & & & & \\
\hline & A040 & & & & & & & & & & & \begin{tabular}{|l|l}
0.00798 \\
\end{tabular} & 0.00845 & 0.00775 & 0.0081 & 0.0004 \\
\hline & A 041 & 0 & 0 & 0 & 0.0000 & 0.0000 & 0 & 0 & 0 & 0.0000 & 0.0000 & & & & & \\
\hline & A043 & 0.008 & 0.008 & 0.007 & 0.0077 & 0.0006 & 0.018 & 0.024 & 0018 & 0.0200 & 0.0035 & & & & & \\
\hline & A 044 & & & & & & & & & & & & & & & \\
\hline & A045 & & & & & & & & & & & & & & & \\
\hline & A 046 & 0 & 0 & 0 & 0.0000 & 0.0000 & 0 & 0 & 0 & 0.0000 & 0.0000 & & & & & \\
\hline & A050 & $<0.01$ & $<0.01$ & $<0.01$ & $<0.01$ & & $<0.01$ & $<0.01$ & $<0.01$ & $<0.01$ & & & & & & \\
\hline & $\mathrm{A} 052$ & & & & & & & & & & & & & & & \\
\hline & A053 & & & & & & & & & & & & & & & \\
\hline & A 054 & $<0.01$ & $<0.01$ & $<0.01$ & $<0.01$ & & $<0.01$ & $<0.01$ & $<0.01$ & $<0.01$ & & & & & & \\
\hline & A055 & 0 & 0 & 0 & 0.0000 & 0.0000 & 0 & 0 & 0 & 0.0000 & 0.0000 & & & & & \\
\hline 焉 & & \begin{tabular}{|l} 
Consens \\
Consens \\
Maximum \\
Minimum \\
N
\end{tabular} & $\begin{array}{l}\text { Mean } \\
\text { s Standard }\end{array}$ & Deviation & $\begin{array}{c}0.0020 \\
0.0014 \\
0.0367 \\
0.0000 \\
18\end{array}$ & & $\begin{array}{l}\text { Consensu } \\
\text { Consensu } \\
\text { Maximm } \\
\text { Minimum } \\
\text { N }\end{array}$ & $\begin{array}{l}\text { s Mean } \\
\text { s Standard }\end{array}$ & Deviation & $\begin{array}{c}0.0047 \\
0.0031 \\
0.1033 \\
0.0000 \\
21\end{array}$ & & \begin{tabular}{|l|} 
Consensu: \\
Consensu \\
Maximum \\
Minimmm \\
N
\end{tabular} & $\begin{array}{l}\text { Is Mean } \\
\text { s Standand }\end{array}$ & Deviation & $\begin{array}{c}0.0027 \\
0.0038 \\
0.0081 \\
0.0000 \\
2\end{array}$ & \\
\hline
\end{tabular}




\begin{tabular}{|c|c|c|c|c|c|c|}
\hline & 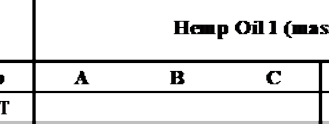 & 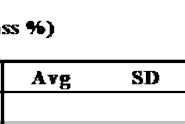 & 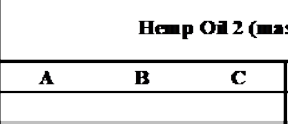 & 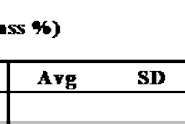 & 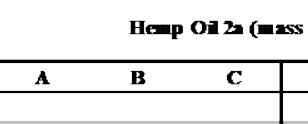 & 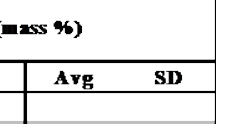 \\
\hline & simang & & aim a da & & & \\
\hline & & & 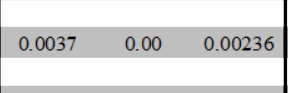 & tion a & & \\
\hline & & & & & & \\
\hline & 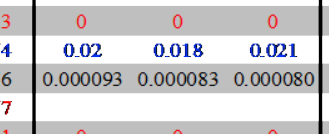 & taty ans & 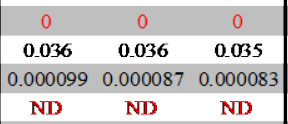 & 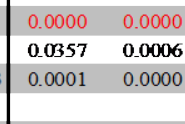 & & \\
\hline & - & sals & 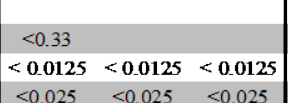 & Tals & & |axos \\
\hline & 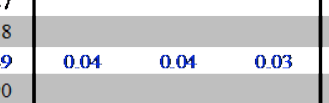 & Jang ans & 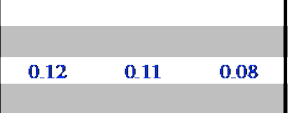 & oim $\cos x$ & tomat aeve & 1 \\
\hline & 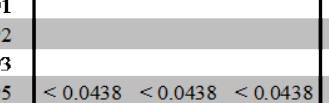 & 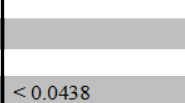 & 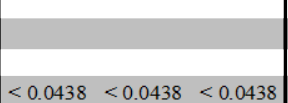 & Ioment & & 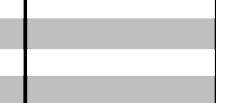 \\
\hline & 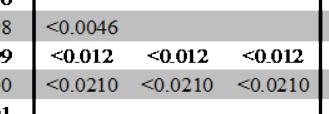 & 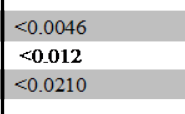 & 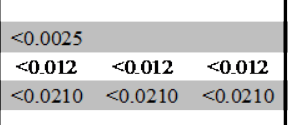 & sals & & 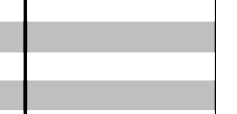 \\
\hline & 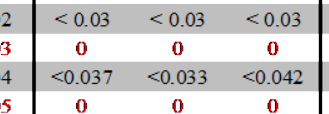 & 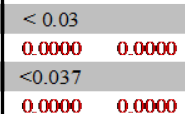 & 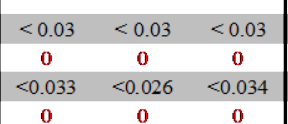 & $=$ & & - \\
\hline & 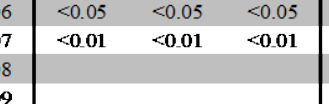 & som & 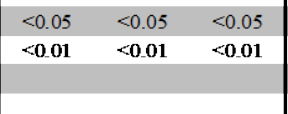 & sil & & \\
\hline & -2 & 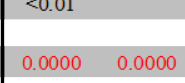 & 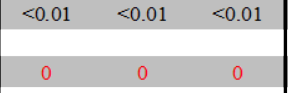 & 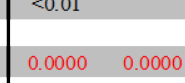 & & - \\
\hline & & & 20 & & $=$ & \\
\hline II & 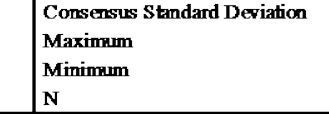 & 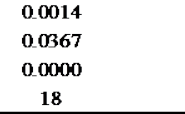 & $=$ & 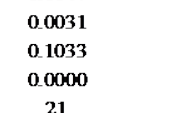 & $=$ & 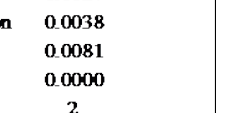 \\
\hline
\end{tabular}




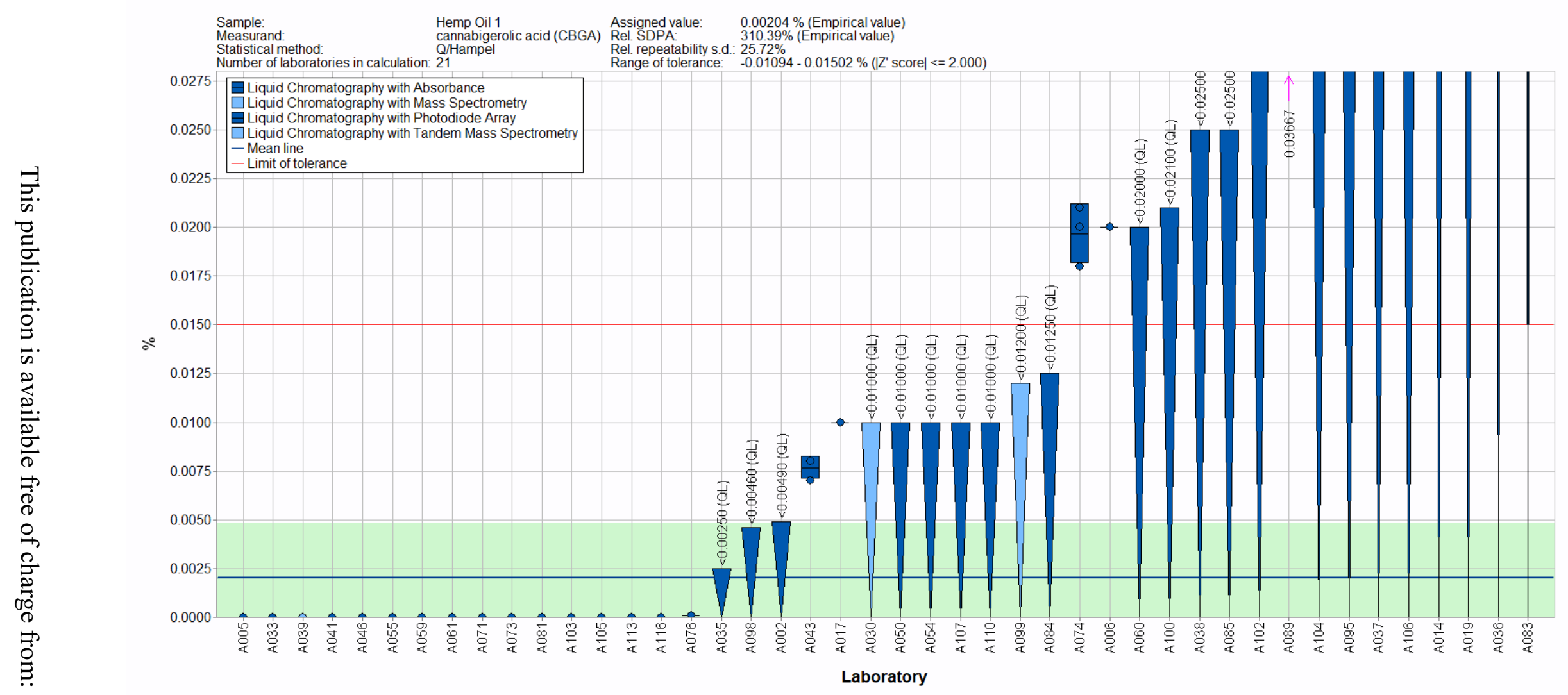

Figure 6-5. CBGA in Hemp Oil 1 (data summary view - analytical method). In this view, individual laboratory data are plotted (circles) with the individual laboratory standard deviation (rectangle). The color of the data point represents the analytical method employed. The solid blue line represents the consensus mean, and the green shaded region represents the $95 \%$ confidence interval for the consensus mean. The solid red line represents the upper consensus range of tolerance, calculated as the values above the consensus mean that results in an acceptable $Z_{\text {comm }}^{\prime}$ score, $\left|Z_{\text {comm }}^{\prime}\right| \leq 2$, with the lower limit set at zero. A NIST value has not been determined in this material. The downward triangle represents data reported as a threshold or LOQ value. 


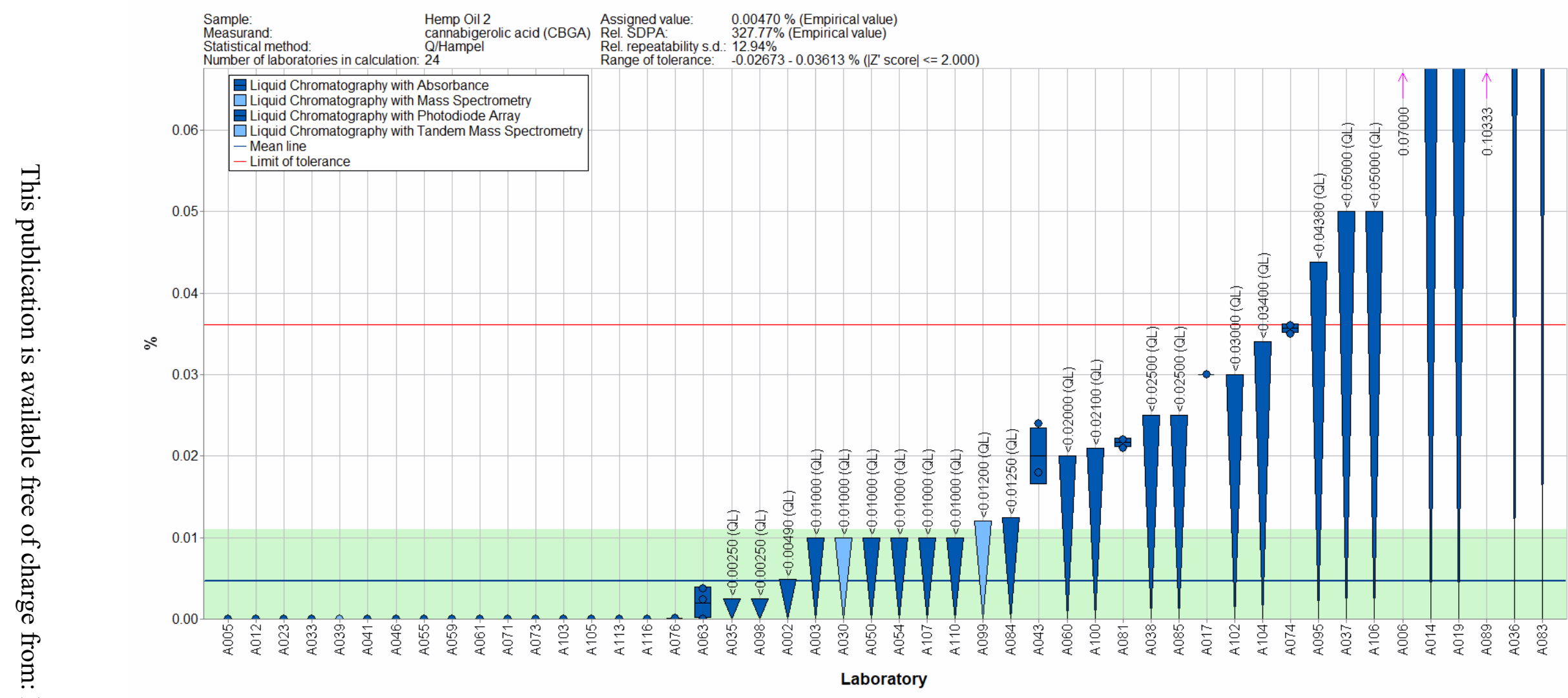

Figure 6-6. CBGA in Hemp Oil 2 (data summary view - analytical method). In this view, individual laboratory data are plotted (circles) with the individual laboratory standard deviation (rectangle). The color of the data point represents the analytical method employed. The solid blue line represents the consensus mean, and the green shaded region represents the $95 \%$ confidence interval for the consensus mean. The solid red line represents the upper consensus range of tolerance, calculated as the values above the consensus mean that results in an acceptable $Z_{\text {comm }}^{\prime}$ score, $\left|Z_{\text {comm }}^{\prime}\right| \leq 2$, with the lower limit set at zero. A NIST value has not been determined in this material. The downward triangle represents data reported as a threshold or LOQ value. 


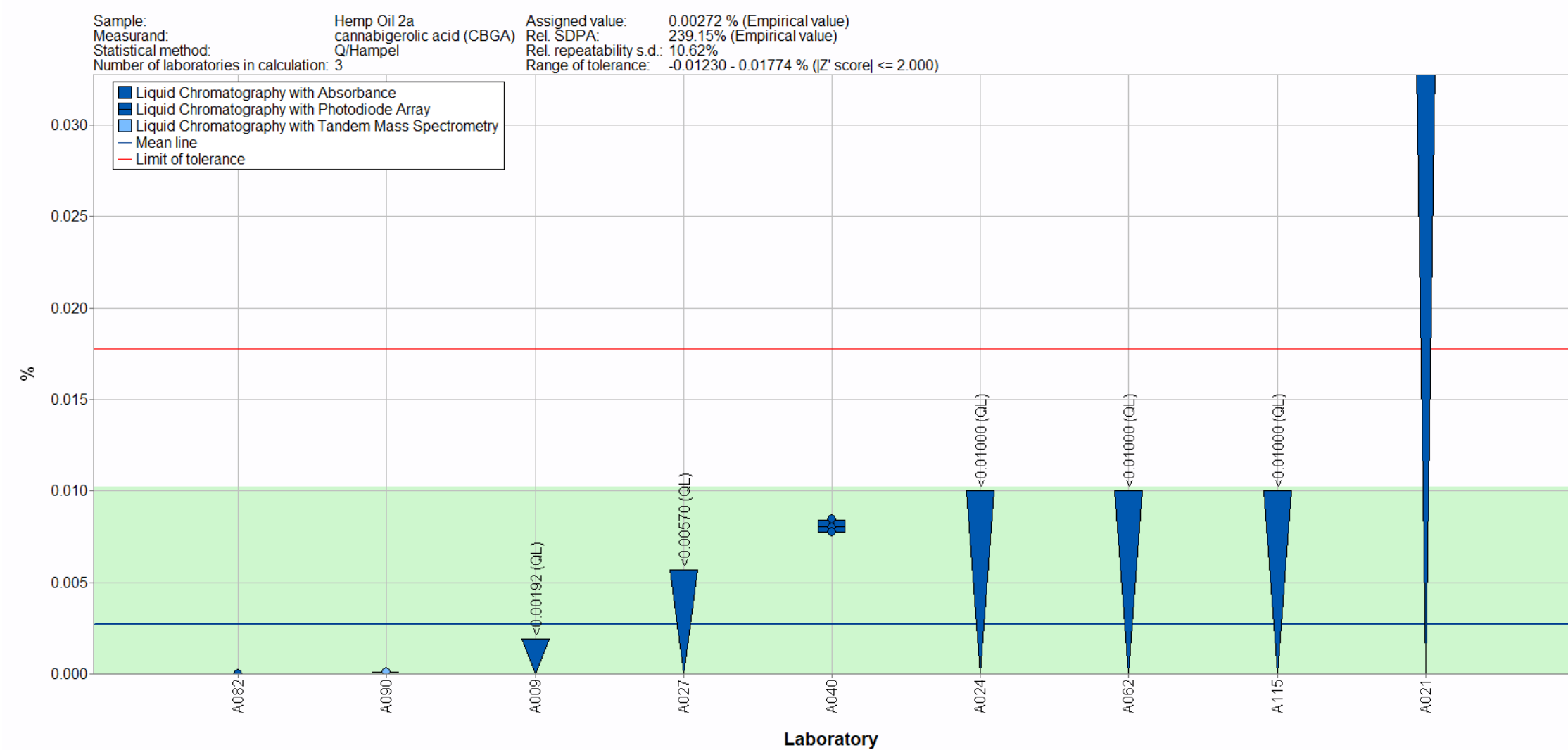

Figure 6-7. CBGA in Hemp Oil 2a (data summary view - analytical method). In this view, individual laboratory data are plotted (circles) with the individual laboratory standard deviation (rectangle). The color of the data point represents the analytical method employed. The solid blue line represents the consensus mean, and the green shaded region represents the $95 \%$ confidence interval for the consensus mean. The solid red line represents the upper consensus range of tolerance, calculated as the values above the consensus mean that results in an acceptable $Z_{\text {comm }}^{\prime}$ score, $\left|Z_{\text {comm }}^{\prime}\right| \leq 2$, with the lower limit set at zero. A NIST value has not been determined in this material. The downward triangle represents data reported as a threshold or LOQ value. 


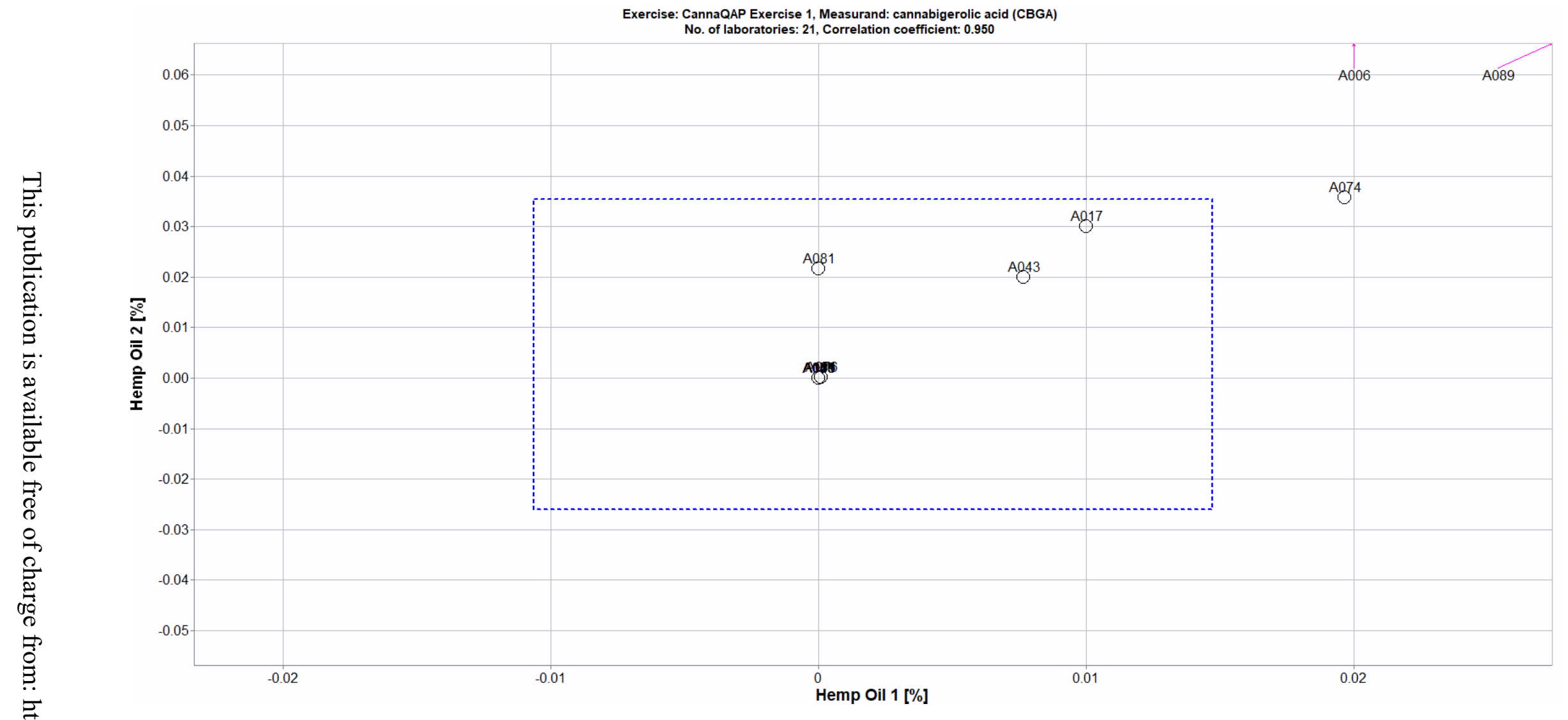

Figure 6-8. Laboratory means for CBGA in Hemp Oil 1 and Hemp Oil 2 (sample/sample comparison view). In this view, the individual laboratory mean for one sample (Hemp Oil 1) is compared to the individual laboratory mean for a second sample (Hemp Oil 2). The dotted blue box represents the consensus range of tolerance for Hemp Oil 1 (x-axis) and Hemp Oil 2 (y-axis), calculated as the values above and below the consensus means that result in an acceptable $Z_{\text {comm }}^{\prime}$ score, $\left|Z_{\text {comm }}^{\prime}\right| \leq 2$. 


\section{SECTION 7: CBL AND CBLA}

Study Overview

CBL is a non-intoxicating cannabinoid often detected at extremely low levels in Cannabis plants and Cannabis-derived products making its determination challenging. ${ }^{11,12}$ As a result, limited information on the potential health benefits of CBL has been conducted and reliable analytical methods are necessary to further scientific research. Unlike other cannabinoids formed through decarboxylation of an acidic precursor, formation of $\mathrm{CBL}$ is understood to occur through the degradation of $\mathrm{CBC}$ during long-term storage or exposure to heat or light. The acidic precursor of CBL, CBLA, has demonstrated resistance to decarboxylation. Participants in this study were asked to use in-house analytical methods to determine the mass fraction (\%) of CBL and CBLA in the three hemp oils. These samples were stored in a control environment that would prevent the degradation of $\mathrm{CBC}$ resulting in extremely low levels of CBL and CBLA.

\section{$\underline{\text { Reporting Statistics }}$}

- The enrollment and reporting statistics for CBL and CBLA are described in the table below for each analyte. Reported values may include non-quantitative results (zero or below LOQ).

\begin{tabular}{|c|c|c|c|c|c|c|}
\hline & \multicolumn{2}{|c|}{$\underline{\text { Hemp Oil } 1}$} & \multicolumn{2}{|c|}{$\underline{\text { Hemp Oil } 2}$} & \multicolumn{2}{|c|}{$\underline{\text { Hemp Oil 2a }}$} \\
\hline & & $\underline{\text { Percent }}$ & & $\underline{\text { Percent }}$ & & $\underline{\text { Percent }}$ \\
\hline & Number of & Reporting & Number of & Reporting & Number of & $\underline{\text { Reporting }}$ \\
\hline$\underline{\text { Analyte }}$ & $\underline{\text { Participants }}$ & $\underline{\text { Results }}$ & $\underline{\text { Participants }}$ & $\underline{\text { Results }}$ & $\underline{\text { Participants }}$ & $\underline{\text { Results }}$ \\
\hline CBL & 32 & $72 \%$ & 34 & $71 \%$ & 19 & $21 \%$ \\
\hline CBLA & 21 & $43 \%$ & 23 & $39 \%$ & 19 & $5 \%$ \\
\hline
\end{tabular}

- Most laboratories reported using solvent extraction or sample dilution for determination of CBL and CBLA in the three hemp oil samples (see table below). Additional sample preparation details are summarized at the end of the report in Appendix I.

\begin{tabular}{ccc} 
Reported Preparation & \multicolumn{2}{c}{ Percent Reporting } \\
Method & $\underline{\text { CBL }}$ & $\underline{\text { CBLA }}$ \\
Solvent Extraction & 63.2 & 65.5 \\
Dilution & 31.6 & 31.0 \\
Other & 0.0 & 0.0 \\
None & 1.8 & 0.0 \\
No Response & 3.5 & 3.4
\end{tabular}

11 Y Wang, B Avula, M ElSohly, M Radwan, M Wang, A Wanas, Z Mehmedic, I Khan. Planta Med 84: 260-266 (2018) https://doi.org/10.1055/s-0043-124873.

12 W Gul, S Gul, M Radwan, A Wanas, Z Mehmedic, I Khan, M Sharaf, M ElSohly. J AOAC Intern 98(6): 15231528 (2015) https://doi.org/10.5740/jaoacint.15-095. 
- Most laboratories reported using LC-PDA or LC-UV for the determination of CBL and CBLA in the three hemp oil samples (see table below). Additional method details are summarized at the end of the report in Appendix I.

\begin{tabular}{ccc} 
Reported Analytical & \multicolumn{2}{c}{ Percent Reporting } \\
Method & $\frac{\text { CBL }}{50.9}$ & CBLA \\
LC-PDA & 35.1 & 79.3 \\
LC-UV & 0.0 & 13.8 \\
LC-MS & 5.3 & 0.0 \\
LC-MS/MS & 0.0 & 0.0 \\
GC-FID & 5.3 & 0.0 \\
GC-MS & 3.5 & 0.0 \\
Other & & 6.9
\end{tabular}

$\underline{\text { Study Results }}$

$C B L$

- No target means or ranges were provided in Table 7-1 for CBL in the three hemp oils.

- The consensus means and ranges for CBL are based on quantitative data from 17 laboratories (Figure 7-1), 19 laboratories (Figure 7-2), and 2 laboratories (Figure 7-3) for Hemp Oil 1, Hemp Oil 2, and Hemp Oil 2a, respectively. Data from participants submitting only one measurement were included in Table 7-2 but were not included in the calculation of consensus statistics. ${ }^{2}$

- A comparison of individual laboratory means for CBL in Hemp Oil 1 and Hemp Oil 2 is summarized in Figure 7-4 for laboratories who reported results for both samples.

CBLA

- No target means or ranges were provided in Table 7-1 for CBLA in the three hemp oils.

- The consensus means and ranges for CBLA are based on quantitative data from 6 laboratories for Hemp Oil 1 (Figure 7-5) and Hemp Oil 2 (Figure 7-6). A consensus mean could not be determined for CBLA in Hemp Oil 2a (Figure 7-7). Data from participants submitting only one measurement were included in Table 7-3 but were not included in the calculation of consensus statistics. ${ }^{2}$

- A comparison of individual laboratory means for CBLA in Hemp Oil 1 and Hemp Oil 2 is summarized in Figure 7-8 for laboratories who reported results for both samples.

Overall

- The between-laboratory variabilities for determination of CBL and CBLA in the hemp oil samples are shown in the table below. 
Between-Laboratory Variability (\% RSD)

\begin{tabular}{|c|c|c|c|}
\hline Analyte & $\underline{\text { Hemp Oil } 1}$ & $\underline{\text { Hemp Oil } 2}$ & Hemp Oil 2a \\
\hline CBL & 11.8 & 9.4 & 24.7 \\
\hline CBLA & 120.2 & 82.5 & NA \\
\hline
\end{tabular}

Study Discussion and Technical Recommendations

The following recommendations are based on results obtained from the participants in this study.

$C B L$

- Approximately $19 \%$ of the laboratories reporting results for CBL provided values outside the consensus ranges for both Hemp Oil 1 and Hemp Oil 2 (Figure 7-4).

- Laboratories reporting results above the consensus mean in Hemp Oil 1 also reported results above the consensus mean for Hemp Oil 2. Trends of this type often indicate a calibration bias.

- Approximately $22 \%$ (5), $21 \%$ (5), and $33 \%$ (1) of the laboratories reported that CBL was present in the Hemp Oil 1, Hemp Oil 2, and Hemp Oil 2a, respectively, at or below their LOQ (non-zero values).

- The between-laboratory variability was higher for CBL in Hemp Oil 2a (24.7 \%) than Hemp Oil $1(11.8 \%)$ and Hemp Oil $2(9.4 \%)$. The variability between individual mean laboratories was higher for CBL in Hemp Oil 1 (5.5 \%) in comparison to Hemp Oil 2 (3.9 \%) and Hemp Oil 2a (2.9\%).

- Hemp Oil 2a was prepared through a methanol/ethanol extraction of Hemp Oil 2 at NIST. The additional processing of Hemp Oil $2 \mathrm{a}$ was expected to result in less variability both within and among participating laboratories.

- The elevated level of variability for Hemp Oil 2a may be an artifact of significantly fewer laboratories reporting results for Hemp Oil 2a (2) compared to Hemp Oil 1 (17) and Hemp Oil 2 (19).

- No additional trends were observed for the sample preparation, analytical methods, and/or analytical parameters reported for $\mathrm{CBL}$ in the three hemp oil samples.

$C B L A$

- Approximately $50 \%$ of all laboratories reported that CBLA was present in the samples at or below their LOQ (non-zero values). The low levels resulted in large consensus ranges and between-laboratory variabilities ( $83 \%$ to $120 \%$ ).

- All laboratories reporting results used LC-UV or LC-PDA methods with only $50 \%$ and $40 \%$ of these laboratories with low enough LOQs to determine CBLA at the consensus level in Hemp Oil 1 and Hemp Oil 2, respectively.

- No additional trends were observed for the sample preparation, analytical methods, and/or analytical parameters reported for CBLA in the three hemp oil samples.

\section{Overall}

- Proper storage conditions are important for hemp oils because CBC can convert to CBL when stored over long periods of time.

- Participants were asked to store the samples under controlled refrigeration $\left(\approx 4{ }^{\circ} \mathrm{C}\right)$. 
- Laboratories should perform in-house evaluations of their storage conditions through stability test of their hemp oils at various environmental factors such as temperature, humidity, and light exposure. ${ }^{5}$

- Over 100 cannabinoids have been identified in Cannabis plant samples with similarities in structure and molecular mass. As a result, chromatographic peak identity should always be confirmed using appropriate reference spectra of pure standards for cannabinoids and is particularly important for cannabinoids that are present at levels close to the method LOQ. Analytical methods should be able to clearly distinguish between cannabinoids.

- The use of appropriate calibration materials and quality assurance samples to establish that a method is in control and being performed correctly may reduce the likelihood of outlying data. Quality assurance samples can be commercially available reference materials (CRMs, SRMs, or RMs) or materials prepared in-house.

- Measurement results should be reported accurately.

- Reported values should be the mass fraction (\%) of the CBL and CBLA in the three hemp oil samples. Participants who reported values based on a volume fraction (\%) for Hemp Oil 2 or Hemp Oil 2a should use the density of the sample for the conversion to mass fraction. Hemp Oil 1 was too viscous to prepare dilutions by volume.

- Zero is not a quantity that can be measured. If values are below LOQ, results should be reported as such. A more appropriate result would be to report that a value is below the LOQ (e.g., " $<0.02 ”)$.

- Laboratories reporting results based on a certain threshold should enter the numerical threshold (e.g., " $<1$ ").

- Laboratories reporting results flagged as outliers should check for calculation errors when preliminary data tables are sent for inspection. One example is to confirm that factors for all dilutions have been properly tabulated or that results are reported in the requested units. 
Table 7-1. Individualized data summary table (NIST) for CBL and CBLA in hemp oils.

National Institute of Standards and Technology

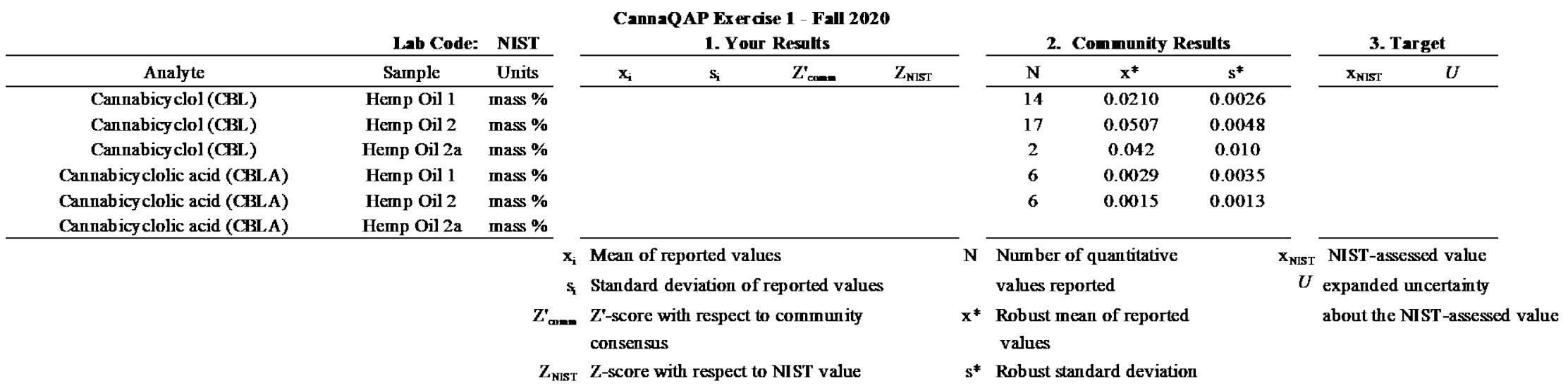


Table 7-2. Data summary table for CBL in hemp oils. Data highlighted in red have been flagged as a data entry of zero or results that include text (e.g., "<LOQ" or "present"). Data highlighted in blue have been identified as outside the consensus tolerance limits and would be estimated to result in an unacceptable $Z_{\text {comm }}^{\prime}$ score, $\left|Z_{\text {comm }}^{\prime}\right| \geq 2$.

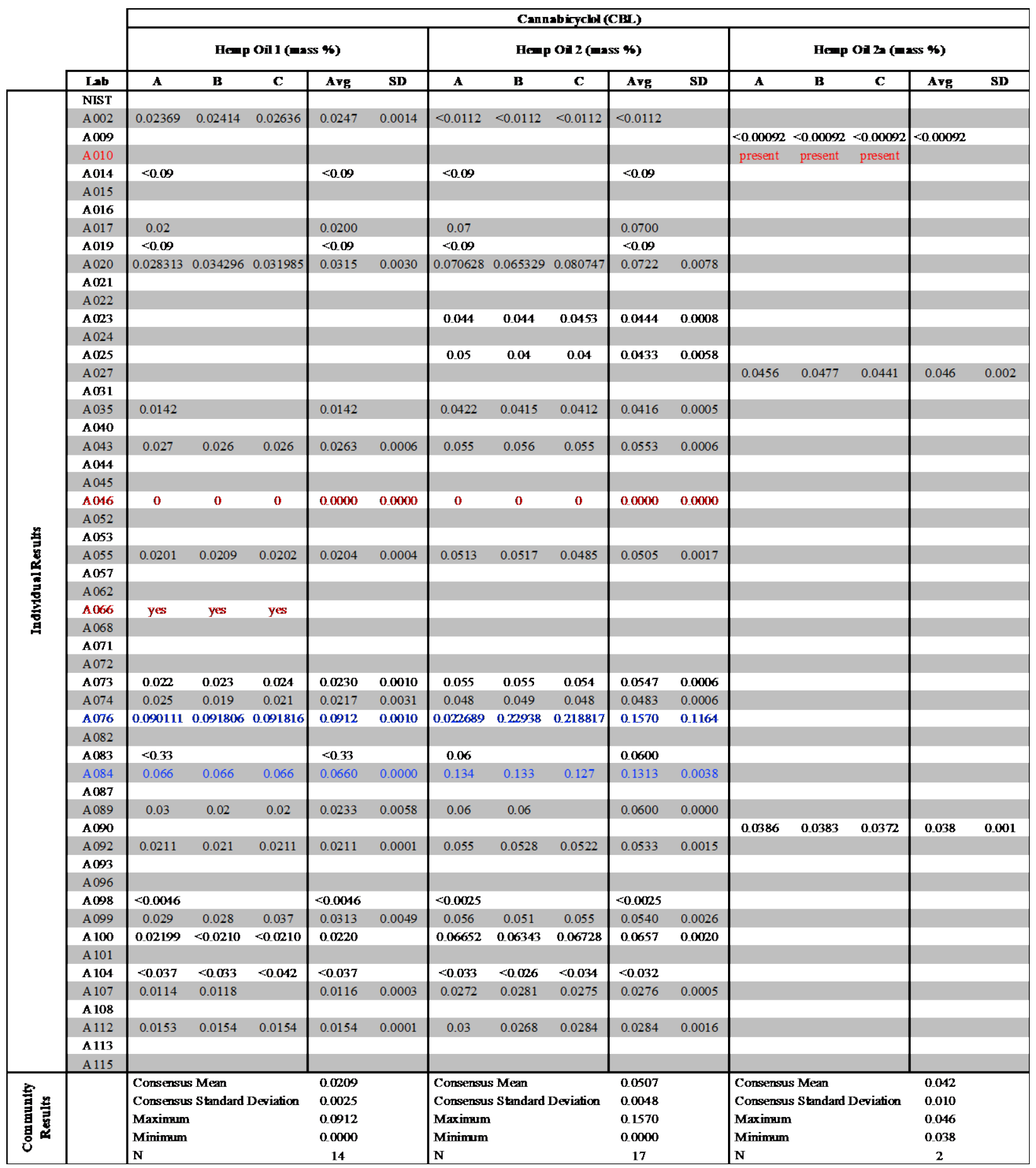




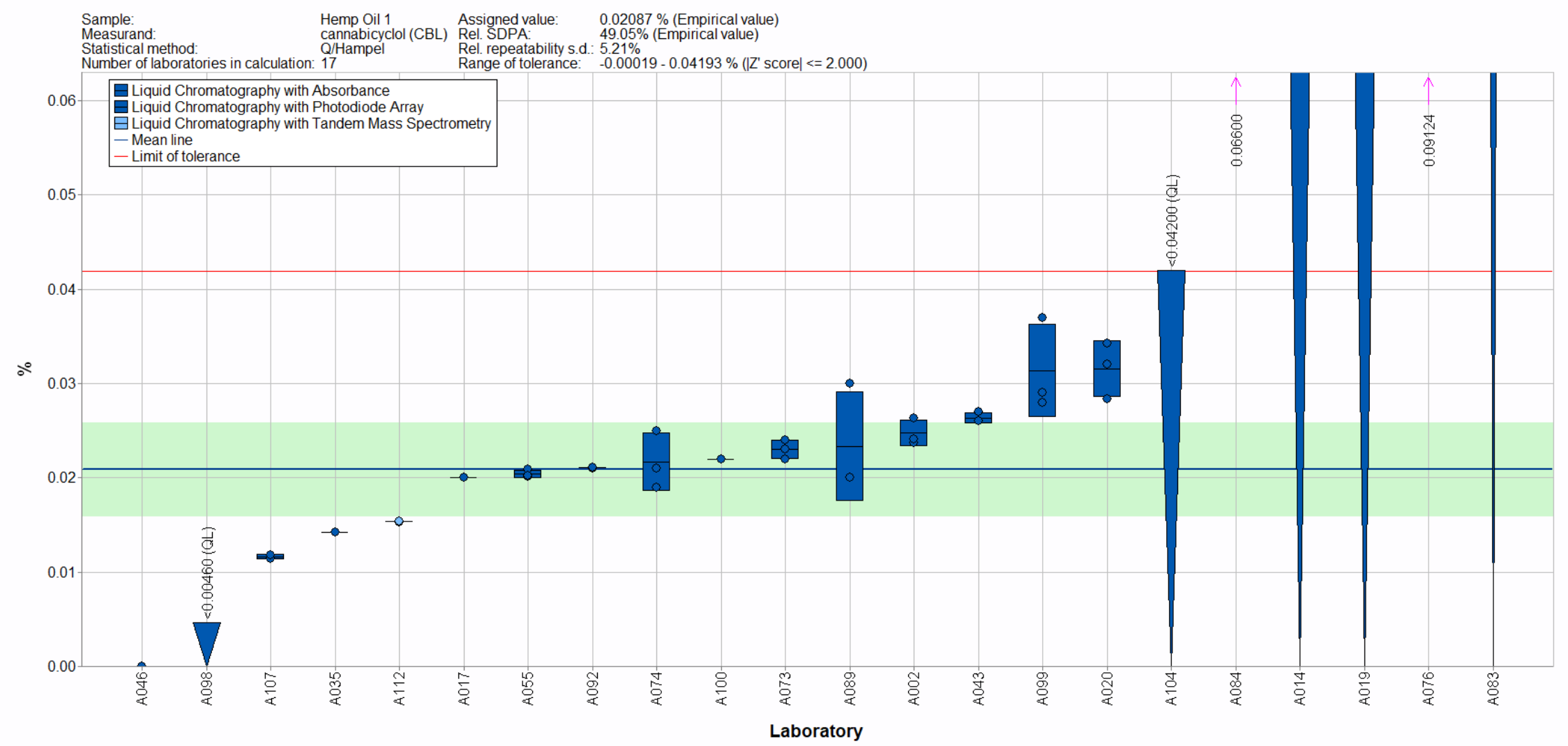

Figure 7-1. CBL in Hemp Oil 1 (data summary view - analytical method). In this view, individual laboratory data are plotted (circles) with the individual laboratory standard deviation (rectangle). The color of the data point represents the analytical method employed. The solid blue line represents the consensus mean, and the green shaded region represents the $95 \%$ confidence interval for the consensus mean. The solid red line represents the upper consensus range of tolerance, calculated as the values above the consensus mean that results in an acceptable $Z_{\text {comm }}^{\prime}$ score, $\left|Z_{\text {comm }}^{\prime}\right| \leq 2$, with the lower limit set at zero. A NIST value has not been determined in this material. The downward triangle represents data reported as a threshold or LOQ value. 


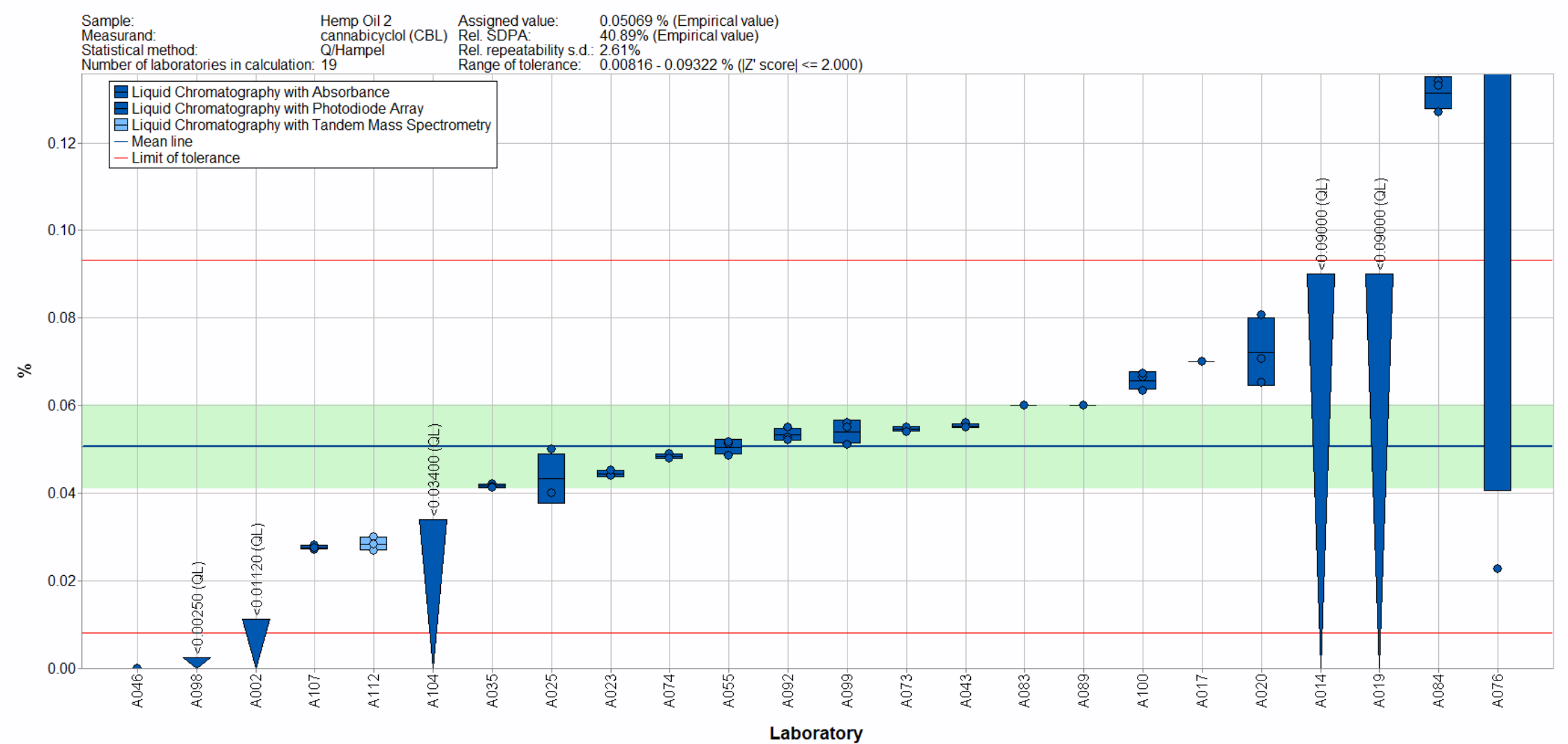

Figure 7-2. CBL in Hemp Oil 2 (data summary view - analytical method). In this view, individual laboratory data are plotted (circles) with the individual laboratory standard deviation (rectangle). The color of the data point represents the analytical method employed. The solid blue line represents the consensus mean, and the green shaded region represents the $95 \%$ confidence interval for the consensus mean. The solid red line represents the upper consensus range of tolerance, calculated as the values above the consensus mean that results in an acceptable $Z_{\text {comm }}^{\prime}$ score, $\left|Z_{\text {comm }}^{\prime}\right| \leq 2$, with the lower limit set at zero. A NIST value has not been determined in this material. The downward triangle represents data reported as a threshold or LOQ value. 


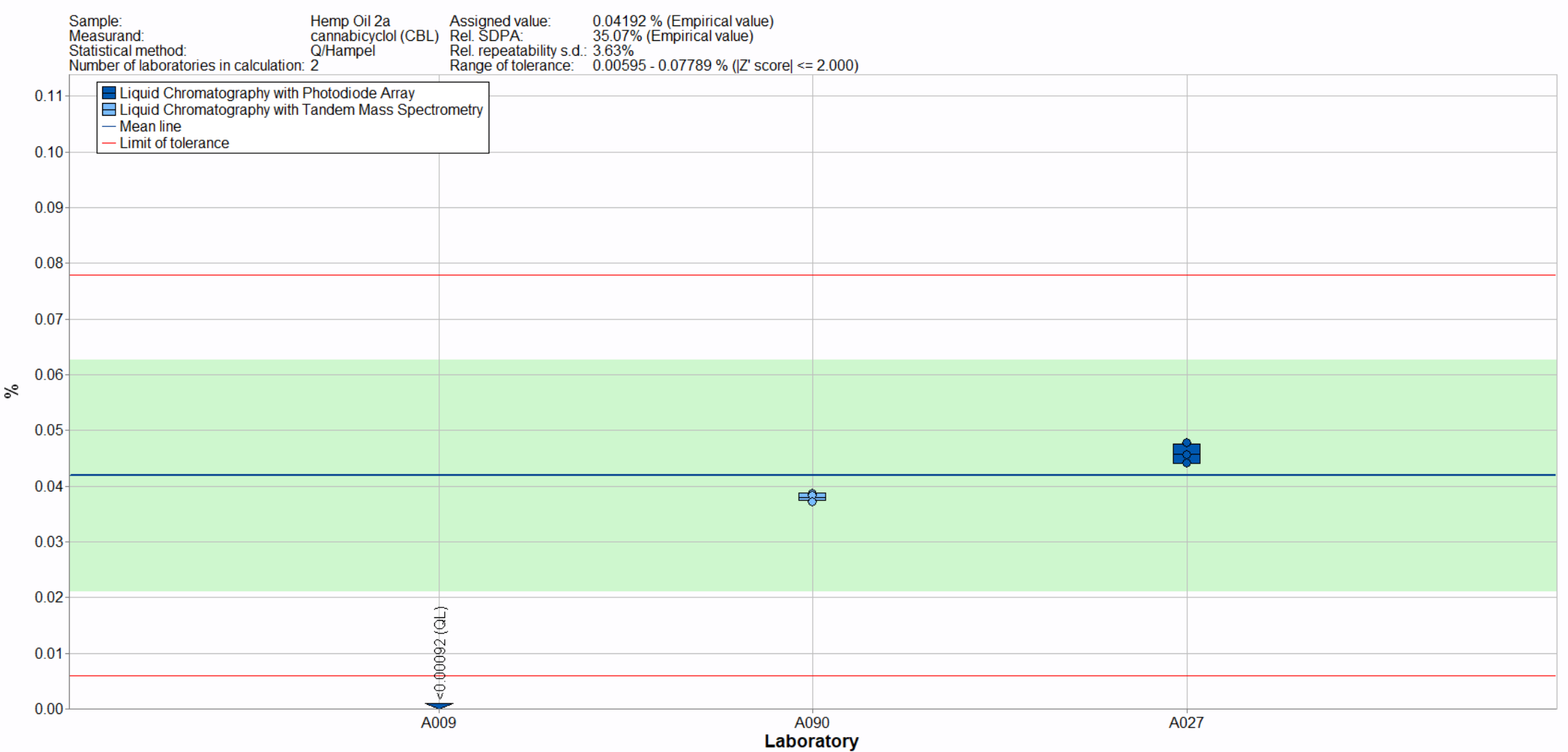

Figure 7-3. CBL in Hemp Oil 2a (data summary view - analytical method). In this view, individual laboratory data are plotted (circles) with the individual laboratory standard deviation (rectangle). The color of the data point represents the analytical method employed. The solid blue line represents the consensus mean, and the green shaded region represents the $95 \%$ confidence interval for the consensus mean. The solid red lines represent the consensus range of tolerance, calculated as the values above and below the consensus mean that result in an acceptable $Z_{\text {comm }}^{\prime}$ score, $\left|Z_{\text {comm }}^{\prime}\right| \leq 2$. A NIST value has not been determined in this material. The downward triangle represents data reported as a threshold or LOQ value. 


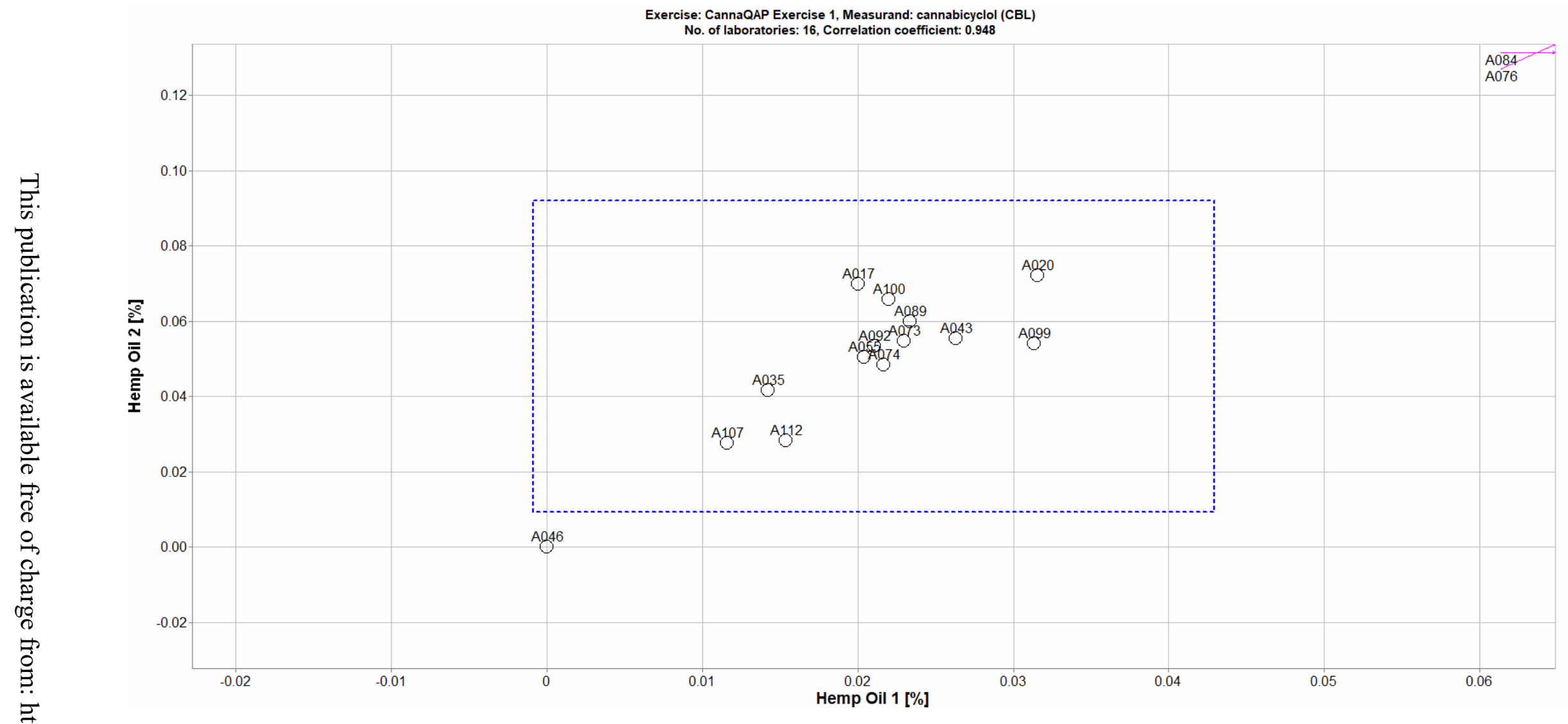

Figure 7-4. Laboratory means for CBL in Hemp Oil 1 and Hemp Oil 2 (sample/sample comparison view). In this view, the individual laboratory mean for one sample (Hemp Oil 1) is compared to the individual laboratory mean for a second sample (Hemp Oil 2). The dotted blue box represents the consensus range of tolerance for Hemp Oil 1 (x-axis) and Hemp Oil 2 (y-axis), calculated as the values above and below the consensus means that result in an acceptable $Z_{\text {comm }}^{\prime}$ score, $\left|Z_{\text {comm }}^{\prime}\right| \leq 2$. 
Table 7-3. Data summary table for CBLA in hemp oils. Data highlighted in red have been flagged as a data entry of zero or results that include text (e.g., "< LOQ" or "present"). Data highlighted in blue have been identified as outside the consensus tolerance limits and would be estimated to result in an unacceptable $Z_{\text {comm }}^{\prime}$ score, $\left|Z_{\text {comm }}^{\prime}\right| \geq 2$. Note: This table spans two pages; the NIST values and consensus values are included on both pages for convenience.

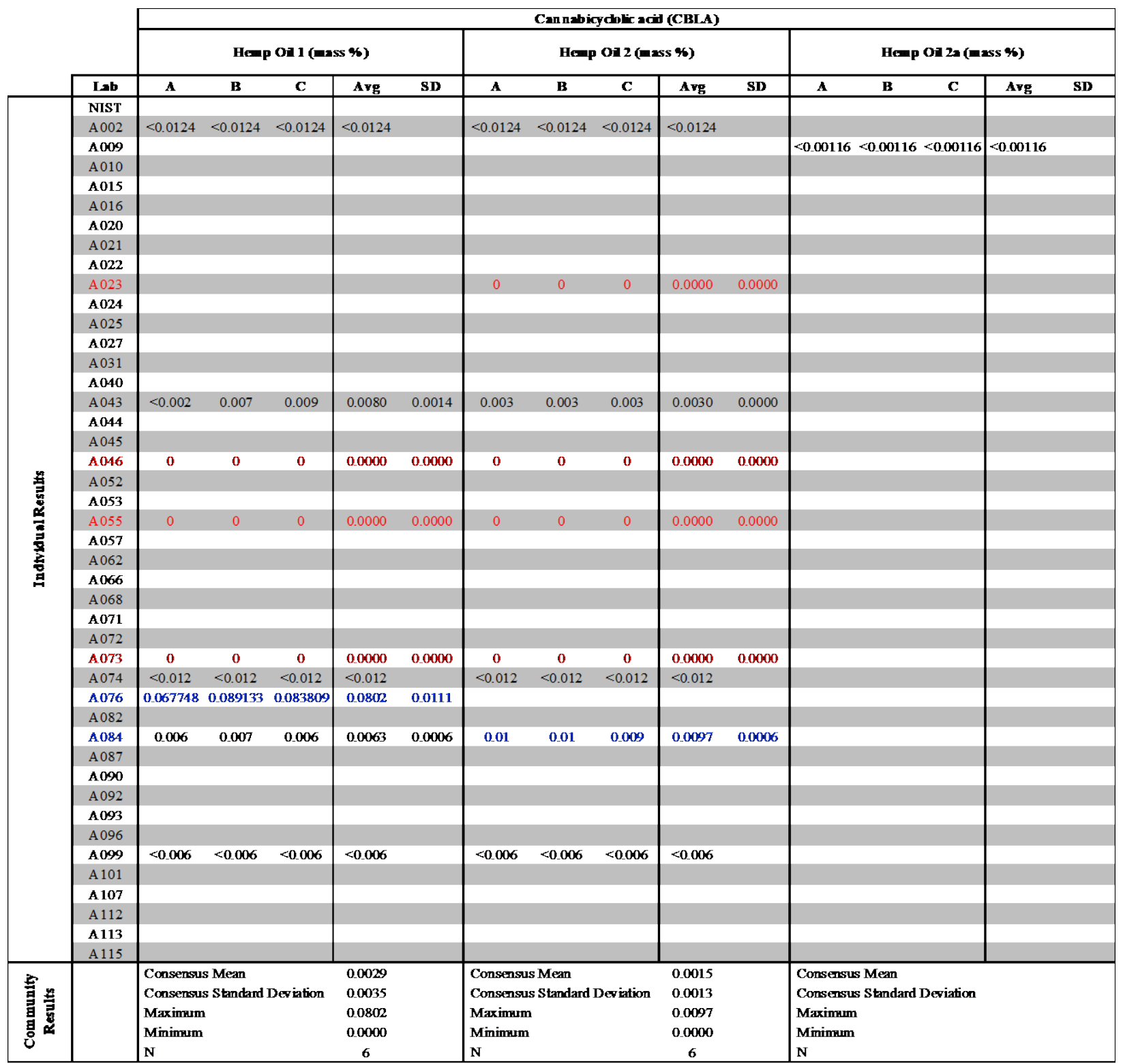




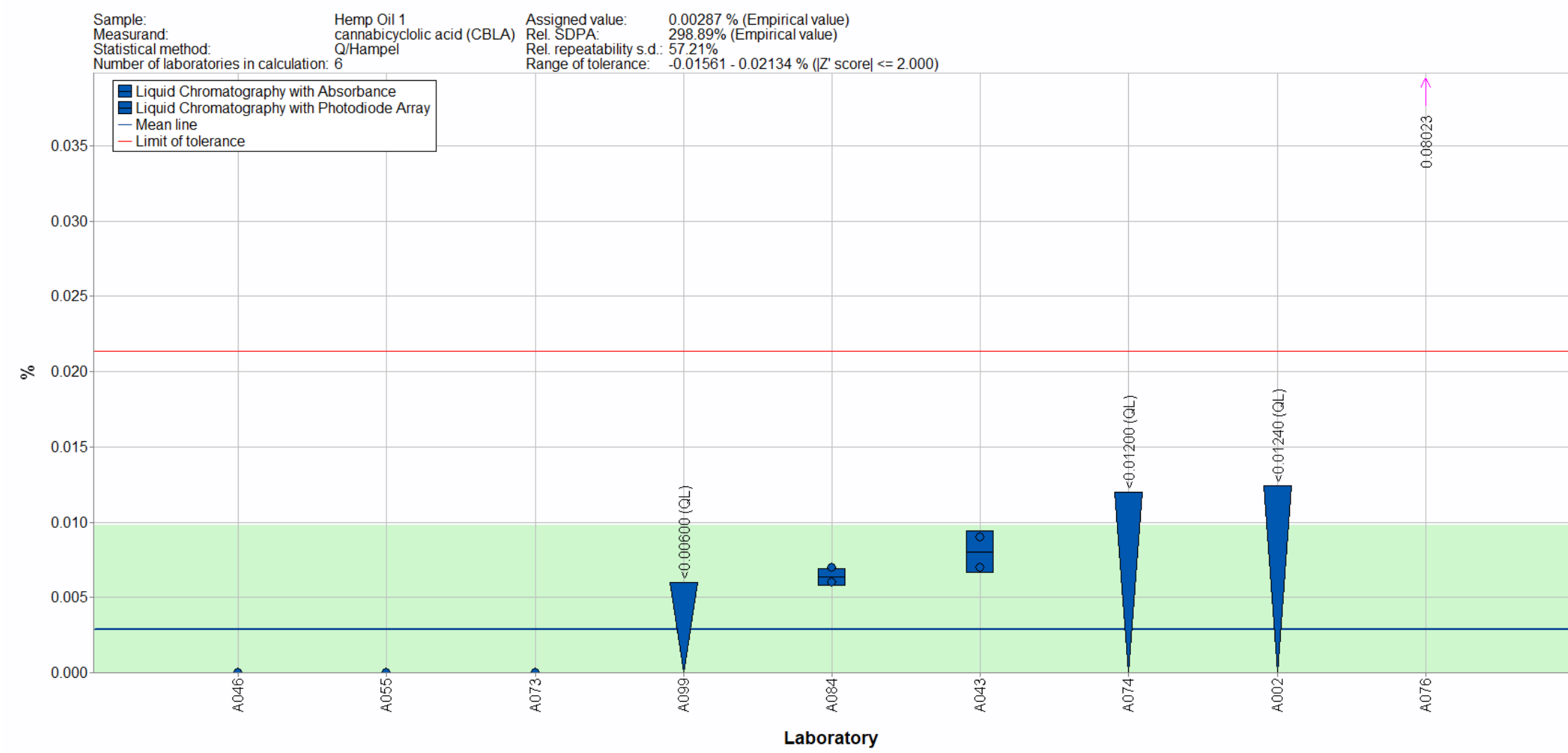

Figure 7-5. CBLA in Hemp Oil 1 (data summary view - analytical method). In this view, individual laboratory data are plotted (circles) with the individual laboratory standard deviation (rectangle). The color of the data point represents the analytical method employed. The solid blue line represents the consensus mean, and the green shaded region represents the $95 \%$ confidence interval for the consensus mean. The solid red line represents the upper consensus range of tolerance, calculated as the values above the consensus mean that results in an acceptable $Z_{\text {comm }}^{\prime}$ score, $\left|Z_{\text {comm }}^{\prime}\right| \leq 2$, with the lower limit set at zero. A NIST value has not been determined in this material. The downward triangle represents data reported as a threshold or LOQ value. 


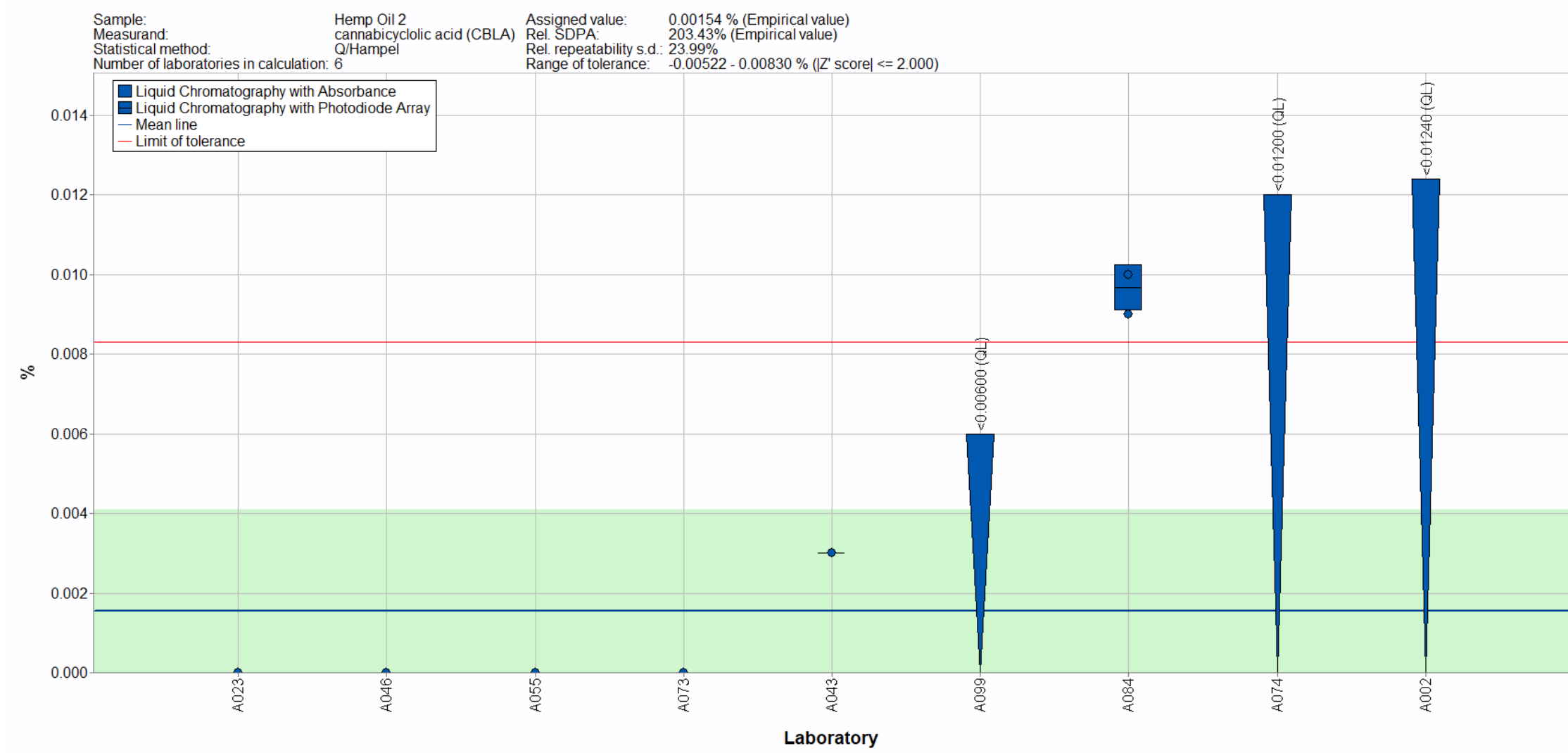

Figure 7-6. CBLA in Hemp Oil 2 (data summary view - analytical method). In this view, individual laboratory data are plotted (circles) with the individual laboratory standard deviation (rectangle). The color of the data point represents the analytical method employed. The solid blue line represents the consensus mean, and the green shaded region represents the $95 \%$ confidence interval for the consensus mean. The solid red line represents the upper consensus range of tolerance, calculated as the values above the consensus mean that results in an acceptable $Z_{\text {comm }}^{\prime}$ score, $\left|Z_{\text {comm }}^{\prime}\right| \leq 2$, with the lower limit set at zero. A NIST value has not been determined in this material. The downward triangle represents data reported as a threshold or LOQ value. 


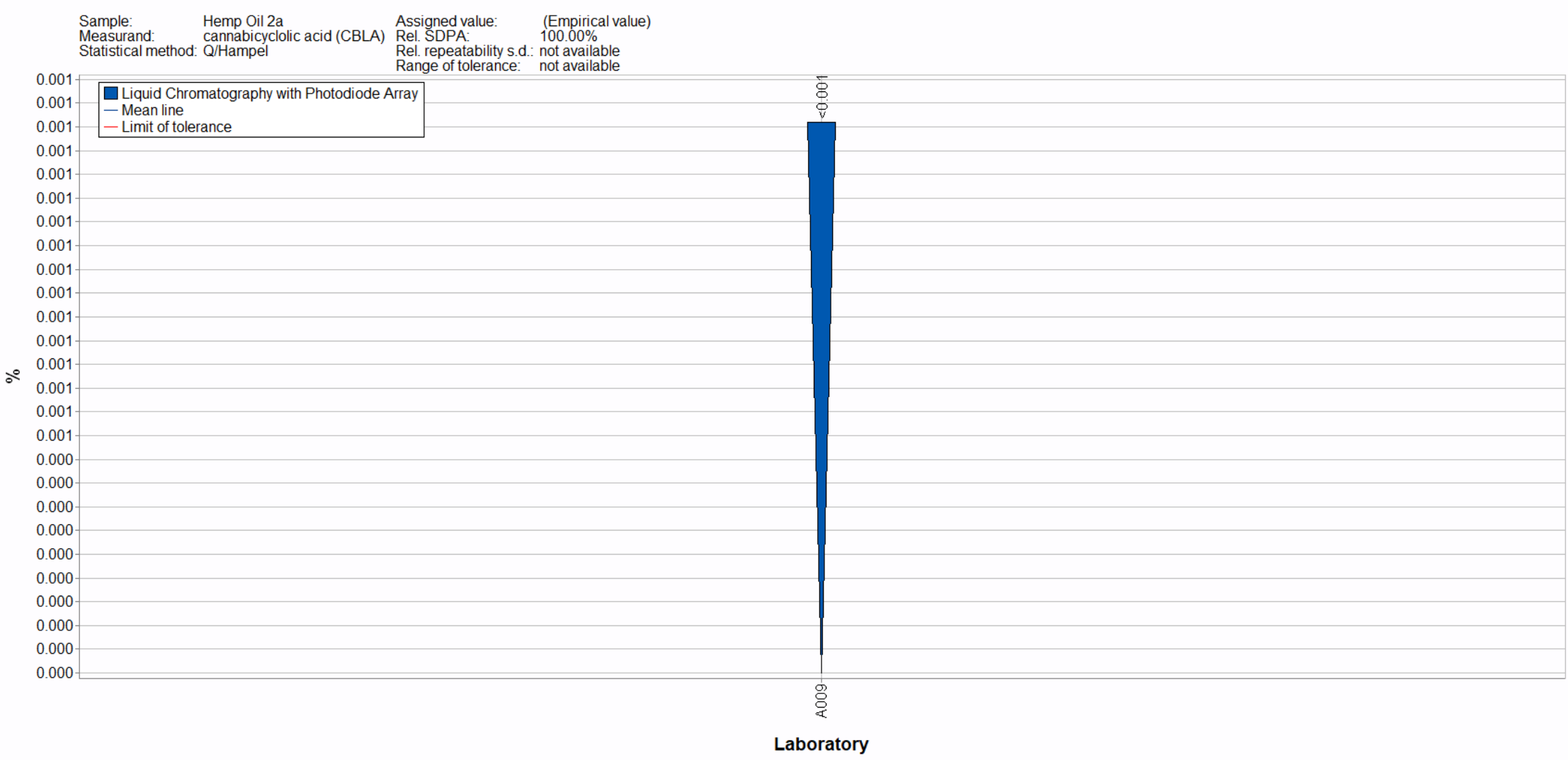

Figure 7-7. CBLA in Hemp Oil 2a (data summary view - analytical method). In this view, individual laboratory data are plotted (circles) with the individual laboratory standard deviation (rectangle). The color of the data point represents the analytical method employed. The downward triangle represents data reported as a threshold or LOQ value. 


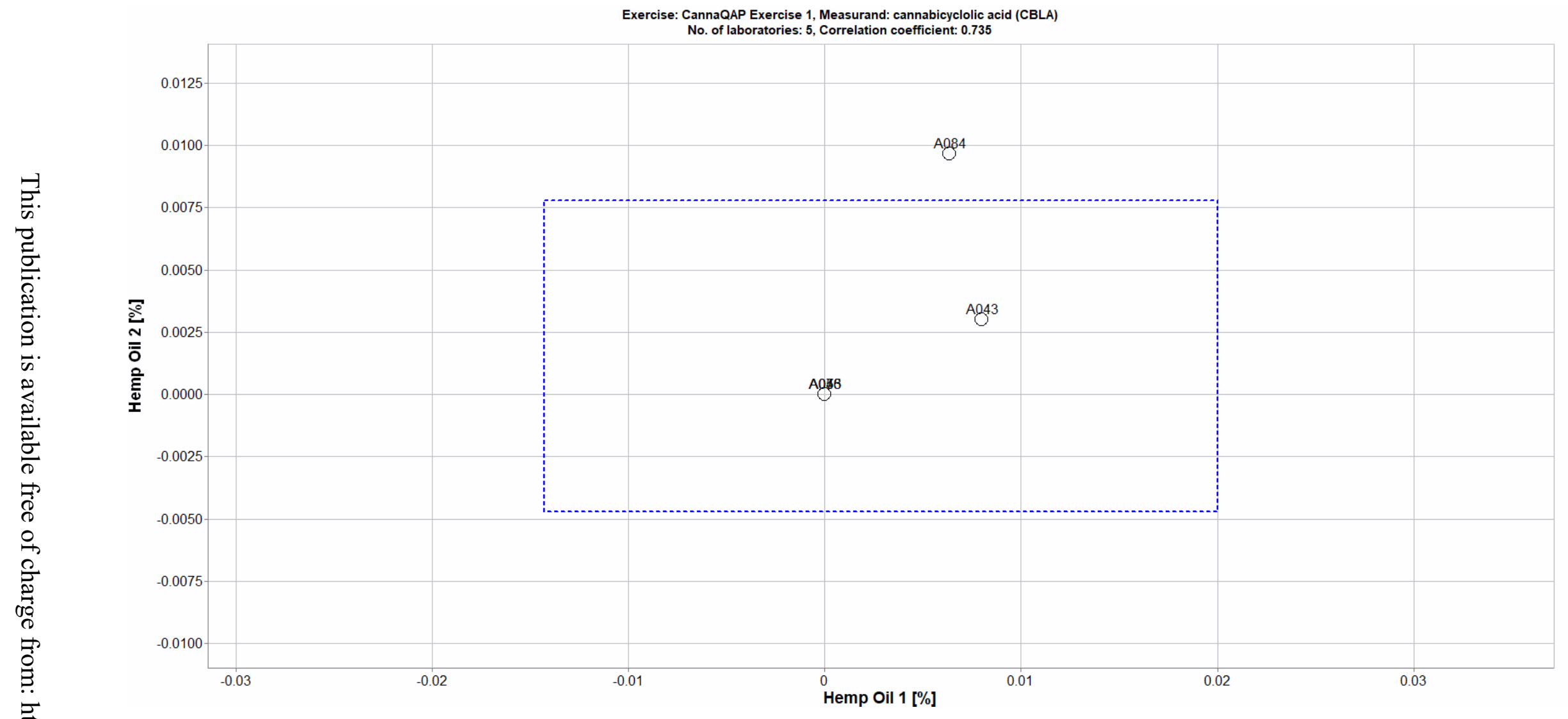

Figure 7-8. Laboratory means for CBLA in Hemp Oil 1 and Hemp Oil 2 (sample/sample comparison view). In this view, the individual laboratory mean for one sample (Hemp Oil 1) is compared to the individual laboratory mean for a second sample (Hemp Oil 2). The dotted blue box represents the consensus range of tolerance for Hemp Oil 1 (x-axis) and Hemp Oil 2 (y-axis), calculated as the values above and below the consensus means that result in an acceptable $Z_{\text {comm }}^{\prime}$ score, $\left|Z_{\text {comm }}^{\prime}\right| \leq 2$. 


\section{SECTION 8: CBN AND CBNA}

Study Overview

CBN is a non-intoxicating cannabinoid often detected in Cannabis plants and Cannabis-derived products at low levels and has attracted interest due to research showing potential health benefits. ${ }^{9}$ CBN and its acidic precursor CBNA are formed through the oxidation of $\Delta^{9}$-THC and THCA, respectively, in Cannabis plant following exposure to prolonged periods of light or heat requiring reliable analytical methods. Participants in this study were asked to use in-house analytical methods to determine the mass fraction (\%) of CBN and CBNA in the three hemp oils. The preparation of these hemp oils included a decarboxylation step resulting in extremely low levels of CBNA and levels of CBN consistent with normal ranges in commercial products.

\section{$\underline{\text { Reporting Statistics }}$}

- The enrollment and reporting statistics for CBN and CBNA are described in the table below for each analyte. Reported values may include non-quantitative results (zero or below LOQ).

\begin{tabular}{|c|c|c|c|c|c|c|}
\hline & \multicolumn{2}{|c|}{$\underline{\text { Hemp Oil } 1}$} & \multicolumn{2}{|c|}{ Hemp Oil 2} & \multicolumn{2}{|c|}{ Hemp Oil 2a } \\
\hline & & Percent & & Percent & & Percent \\
\hline & $\underline{\text { Number of }}$ & $\underline{\text { Reporting }}$ & $\underline{\text { Number of }}$ & $\underline{\text { Reporting }}$ & $\underline{\text { Number of }}$ & $\underline{\text { Reporting }}$ \\
\hline Analyte & $\underline{\text { Participants }}$ & $\underline{\text { Results }}$ & $\underline{\text { Participants }}$ & $\underline{\text { Results }}$ & $\underline{\text { Participants }}$ & $\underline{\text { Results }}$ \\
\hline $\mathrm{CBN}$ & 75 & $81 \%$ & 85 & $80 \%$ & 19 & $63 \%$ \\
\hline CBNA & 29 & $55 \%$ & 31 & $48 \%$ & 19 & $11 \%$ \\
\hline
\end{tabular}

- Most laboratories reported using solvent extraction or sample dilution for determination of CBN and CBNA in the three hemp oil samples (see table below). Additional sample preparation details are summarized at the end of the report in Appendix I.

\begin{tabular}{|c|c|c|}
\hline Reported & \multicolumn{2}{|c|}{ Percent Reporting } \\
\hline Preparation Method & CBN & CBNA \\
\hline Solvent Extraction & 68.5 & 69.6 \\
\hline Dilution & 25.5 & 26.1 \\
\hline Other & 2.0 & 2.2 \\
\hline None & 2.0 & 2.2 \\
\hline No Response & 4.0 & 2.2 \\
\hline
\end{tabular}


- Most laboratories reported using LC-UV or LC-PDA for the determination of CBN and CBNA (see table below). Additional method details are summarized at the end of the report in Appendix I.

\begin{tabular}{|c|c|c|}
\hline Reported & \multicolumn{2}{|c|}{ Percent Reporting } \\
\hline Analytical Method & $\underline{\mathrm{CBN}}$ & $\underline{\mathrm{CBNA}}$ \\
\hline LC-PDA & 61.7 & 52.2 \\
\hline LC-UV & 28.2 & 43.5 \\
\hline LC-MS & 1.3 & 0.0 \\
\hline LC-MS/MS & 3.4 & 0.0 \\
\hline GC-FID & 2.7 & 0.0 \\
\hline GC-MS & 2.0 & 0.0 \\
\hline Other & 0.7 & 4.3 \\
\hline
\end{tabular}

$\underline{\text { Study Results }}$

$C B N$

- The mass fractions (\%) for CBN in the hemp oil samples were determined by NIST using LCPDA as described in Section 1 and are summarized in Table 8-1. These NIST values are used as the target means and ranges summarized in Table 8-2 for comparison to the participant results.

- The target and consensus means and ranges are summarized for $\mathrm{CBN}$ via different analytical methods in Figure 8-1, Figure 8-2, and Figure 8-3, which include data from laboratories submitting two or three results for CBN. Data from participants submitting only one measurement were included in Table 8-2 but were not included in the calculation of consensus statistics. ${ }^{2}$

- For CBN in Hemp Oil 1, the consensus range was based on quantitative results from 49 laboratories and overlaps approximately $90 \%$ of the target range (Figure 8-1).

- The individual laboratory means from 33 laboratories ( $81 \%$ of those reporting results) were outside the NIST range of tolerance for CBN in Hemp Oil 1.

- The individual laboratory means from 11 laboratories (27\% of those reporting results) were outside the acceptable $Z_{\text {comm }}^{\prime}$ score for CBN in Hemp Oil 1.

- The thresholds or LOQs for 15 of 19 laboratories reporting qualitative results were below the target mean for CBN in Hemp Oil 1.

- For CBN in Hemp Oil 2, the consensus range was based on quantitative results from 49 laboratories and overlaps approximately $35 \%$ of the target range (Figure 8-2).

- The individual laboratory means from 17 laboratories (35\% of those reporting results) were outside the NIST range of tolerance for CBN in Hemp Oil 2.

- The individual laboratory means from 9 laboratories (18\% of those reporting results) were outside the acceptable $Z_{\text {comm }}^{\prime}$ score for CBN in Hemp Oil 2.

- The thresholds or LOQs for 11 of 16 laboratories reporting qualitative results were below the target mean for CBN in Hemp Oil 2.

- For CBN in Hemp Oil 2a, the consensus range was based on quantitative results from 10 laboratories and overlaps approximately $55 \%$ of the target range (Figure 8-3). 
- The individual laboratory means from 5 laboratories (50\% of those reporting results) were outside the NIST range of tolerance for CBN in Hemp Oil 2a.

- All laboratory means were within the acceptable $Z_{\text {comm }}^{\prime}$ score for CBN in Hemp Oil $2 a$.

- The single laboratory reporting a qualitative threshold or LOQ for CBN in Hemp Oil 2 a was above the target mean.

- A comparison of individual laboratory means for CBN in Hemp Oil 1 and Hemp Oil 2 is summarized in Figure 8-4 for laboratories who reported results for both samples.

CBNA

- No target means or ranges were provided in Table 8-1 for CBNA in the three hemp oils.

- The consensus means and ranges for CBDVA are based on quantitative data from 7 laboratories for Hemp Oil 1 (Figure 8-5) and 7 laboratories for Hemp Oil 2 (Figure 8-6), respectively. A consensus mean could not be determined in Hemp Oil 2a (Figure 8-7). Data from participants submitting only one measurement were included in Table 8-3 but were not included in the calculation of consensus statistics. ${ }^{2}$

- A comparison of individual laboratory means for CBNA in Hemp Oil 1 and Hemp Oil 2 is summarized in Figure 8-8 for laboratories who reported results for both samples.

Overall

- The between-laboratory variabilities for determination of CBN and CBNA in the hemp oil samples are shown in the table below.

Between-Laboratory Variability (\% RSD)

\begin{tabular}{|c|c|c|c|}
\hline Analyte & Hemp Oil 1 & Hemp Oil 2 & Hemp Oil 2a \\
\hline $\mathrm{CBN}$ & 6.8 & 5.1 & 9.3 \\
\hline CBNA & 81.4 & 60.2 & NA \\
\hline
\end{tabular}

Study Discussion and Technical Recommendations

The following recommendations are based on results obtained from the participants in this study.

$C B N$

- Approximately $30 \%$ of the laboratories reporting results for $\mathrm{CBN}$ provided values outside the consensus ranges for both Hemp Oil 1 and Hemp Oil 2 (Figure 8-4).

- Laboratories reporting results below the consensus mean in Hemp Oil 1 also reported results below the consensus mean for Hemp Oil 2. The reverse is also observed for laboratories reporting values above the consensus mean in both samples. Trends of this type often indicate a calibration bias.

- The between-laboratory variability was higher for CBN in Hemp Oil 2a (9.3\%) than Hemp Oil $1(6.8 \%)$ and Hemp Oil $2(5.1 \%)$. The variability between individual mean laboratories were relatively close for CBN in Hemp Oil 1 (5.9\%), Hemp Oil 2 (5.9\%), and Hemp Oil 2a $(5.2 \%)$. 
- Hemp Oil 2a was prepared through a methanol/ethanol extraction of Hemp Oil 2 at NIST. The additional processing of Hemp Oil 2a was expected to result in minimal variability both within and among participating laboratories.

- The elevated level of variability may be an artifact of significantly fewer laboratories reporting results for Hemp Oil 2a (10) compared to Hemp Oil 1 (49) and Hemp Oil 2 (49).

- No additional trends were observed for the sample preparation, analytical methods, and/or analytical parameters reported for $\mathrm{CBN}$ in the three hemp oil samples.

CBNA

- Approximately $20 \%$ of the laboratories reporting results for CBNA provided values outside the consensus ranges for both Hemp Oil 1 and Hemp Oil 2 (Figure 8-8).

- Laboratories reporting results above the consensus mean in Hemp Oil 1 also reported results above the consensus mean for Hemp Oil 2. Trends of this type often indicate a calibration bias.

- Most laboratories reported that CBNA was present in the samples at or below their LOQ (nonzero values). The low levels resulted in large consensus ranges and between-laboratory variabilities $(60 \%$ to $81 \%)$.

- Approximately $96 \%$ of the laboratories reporting results used LC-UV or LC-PDA methods with only $31 \%$ and $20 \%$ of these laboratories with low enough LOQs to determine CBNA at the consensus level in Hemp Oil 1 and Hemp Oil 2, respectively.

- No additional trends were observed for the sample preparation, analytical methods, and/or analytical parameters reported for CBNA in the three hemp oil samples.

\section{Overall}

- Proper storage conditions are important for hemp oils because CBNA can readily convert to $\mathrm{CBN}$ when stored at elevated or room temperatures.

- Participants were asked to store the samples under controlled refrigeration $\left(\approx 4{ }^{\circ} \mathrm{C}\right)$.

- Laboratories should perform in-house evaluations of their storage conditions through stability test of their hemp oils at various environmental factors such as temperature, humidity, and light exposure. ${ }^{5}$

- Over 100 cannabinoids have been identified in Cannabis plant samples with similarities in structure and molecular mass. As a result, chromatographic peak identity should always be confirmed using appropriate reference spectra of pure standards for cannabinoids and is particularly important for cannabinoids that are present at levels close to the method LOQ. Analytical methods should be able to clearly distinguish between cannabinoids.

- The use of appropriate calibration materials and quality assurance samples to establish that a method is in control and being performed correctly may reduce the likelihood of outlying data. Quality assurance samples can be commercially available reference materials (CRMs, SRMs, or RMs) or materials prepared in-house.

- Measurement results should be reported accurately.

- Reported values should be the mass fraction (\%) of the CBN and CBNA in the three hemp oil samples. Participants who reported values based on a volume fraction (\%) for Hemp Oil 2 or Hemp Oil 2a should use the density of the sample for the conversion to mass fraction. Hemp Oil 1 was too viscous to prepare dilutions by volume. 
- Zero is not a quantity that can be measured. If values are below LOQ, results should be reported as such. A more appropriate result would be to report that a value is below the LOQ (e.g., " < 0.02 ”).

- Laboratories reporting results based on a certain threshold should enter the numerical threshold (e.g., "< 1").

- Laboratories reporting results flagged as outliers should check for calculation errors when preliminary data tables are sent for inspection. One example is to confirm that factors for all dilutions have been properly tabulated or that results are reported in the requested units. 
Table 8-1. Individualized data summary table (NIST) for CBN and CBNA in hemp oils.

\section{National Institute of Standards and Technology}

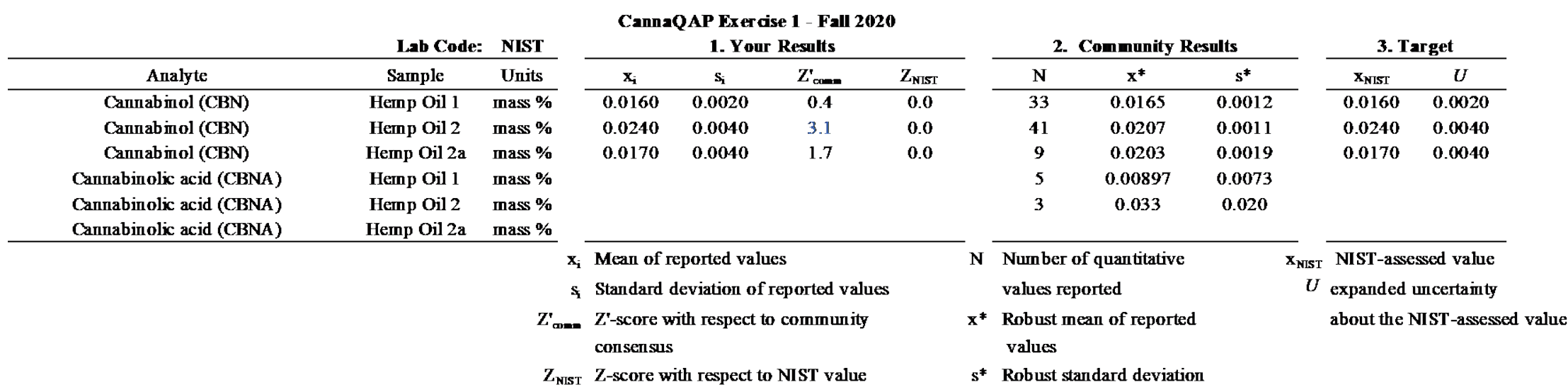


Table 8-2. Data summary table for $\mathrm{CBN}$ in hemp oils. Data highlighted in red have been flagged as a data entry of zero or results that include text (e.g., "<LOQ" or "present"). Data highlighted in blue have been identified as outside the consensus tolerance limits and would be estimated to result in an unacceptable $Z_{\text {comm }}^{\prime}$ score, $\left|Z_{\text {comm }}^{\prime}\right| \geq 2$. Note: This table spans two pages; the NIST values and consensus values are included on both pages for convenience.

\begin{tabular}{|c|c|c|c|c|c|c|c|c|c|c|c|c|c|c|c|c|}
\hline & \multirow[b]{3}{*}{ Lab } & \multicolumn{15}{|c|}{ Cannabingl (CEN) } \\
\hline & & \multicolumn{5}{|c|}{ He=p Oill (mass \%) } & \multicolumn{5}{|c|}{ Heap OAl 2 (mass \%) } & \multicolumn{5}{|c|}{ Henp OI 2a (mass \%) } \\
\hline & & $\mathbf{A}$ & $\mathbf{B}$ & $\mathbf{C}$ & Avg & SD & $\mathbf{A}$ & $\mathbf{B}$ & $\mathbf{C}$ & Avg & SD & $\mathbf{A}$ & $\mathbf{B}$ & $\mathbf{C}$ & Avg & SD \\
\hline \multirow{50}{*}{ 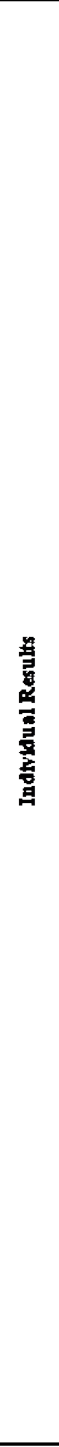 } & NIST & & & & 0.0160 & 0.0020 & & & & 0.0240 & 0.0040 & & & & 0.0170 & $0 \_0040$ \\
\hline & A 001 & $<0.05$ & $<0.05$ & $<0.05$ & $<0.05$ & & $<0.15$ & $<0.15$ & $<0.15$ & $<0.15$ & & & & & & \\
\hline & A002 & 0.02259 & 0.02545 & 0.02212 & 0.0234 & 0.0018 & 0.026719 & 0.024345 & 0.026389 & 0.0258 & 0.0013 & & & & & \\
\hline & A 003 & & & & & & $<0.05$ & $<0.05$ & $<0.05$ & $<0.05$ & & & & & & \\
\hline & A004 & 0.02 & 0.02 & 0.02 & 0.0200 & 0.0000 & 0.03 & 0.03 & 0.02 & 0.0267 & 0.0058 & & & & & \\
\hline & A 005 & 0.0176 & 0.0174 & 0.0188 & 0.0179 & 0.0008 & 0.0267 & 0.0264 & 0.0264 & 0.0265 & 0.0002 & & & & & \\
\hline & A 006 & 0.08 & & & 0.0800 & & 0.03 & & & 0.0300 & & & & & & \\
\hline & A 007 & & & & & & & & & & & & & & & \\
\hline & A008 & 0.011 & & & 0.0110 & & & & & & & & & & & \\
\hline & A 009 & & & & & & & & & & & 0.012 & 0.012 & 0.013 & 0.0123 & 0.0006 \\
\hline & A010 & & & & & & & & & & & present & present & present & & \\
\hline & A 011 & & & & & & & & & & & & & & & \\
\hline & A012 & & & & & & 0.01275 & 0.01285 & 0.01293 & 0.0128 & 0.0001 & & & & & \\
\hline & A 013 & 0.019 & 0.02 & 0.02 & 0.0197 & 0.0006 & 0.023 & 0.026 & 0.026 & 0.0250 & 0.0017 & & & & & \\
\hline & A014 & $<0.09$ & & & $<0.09$ & & $<0.09$ & & & $<0.09$ & & & & & & \\
\hline & A 015 & & & & & & & & & & & & 0.02 & 0.03 & 0.0250 & 0.0071 \\
\hline & A016 & & & & & & & & & & & & & & & \\
\hline & A 017 & 0.02 & & & 0.0200 & & 0.02 & & & 0.0200 & & & & & & \\
\hline & A018 & & & & & & & & & & & & & & & \\
\hline & A 019 & $<0.09$ & & & $<0.09$ & & $<0.09$ & & & $<0.09$ & & & & & & \\
\hline & A020 & 0.020738 & 0.020595 & 0.02041 & 0.0206 & 0.0002 & 0.025181 & 0.024386 & 0.027899 & 0.0258 & 0.0018 & & & & & \\
\hline & A 021 & & & & & & & & & & & $<0.05$ & $<0.05$ & $<0.05$ & $<0.05$ & \\
\hline & A022 & & & & & & & & & & & 0.0169 & 0.0171 & 0.0168 & 0.0169 & 0.0002 \\
\hline & $\mathrm{A} 023$ & & & & & & 0.0235 & 0.0245 & 0.0239 & 0.0240 & 0.0005 & & & & & \\
\hline & A024 & & & & & & & & & & & 0.022 & 0.024 & 0.025 & 0.0237 & 0.0015 \\
\hline & A 025 & & & & & & 0.03 & 0.03 & 0.02 & 0.0267 & 0.0058 & & & & & \\
\hline & A026 & & & & & & & & & & & & & & & \\
\hline & A 027 & & & & & & & & & & & 0.0288 & 0.0287 & 0.0291 & 0.0289 & 0.0002 \\
\hline & A028 & $<0.0630$ & $<0.0630$ & $<0.0630$ & $<0.0630$ & & $<0.0630$ & $<0.0630$ & $<0.0630$ & $<0.0630$ & & & & & & \\
\hline & A 030 & 0.033 & & & 0.0330 & & 0.023 & & & 0.0230 & & & & & & \\
\hline & A031 & $<0.05$ & $<0.05$ & $<0.05$ & $<0.05$ & & $<0.15$ & $<0.15$ & $<0.15$ & $<0.15$ & & & & & & \\
\hline & A 033 & 0.02 & 0.02 & 0.02 & 0.0200 & 0.0000 & 0.02 & 0.02 & 0.02 & 0.0200 & 0.0000 & & & & & \\
\hline & A034 & & & & & & 0.025 & 0.025 & 0.025 & 0.0250 & 0.0000 & & & & & \\
\hline & A 035 & 0.0217 & & & 0.0217 & & 0.0299 & 0.0284 & 0.0063 & 0.0215 & 0.0132 & & & & & \\
\hline & A036 & $<0206$ & $<0.206$ & $<0206$ & $<0.206$ & & $<0247$ & $<0.247$ & $<0247$ & $<0247$ & & & & & & \\
\hline & A 037 & $<0.05$ & $<0.05$ & $<0.05$ & $<0.05$ & & $<0.05$ & $<0.05$ & $<0.05$ & $<0.05$ & & & & & & \\
\hline & A038 & 0.0189 & 0.0185 & 0.018 & 0.0185 & 0.0005 & 0.0255 & 0.0249 & 0.025 & 0.0251 & 0.0003 & & & & & \\
\hline & A 039 & 0.02 & 0.01 & 0.01 & 0.0133 & 0.0058 & 0.02 & 0.02 & 0.02 & 0.0200 & 0.0000 & & & & & \\
\hline & A040 & & & & & & & & & & & 0.01653 & 0.01727 & 0.01718 & 0.0170 & 0.0004 \\
\hline & A 041 & 0 & 0 & 0 & 0.0000 & 0.0000 & 0 & 0 & 0 & 0.0000 & 0.0000 & & & & & \\
\hline & A043 & 0.036 & 0.037 & 0.035 & 0.0360 & 0.0010 & 0.056 & 0.056 & 0.057 & 0.0563 & 0.0006 & & & & & \\
\hline & A 044 & & & & & & & & & & & & & & & \\
\hline & A045 & & & & & & & & & & & & & & & \\
\hline & A 046 & 0 & 0 & 0 & 0.0000 & 0.0000 & 0 & 0 & 0 & 0.0000 & 0.0000 & & & & & \\
\hline & A050 & $<0.01$ & $<0.01$ & $<0.01$ & $<0.01$ & & 0.0154 & 0.0133 & 0.0146 & 0.0144 & 0.0011 & & & & & \\
\hline & A 051 & & & & & & & & & & & & & & & \\
\hline & A052 & & & & & & & & & & & & & & & \\
\hline & A 053 & & & & & & & & & & & & & & & \\
\hline & A054 & 0.01 & 0.01 & 0.01 & 0.0100 & 0.0000 & 0.01 & 0.01 & 0.01 & 0.0100 & 0.0000 & & & & & \\
\hline & $\mathrm{A} 055$ & 0.0137 & 0.014 & 0.0135 & 0.0137 & 0.0003 & 0.0224 & 0.0219 & 0.0224 & 0.0222 & 0.0003 & & & & & \\
\hline 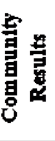 & & $\begin{array}{l}\text { Consensu } \\
\text { Consensu } \\
\text { Maximum } \\
\text { Minimum } \\
\text { N }\end{array}$ & $\begin{array}{l}\text { is Mean } \\
\text { is Standard I } \\
\text { a }\end{array}$ & Deviation & $\begin{array}{c}0.0165 \\
0.0011 \\
0.0800 \\
0.0000 \\
33\end{array}$ & & $\begin{array}{l}\text { Consensus } \\
\text { Consensus } \\
\text { Maximum } \\
\text { Minimum } \\
\text { N }\end{array}$ & $\begin{array}{l}\text { is Mean } \\
\text { is Standard } \\
\text { a }\end{array}$ & Deviation & $\begin{array}{c}0.0207 \\
0.0011 \\
0.1100 \\
0.0000 \\
41\end{array}$ & & $\begin{array}{l}\text { Consensus } \\
\text { Consensus } \\
\text { Maximum } \\
\text { Minimum } \\
\mathrm{N}\end{array}$ & $\begin{array}{l}\text { Mean } \\
\text { Standand }\end{array}$ & Deviation & $\begin{array}{c}0.0203 \\
0.0019 \\
0.0289 \\
0.0123 \\
9\end{array}$ & \\
\hline
\end{tabular}




\begin{tabular}{|c|c|c|c|c|c|c|c|c|c|c|c|c|c|c|c|c|c|}
\hline & & & & & & & & & Canr & nsbinol (C & (BN) & & & & & & \\
\hline & & & & He=p & p Oill (n) & ss \%) & & & He=p & p OAl 2 (na & ss \%) & & & Henp & OA 2a (n: & ss \%) & \\
\hline & & Lab & $\mathbf{A}$ & B & $\mathbf{C}$ & Avg & SD & $\mathbf{A}$ & B & $\mathbf{C}$ & Avg & SD & $\mathbf{A}$ & B & $\mathbf{C}$ & Avg & SD \\
\hline & & $\begin{array}{l}\text { NIST } \\
\text { A.056 }\end{array}$ & 0 & & & $\begin{array}{l}0.0160 \\
0.0000\end{array}$ & 0.0020 & 0.02386 & & & $\begin{array}{l}0.0240 \\
0.0239\end{array}$ & 0.0040 & & & & 0.0170 & 0.0040 \\
\hline & & A 057 & & & & & & & & & & & & & & & \\
\hline & & A058 & & 0.021 & 0.072 & 0.0465 & 0.0361 & & 0.029 & 0.046 & 0.0375 & 0.0120 & & & & & \\
\hline & & A 059 & 0.018 & 0.018 & 0.018 & 0.0180 & 0.0000 & 0.024 & 0.024 & 0.025 & 0.0243 & 0.0006 & & & & & \\
\hline$\square$ & & $A 060$ & $<0.02$ & $<0.02$ & $<0.02$ & $<0.02$ & & 0.02 & 0.02 & 0.02 & 0.0200 & 0.0000 & & & & & \\
\hline$\vec{\sim}$. & & A 061 & 0.016 & 0.016 & 0.016 & 0.0160 & 0.0000 & 0.021 & 0.02 & 0.02 & 0.0203 & 0.0006 & & & & & \\
\hline 0 & & A062 & & & & & & & & & & & 0.023 & 0.022 & 0.023 & 0.0227 & 0.0006 \\
\hline$\Xi$ & & A 063 & & & & & & 0.02026 & 0.02116 & 0.0205 & 0.0206 & 0.0005 & & & & & \\
\hline$\underline{\sigma}$ & & A064 & & & & & & & & & & & & & & & \\
\hline$\vec{\Omega}$ & & A 066 & yes & yes & yes & & & & & & & & & & & & \\
\hline$\stackrel{2}{\Rightarrow}$ & & A067 & & & & & & & & & & & & & & & \\
\hline 0 & & A 068 & & & & & & & & & & & & & & & \\
\hline 毛 & & $A 071$ & 0.08 & & & 0.0800 & & 0.11 & & & 0.1100 & & & & & & \\
\hline$\ddot{\infty}$. & & A 072 & & & & & & & & & & & & & & & \\
\hline טم & & A073 & 0.018 & 0.019 & 0.019 & 0.0187 & 0.0006 & 0.025 & 0.025 & 0.026 & 0.0253 & 0.0006 & & & & & \\
\hline$\underset{\Omega}{2}$ & & A 074 & 0.015 & 0.014 & 0.016 & 0.0150 & 0.0010 & 0.02 & 0.02 & 0.02 & 0.0200 & 0.0000 & & & & & \\
\hline ڤ. & & A075 & $<0.001$ & $<0.001$ & $<0.001$ & $<0.001$ & & $<0.001$ & $<0.001$ & $<0.001$ & $<0.001$ & & & & & & \\
\hline ט & & A 076 & 0.000439 & 0.000448 & 0.000446 & 0.0004 & 0.0000 & 0.000500 & 0.000511 & 0.000511 & 0.0005 & 0.0000 & & & & & \\
\hline$\tilde{\sigma}$ & & $\mathrm{A} 077$ & & & & & & ND & ND & ND & & & & & & & \\
\hline $\bar{D}$ & & A 079 & BLQ & BLQ & BLQ & & & BLQ & BLQ & BLQ & & & & & & & \\
\hline 曰 & & A081 & 0.027 & 0.027 & 0.026 & 0.0267 & 0.0006 & 0.033 & 0.034 & 0.033 & 0.0333 & 0.0006 & & & & & \\
\hline 8 & & A 082 & & & & & & & & & & & 0.02 & & & 0.0200 & \\
\hline CD & & A083 & & & & & & 0.02 & & & 0.0200 & & & & & & \\
\hline$\stackrel{0}{n}$ & & A 084 & 0.012 & 0.011 & 0.011 & 0.0113 & 0.0006 & 0.012 & 0.012 & 0.012 & 0.0120 & 0.0000 & & & & & \\
\hline 0 & & A085 & 0.02 & 0.02 & 0.02 & 0.0200 & 0.0000 & 0.02 & 0.02 & 0.02 & 0.0200 & 0.0000 & & & & & \\
\hline$\ddot{0}$ & 昰 & A 086 & & & & & & BLQ & BLQ & BLQ & & & & & & & \\
\hline$\stackrel{2}{=}$ & 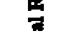 & A087 & & & & & & & & & & & & & & & \\
\hline co & 胥 & A 088 & & & & & & & & & & & & & & & \\
\hline$\rightarrow$ & 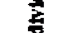 & A089 & 0.02 & 0.02 & 0.02 & 0.0200 & 0.0000 & 0.02 & 0.02 & $<0.01$ & 0.0200 & 0.0000 & & & & & \\
\hline 5 & 量 & A 090 & & & & & & & & & & & 0.0161 & 0.0162 & 0.0163 & 0.0162 & 0.0001 \\
\hline$=$ & & A091 & & & & & & & & & & & & & & & \\
\hline . & & A 092 & 0.0163 & 0.0164 & 0.0166 & 0.0164 & 0.0002 & 0.0198 & 0.0199 & 0.0201 & 0.0199 & 0.0002 & & & & & \\
\hline$\underline{\Xi}$ & & $\mathbf{A} 093$ & 0.02 & 0.02 & 0.02 & 00200 & 0.0000 & 0.03 & 0.03 & 0.02 & 0.0267 & 0.0058 & & & & & \\
\hline 릉 & & A 095 & $<0.0217$ & $<0.0217$ & $<0.0217$ & $<0.0217$ & & $<0.0217$ & $<0.0217$ & $<0.0217$ & $<0.0217$ & & & & & & \\
\hline 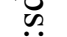 & & A096 & & & & & & & & & & & & & & & \\
\hline 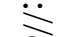 & & A 097 & 0.016 & 0.0154 & 0.0166 & 0.0160 & 0.0006 & 0.0202 & 0.0208 & 0.0207 & 0.0206 & 0.0003 & & & & & \\
\hline 를 & & A098 & $<0.0046$ & & & $<0.0046$ & & $<0.0025$ & & & $<0.0025$ & & & & & & \\
\hline$\stackrel{0}{.}$. & & A 099 & 0.011 & 0.011 & 0.011 & 0.0110 & 0.0000 & 0.016 & 0.015 & 0.015 & 0.0153 & 0.0006 & & & & & \\
\hline c & & A 100 & $<0.0210$ & $<0.0210$ & $<0.0210$ & $<0.0210$ & & $<0.0210$ & $<0.0210$ & $<0.0210$ & $<0.0210$ & & & & & & \\
\hline (có & & A 101 & & & & & & & & & & & & & & & \\
\hline$\Xi$ & & A 102 & $<0.02$ & $<0.02$ & $<0.02$ & $<0.02$ & & $<0.02$ & $<0.02$ & 0.02 & 0.0200 & & & & & & \\
\hline$\overline{0}$ & & A 103 & 0 & 0 & 0 & 0.0000 & 0.0000 & 0 & 0 & 0 & 0.0000 & 0.0000 & & & & & \\
\hline o & & A 104 & $<0.037$ & $<0.033$ & $<0.042$ & $<0.037$ & & $<0.033$ & $<0026$ & $<0.034$ & $<0.032$ & & & & & & \\
\hline 0 & & A 105 & $<0.025$ & $<0.025$ & $<0.025$ & $<0.025$ & & $<0.025$ & $<0.025$ & $<0.025$ & $<0.025$ & & & & & & \\
\hline$\infty$ & & A 106 & $<0.05$ & $<0.05$ & $<0.05$ & $<0.05$ & & $<0.05$ & $<0.05$ & $<0.05$ & $<0.05$ & & & & & & \\
\hline$\stackrel{\infty}{B}$ & & A 107 & 0.0137 & 0.0138 & 0.0147 & 0.0141 & 0.0006 & 0.0126 & 0.0124 & 0.012 & 0.0123 & 0.0003 & & & & & \\
\hline$Z$ & & A 108 & & & & & & & & & & & & & & & \\
\hline $\bar{U}$ & & A 109 & 0.02 & & & 0.0200 & & 0.02 & & & 0.0200 & & & & & & \\
\hline$\exists$ & & A110 & $<0.01$ & $<0.01$ & $<0.01$ & $<0.01$ & & $<0.01$ & $<0.01$ & $<0.01$ & $<0.01$ & & & & & & \\
\hline$\exists$ & & A 111 & 0.017 & 0.017 & 0.017 & 0.0170 & 0.0000 & 0.023 & 0.023 & 0.023 & 0.0230 & 0.0000 & & & & & \\
\hline 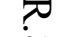 & & A112 & 0.0187 & 0.0182 & 0.0185 & 0.0185 & 0.0003 & 0.0265 & 0.0241 & 0.0253 & 0.0253 & 0.0012 & & & & & \\
\hline$\infty$ & & A 113 & 0 & 0 & 0 & 0.0000 & 0.0000 & 0 & 0 & 0 & 0.0000 & 0.0000 & & & & & \\
\hline$\infty$ & & A114 & & & & & & & & & & & & & & & \\
\hline u & & A 115 & & & & & & & & & & & 0.02 & 0.02 & 0.02 & 0.0200 & 0.0000 \\
\hline & & A116 & 0.06 & 0.0164 & 0.016 & 0.0308 & 0.0253 & 0.1 & 0.02066 & 0.0198 & 0.0468 & 0.0461 & & & & & \\
\hline & 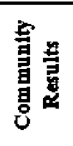 & & $\begin{array}{l}\text { Consensus } \\
\text { Consensus } \\
\text { Maximum } \\
\text { Minimum } \\
\mathrm{N}\end{array}$ & $\begin{array}{l}\text { is Mean } \\
\text { s Standard I }\end{array}$ & Deviation & $\begin{array}{c}0.0165 \\
0.0011 \\
0.0800 \\
0.0000 \\
33\end{array}$ & & $\begin{array}{l}\text { Consensus } \\
\text { Consensus } \\
\text { Maximum } \\
\text { Minimum } \\
\text { N }\end{array}$ & $\begin{array}{l}\text { s Mean } \\
\text { s Standard I }\end{array}$ & Deviation & $\begin{array}{c}0.0207 \\
0.0011 \\
0.1100 \\
0.0000 \\
41\end{array}$ & & $\begin{array}{l}\text { Consensu } \\
\text { Consensu } \\
\text { Maximum } \\
\text { Minimum } \\
\text { N }\end{array}$ & $\begin{array}{l}\text { Mean } \\
\text { Standand }\end{array}$ & Deviation & $\begin{array}{c}0.0203 \\
0.0019 \\
0.0289 \\
0.0123 \\
9\end{array}$ & \\
\hline
\end{tabular}




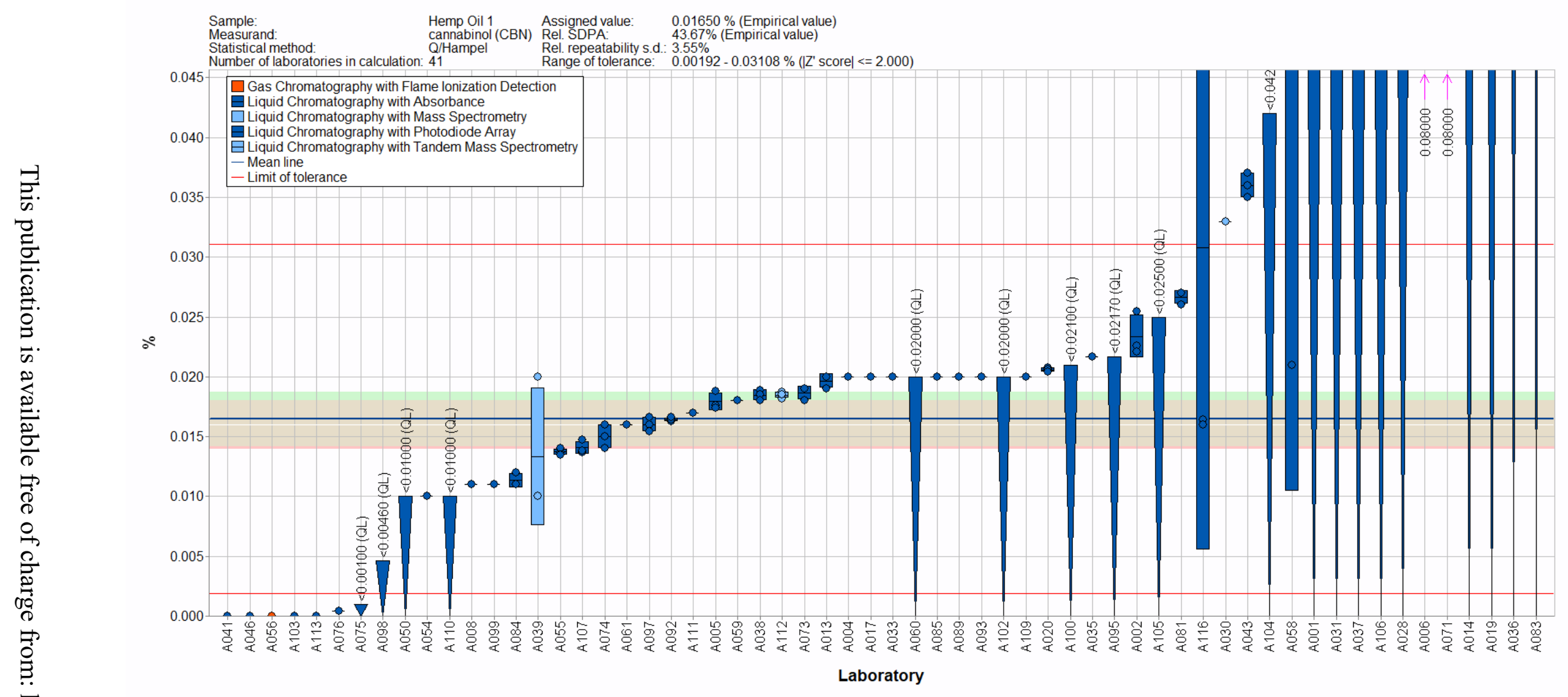

Figure 8-1. CBN in Hemp Oil 1 (data summary view - analytical method). In this view, individual laboratory data are plotted (circles) with the individual laboratory standard deviation (rectangle). The color of the data point represents the analytical method employed. The solid blue line represents the consensus mean, and the green shaded region represents the $95 \%$ confidence interval for the consensus mean. The solid red lines represent the consensus range of tolerance, calculated as the values above and below the consensus mean that result in an acceptable $Z_{\text {comm }}^{\prime}$ score, $\left|Z_{\text {comm }}^{\prime}\right| \leq 2$. The red shaded region represents the NIST range of tolerance, which encompasses the target value bounded by twice its uncertainty $\left(U_{\mathrm{NIST}}\right)$ and represents the range that results in an acceptable $Z_{\mathrm{NIST}}$ score, $\left|Z_{\mathrm{NIST}}\right| \leq 2$. The downward triangle represents data reported as a threshold or LOQ value. 


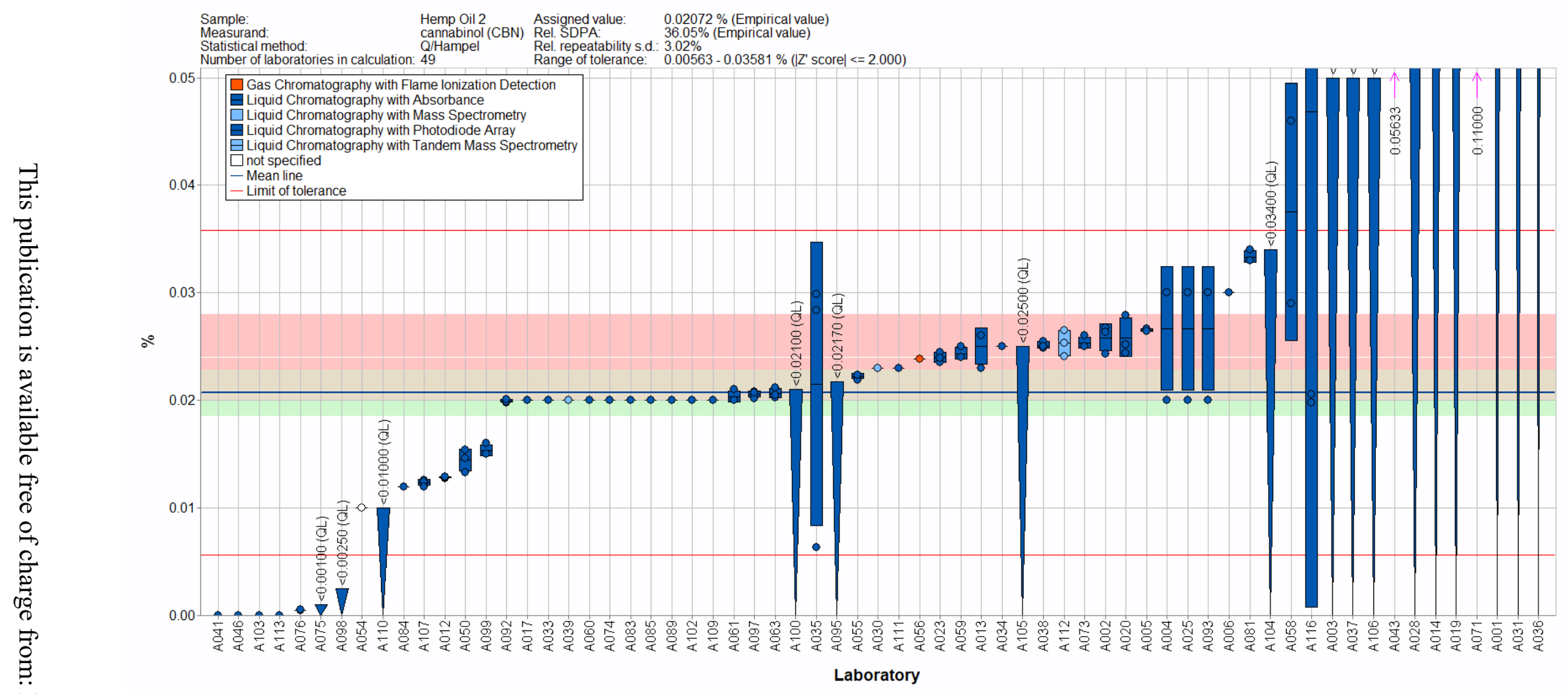

Figure 8-2. CBN in Hemp Oil 2 (data summary view - analytical method). In this view, individual laboratory data are plotted (circles) with the individual laboratory standard deviation (rectangle). The color of the data point represents the analytical method employed. The solid blue line represents the consensus mean, and the green shaded region represents the $95 \%$ confidence interval for the consensus mean. The solid red lines represent the consensus range of tolerance, calculated as the values above and below the consensus mean that result in an acceptable $Z_{\text {comm }}^{\prime}$ score, $\left|Z_{\text {comm }}^{\prime}\right| \leq 2$. The red shaded region represents the NIST range of tolerance, which encompasses the target value bounded by twice its uncertainty ( $U_{\text {NIST }}$ ) and represents the range that results in an acceptable $Z_{\text {NIST }}$ score, $\left|Z_{\text {NIST }}\right| \leq 2$. The shaded beige region represents the overlapping of the $95 \%$ confidence interval for the consensus mean (green region) and the NIST range of tolerance (red region). The downward triangle represents data reported as a threshold or LOQ value. 


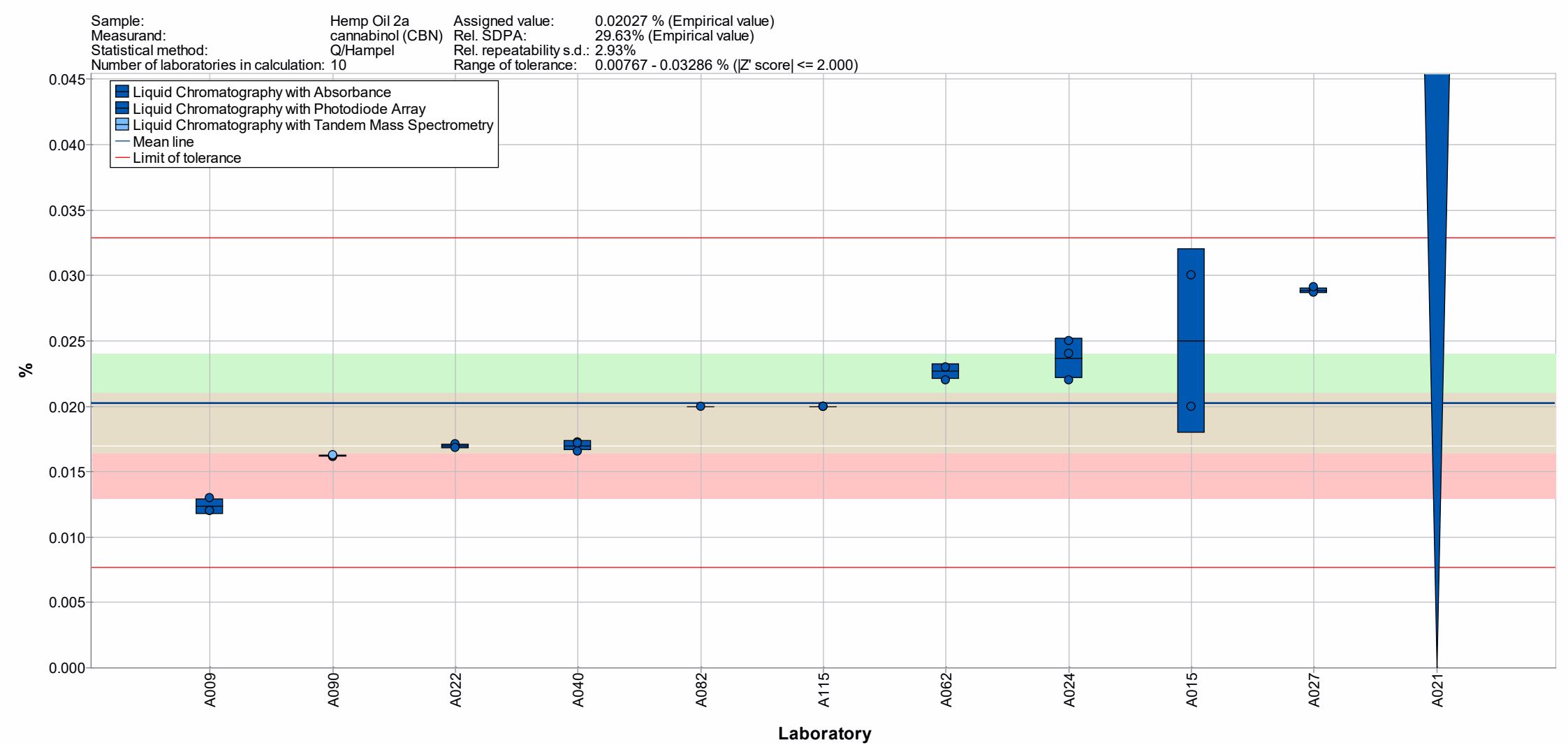

Figure 8-3. CBN in Hemp Oil 2a (data summary view - analytical method). In this view, individual laboratory data are plotted (circles) with the individual laboratory standard deviation (rectangle). The color of the data point represents the analytical method employed. The solid blue line represents the consensus mean, and the green shaded region represents the $95 \%$ confidence interval for the consensus mean. The solid red lines represent the consensus range of tolerance, calculated as the values above and below the consensus mean that result in an acceptable $Z_{\text {comm }}^{\prime}$ score, $\left|Z_{\text {comm }}^{\prime}\right| \leq 2$. The red shaded region represents the NIST range of tolerance, which encompasses the target value bounded by twice its uncertainty ( $U_{\text {NIST }}$ ) and represents the range that results in an acceptable $Z_{\text {NIST }}$ score, $\left|Z_{\text {NIST }}\right| \leq 2$. The shaded beige region represents the overlapping of the $95 \%$ confidence interval for the consensus mean (green region) and the NIST range of tolerance (red region). The downward triangle represents data reported as a threshold or LOQ value. 


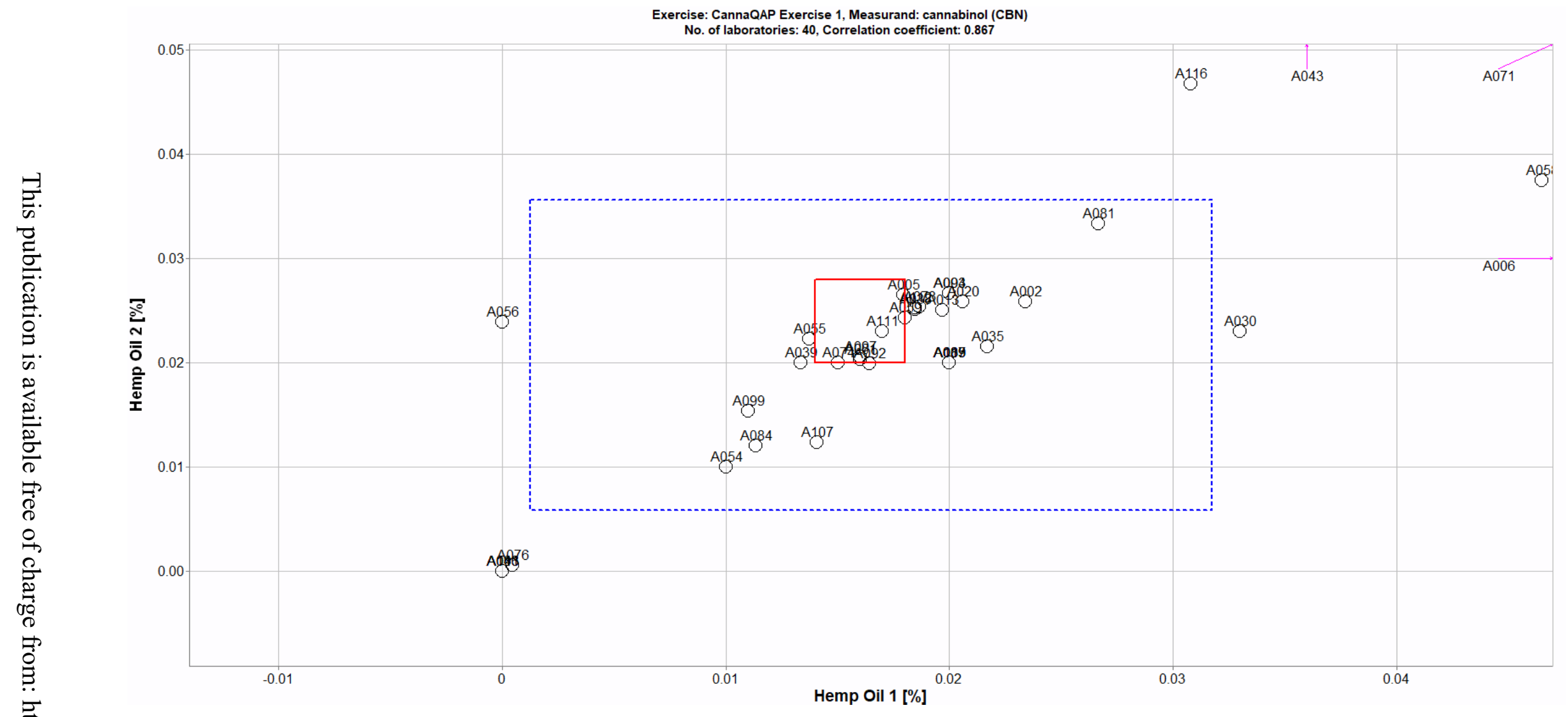

Figure 8-4. Laboratory means for CBN in Hemp Oil 1 and Hemp Oil 2 (sample/sample comparison view). In this view, the individual laboratory mean for one sample (Hemp Oil 1) is compared to the individual laboratory mean for a second sample (Hemp Oil 2). The solid red box represents the NIST range of tolerance for the two samples, Hemp Oil 1 (x-axis) and Hemp Oil 2 (y-axis), which encompasses the target values bounded by their uncertainties ( $U_{\text {NIST }}$ ) and represents the range that results in an acceptable $Z_{\text {NIST }}$ score, $\left|Z_{\mathrm{NIST}}\right| \leq 2$. The dotted blue box represents the consensus range of tolerance for Hemp Oil 1 (x-axis) and Hemp Oil 2 (y-axis), calculated as the values above and below the consensus means that result in an acceptable $Z_{\text {comm }}^{\prime}$ score, $\left|Z_{\text {comm }}^{\prime}\right| \leq 2$. 
Table 8-3. Data summary table for CBNA in hemp oils. Data highlighted in red have been flagged as a data entry of zero or results that include text (e.g., "< LOQ" or "present"). Data highlighted in blue have been identified as outside the consensus tolerance limits and would be estimated to result in an unacceptable $Z_{\text {comm }}^{\prime}$ score, $\left|Z_{\text {comm }}^{\prime}\right| \geq 2$.

\begin{tabular}{|c|c|c|c|c|c|c|c|c|c|c|c|c|c|c|c|c|}
\hline & \multirow[b]{3}{*}{ Lab } & \multicolumn{15}{|c|}{ Cannabinolic acid (CBNA) } \\
\hline & & \multicolumn{5}{|c|}{ Henp Oill (mass \%) } & \multicolumn{5}{|c|}{ Heap OAl 2 (mass \%) } & \multicolumn{5}{|c|}{ He=p O1 2a (mass \%) } \\
\hline & & $\mathbf{A}$ & $\mathbf{B}$ & $\mathbf{c}$ & Avg & SD & $\mathbf{A}$ & $\mathbf{B}$ & $\mathbf{c}$ & Avg & SD & $\mathbf{A}$ & $\mathbf{B}$ & $\mathbf{c}$ & Avg & SD \\
\hline \multirow{52}{*}{ 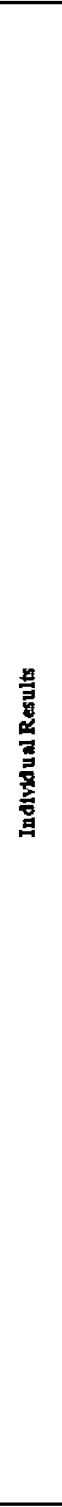 } & NIST & & & & & & & & & & & & & & & \\
\hline & $\mathrm{A} 002$ & $<0.0060$ & $<0.0060$ & $<0.0060$ & $<0.0060$ & & $<0.0060<$ & $<0.0060$ & $<0.0060$ & $<0.0060$ & & & & & & \\
\hline & A009 & & & & & & & & & & & $<0.00091$ & $<0.00091$ & $<0.00091$ & $<0.00091$ & \\
\hline & $\mathrm{A} 010$ & & & & & & & & & & & & & & & \\
\hline & A014 & $<0.09$ & & & $<0.09$ & & $<0.09$ & & & $<0.09$ & & & & & & \\
\hline & $\mathrm{A} 015$ & & & & & & & & & & & & & & & \\
\hline & A016 & & & & & & & & & & & & & & & \\
\hline & $\mathrm{A} 017$ & 0.01 & & & 0.0100 & & 0.03 & & & 0.030 & & & & & & \\
\hline & A019 & $<0.09$ & & & $<0.09$ & & $<0.09$ & & & $<0.09$ & & & & & & \\
\hline & $\mathrm{A} 020$ & 0.000544 & 0.000559 & 0.000383 & 0.0005 & 0.0001 & & & & & & & & & & \\
\hline & A021 & & & & & & & & & & & $<0.05$ & $<0.05$ & $<0.05$ & $<0.05$ & \\
\hline & $\mathrm{A} 022$ & & & & & & & & & & & & & & & \\
\hline & A024 & & & & & & & & & & & & & & & \\
\hline & $\mathrm{A} 025$ & & & & & & & & & & & & & & & \\
\hline & A027 & & & & & & & & & & & & & & & \\
\hline & A 031 & & & & & & & & & & & & & & & \\
\hline & A035 & $<0.0025$ & & & & & $<0.0025<$ & $<0.0025$ & $<0.0025$ & $<0.0025$ & & & & & & \\
\hline & A 040 & & & & & & & & & & & & & & & \\
\hline & A043 & 0.013 & 0.013 & 0.014 & 0.0133 & 0.0006 & $<0.002$ & $<0.002$ & $<0.002$ & $<0.002$ & & & & & & \\
\hline & A 044 & & & & & & & & & & & & & & & \\
\hline & A045 & & & & & & & & & & & & & & & \\
\hline & A 046 & 0 & 0 & 0 & 0.0000 & 0.0000 & 0 & 0 & 0 & 0.000 & 0.000 & & & & & \\
\hline & A052 & & & & & & & & & & & & & & & \\
\hline & $\mathrm{A} 053$ & & & & & & & & & & & & & & & \\
\hline & A055 & 0 & 0 & 0 & 0.0000 & 0.0000 & 0 & 0 & 0 & 0.000 & 0.000 & & & & & \\
\hline & A 057 & & & & & & & & & & & & & & & \\
\hline & A062 & & & & & & & & & & & & & & & \\
\hline & A 064 & & & & & & & & & & & & & & & \\
\hline & A066 & & & & & & & & & & & & & & & \\
\hline & A 068 & & & & & & & & & & & & & & & \\
\hline & A071 & & & & & & & & & & & & & & & \\
\hline & $\mathrm{A} 072$ & & & & & & & & & & & & & & & \\
\hline & A074 & $<0.008$ & $<0.008$ & $<0.008$ & $<0.008$ & & $<0.008$ & $<0.008$ & $<0.008$ & $<0.008$ & & & & & & \\
\hline & A 076 & & & & & & & & & & & & & & & \\
\hline & A082 & & & & & & & & & & & & & & & \\
\hline & A 083 & 0.03 & & & 0.0300 & & 0.06 & & & 0.060 & & & & & & \\
\hline & A084 & $<0.0125$ & $<0.0125$ & $<0.0125$ & $<0.0125$ & & $<0.0125<$ & $<0.0125$ & $<0.0125$ & $<0.0125$ & & & & & & \\
\hline & A 087 & & & & & & & & & & & & & & & \\
\hline & A 089 & 024 & 0.24 & 0.1 & 0.1933 & 0.0808 & 0.25 & 024 & 0.17 & 02200 & 0044 & & & & & \\
\hline & A 090 & & & & & & & & & & & & & & & \\
\hline & A092 & & & & & & & & & & & & & & & \\
\hline & A 093 & & & & & & & & & & & & & & & \\
\hline & A096 & & & & & & & & & & & & & & & \\
\hline & A 098 & $<0.0046$ & & & $<0.0046$ & & $<0.0025$ & & & $<0.0025$ & & & & & & \\
\hline & A099 & $<0.009$ & $<0.009$ & $<0.009$ & $<0.009$ & & $<0.009$ & $<0.009$ & $<0.009$ & $<0.009$ & & & & & & \\
\hline & A 101 & & & & & & & & & & & & & & & \\
\hline & A 102 & $<0.05$ & $<0.05$ & $<0.05$ & $<0.05$ & & $<0.05$ & $<0.05$ & $<0.05$ & $<0.05$ & & & & & & \\
\hline & A 107 & & & & & & & & & & & & & & & \\
\hline & A 108 & & & & & & & & & & & & & & & \\
\hline & A 112 & & & & & & & & & & & & & & & \\
\hline & A113 & & & & & & & & & & & & & & & \\
\hline & A115 & & & & & & & & & & & & & & & \\
\hline 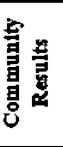 & & \begin{tabular}{|l} 
Consensur \\
Consensu \\
Maximum \\
Minimum \\
N
\end{tabular} & $\begin{array}{l}\text { is Mean } \\
\text { os Standard D } \\
\text { n }\end{array}$ & Deviation & $\begin{array}{c}0.0090 \\
0.0073 \\
0.1933 \\
0.0000 \\
5\end{array}$ & & \begin{tabular}{|l} 
Consensus $\mathrm{N}$ \\
Consensus S \\
Maximum \\
Minimum \\
N
\end{tabular} & $\begin{array}{l}\text { Mean } \\
\text { Standard }\end{array}$ & Deviation & $\begin{array}{c}0.033 \\
0.020 \\
0.220 \\
0.000 \\
3\end{array}$ & & \begin{tabular}{|l} 
Consensus \\
Consensus \\
Maximum \\
Minimum \\
N
\end{tabular} & $\begin{array}{l}\text { s Mean } \\
\text { s Standand I } \\
\end{array}$ & Deviation & & \\
\hline
\end{tabular}




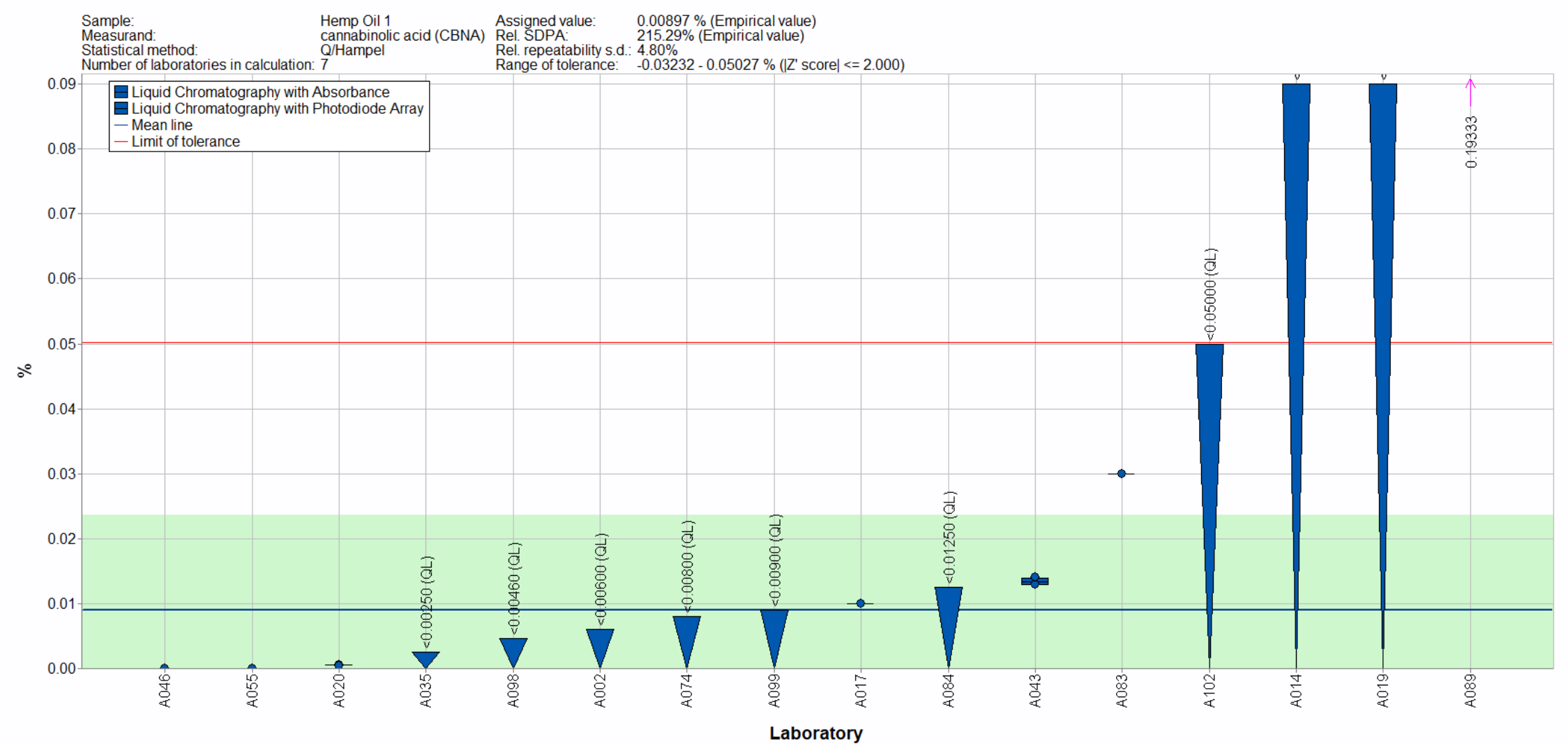

Figure 8-5. CBNA in Hemp Oil 1 (data summary view - analytical method). In this view, individual laboratory data are plotted (circles) with the individual laboratory standard deviation (rectangle). The color of the data point represents the analytical method employed. The solid blue line represents the consensus mean, and the green shaded region represents the $95 \%$ confidence interval for the consensus mean. The solid red line represents the upper consensus range of tolerance, calculated as the values above the consensus mean that results in an acceptable $Z_{\text {comm }}^{\prime}$ score, $\left|Z_{\text {comm }}^{\prime}\right| \leq 2$, with the lower limit set at zero. A NIST value has not been determined in this material. The downward triangle represents data reported as a threshold or LOQ value. 


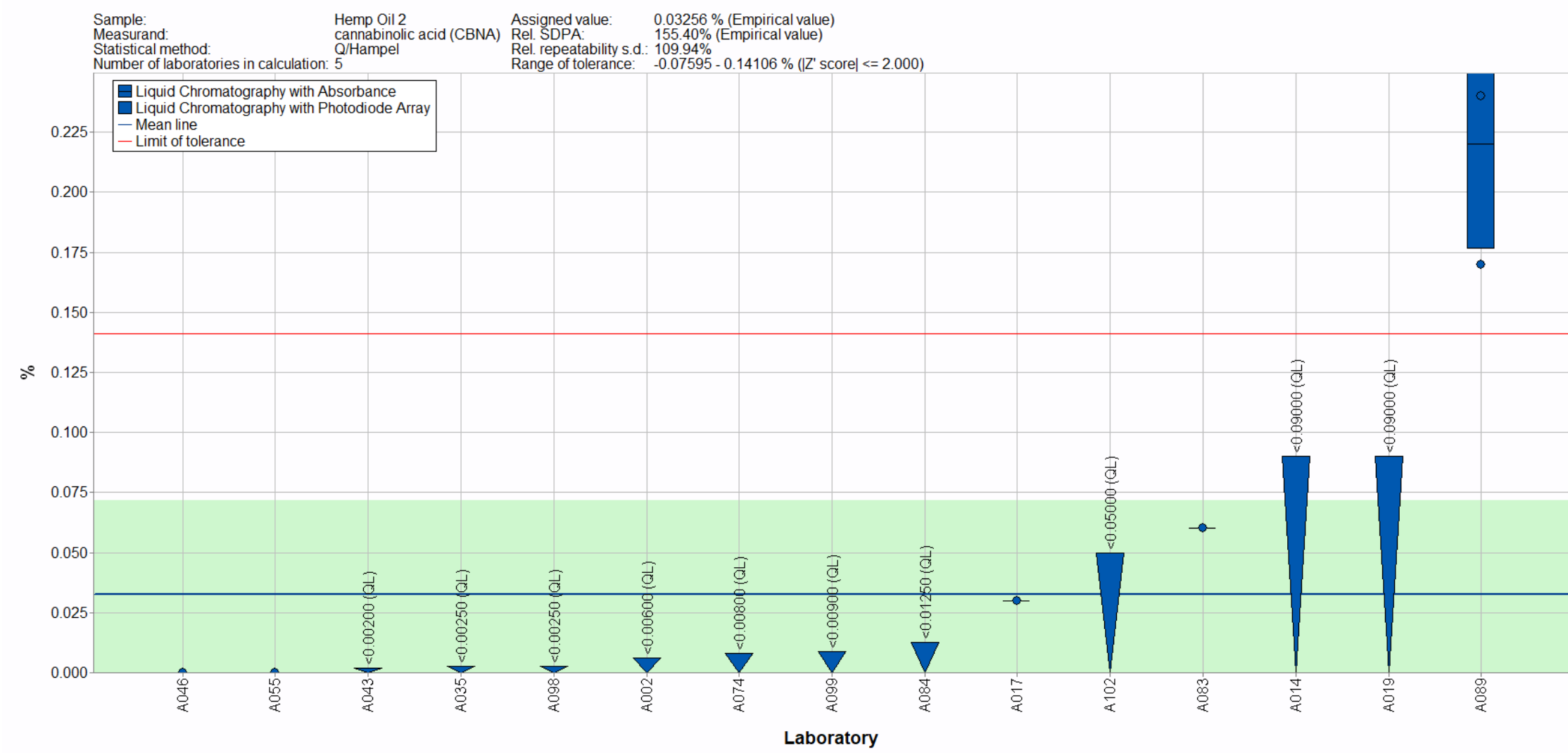

Figure 8-6. CBNA in Hemp Oil 2 (data summary view - analytical method). In this view, individual laboratory data are plotted (circles) with the individual laboratory standard deviation (rectangle). The color of the data point represents the analytical method employed. The solid blue line represents the consensus mean, and the green shaded region represents the $95 \%$ confidence interval for the consensus mean. The solid red line represents the upper consensus range of tolerance, calculated as the values above the consensus mean that results in an acceptable $Z_{\text {comm }}^{\prime}$ score, $\left|Z_{\text {comm }}^{\prime}\right| \leq 2$, with the lower limit set at zero. A NIST value has not been determined in this material. The downward triangle represents data reported as a threshold or LOQ value. 


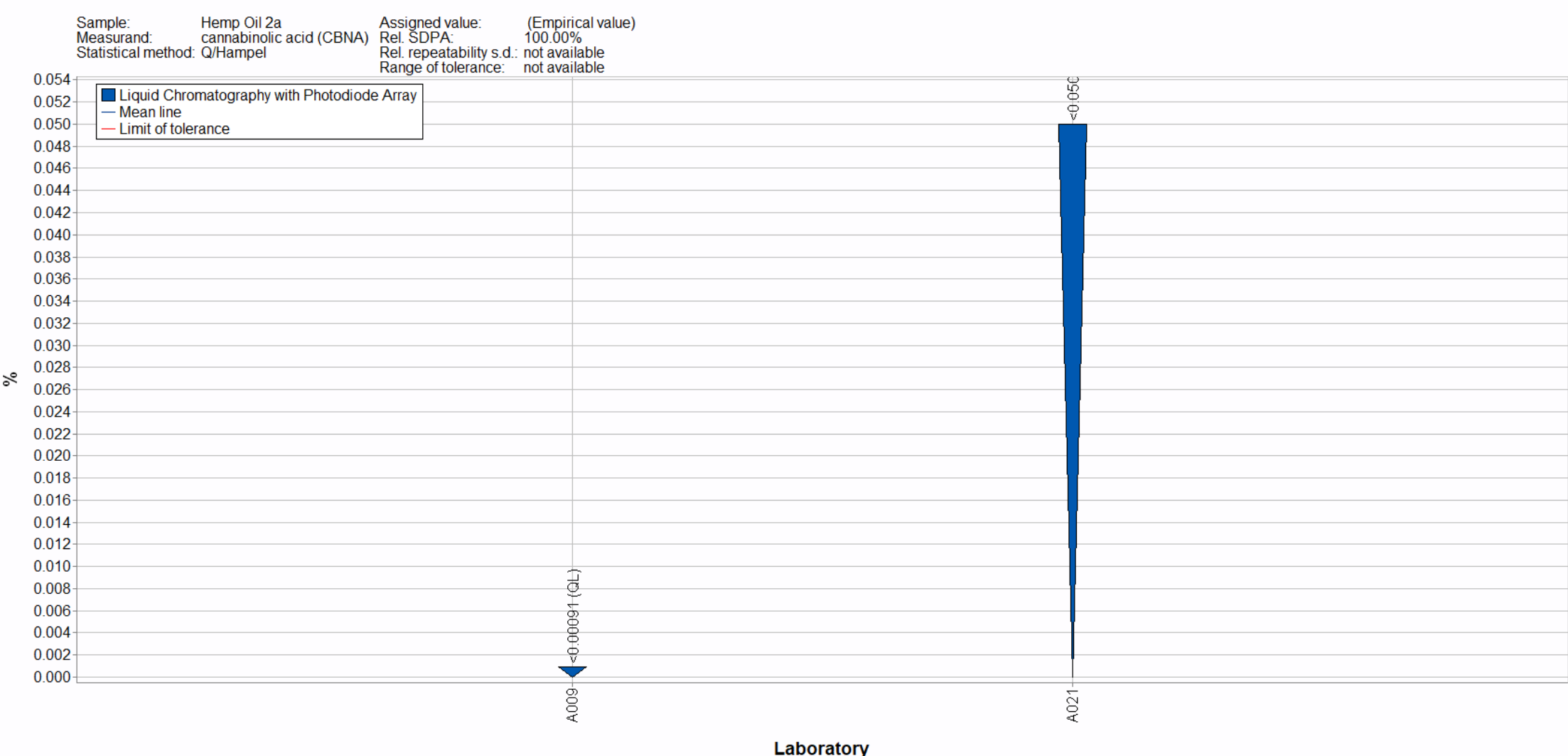

Figure 8-7. CBNA in Hemp Oil 2a (data summary view - analytical method). In this view, individual laboratory data are plotted (circles) with the individual laboratory standard deviation (rectangle). The color of the data point represents the analytical method employed. The downward triangle represents data reported as a threshold or LOQ value. 


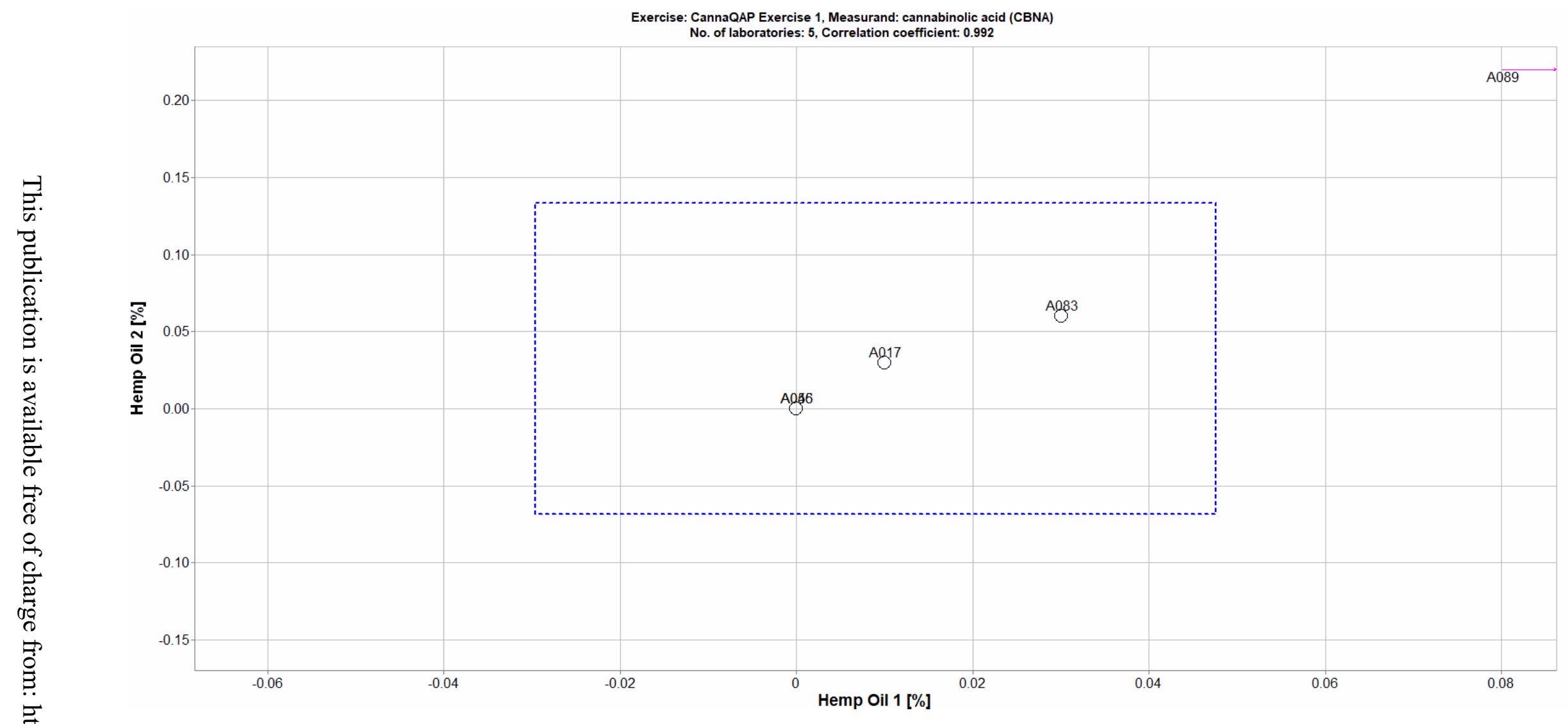

Figure 8-8. Laboratory means for CBNA in Hemp Oil 1 and Hemp Oil 2 (sample/sample comparison view). In this view, the individual laboratory mean for one sample (Hemp Oil 1) is compared to the individual laboratory mean for a second sample (Hemp Oil 2). The dotted blue box represents the consensus range of tolerance for Hemp Oil 1 (x-axis) and Hemp Oil 2 (y-axis), calculated as the values above and below the consensus means that result in an acceptable $Z_{\text {comm }}^{\prime}$ score, $\left|Z_{\text {comm }}^{\prime}\right| \leq 2$. 


\section{SECTION 9: THCV AND THCVA}

Study Overview

THCV is similar in structure to $\Delta^{9}$-THC with similar psychoactive properties. THCV is often detected at low levels in Cannabis plants and Cannabis-derived products and research has shown potential health for humans. ${ }^{\text {Error! Bookmark not defined. } 13} \mathrm{THCV}$ is synthesized in the plant from CBGA s imilar to $\Delta^{9}$-THC instead of its acidic precursor THCVA, which is produced from the breakdown of CBGVA through decarboxylation with exposure of heat or light. Participants in this study were asked to use in-house analytical methods to determine the mass fraction (\%) of THCV and THCVA in the three hemp oils. The preparation of these hemp oils included a decarboxylation step permitting the potential presence of THCV and THCVA in these samples.

\section{$\underline{\text { Reporting Statistics }}$}

- The enrollment and reporting statistics for THCV and THCVA are described in the table below for each analyte. Reported values may include non-quantitative results (zero or below LOQ).

\begin{tabular}{cccccccc} 
& \multicolumn{2}{c}{ Hemp Oil 1 } & \multicolumn{2}{c}{ Hemp Oil 2 } & & \multicolumn{2}{c}{ Hemp Oil 2a } \\
& $\underline{\text { Number of }}$ & $\underline{\underline{\text { Reporting }}}$ & & $\underline{\text { Number of }}$ & $\underline{\underline{\text { Reporting }}}$ & $\underline{\text { Number of }}$ & $\underline{\underline{\text { Reporting }}}$ \\
$\underline{\text { Analyte }}$ & $\underline{\text { Participants }}$ & $\underline{\text { Results }}$ & $\underline{\text { Participants }}$ & $\underline{\underline{\text { Results }}}$ & $\underline{\text { Participants }}$ & $\underline{\text { Results }}$ \\
THCV & 54 & $67 \%$ & 62 & $66 \%$ & 19 & $47 \%$ \\
THCVA & 36 & $58 \%$ & 38 & $58 \%$ & 19 & $16 \%$
\end{tabular}

- Most laboratories reported using solvent extraction or sample dilution for determination of THCV and THCVA in the three hemp oil samples (see table below). Additional sample preparation details are summarized at the end of the report in Appendix I.

\begin{tabular}{|c|c|c|}
\hline$\underline{\text { Reported }}$ & \multicolumn{2}{|c|}{ Percent Reporting } \\
\hline Preparation Method & $\underline{\mathrm{THCV}}$ & $\underline{\text { THCVA }}$ \\
\hline Solvent Extraction & 69.9 & 72.9 \\
\hline Dilution & 26.2 & 25.4 \\
\hline Other & 0.0 & 0.0 \\
\hline None & 1.9 & 1.7 \\
\hline No Response & 1.9 & 0.0 \\
\hline
\end{tabular}

\footnotetext{
${ }^{13}$ J McPartland, M Duncan, V Marzo, R Pertwee. Br J Pharmacol. 172: 737-753 (2015) https://doi.org/10.111/bph.12944.
} 
- Most laboratories reported using LC-PDA or LC-UV for the determination of THCV and THCVA in the three hemp oil samples (see table below). Additional method details are summarized at the end of the report in Appendix I.

\begin{tabular}{|c|c|c|}
\hline Reported & \multicolumn{2}{|c|}{ Percent Reporting } \\
\hline Analytical Method & $\underline{\mathrm{THCV}}$ & $\underline{\text { THCVA }}$ \\
\hline LC-PDA & 65.0 & 61.0 \\
\hline LC-UV & 27.2 & 33.9 \\
\hline LC-MS & 1.0 & 1.7 \\
\hline LC-MS/MS & 4.9 & 0.0 \\
\hline GC-FID & 0.0 & 0.0 \\
\hline GC-MS & 0.0 & 0.0 \\
\hline Other & 1.9 & 3.4 \\
\hline
\end{tabular}

$\underline{\text { Study Results }}$

THCV

- No target means or ranges were provided in Table 9-1 for THCV in the three hemp oils.

- The consensus means and ranges for THCV are summarized in Figure 9-1, Figure 9-2, and Figure 9-3 for Hemp Oil 1, Hemp Oil 2, and Hemp Oil 2a, respectively. Data from participants submitting only one measurement were included in Table 9-2 but were not included in the calculation of consensus statistics. ${ }^{2}$

- A comparison of individual laboratory means for THCV in Hemp Oil 1 and Hemp Oil 2 is summarized in Figure 9-4 for laboratories who reported results for both samples.

THCVA

- No target means or ranges were provided in Table 9-1 for THCVA in the three hemp oils.

- The consensus means and ranges for THCVA are summarized in Figure 9-5, Figure 9-6, and Figure 9-7 for Hemp Oil 1, Hemp Oil 2, and Hemp Oil 2a, respectively. Data from participants submitting only one measurement were included in Table 9-3 but were not included in the calculation of consensus statistics. ${ }^{2}$

- A comparison of individual laboratory means for THCVA in Hemp Oil 1 and Hemp Oil 2 is summarized in Figure 9-8 for laboratories who reported results for both samples.

Overall

- The between-laboratory variabilities for determination of THCV and THCVA in the hemp oil samples are shown in the table below.

Between-Laboratory Variability (\% RSD)

\begin{tabular}{|c|c|c|c|}
\hline Analyte & Hemp Oil 1 & Hemp Oil 2 & Hemp Oil 2a \\
\hline THCV & 59.4 & 29.2 & 303.1 \\
\hline THCVA & 133.0 & 83.8 & NA \\
\hline
\end{tabular}


Study Discussion and Technical Recommendations

The following recommendations are based on results obtained from the participants in this study.

THCV

- Approximately $20 \%$ of the laboratories reporting results for THCV provided values outside the consensus ranges for both Hemp Oil 1 and Hemp Oil 2 (Figure 9-4).

- Laboratories reporting results above the consensus mean in Hemp Oil 1 also reported results above the consensus mean for Hemp Oil 2. Trends of this type often indicate a calibration bias.

- Most laboratories reported that THCV was present in the samples at or below their LOQ (nonzero values). The low levels resulted in large consensus ranges and between-laboratory variabilities (29\% to $303 \%)$.

- Approximately $6 \%$ of the laboratories reporting results used LC-MS or LC-MS/MS methods with some having low enough LOQs to determine THCV at the consensus level in Hemp Oil 1, Hemp Oil 2, and Hemp Oil 2a.

- Approximately $92 \%$ of the laboratories reporting results used LC-UV or LC-PDA methods with only $31 \%$ and $26 \%$ of these laboratories with low enough LOQs to determine THCV at the consensus levels in Hemp Oil 1 and Hemp Oil 2, respectively.

- No additional trends were observed for the sample preparation, analytical methods, and/or analytical parameters reported for THCV in the three hemp oil samples.

\section{THCVA}

- Most laboratories reported that THCVA was present in the samples at or below their LOQ (non-zero values). The low levels resulted in large consensus ranges and between-laboratory variabilities ( $84 \%$ to $133 \%)$.

- Approximately $2 \%$ of the laboratories reporting results used LC-MS or LC-MS/MS methods with none having low enough LOQs to determine THCVA at the consensus levels in Hemp Oil 1, Hemp Oil 2, and Hemp Oil 2a.

- Approximately $95 \%$ of the laboratories reporting results used LC-UV or LC-PDA methods with only $5 \%$ and $9 \%$ of these laboratories with low enough LOQs to determine THCVA at the consensus levels in Hemp Oil 1 and Hemp Oil 2, respectively.

- No additional trends were observed for the sample preparation, analytical methods, and/or analytical parameters reported for THCVA in the three hemp oil samples.

Overall

- Proper storage conditions are important for hemp oils because THCVA can readily convert to THCV when stored at elevated or room temperatures.

- Participants were asked to store the samples under controlled refrigeration $\left(\approx 4^{\circ} \mathrm{C}\right)$.

- Laboratories should perform in-house evaluations of their storage conditions through stability test of their hemp oils at various environmental factors such as temperature, humidity, and light exposure. ${ }^{5}$

- Over 100 cannabinoids have been identified in Cannabis plant samples with similarities in structure and molecular mass. As a result, chromatographic peak identity should always be confirmed using appropriate reference spectra of pure standards for cannabinoids and is particularly important for cannabinoids that are present at levels close to the method LOQ. Analytical methods should be able to clearly distinguish between cannabinoids. 
- The use of appropriate calibration materials and quality assurance samples to establish that a method is in control and being performed correctly may reduce the likelihood of outlying data. Quality assurance samples can be commercially available reference materials (CRMs, SRMs, or RMs) or materials prepared in-house.

- Measurement results should be reported accurately.

- Reported values should be the mass fraction (\%) of the THCV and THCVA in the three hemp oil samples. Participants who reported values based on a volume fraction (\%) for Hemp Oil 2 or Hemp Oil 2a should use the density of the sample for the conversion to mass fraction. Hemp Oil 1 was too viscous to prepare dilutions by volume.

- Zero is not a quantity that can be measured. If values are below LOQ, results should be reported as such. A more appropriate result would be to report that a value is below the LOQ (e.g., "“<0.02”).

- Laboratories reporting results based on a certain threshold should enter the numerical threshold (e.g., "< 1").

- Laboratories reporting results flagged as outliers should check for calculation errors when preliminary data tables are sent for inspection. One example is to confirm that factors for all dilutions have been properly tabulated or that results are reported in the requested units. 
Table 9-1. Individualized data summary table (NIST) for THCV and THCVA in hemp oils.

\section{National Institute of Standards and Technology}

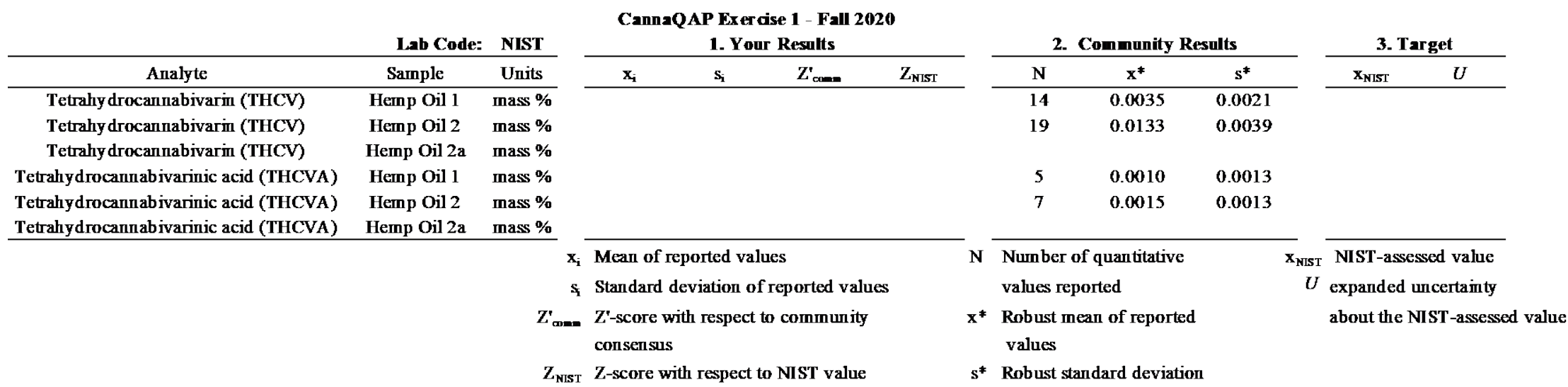


Table 9-2. Data summary table for THCV in hemp oils. Data highlighted in red have been flagged as a data entry of zero or results that include text (e.g., "< LOQ" or "present"). Data highlighted in blue have been identified as outside the consensus tolerance limits and would be estimated to result in an unacceptable $Z_{\text {comm }}^{\prime}$ score, $\left|Z_{\text {comm }}^{\prime}\right| \geq 2$. Note: This table spans two pages; the NIST values and consensus values are included on both pages for convenience.

\begin{tabular}{|c|c|c|c|c|c|c|c|c|c|c|c|c|c|c|c|c|}
\hline & \multirow[b]{3}{*}{ Lab } & \multicolumn{15}{|c|}{ Tetrahydrocannabivarin (THCV) } \\
\hline & & \multicolumn{10}{|c|}{ Heap Oill (mass \%) } & \multicolumn{5}{|c|}{ Heap O1 2 (mass \%) } \\
\hline & & $\mathbf{A}$ & $\mathbf{B}$ & $\mathbf{c}$ & Avg & SD & $\mathbf{A}$ & $\mathbf{B}$ & $\mathbf{c}$ & Avg & SD & $\mathbf{A}$ & $\mathbf{B}$ & $\mathbf{c}$ & Avg & SD \\
\hline \multirow{39}{*}{ 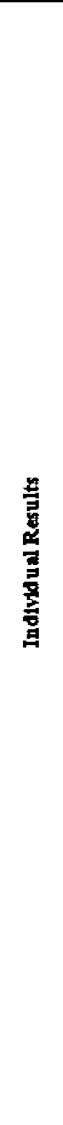 } & NIST & & & & & & & & & & & & & & & \\
\hline & $\mathrm{A} 002$ & $<0.0069$ & $<0.0069$ & $<0.0069$ & $<0.0069$ & & $<0.0069$ & $<0.0069$ & $<0.0069$ & $<0.0069$ & & & & & & \\
\hline & A003 & & & & & & $<0.01$ & $<0.01$ & $<0.01$ & $<0.01$ & & & & & & \\
\hline & $\mathrm{A} 005$ & 0 & 0 & 0 & 0.0000 & 0.0000 & 0 & 0 & 0 & 0.0000 & 0.0000 & & & & & \\
\hline & A006 & $<0.001$ & & & $<0.001$ & & $<0.001$ & & & $<0.001$ & & & & & & \\
\hline & A 007 & & & & & & & & & & & & & & & \\
\hline & A008 & & & & & & & & & & & & & & & \\
\hline & $\mathrm{A} 009$ & & & & & & & & & & & $<0.00205$ & $<0.00205$ & $<0.00205$ & $<0.00205$ & \\
\hline & A010 & & & & & & & & & & & & & & & \\
\hline & $\mathrm{A} 012$ & & & & & & 0 & 0 & 0 & 0.0000 & 0.0000 & & & & & \\
\hline & A013 & & & & & & & & & & & & & & & \\
\hline & $\mathrm{A} 014$ & $<0.09$ & & & $<0.09$ & & $<0.09$ & & & $<0.09$ & & & & & & \\
\hline & A015 & & & & & & & & & & & & & & & \\
\hline & A 016 & & & & & & & & & & & & & & & \\
\hline & A017 & 0.01 & & & 0.0100 & & 0.04 & & & 0.0400 & & & & & & \\
\hline & $\mathrm{A} 018$ & & & & & & & & & & & & & & & \\
\hline & A019 & $<0.09$ & & & $<009$ & & $<0.09$ & & & $<0.09$ & & & & & & \\
\hline & $\mathrm{A} 020$ & & & & & & & & & & & & & & & \\
\hline & A021 & & & & & & & & & & & $<0.05$ & $<0.05$ & $<0.05$ & $<0.05$ & \\
\hline & $\mathrm{A} 022$ & & & & & & & & & & & & & & & \\
\hline & A023 & & & & & & 0 & 0 & 0 & 0.0000 & 0.0000 & & & & & \\
\hline & A 024 & & & & & & & & & & & $<0.01$ & $<0.01$ & $<0.01$ & $<0.01$ & \\
\hline & A025 & & & & & & & & & & & & & & & \\
\hline & $\mathrm{A} 027$ & & & & & & & & & & & $<0.0057$ & $<0.0057$ & $<0.0057$ & $<0.0057$ & \\
\hline & A031 & & & & & & & & & & & & & & & \\
\hline & A 035 & 0.00633 & & & 0.0063 & & 0.0135 & 0.0115 & 0.0134 & 0.0128 & 0.0011 & & & & & \\
\hline & A036 & $<0.206$ & $<0.206$ & $<0.206$ & $<0206$ & & $<0247$ & $<0.247$ & $<0247$ & $<0247$ & & & & & & \\
\hline & A 038 & $<0.025$ & $<0.025$ & $<0.025$ & $<0.025$ & & $<0.025$ & $<0.025$ & $<0.025$ & $<0.025$ & & & & & & \\
\hline & A039 & 0 & 0 & 0 & 0.0000 & 0.0000 & 0 & 0 & 0 & 0.0000 & 0.0000 & & & & & \\
\hline & A 040 & & & & & & & & & & & ND & ND & ND & & \\
\hline & A041 & 0 & 0 & 0 & 0.0000 & 0.0000 & 0 & 0 & 0 & 0.0000 & 0.0000 & & & & & \\
\hline & A 043 & 0.009 & 0.006 & 0.009 & 0.0080 & 0.0017 & 0.011 & 0.011 & 0.011 & 0.0110 & 0.0000 & & & & & \\
\hline & A044 & & & & & & & & & & & & & & & \\
\hline & A 045 & & & & & & & & & & & & & & & \\
\hline & A046 & 0 & 0 & 0 & 0.0000 & 0.0000 & 0 & 0 & 0 & 0.0000 & 0.0000 & & & & & \\
\hline & $\mathrm{A} 050$ & 0.11 & 0.112 & 0.112 & 0.1113 & 0.0012 & $<0.01$ & $<0.01$ & $<0.01$ & $<0.01$ & & & & & & \\
\hline & A052 & & & & & & & & & & & & & & & \\
\hline & A 053 & & & & & & & & & & & & & & & \\
\hline & A055 & 0.0068 & 0.0056 & 0.0047 & 0.0057 & 0.0011 & 0 & 0 & 0 & 0.0000 & 0.0000 & & & & & \\
\hline 焉臂 & & \begin{tabular}{|l|} 
Consensus \\
Consensus \\
Maximum \\
Minimum \\
N
\end{tabular} & $\begin{array}{l}\text { s Mean } \\
\text { s Standard } \\
\end{array}$ & Deviation & $\begin{array}{c}0.0035 \\
0.0021 \\
4.0133 \\
0.0000 \\
14\end{array}$ & & $\begin{array}{l}\text { Consens } \\
\text { Consens } \\
\text { Maximun } \\
\text { Minimun } \\
\text { N }\end{array}$ & $\begin{array}{l}\text { is Mean } \\
\text { is Standard I } \\
\text { a }\end{array}$ & Deviation & $\begin{array}{c}0.0133 \\
0.0039 \\
8.4067 \\
0.0000 \\
19\end{array}$ & & \begin{tabular}{|l|} 
Consensus \\
Consensu \\
Maximum \\
Minimum \\
N
\end{tabular} & $\begin{array}{l}\text { S Mean } \\
s \text { Standand } \mathrm{D} \\
\end{array}$ & Deviation & $\begin{array}{c}0.0016 \\
0.0049 \\
0.0033 \\
0.0000 \\
1\end{array}$ & \\
\hline
\end{tabular}




\begin{tabular}{|c|c|c|c|c|c|c|c|c|c|c|c|c|c|c|c|c|c|}
\hline & & & & & & & & & etrahydro & Gnnabixa & in (THC) & & & & & & \\
\hline & & & & He= & Orl 1 (n) & ss \%) & & & Henp & Ol $2(\mathrm{~m}$ & ss \%) & & & He= & On 22 (n) & ss \%) & \\
\hline & & Lab & $\mathbf{A}$ & B & $\mathbf{C}$ & Avg & SD & $\mathbf{A}$ & $\mathbf{B}$ & $\mathbf{C}$ & Avg & SD & $\mathbf{A}$ & $\mathbf{B}$ & $\mathbf{C}$ & Avg & SD \\
\hline & & NIST & & & & & & & & & & & & & & & \\
\hline & & A 057 & & & & & & & & & & & & & & & \\
\hline & & A059 & 0 & 0 & 0 & 0.0000 & 0.0000 & 0 & 0 & 0 & 0.0000 & 0.0000 & & & & & \\
\hline & & A 060 & 3.99 & 4.02 & 4.03 & 4.0133 & 0.0208 & 8.39 & 8.36 & 8.47 & 8.4067 & 0.0569 & & & & & \\
\hline & & A.061 & 0.025 & 0.025 & 0.024 & 00247 & 0.0006 & 0.047 & 0.045 & $0 \_048$ & 0.0467 & 0.0015 & & & & & \\
\hline$\square$ & & A 062 & & & & & & & & & & & $<0.01$ & $<0.01$ & $<0.01$ & $<0.01$ & \\
\hline $\bar{\sigma}$. & & A063 & & & & & & 0.04136 & 0.03967 & 0.04099 & 0.0407 & 0.0009 & & & & & \\
\hline$\sigma$ & & A 064 & & & & & & & & & & & & & & & \\
\hline$\Xi$ & & A066 & & & & & & & & & & & & & & & \\
\hline$\sigma$ & & A 068 & & & & & & & & & & & & & & & \\
\hline$\overline{0}$ & & A071 & & & & & & & & & & & & & & & \\
\hline$\stackrel{2}{2}$ & & A 072 & & & & & & 0.12 & & & 0.1200 & & & & & & \\
\hline 0 & & $\mathrm{~A} 073$ & 0 & 0 & 0 & 0.0000 & 0.0000 & 0.012 & 0.011 & 0.012 & 0.0117 & 0.0006 & & & & & \\
\hline 包 & & A 074 & $<0.004$ & $<0.004$ & $<0.004$ & $<0.004$ & & $<0.004$ & $<0.004$ & $<0.004$ & $<0.004$ & & & & & & \\
\hline$\vec{\infty}$. & & A076 & & & 0000019 & 00000 & & & & & & & & & & & \\
\hline טמ & & A 077 & & & & & & ND & ND & ND & & & & & & & \\
\hline$\sum_{<}^{2}$ & & A082 & & & & & & & & & & & 0 & & & 0.0000 & \\
\hline ڤ. & & A 083 & $<0.25$ & & & $<0.25$ & & $<0.25$ & & & $<0.25$ & & & & & & \\
\hline 0 & & A084 & $<0.017$ & $<0.017$ & $<0.017$ & $<0.017$ & & $<0.017$ & $<0.017$ & $<0.017$ & $<0.017$ & & & & & & \\
\hline$\tilde{\sigma}$ & 当 & A 085 & $<0.025$ & $<0.025$ & $<0.025$ & $<0.025$ & & $<0.025$ & $<0.025$ & $<0.025$ & $<0.025$ & & & & & & \\
\hline $\bar{D}$ & 局 & A087 & & & & & & & & & & & & & & & \\
\hline 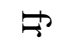 & 을 & A 088 & & & & & & & & & & & & & & & \\
\hline 8 & 寻 & A089 & $<0.01$ & $<0.01$ & $<0.01$ & $<0.01$ & & $<0.01$ & $<0.01$ & $<0.01$ & $<0.01$ & & & & & & \\
\hline C & 疍 & A 090 & & & & & & & & & & & 0.0031 & 0.0034 & 0.0033 & 0.0033 & 0.0002 \\
\hline$\stackrel{0}{h}$ & 吕 & A091 & & & & & & & & & & & & & & & \\
\hline 0 & & A 092 & & & & & & & & & & & & & & & \\
\hline $\overrightarrow{0}$ & & A093 & & & & & & & & & & & & & & & \\
\hline$\stackrel{0}{=}$ & & A 095 & $<0.0851$ & $<0.0851$ & $<0.0851$ & $<0.0851$ & & $<0.0851$ & $<0.0851$ & $<0.0851$ & $<0.0851$ & & & & & & \\
\hline 0 & & A096 & & & & & & & & & & & & & & & \\
\hline$\rightarrow$ & & A 098 & $<0.0046$ & & & $<0.0046$ & & $<0.0025$ & & & $<0.0025$ & & & & & & \\
\hline $\overrightarrow{0}$ & & A099 & $<0.017$ & $<0.017$ & $<0.017$ & $<0.017$ & & 0.025 & 0.029 & 0.025 & 0.0263 & 0.0023 & & & & & \\
\hline 当 & & A 100 & $<0.0210$ & $<0.0210$ & $<0.0210$ & $<0.0210$ & & $<0.0210$ & $<0.0210$ & $<0.0210$ & $<0.0210$ & & & & & & \\
\hline 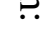 & & A 101 & & & & & & & & & & & & & & & \\
\hline$\Xi$ & & A 102 & $<0.02$ & $<0.02$ & $<0.02$ & $<0.02$ & & $<0.02$ & $<0.02$ & $<0.02$ & $<0.02$ & & & & & & \\
\hline 7 & & A 104 & $<0.037$ & $<0.033$ & $<0.042$ & $<0.037$ & & $<0.033$ & $<0.026$ & $<0.034$ & $<0.032$ & & & & & & \\
\hline 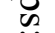 & & A 105 & $<0.025$ & $<0.025$ & $<0.025$ & $<0.025$ & & 0.04 & 0.05 & 0.06 & 0.0500 & 0.0100 & & & & & \\
\hline$\stackrel{\ominus}{\doteqdot}$ & & A 107 & 0.125 & 0.0129 & $<0.01$ & 0.0690 & 0.0793 & 0.0257 & 0.0255 & 0.0249 & 0.0254 & 0.0004 & & & & & \\
\hline 를 & & A 108 & & & & & & & & & & & & & & & \\
\hline$\stackrel{0}{.}$ & & A109 & & & & & & & & & & & & & & & \\
\hline 0 & & A 110 & $<0.01$ & $<0.01$ & $<0.01$ & $<0.01$ & & $<0.01$ & $<0.01$ & $<0.01$ & $<0.01$ & & & & & & \\
\hline$\theta$ & & A112 & 00021 & 0.002 & 0.0021 & 0.0021 & 0.0001 & 0.0052 & 0.0059 & 0.0056 & 0.0056 & 0.0004 & & & & & \\
\hline 2 & & A 113 & 0 & 0 & 0 & 0.0000 & 0.0000 & 0 & 0 & 0 & 0.0000 & 0.0000 & & & & & \\
\hline $\overrightarrow{0}$ & & A114 & & & & & & & & & & & & & & & \\
\hline o & & A 115 & & & & & & & & & & & $<0.01$ & $<0.01$ & $<0.01$ & $<0.01$ & \\
\hline$\underbrace{\infty}_{\infty}$ & 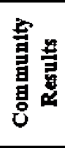 & & \begin{tabular}{|l|} 
Consensus \\
Consensus \\
Maximum \\
Minimum \\
$\mathrm{N}$ \\
\end{tabular} & $\begin{array}{l}\text { s Mean } \\
\text { s Stmdand }\end{array}$ & Deviation & $\begin{array}{c}0.0035 \\
0.0021 \\
4.0133 \\
0.0000 \\
14 \\
\end{array}$ & & \begin{tabular}{|l|} 
Consens \\
Consens \\
Maximum \\
Minimum \\
$\mathrm{N}$ \\
\end{tabular} & $\begin{array}{l}\text { s Mean } \\
\text { s Standard }\end{array}$ & Deviation & $\begin{array}{c}0.0133 \\
0.0039 \\
8.4067 \\
0.0000 \\
19 \\
\end{array}$ & & \begin{tabular}{|l|} 
Consensu \\
Consensu \\
Maximum \\
Minimum \\
$\mathrm{N}$ \\
\end{tabular} & $\begin{array}{l}\text { Mean } \\
\text { Stmdard }\end{array}$ & Deviation & $\begin{array}{c}0.0016 \\
0.0049 \\
0.0033 \\
0.0000 \\
1 \\
\end{array}$ & \\
\hline
\end{tabular}




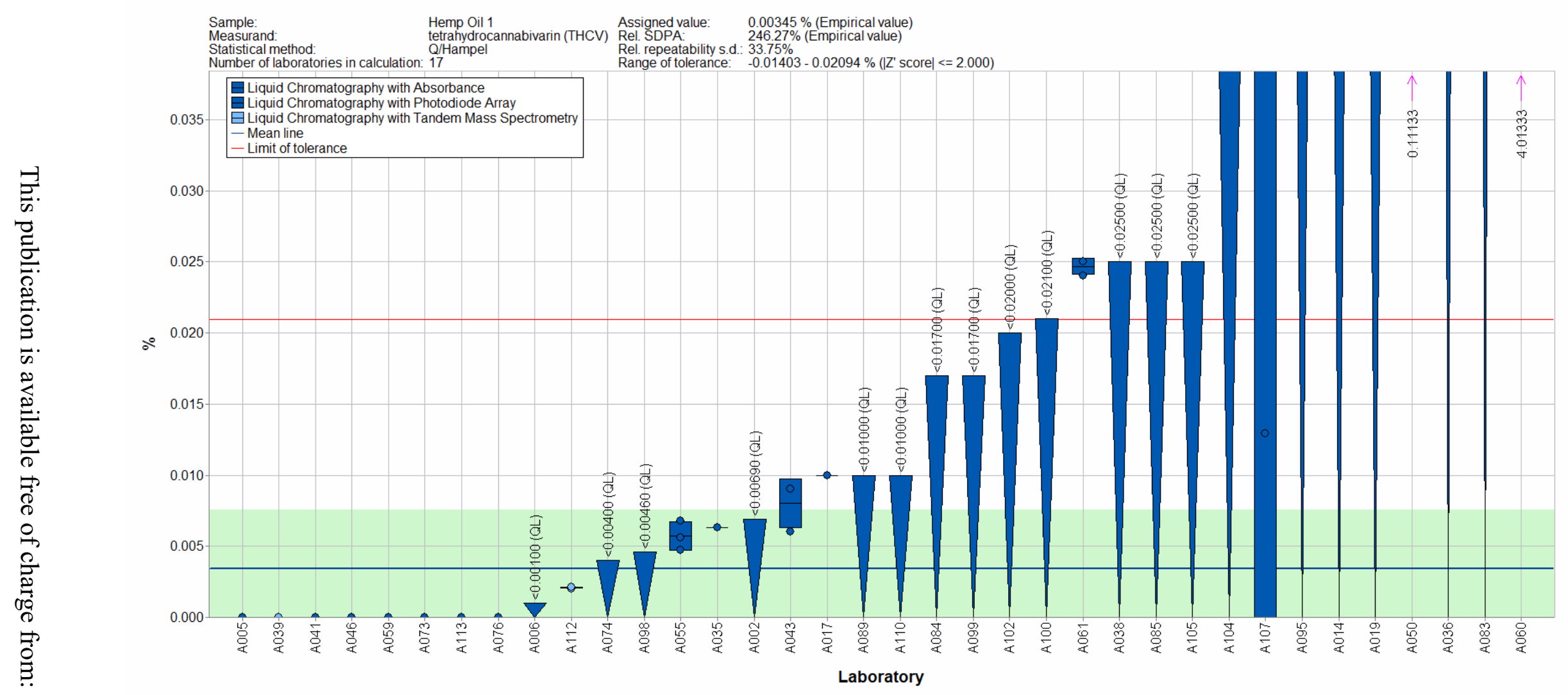

Figure 9-1. THCV in Hemp Oil 1 (data summary view - analytical method). In this view, individual laboratory data are plotted (circles) with the individual laboratory standard deviation (rectangle). The color of the data point represents the analytical method employed. The solid blue line represents the consensus mean, and the green shaded region represents the $95 \%$ confidence interval for the consensus mean. The solid red line represents the upper consensus range of tolerance, calculated as the values above the consensus mean that results in an acceptable $Z_{\text {comm }}^{\prime}$ score, $\left|Z_{\text {comm }}^{\prime}\right| \leq 2$, with the lower limit set at zero. A NIST value has not been determined in this material. The downward triangle represents data reported as a threshold or LOQ value. 


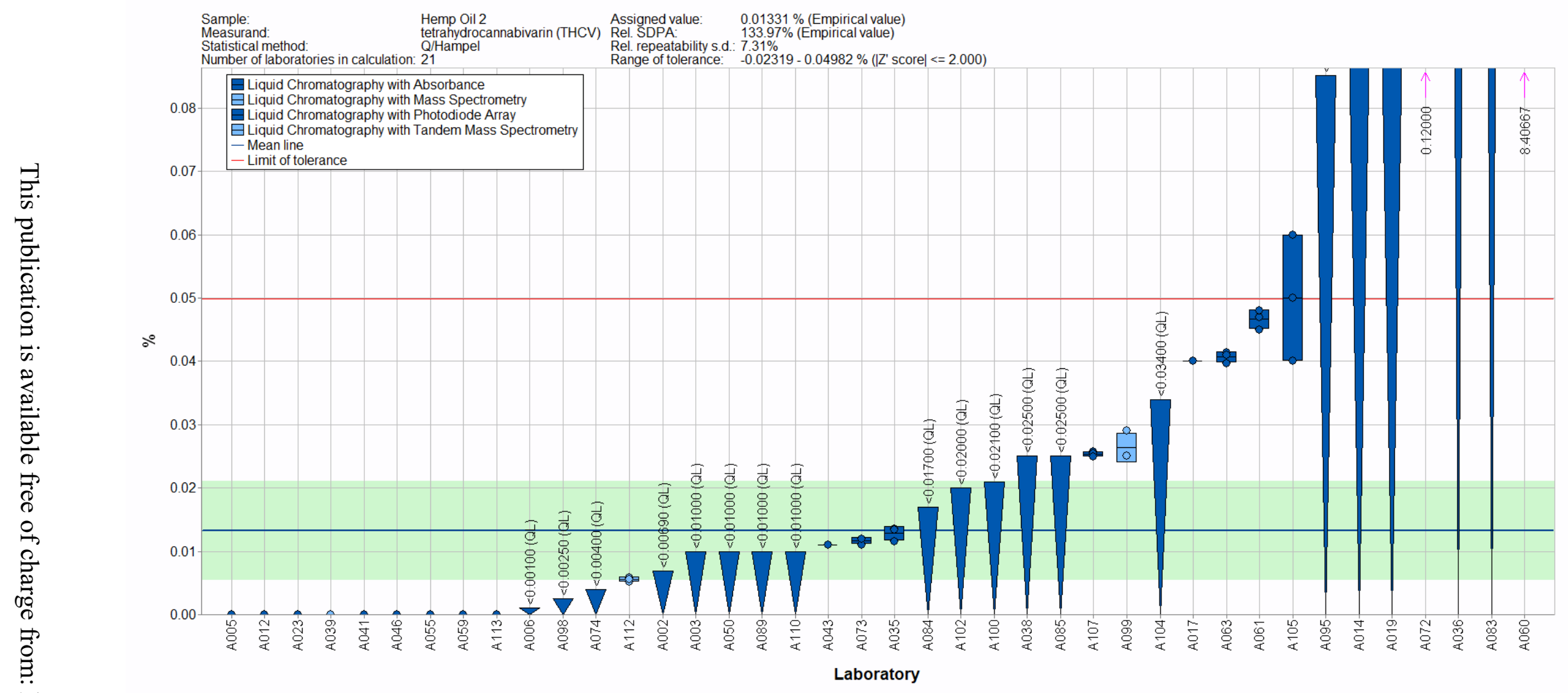

Figure 9-2. THCV in Hemp Oil 2 (data summary view - analytical method). In this view, individual laboratory data are plotted (circles) with the individual laboratory standard deviation (rectangle). The color of the data point represents the analytical method employed. The solid blue line represents the consensus mean, and the green shaded region represents the $95 \%$ confidence interval for the consensus mean. The solid red line represents the upper consensus range of tolerance, calculated as the values above the consensus mean that results in an acceptable $Z_{\text {comm }}^{\prime}$ score, $\left|Z_{\text {comm }}^{\prime}\right| \leq 2$, with the lower limit set at zero. A NIST value has not been determined in this material. The downward triangle represents data reported as a threshold or LOQ value. 


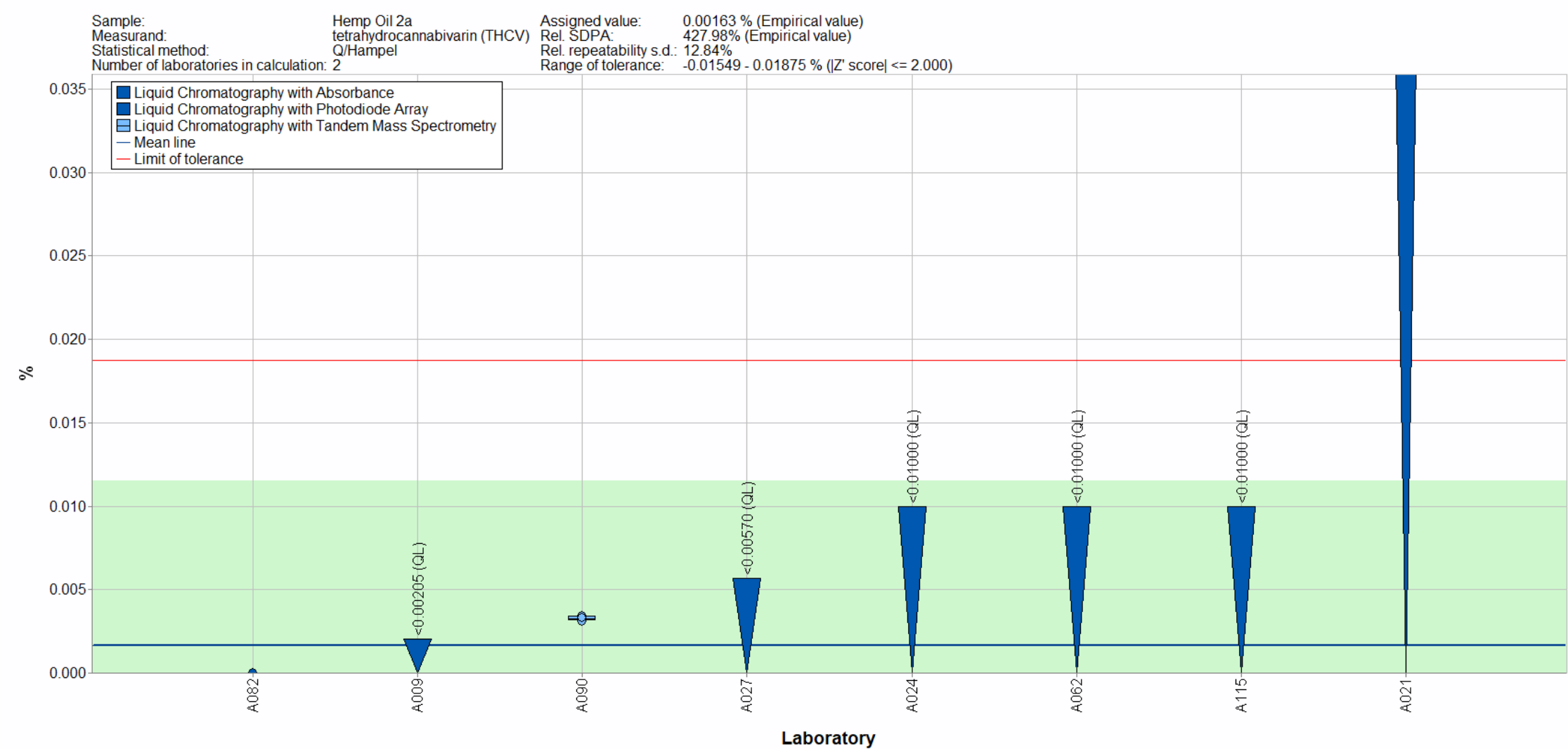

Figure 9-3. THCV in Hemp Oil 2a (data summary view - analytical method). In this view, individual laboratory data are plotted (circles) with the individual laboratory standard deviation (rectangle). The color of the data point represents the analytical method employed. The solid blue line represents the consensus mean, and the green shaded region represents the $95 \%$ confidence interval for the consensus mean. The solid red line represents the upper consensus range of tolerance, calculated as the values above the consensus mean that results in an acceptable $Z_{\text {comm }}^{\prime}$ score, $\left|Z_{\text {comm }}^{\prime}\right| \leq 2$, with the lower limit set at zero. A NIST value has not been determined in this material. The downward triangle represents data reported as a threshold or LOQ value. 


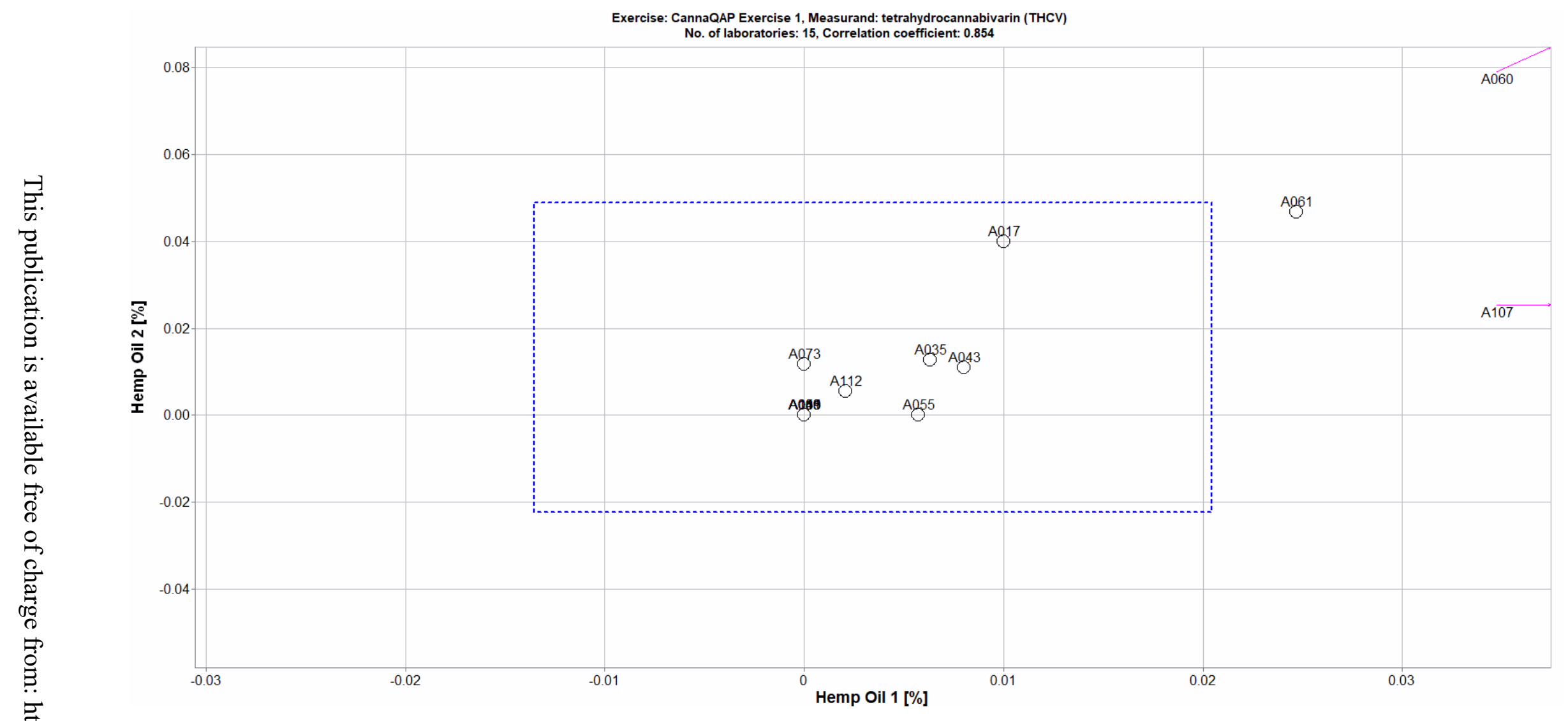

Figure 9-4. Laboratory means for THCV in Hemp Oil 1 and Hemp Oil 2 (sample/sample comparison view). In this view, the individual laboratory mean for one sample (Hemp Oil 1) is compared to the individual laboratory mean for a second sample (Hemp Oil 2). The dotted blue box represents the consensus range of tolerance for Hemp Oil 1 (x-axis) and Hemp Oil 2 (y-axis), calculated as the values above and below the consensus means that result in an acceptable $Z_{\text {comm }}^{\prime}$ score, $\left|Z_{\text {comm }}^{\prime}\right| \leq 2$. 
Table 9-3. Data summary table for THCVA in hemp oils. Data highlighted in red have been flagged as a data entry of zero or results that include text (e.g., "<LOQ" or "present").

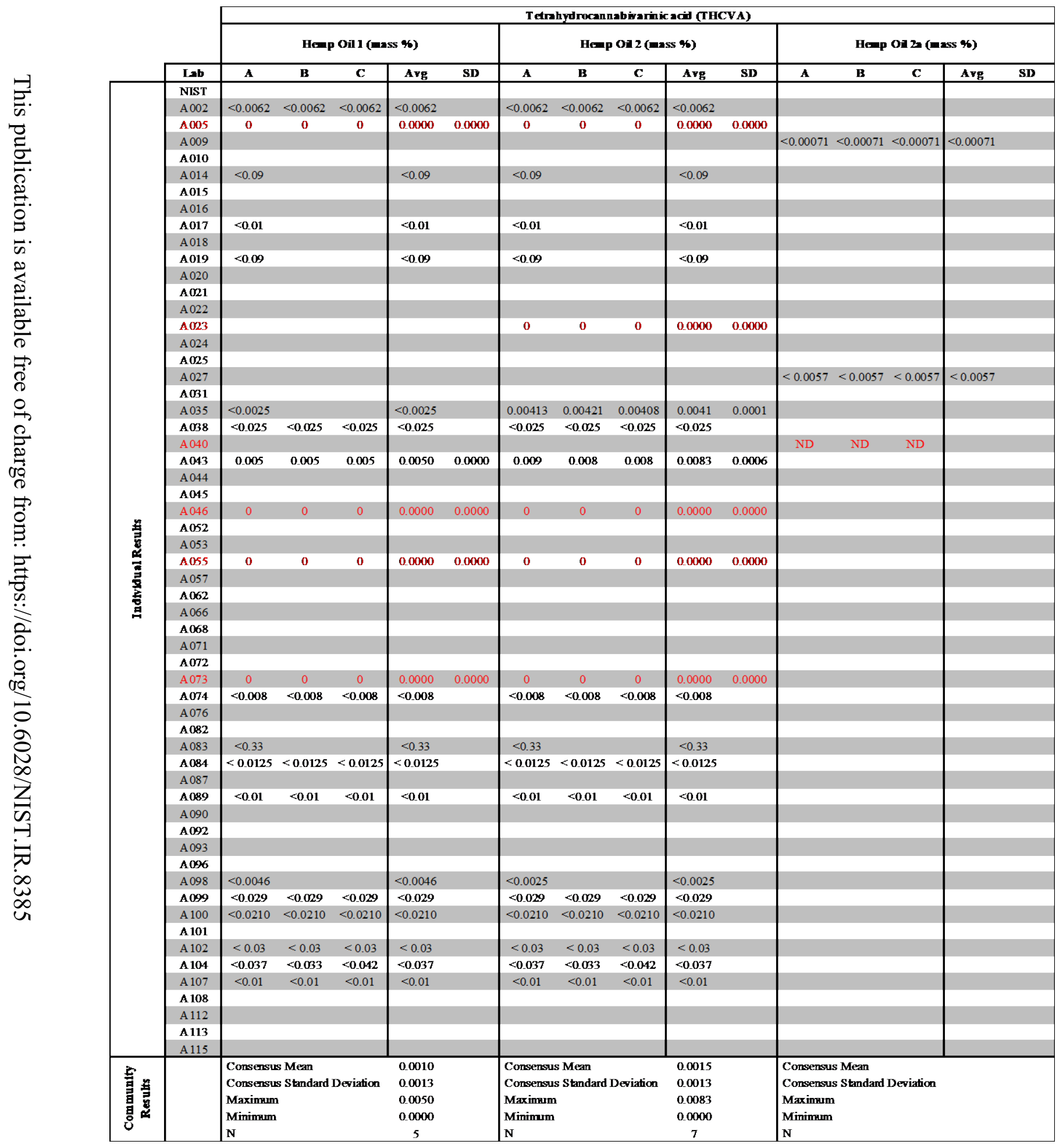




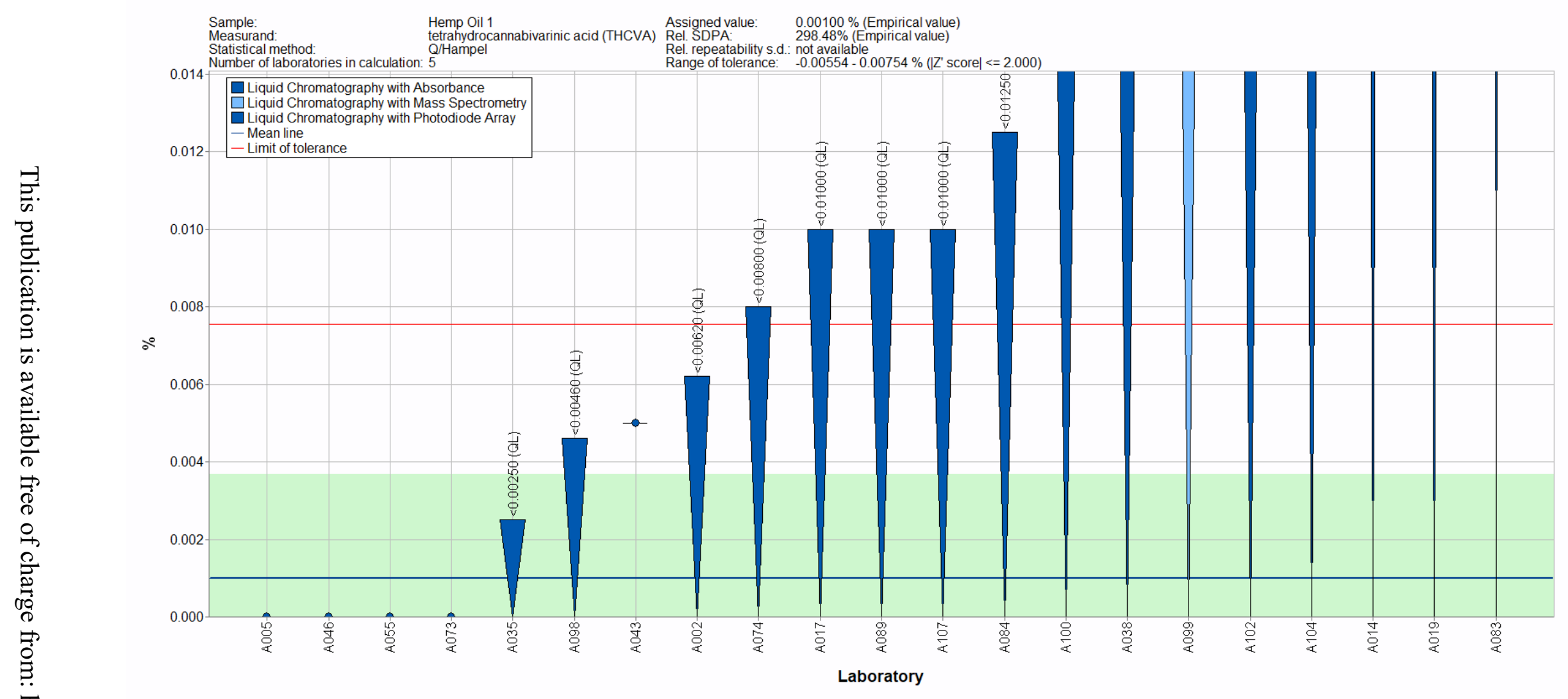

Figure 9-5. THCVA in Hemp Oil 1 (data summary view - analytical method). In this view, individual laboratory data are plotted (circles) with the individual laboratory standard deviation (rectangle). The color of the data point represents the analytical method employed. The solid blue line represents the consensus mean, and the green shaded region represents the $95 \%$ confidence interval for the consensus mean. The solid red line represents the upper consensus range of tolerance, calculated as the values above the consensus mean that results in an acceptable $Z_{\text {comm }}^{\prime}$ score, $\left|Z_{\text {comm }}^{\prime}\right| \leq 2$, with the lower limit set at zero. A NIST value has not been determined in this material. The downward triangle represents data reported as a threshold or LOQ value. 


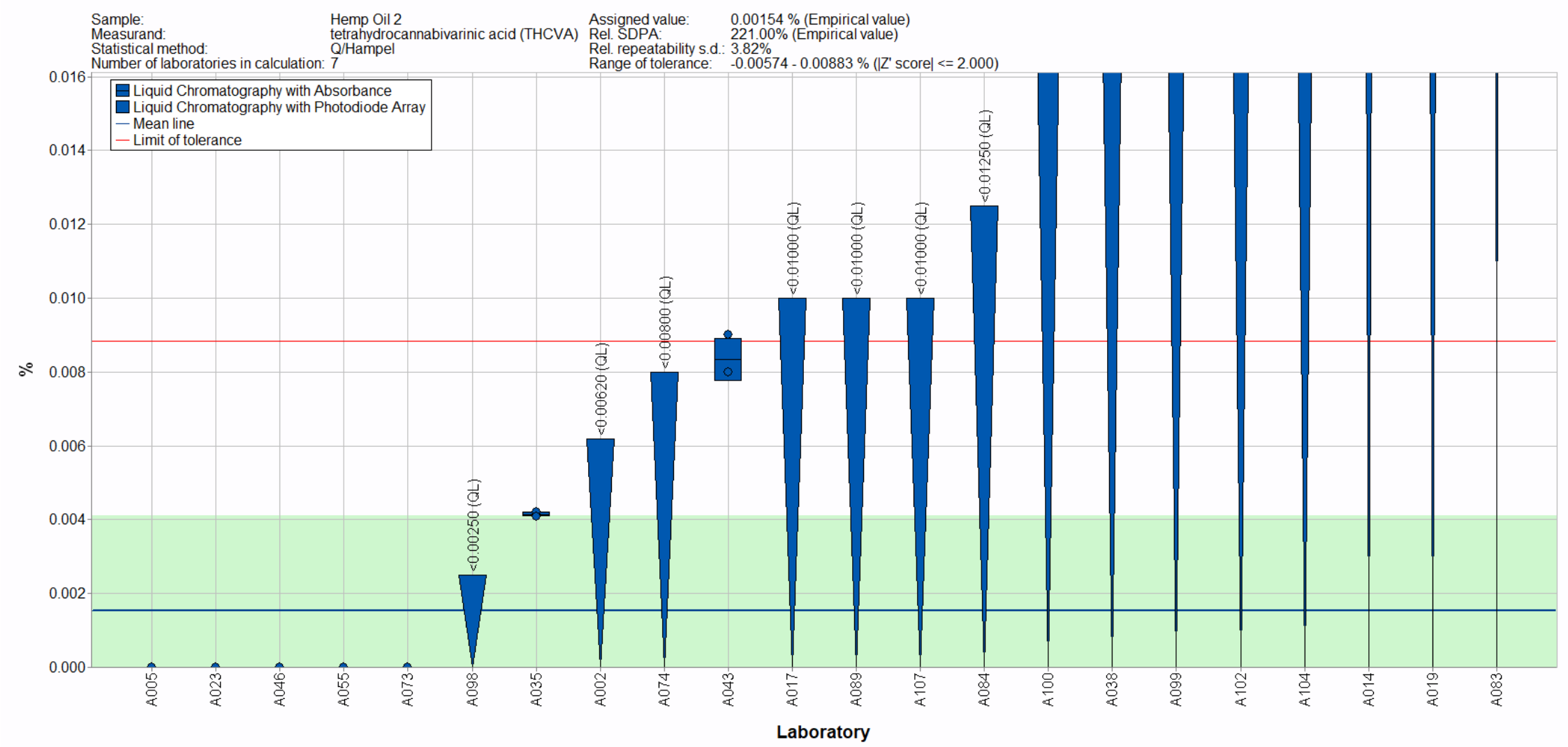

Figure 9-6. THCVA in Hemp Oil 2 (data summary view - analytical method). In this view, individual laboratory data are plotted (circles) with the individual laboratory standard deviation (rectangle). The color of the data point represents the analytical method employed. The solid blue line represents the consensus mean, and the green shaded region represents the $95 \%$ confidence interval for the consensus mean. The solid red line represents the upper consensus range of tolerance, calculated as the values above the consensus mean that results in an acceptable $Z_{\text {comm }}^{\prime}$ score, $\left|Z_{\text {comm }}^{\prime}\right| \leq 2$, with the lower limit set at zero. A NIST value has not been determined in this material. The downward triangle represents data reported as a threshold or LOQ value. 


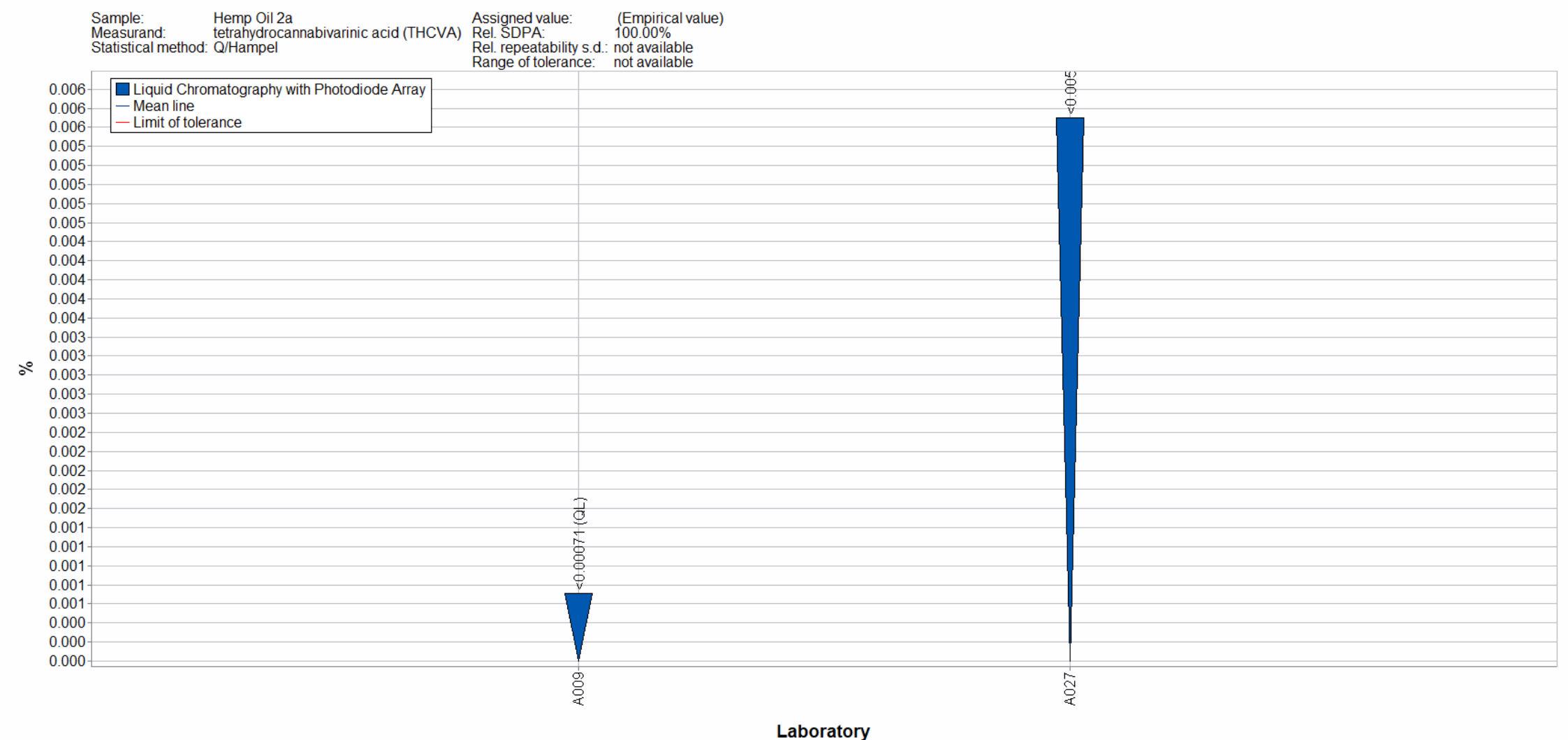

Figure 9-7. THCVA in Hemp Oil 2a (data summary view - analytical method). In this view, individual laboratory data are plotted (circles) with the individual laboratory standard deviation (rectangle). The color of the data point represents the analytical method employed. The downward triangle represents data reported as a threshold or LOQ value. 


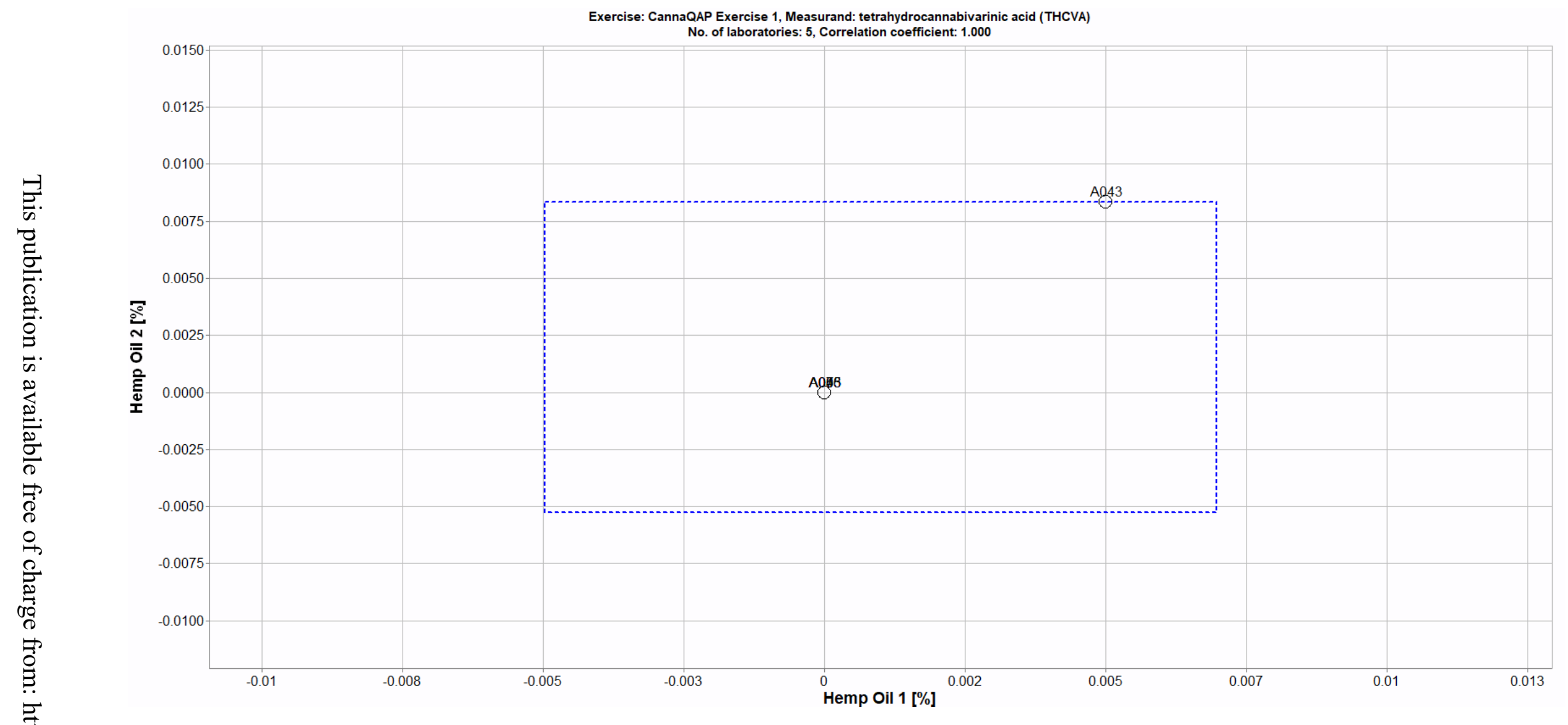

Figure 9-8. Laboratory means for THCVA in Hemp Oil 1 and Hemp Oil 2 (sample/sample comparison view). In this view, the individual laboratory mean for one sample (Hemp Oil 1) is compared to the individual laboratory mean for a second sample (Hemp Oil 2). The dotted blue box represents the consensus range of tolerance for Hemp Oil 1 (x-axis) and Hemp Oil 2 (y-axis), calculated as the values above and below the consensus means that result in an acceptable $Z_{\text {comm }}^{\prime}$ score, $\left|Z_{\text {comm }}^{\prime}\right| \leq 2$. 


\section{Appendix I - Method Questionnaire Responses}

41 laboratories completed the method questionnaire.

\begin{tabular}{l|l|l|l|l|l|l} 
A002 & A013 & A028 & A046 & A060 & A083 & A100 \\
A005 & A015 & A031 & A050 & A062 & A085 & A102 \\
A007 & A020 & A033 & A052 & A063 & A088 & A104 \\
A008 & A021 & A038 & A054 & A071 & A089 & A111 \\
A009 & A024 & A039 & A055 & A072 & A092 & A114 \\
A012 & A026 & A041 & A059 & A081 & A093 &
\end{tabular}

\section{Sample Preparation}

All laboratories that tested both samples (Hemp Oil 1 and Hemp Oil 2) reported using the same sample preparation approach for both samples.

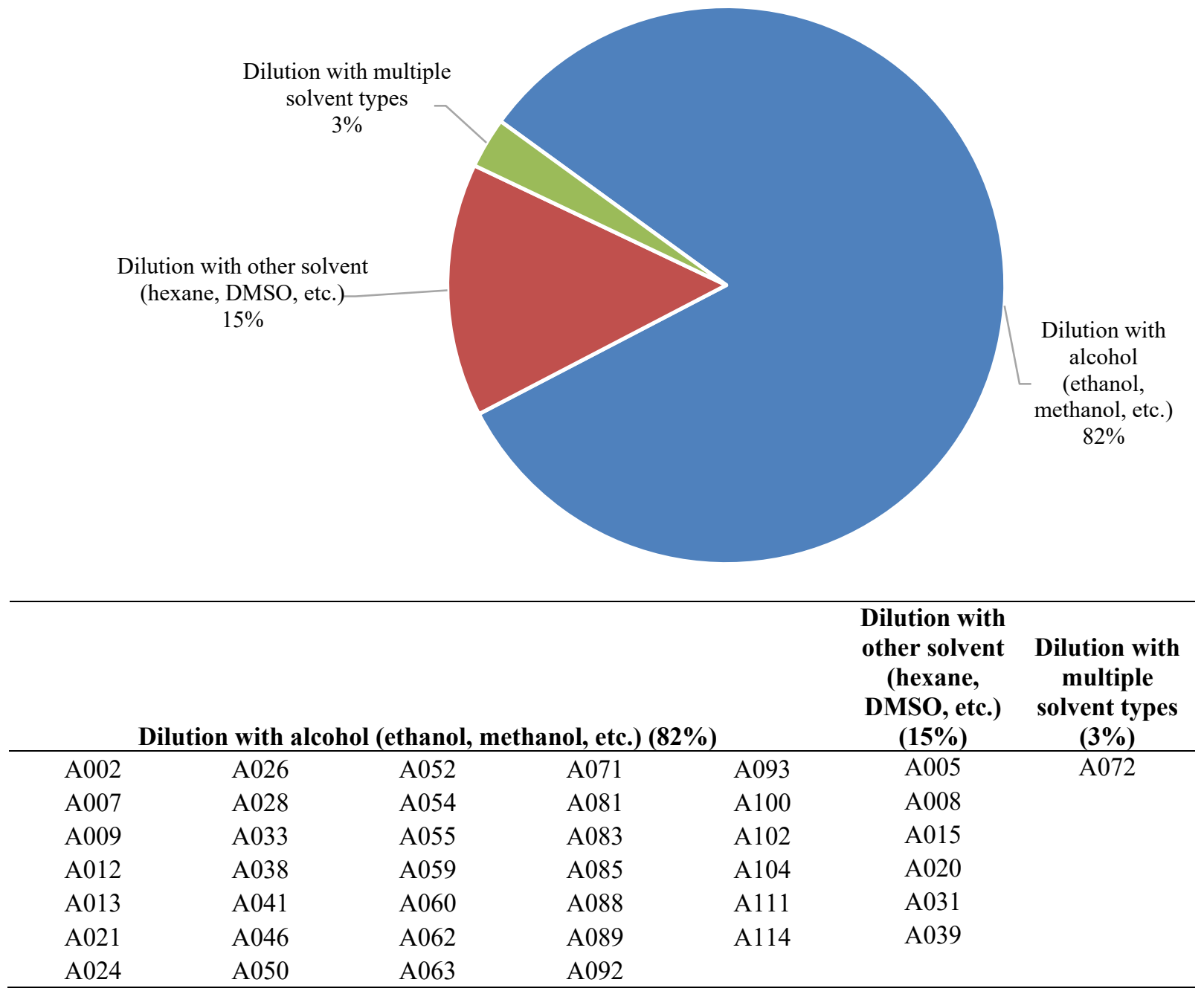




\section{General Analytical Methods}

All laboratories that tested both samples (Hemp Oil 1 and Hemp Oil 2) reported using the same analytical methods for both samples.

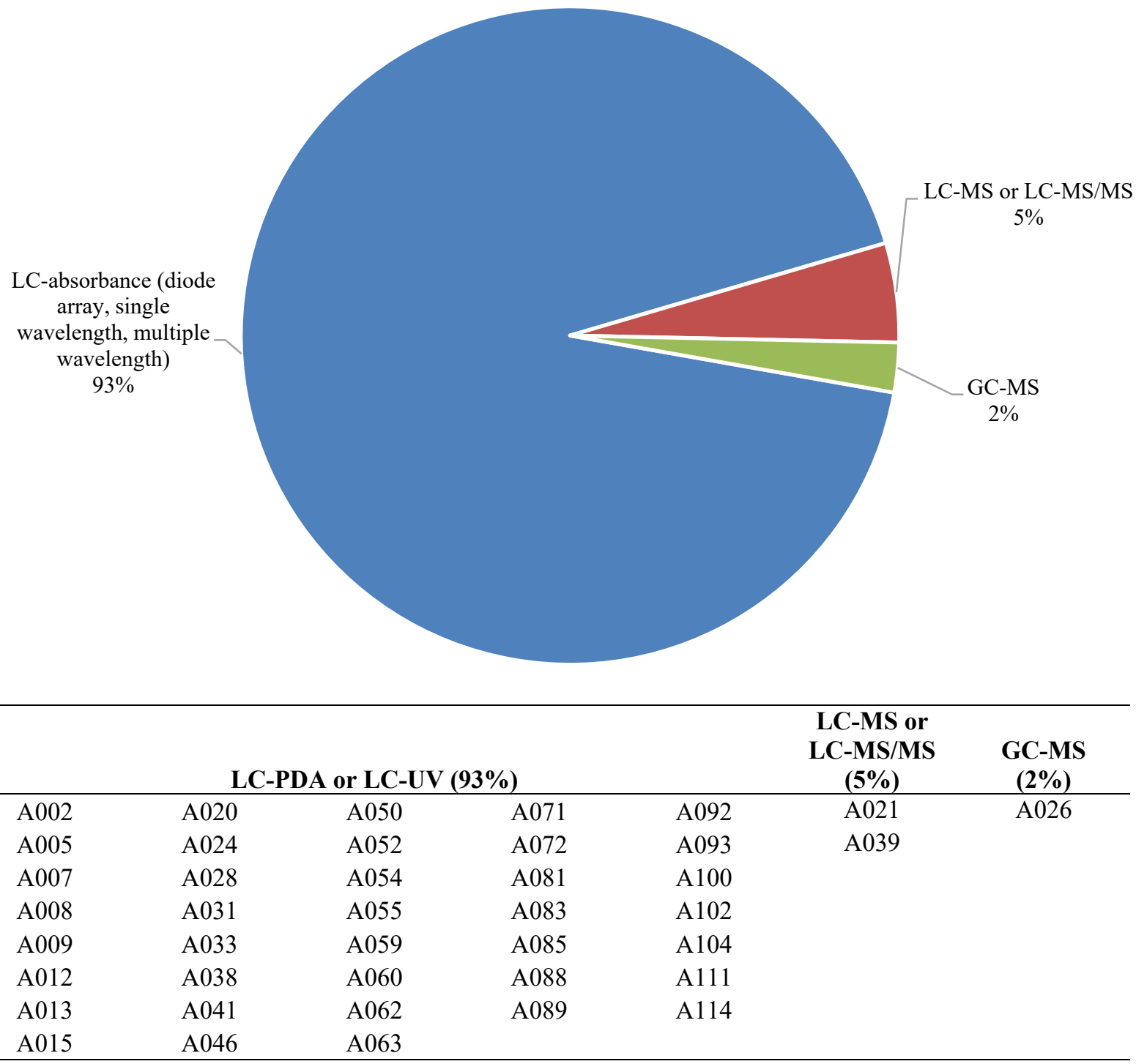




\section{LC-PDA and LC-UV Method Information}

\section{Injection Conditions}

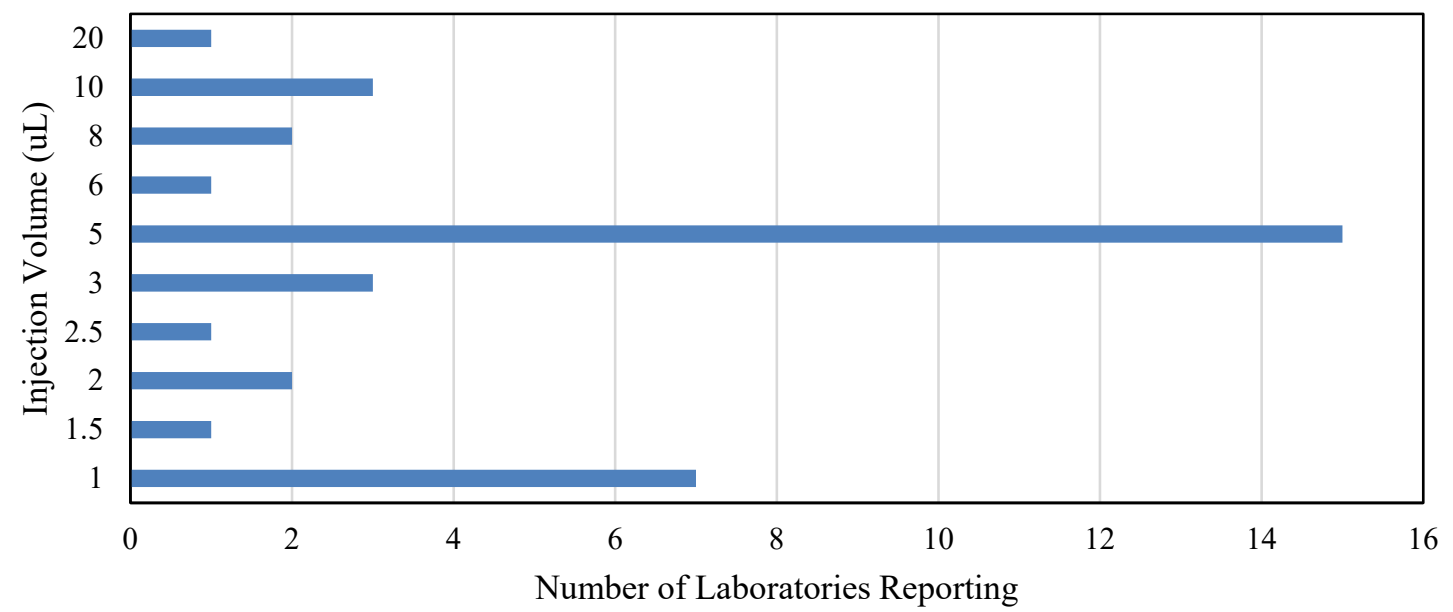

\begin{tabular}{|c|c|c|c|c|c|c|c|c|}
\hline $1 \mu \mathrm{L}(19 \%)$ & A005 & A008 & A033 & A052 & A055 & A059 & A104 & \\
\hline $1.5 \mu \mathrm{L}(3 \%)$ & A089 & & & & & & & \\
\hline $2 \mu \mathrm{L}(6 \%)$ & A038 & A114 & & & & & & \\
\hline $2.5 \mu \mathrm{L}(3 \%)$ & A072 & & & & & & & \\
\hline $3 \mu \mathrm{L}(8 \%)$ & A009 & A013 & A050 & & & & & \\
\hline \multirow[t]{2}{*}{$5 \mu \mathrm{L}(42 \%)$} & A002 & A007 & A012 & A015 & A024 & A046 & A054 & A060 \\
\hline & A063 & A081 & A085 & A088 & A093 & A 100 & A111 & \\
\hline $6 \mu \mathrm{L}(3 \%)$ & $\mathrm{A} 031$ & & & & & & & \\
\hline $8 \mu \mathrm{L}(6 \%)$ & A041 & A102 & & & & & & \\
\hline $10 \mu \mathrm{L}(8 \%)$ & A028 & A083 & A092 & & & & & \\
\hline $20 \mu \mathrm{L}(3 \%)$ & A071 & & & & & & & \\
\hline
\end{tabular}


Use of Wash Solvent

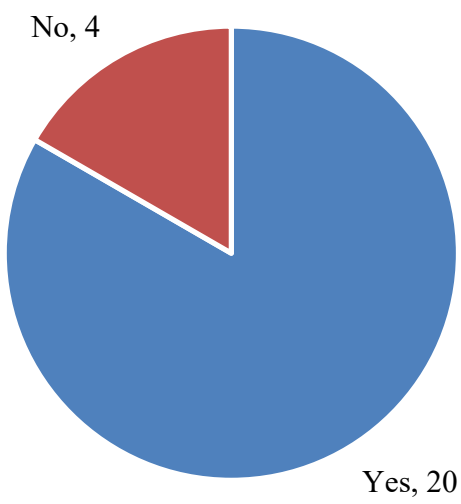

Type of Wash Solvent

* of the 20 participants reporting use of a solvent wash

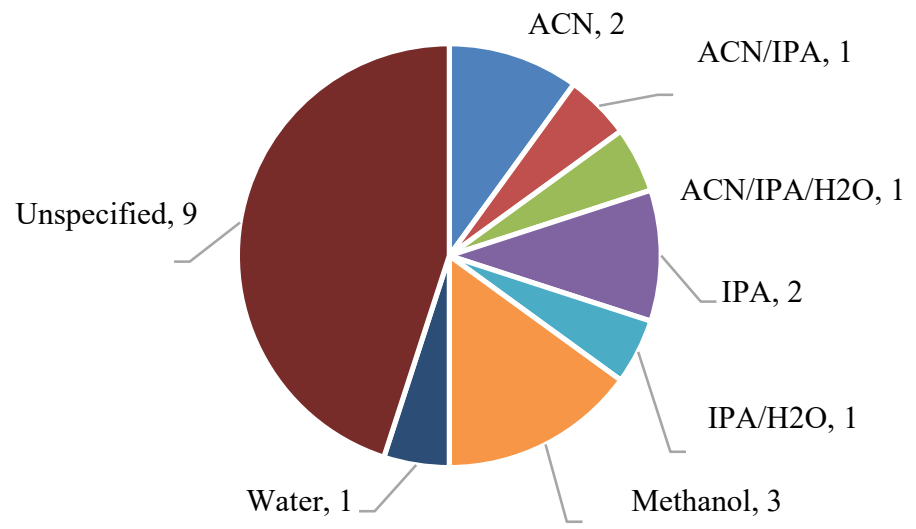

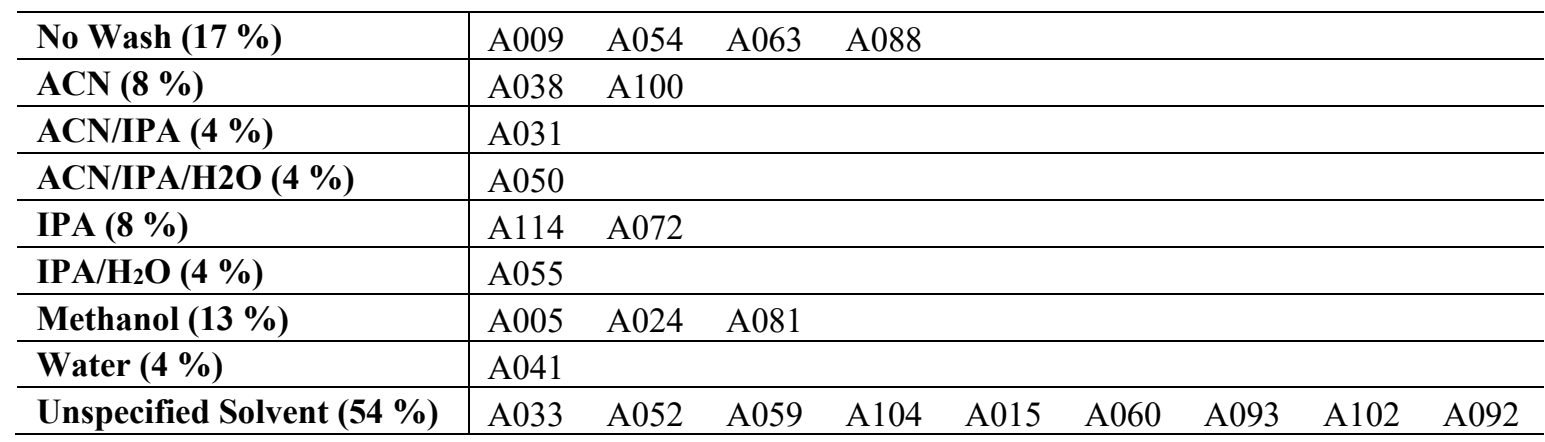


Column Type

All laboratories reported using a $\mathrm{C}_{18}$ column.

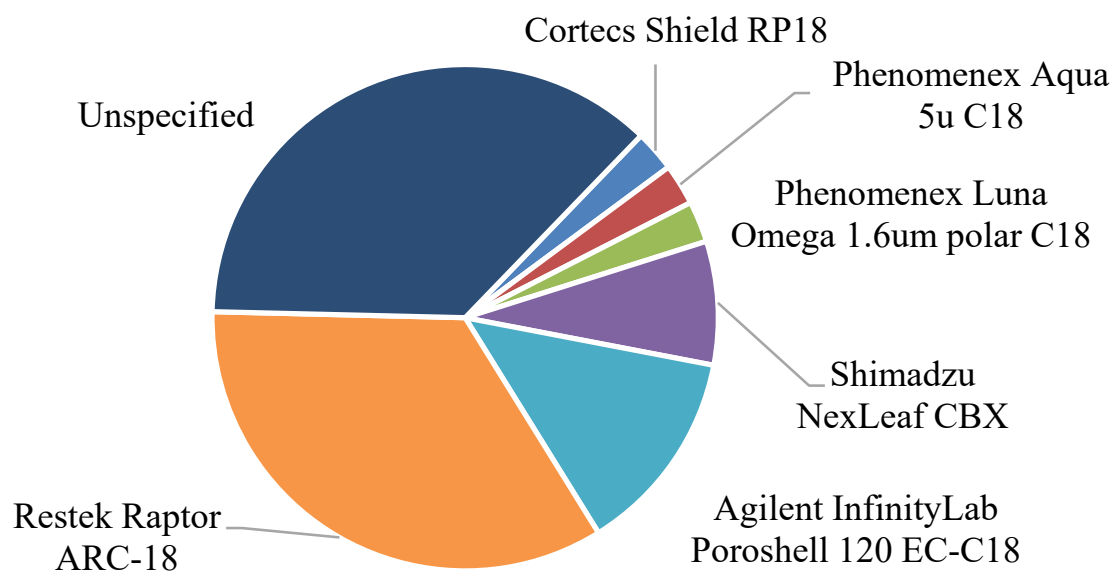

\begin{tabular}{l|lllllll}
\hline Cortecs Shield RP18 (3 \%) & A028 & & & & & \\
\hline Phenomenex Aqua 5u C18 (3 \%) & A071 & & & & & & \\
\hline Phenomenex Luna Omega 1.6um polar C18 (3 \%) & A033 & & & & & & \\
\hline Shimadzu NexLeaf CBX (8 \%) & A012 & A062 & A111 & & & & \\
\hline Agilent InfinityLab Poroshell 120 EC-C18 (13 \%) & A054 & A063 & A081 & A102 & A013 & & \\
\hline Restek Raptor ARC-18 (34 \%) & A007 & A038 & A052 & A055 & A088 & A093 & A104 \\
& A085 & A031 & A041 & A089 & A009 & A015 & \\
\hline Unspecified C18 Column (37 \%) & A020 & A002 & A046 & A092 & A024 & A100 & A114 \\
& A050 & A083 & A008 & A072 & A005 & A059 & A060 \\
\hline
\end{tabular}




\section{Column Temperature}

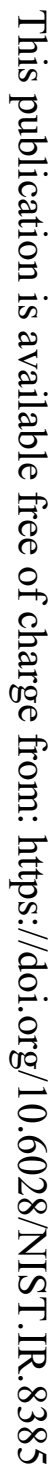

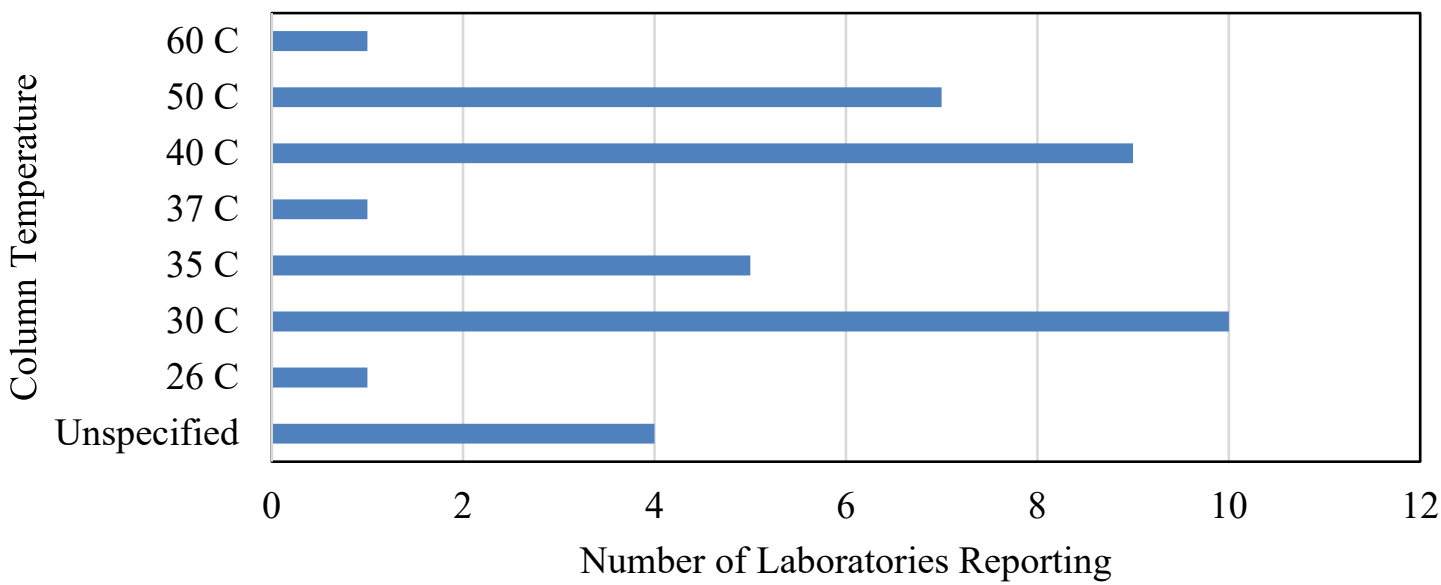

\begin{tabular}{|c|c|c|c|c|c|c|c|c|c|c|}
\hline $26^{\circ} \mathrm{C}(3 \%)$ & $\mathrm{A} 020$ & & & & & & & & & \\
\hline $30{ }^{\circ} \mathrm{C}(26 \%)$ & A002 & A007 & A038 & A046 & A052 & A055 & A088 & A092 & A093 & A104 \\
\hline $35^{\circ} \mathrm{C}(13 \%)$ & $\mathrm{A} 012$ & A024 & A062 & A100 & A114 & & & & & \\
\hline $37^{\circ} \mathrm{C}(3 \%)$ & $\mathrm{A} 085$ & & & & & & & & & \\
\hline $40^{\circ} \mathrm{C}(24 \%)$ & $\mathrm{A} 028$ & A031 & A033 & A041 & $\mathrm{A} 050$ & A071 & A083 & A089 & A111 & \\
\hline $50{ }^{\circ} \mathrm{C}(18 \%)$ & A008 & A009 & A015 & $\mathrm{A} 054$ & A063 & A081 & A 102 & & & \\
\hline $60^{\circ} \mathrm{C}(3 \%)$ & A072 & & & & & & & & & \\
\hline Unspecified Temperature (11 \%) & $\mathrm{A} 005$ & $\mathrm{~A} 013$ & A059 & A060 & & & & & & \\
\hline
\end{tabular}


Mobile Phase

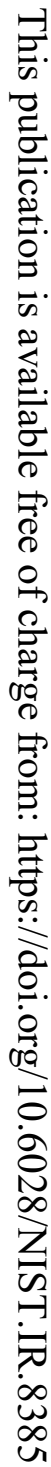

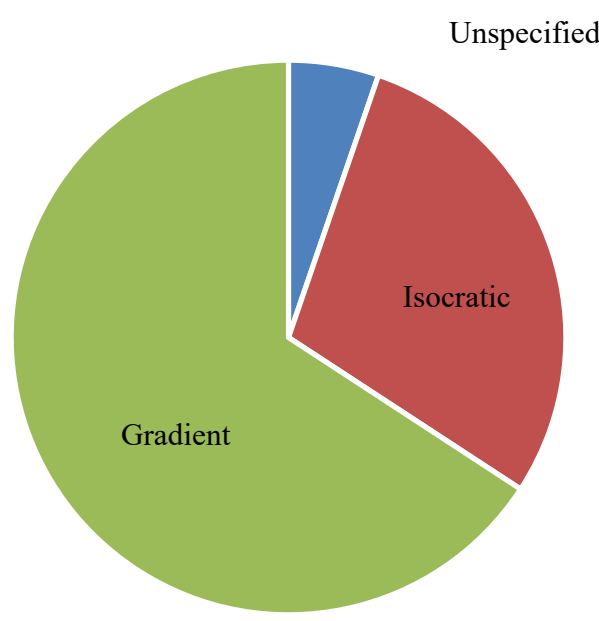

\begin{tabular}{|c|c|c|c|c|c|c|c|c|c|c|}
\hline Isocratic Separation (29 \%) & $\begin{array}{l}\text { A007 } \\
\text { A046 } \\
\text { A081 }\end{array}$ & $\begin{array}{l}\text { A008 } \\
\text { A050 } \\
\text { A092 }\end{array}$ & $\begin{array}{l}\text { A009 } \\
\text { A052 } \\
\text { A100 } \\
\end{array}$ & $\begin{array}{l}\mathrm{A} 012 \\
\mathrm{~A} 054 \\
\mathrm{~A} 102 \\
\end{array}$ & $\begin{array}{l}\text { A013 } \\
\text { A059 } \\
\text { A114 } \\
\end{array}$ & $\begin{array}{l}\text { A015 } \\
\text { A060 }\end{array}$ & $\begin{array}{l}\text { A020 } \\
\text { A062 }\end{array}$ & $\begin{array}{l}\text { A024 } \\
\text { A063 }\end{array}$ & $\begin{array}{l}\text { A028 } \\
\text { A071 }\end{array}$ & $\begin{array}{l}\text { A038 } \\
\text { A072 }\end{array}$ \\
\hline Gradient Separation (66 \%) & $\begin{array}{l}\text { A002 } \\
\text { A111 } \\
\end{array}$ & A033 & A041 & A055 & A083 & A085 & A088 & A089 & A093 & A 104 \\
\hline Unspecified Separation Type (5\%) & $\mathrm{A} 005$ & A031 & & & & & & & & \\
\hline
\end{tabular}

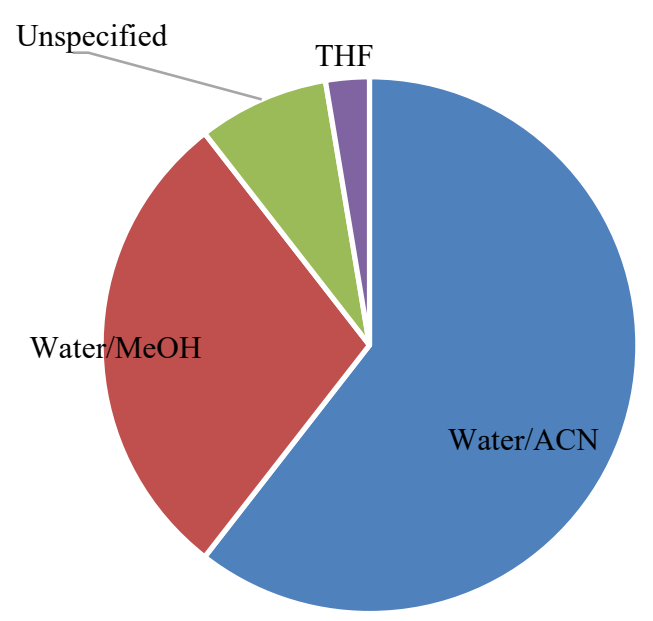

\begin{tabular}{|c|c|c|c|c|c|c|c|c|c|c|}
\hline Water/ACN (61 \%) & $\begin{array}{l}\text { A002 } \\
\text { A060 } \\
\text { A114 } \\
\end{array}$ & $\begin{array}{l}\text { A012 } \\
\text { A083 }\end{array}$ & $\begin{array}{l}\text { A024 } \\
\text { A085 }\end{array}$ & $\begin{array}{l}\text { A028 } \\
\text { A088 }\end{array}$ & $\begin{array}{l}\text { A033 } \\
\text { A089 }\end{array}$ & $\begin{array}{l}\text { A038 } \\
\text { A092 }\end{array}$ & $\begin{array}{l}\text { A041 } \\
\text { A093 }\end{array}$ & $\begin{array}{l}\text { A046 } \\
\text { A100 }\end{array}$ & $\begin{array}{l}\text { A050 } \\
\text { A104 }\end{array}$ & $\begin{array}{l}\text { A052 } \\
\text { A111 }\end{array}$ \\
\hline Water/MeOH (29\%) & $\begin{array}{l}\text { A008 } \\
\text { A102 }\end{array}$ & A009 & A013 & A020 & A054 & A059 & A063 & A071 & A072 & A081 \\
\hline THF (8\%) & A031 & & & & & & & & & \\
\hline Unspecified Solvent (8 \%) & A005 & A015 & A062 & & & & & & & \\
\hline
\end{tabular}




\section{Modifiers}

星.
0
0
0
0
0
0
0
0
0
0
0
0
0
0
0
0
0
0
0
0
0
0
00
0
0
0
0
0
0
0
0
0
0
0
0
00
0
0
0
0
0
0
0
0
0
0
0
0
0
0

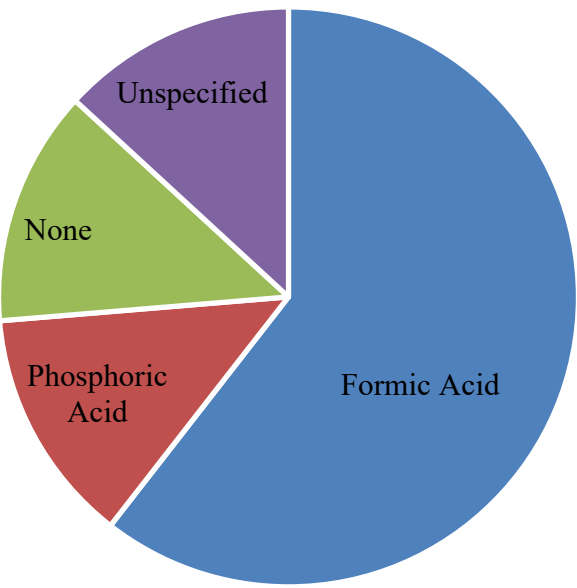

\begin{tabular}{|c|c|c|c|c|c|c|c|c|c|c|}
\hline Formic Acid (61 \%) & $\begin{array}{l}\text { A008 } \\
\text { A054 } \\
\text { A093 }\end{array}$ & $\begin{array}{l}\text { A009 } \\
\text { A055 } \\
\text { A104 }\end{array}$ & $\begin{array}{l}\text { A028 } \\
\text { A059 } \\
\text { A114 }\end{array}$ & $\begin{array}{l}\text { A031 } \\
\text { A063 }\end{array}$ & $\begin{array}{l}\text { A033 } \\
\text { A072 }\end{array}$ & $\begin{array}{l}\text { A038 } \\
\text { A081 }\end{array}$ & $\begin{array}{l}\text { A041 } \\
\text { A083 }\end{array}$ & $\begin{array}{l}\text { A046 } \\
\text { A085 }\end{array}$ & $\begin{array}{l}\text { A050 } \\
\text { A088 }\end{array}$ & $\begin{array}{l}\text { A052 } \\
\text { A092 }\end{array}$ \\
\hline Phosphoric Acid (13 \%) & A007 & A060 & $\mathrm{A} 071$ & $\mathrm{~A} 100$ & A111 & & & & & \\
\hline No Modifier (13 \%) & $\mathrm{A} 002$ & $\mathrm{~A} 012$ & $\mathrm{~A} 013$ & $\mathrm{~A} 020$ & A089 & & & & & \\
\hline Unspecified Modifier (13\%) & A005 & A015 & A024 & A062 & A102 & & & & & \\
\hline
\end{tabular}




\section{Detector Wavelength}

*note some laboratories reported using multiple distinct wavelengths (up to 4)

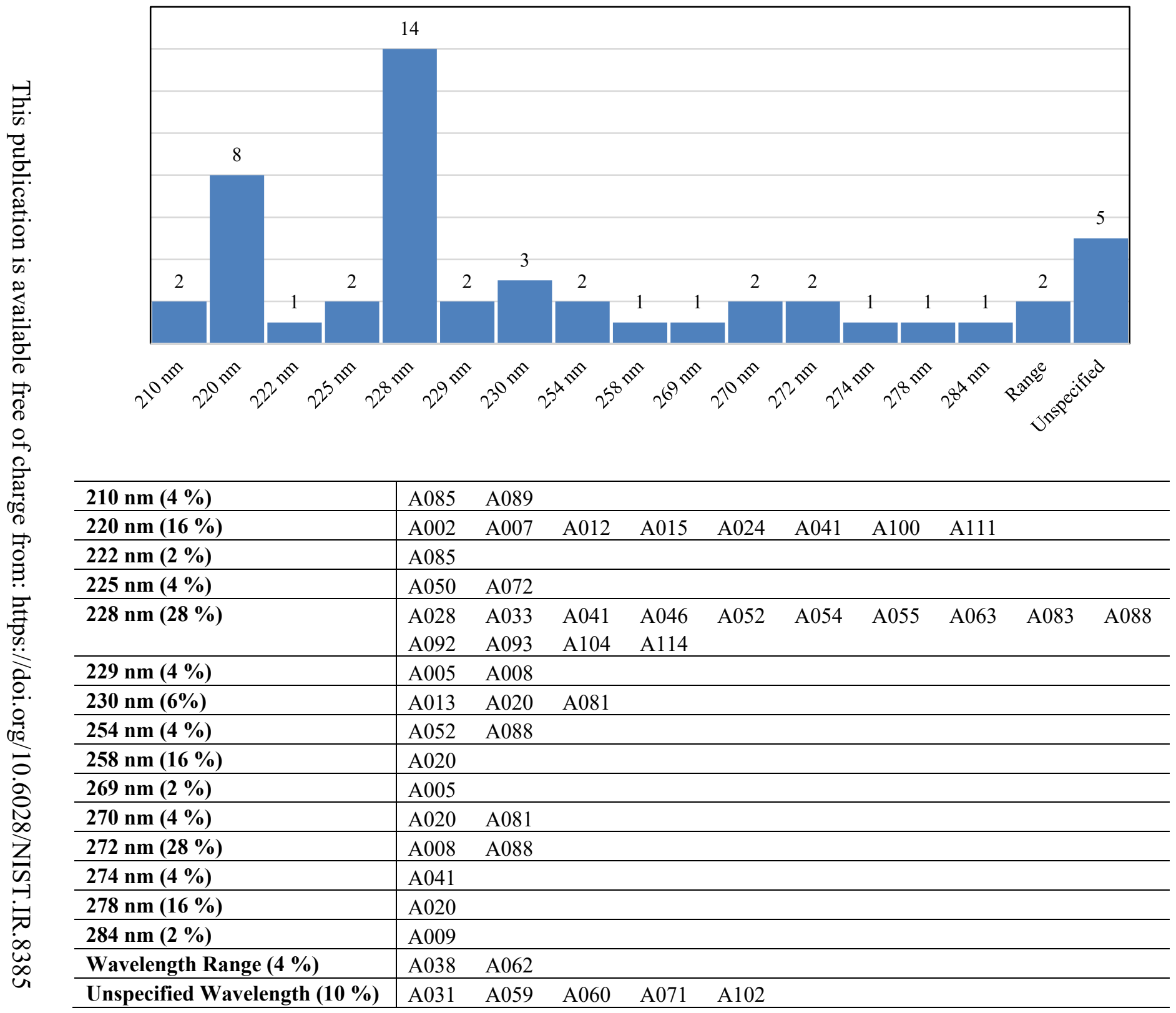




\section{Detector Type}

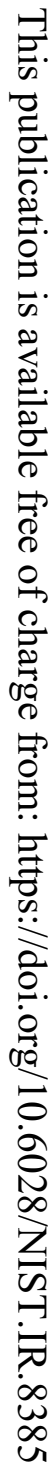

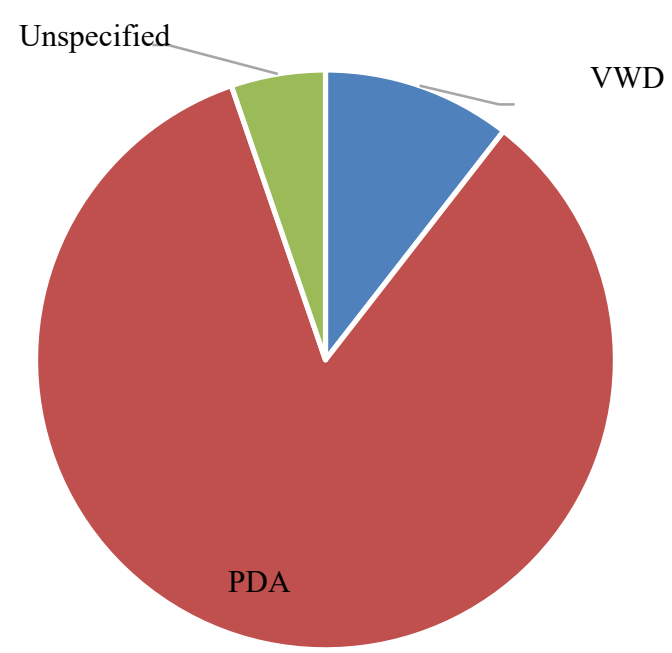

\begin{tabular}{l|llllllllll}
\hline PDA (84 \%) & A085 & A002 & A012 & A015 & A024 & A041 & A100 & A050 & A072 & A028 \\
& A033 & A046 & A052 & A054 & A055 & A063 & A088 & A092 & A104 & A114 \\
& A005 & A013 & A020 & A081 & A008 & A009 & A038 & A062 & A031 & A059 \\
& A071 & A102 & & & & & & & & \\
\hline VWD (11 \%) & A089 & A007 & A111 & A060 & & & & & & \\
\hline Unspecified Modifier (5 \%) & A083 & A093 & & & & & & & \\
\hline
\end{tabular}




\section{Calibration Type}

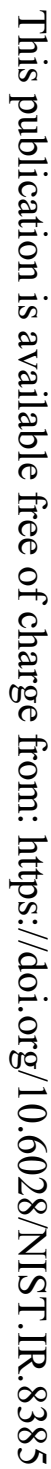

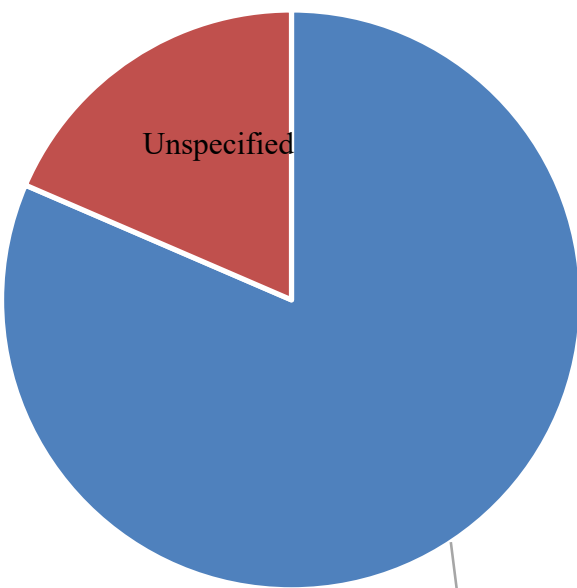

Curve

\begin{tabular}{l|llllllllll}
\hline Calibration Curve (76 \%) & A002 & A007 & A008 & A009 & A015 & A024 & A028 & A031 & A038 & A041 \\
& A050 & A052 & A054 & A055 & A062 & A063 & A072 & A081 & A083 & A085 \\
& A088 & A089 & A092 & A093 & A100 & A102 & A104 & A111 & A114 & \\
\hline Unspecified Approach (24 \%) & A005 & A012 & A013 & A020 & A033 & A046 & A059 & A060 & A071 \\
\hline
\end{tabular}

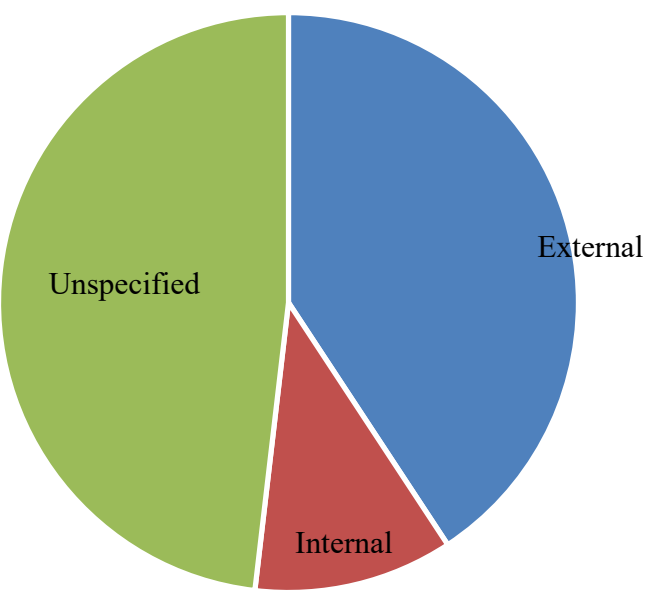

\begin{tabular}{l|llllllllll}
\hline External Standard (50 \%) & A002 & A008 & A013 & A020 & A028 & A033 & A038 & A050 & A055 & A059 \\
& A060 & A062 & A071 & A072 & A088 & A089 & A093 & A100 & A104 & \\
\hline Internal Standard (4 \%) & A009 & A046 & A052 & A114 & & & & & & \\
\hline Unspecified Approach (39 \%) & A005 & A007 & A012 & A015 & A024 & A031 & A041 & A054 & A063 & A081 \\
& A083 & A085 & A092 & A102 & A111 & & & & & \\
\hline
\end{tabular}




\section{Source of Calibrants}

*note that some laboratories reported use of standards from multiple providers

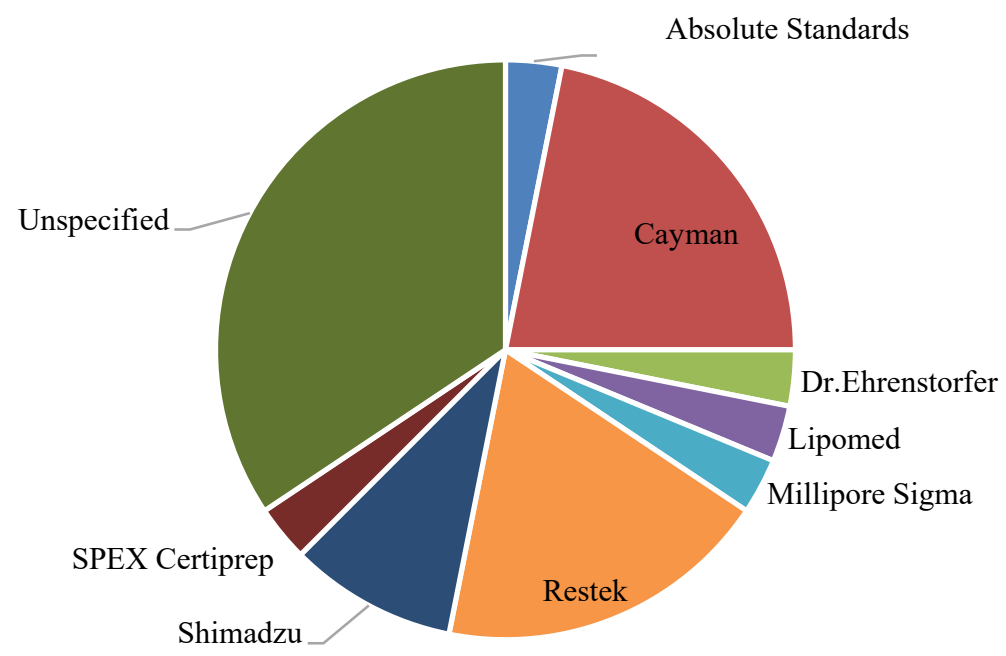

\begin{tabular}{|c|c|c|c|c|c|c|c|c|c|c|}
\hline Cerilliant (36 \%) & $\begin{array}{l}\text { A005 } \\
\text { A054 }\end{array}$ & $\begin{array}{l}\text { A008 } \\
\text { A055 }\end{array}$ & $\begin{array}{l}\text { A009 } \\
\text { A071 }\end{array}$ & $\begin{array}{l}\text { A015 } \\
\text { A081 }\end{array}$ & $\begin{array}{l}\text { A031 } \\
\text { A083 }\end{array}$ & $\begin{array}{l}\text { A033 } \\
\text { A100 }\end{array}$ & $\begin{array}{l}\text { A038 } \\
\text { A102 }\end{array}$ & $\begin{array}{l}\text { A041 } \\
\text { A104 }\end{array}$ & A046 & A052 \\
\hline Absolute Standards (2 \%) & A028 & & & & & & & & & \\
\hline Cayman (14\%) & A007 & A038 & A062 & A063 & A081 & A088 & A111 & & & \\
\hline Dr. Ehrenstorfer (2\%) & A054 & & & & & & & & & \\
\hline Lipomed (2 \%) & A102 & & & & & & & & & \\
\hline Millipore Sigma (2 \%) & A009 & & & & & & & & & \\
\hline Restek (12\%) & $\mathrm{A} 005$ & A038 & A041 & A072 & A081 & A085 & & & & \\
\hline Shimadzu (6 \%) & $\mathrm{A} 012$ & A062 & A100 & & & & & & & \\
\hline SPEX Certiprep (2\%) & A009 & & & & & & & & & \\
\hline Unspecified Approach (22 \%) & $\begin{array}{l}\text { A002 } \\
\text { A114 }\end{array}$ & A013 & A020 & A024 & A050 & A059 & A060 & A089 & A092 & A093 \\
\hline
\end{tabular}

